\title{
Jahrbuch der berufs- und wirtschaftspädagogischen Forschung 2016
}

Jürgen Seifried, Susan Seeber, Birgit Ziegler (Hrsg.)

DGE Deutsche Gesellschaft 
Schriftenreihe der Sektion

Berufs- und Wirtschaftspädagogik der Deutschen Gesellschaft

für Erziehungswissenschaft (DGfE) 
Jürgen Seifried

Susan Seeber

Birgit Ziegler (Hrsg.)

Jahrbuch der berufs- und

wirtschaftspädagogischen

Forschung 2016

Verlag Barbara Budrich

Opladen • Berlin • Toronto 2016 
Bibliografische Information der Deutschen Nationalbibliothek

Die Deutsche Nationalbibliothek verzeichnet diese Publikation in der Deutschen

Nationalbibliografie; detaillierte bibliografische Daten sind im Internet über

http://dnb.d-nb.de abrufbar.

(C) 2016 Dieses Werk ist im Verlag Barbara Budrich erschienen und steht unter folgender Creative Commons Lizenz: http://creativecommons.org/licenses/by-nc$\mathrm{nd} / 3.0 / \mathrm{de} /$

Verbreitung, Speicherung und Vervielfältigung erlaubt, kommerzielle Nutzung und Veränderung nur mit Genehmigung des Verlags Barbara Budrich

Dieses Buch steht im OpenAccess Bereich der Verlagsseite zum kostenlosen Download bereit (http://dx.doi.org/10.3224/84740588)

Eine kostenpflichtige Druckversion (Printing on Demand) kann über den Verlag bezogen werden. Die Seitenzahlen in der Druck- und Onlineversion sind identisch.

\section{ISBN 978-3-8474-0588-7 (Paperback) \\ eISBN 978-3-8474-0403-3 (eBook) \\ DOI $\quad 10.3224 / 84740588$}

Umschlaggestaltung: Bettina Lehfeldt, Kleinmachnow - www.lehfeldtgraphic.de Typographisches Lektorat: Angela Zerfaß, Leverkusen 


\section{Inhaltsverzeichnis}

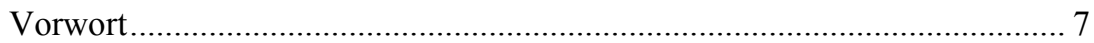

\section{Teil I: Organisation, Bedingungen und Strukturen der beruflichen Bildung}

Allison Fuller

Developing expertise: occupational versus job-based approaches in

contemporary labour markets

Lorenz Lassnigg

„Duale“ oder „,dualistische“ Berufsbildung: Gemeinsamkeiten und

Unterschiede Österreich-Schweiz-Deutschland

Philipp Gonon und Lea Zehnder

Die Berufsbildung der Schweiz als permanenter

Kompromissbildungsprozess

Rolf Dobischat, Andy Schäfer, Christian Schmidt, Manfred Wahle und

Marcel Walter

Berufslaufbahnkonzepte: Instrumente zur Weiterentwicklung des

Berufsbildungssystems?...

Karl-Heinz Gerholz und Ursula Walkenhorst

Gestaltungsfragen zur Akademisierung der beruflichen Bildung am

Beispiel der Gesundheitsfachberufe. 73

\section{Teil II: Berufliche Lehr-Lern- und Unterrichtsforschung}

Juliana Schlicht

Handeln in Geschäftsprozessen als Forschungs- und

Lehr-Lern-Gegenstand

Alexandra Bach

Nutzung von digitalen Medien an berufsbildenden Schulen -

Notwendigkeit, Rahmenbedingungen, Akzeptanz und Wirkungen 107

Stefan Kessler

Branchen- und lernortspezifische Herausforderungen beim Einsatz von

Tablets in der überbetrieblichen Ausbildung der Schweizer Banken 125 


\section{Teil III: Hochschul- und Lehrerbildungsforschung}

Julia K. Gronewold

Struktur und Organisation berufsbegleitender MINT-Studiengänge

Lena Hillebrecht

Entwicklung eines Modells zur Beurteilung von Qualität in

berufsbegleitenden Studiengängen

Christoph Helm und Stefanie Antonia Mayer

Pedagogical Content Knowledge von Rechnungswesenlehrkräften -

Explorative Befunde einer Onlinebefragung aus Österreich

\section{Teil IV: Betriebliche Aus- und Weiterbildung}

Philipp Struck und Christian Dittmann

Weiterbildung im Handwerk. Rahmenbedingungen und

Handlungsfelder aus der Perspektive von Betrieben und Beschäftigten ..... 189

Andreas Rausch, Julia Warwas und Heike Jost

Die Beurteilung kaufmännischer Auszubildender in der betrieblichen

Praxis - Eine explorative Studie bei Industrie- und Bankkaufleuten

\section{Teil V: Historische Berufsbildungsforschung}

Frank Ragutt

Lesbarkeit der Branchen - Notizen zur Ordnungssemantik des Gewerbes in Realienbüchern des 19. Jahrhunderts.................................................... 223

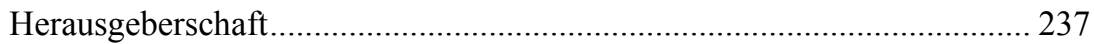

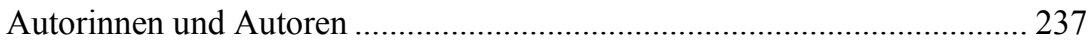




\section{Vorwort}

Mit Blick auf die thematische und methodische Vielfalt der deutschsprachigen Berufsbildungsforschung - diese bearbeitet z.B. Fragen der Didaktik und Methodik des beruflichen Lernens, der Kompetenzmodellierung und Kompetenzmessung, der Lehrerbildungsforschung, der beruflichen Aus- und Weiterbildung, der Hochschuldidaktik sowie der historischen Berufsbildungsforschung - entschloss sich die Sektion für Berufs- und Wirtschaftspädagogik der Deutschen Gesellschaft für Erziehungswissenschaft (DGfE) im Jahr 2012, ein „Jahrbuch der berufs- und wirtschaftspädagogischen Forschung” herauszugeben, um so der Breite und Tiefe der Forschung und Theoriebildung in der Berufs- und Wirtschaftspädagogik gerecht zu werden. Die Aufgabe des Jahrbuchs besteht darin, die im Rahmen der Sektionstagungen geführten Diskussionen zu dokumentieren und damit einen Einblick in den aktuellen Stand der Berufsbildungsforschung zu geben. Mit dem hier vorgelegten vierten Jahrbuch sollen die Arbeiten der Sektion Berufs- und Wirtschaftspädagogik erneut einer interessierten Öffentlichkeit zugänglich gemacht werden.

Die im September 2015 an der Universität Zürich durchgeführte Jahrestagung der Sektion Berufs- und Wirtschaftspädagogik der DGfE erfreute sich einer sehr guten Resonanz. Das aktuelle Jahrbuch präsentiert daher insgesamt 14 Beiträge, die wie gewohnt einem doppelten Reviewverfahren unterzogen wurden.

In einem ersten Abschnitt widmet sich das Jahrbuch ausgewählten Fragestellungen der Organisation, Bedingungen und Strukturen der beruflichen Bildung. Allison Fuller diskutiert in ihrem grundlegenden Beitrag die Frage nach verschiedenen Ansätzen zur Entwicklung von Expertise. Dabei beschäftigt sie sich insbesondere mit der beruflichen Ausbildung. Vor dem Hintergrund der Erfahrungen in England stellt sie die Notwendigkeit der Entwicklung einer umfassenden beruflichen Expertise heraus (z.B. auch mit Blick auf die berufliche Identität) und diskutiert - u.a. pointiert am Beispiel der Ausbildung zum Golf Greenkeeper - die Bedeutung und die Reichweite des Begriffs „Occupation“ (respektive des deutschen Berufsbegriffs) in Abgrenzung zum Konzept „Job“.

Im sich anschließenden Beitrag von Lorenz Lassnigg steht die Frage nach Gemeinsamkeiten oder Unterschieden einer „dualen“ oder „dualistischen" Berufsbildung in Österreich, der Schweiz und Deutschland im Blickpunkt. Diese werden besonders unter der Perspektive der gemeinhin unterstellten positiven Wirkung der in diesen Ländern etablierten Strukturen der Erstausbildung auf die Jugendarbeitslosigkeit analysiert. Die Unterschiede zwischen den Ländern sieht der Autor zum einen historisch begründet und zum anderen im gesellschaftlichen Umfeld verortet. 
Philipp Gonon und Lea Zehnder thematisieren in ihrem Beitrag die Notwendigkeit der Kompromissfindung in der Berufsbildungspolitik und illustrieren den Sachverhalt am Beispiel des Berufsbildungsgesetzes in der Schweiz. Aus einer Steuerungsperspektive, so Gonon und Zehnder, ist Berufsbildung ,in der Regel ein ausgehandelter Kompromiss unterschiedlicher Anliegen“" (S. 43). Dabei tragen insbesondere die Möglichkeiten der Hybridisierung zur Kompromissbildung bei, wobei eine horizontale (z.B. das Zusammentreffen von Persönlichkeitsbildung und beruflicher Qualifizierung) und eine vertikale Hybridisierung (Kombination von Arbeitsmarktbefähigung mit einer $\mathrm{Zu}-$ gangsberechtigung zum Hochschulsystem) zu unterscheiden ist.

Im Beitrag von Rolf Dobischat, Andy Schäfer, Christian Schmidt, Manfred Wahle und Marcel Walter geht es dann um Berufslaufbahnkonzepte als Instrument zur Weiterentwicklung des Berufsbildungssystems. Die Autoren zeigen wesentliche Entstehungsbedingungen auf, die für die Formulierung von Berufslaufbahnkonzepten von Bedeutung sind. Dabei werden vor dem Hintergrund von sich verändernden Qualifikationsanforderungen und steigender beruflicher Mobilität insbesondere Entwicklungen bezüglich der abnehmenden Attraktivität des dualen Systems für leistungsstärkere Jugendliche (bzw. der Trend zur Akademisierung) einerseits und die Herausforderung, leistungsschwächere Jugendliche zu intergrieren andererseits, als relevant erachtet. Es folgen eine Verdichtung von Kernelementen bestehender Laufbahnkonzepte zu einem Laufbahnmodell sowie eine Diskussion der Funktionen von Laufbahnkonzepten.

Karl-Heinz Gerholz und Ursula Walkenhorst schließlich zeigen am Beispiel der Ausbildung in den therapeutischen Gesundheitsfachberufen auf, inwiefern der Trend zur Akademisierung das Verhältnis von Berufsbildung und Hochschulbildung bestimmt. Die Autorin und der Autor arbeiten Problemfelder auf der Makroebene (Beschäftigungssystem, Bildungspolitik und Bildungssystem), Mesoebene (curriculare und organisatorische Umsetzung der Akademisierung resp. Studiengangsgestaltung) sowie Mikroebene (LehrLernprozessgestaltung) heraus.

Im zweiten Abschnitt des vorliegenden Bandes stehen Beiträge zur beruflichen Lehr-Lern- und Unterrichtsforschung im Mittelpunkt.

Juliana Schlicht zeigt exemplarisch mittels der Analyse von Geschäftsprozessen (zur Personalplanung, zur Personalentwicklung, zum Regulierungsmanagement, zur Auftragsbearbeitung sowie zum Kundenzufriedenheitsmanagement) in einem Unternehmen der Energiewirtschaft, wie der Anspruch an eine umfassende Geschäftsprozessorientierung umgesetzt werden kann. Sie betont dabei die Notwendigkeit, ,güter- und leistungsbezogene, finanzielle und informationstechnische Tätigkeitsaspekte stärker als bisher mit sozialen und emotionalen Bedingungsfaktoren des Handelns in Geschäftsprozessen thematisch zu verknüpfen" (S. 92) und verweist auf die Schwierig- 
keit, zwischenmenschliche Kommunikation und Kooperation in Geschäftsprozessmodellen hinreichend gut abzubilden.

Alexandra Bach arbeitet in ihrem Beitrag zur Nutzung von digitalen Medien an berufsbildenden Schulen die Notwendigkeit der Integration von digitalen Medien in den Unterricht an beruflichen und allgemeinbildenden Schulen heraus. Unter Rückgriff auf eine adaptierte Fassung des Technologieakzeptanzmodells (UTAUT: „Unified Theory of Acceptance and Use of Technology“ - hier wird neben einem Zusammenhang zwischen Nutzungsabsicht und tatsächlicher Nutzung postuliert, dass beide Variablen durch Faktoren wie Leistungserwartung, Anstrengungserwartung, sozialer Einfluss und unterstützende Bedingungen beeinflusst werden) berichtet die Autorin empirisch belegte Bedingungsfaktoren der digitalen Medienverwendung. Die empirische Überprüfung des Modells für berufsbildende Schulen steht allerdings noch aus.

Ebenfalls mit digitalen Medien befasst sich die Studie, über die Stefan Kessler berichtet. Ausgangspunkt ist, dass ab August 2012 in der Schweiz ein Großteil der Lernenden in einer kaufmännischen Bankenausbildung zu Ausbildungsbeginn mit Tablets ausgestattet wird, die in überbetrieblichen Kursen zum Einsatz kommen. Es zeigt sich der zunächst überraschende Befund, dass der Einsatz von Tablets seitens der Lernenden auf eine stellenweise nur geringe Akzeptanz stößt. Vielmehr lassen sich Effekte der Entäuschung feststellen (große Vorfreude auf die Tablet-Nutzung bei anschließender Ernüchterung). Als Gründe hierfür werden in erster Linie technische Limitationen des Tablets, die einer effizienten Nutzung während der Ausbildung entgegenstehen, ins Feld geführt. Zudem kommen Tablets offenbar am Arbeitsplatz selbst nur selten zum Einsatz.

Der Schwerpunkt des dritten Abschnitts des Jahrbuches liegt auf Fragen der Hochschul- und Lehrerbildungsforschung. Zunächst berichtet Julia Gronewold über eine Studie zur Analyse der Struktur und Organisation berufsbegleitender MINT-Studiengänge. Auf Basis von Interviews mit 16 Lehrenden und Studiengangskoordinatoren geht sie Forschungsfragen auf der Mikroebene (Herausforderungen für Lehrende und Studiengangskoordinatoren), der Mesoebene (Rolle der Kooperationen mit Unternehmen und Bildungsanbietern) sowie der Makroebene (Analyse der Bildungsstruktur und Bildungsorganisation) nach und arbeitet für jede Ebene Veränderungs- und Weiterentwicklungsbedarfe heraus.

Lena Hillebrecht stellt die Entwicklung eines Modells zur Beurteilung der Qualität von berufsbegleitenden Studiengängen dar, das als AngebotsNutzungs-Modell konzipiert ist und einer empirischen Überprüfung unterzogen wird. In dem Beitrag werden erste Befunde aus einer Pilotstudie berichtet, bei der insgesamt über 100 Studierende, die ein berufsbegleitendes Studium absolvieren, befragt wurden. Es zeigt sich, dass für berufsbegleitend Studierende bei der Beurteilung der Studienqualität insbesondere Studiengangs- 
merkmalen (mit Blick auf die „Machbarkeit des Studiums“) Bedeutung zukommen.

Christoph Helm und Stefanie Antonia Mayer berichten im Anschluss über eine Studie zur Messung des fachdidaktischen Wissens von ca. 90 Rechnungswesen-Lehrkräften in Österreich. Dabei werden in Anlehnung na gängige Kompetenzmodellierungen die fachdidaktischen Wissensfacetten „Wissen über Schülerkognitionen“, „Wissen über das Zugänglichmachen von Inhalten“ sowie „Wissen über das Potenzial von Aufgaben“ operationalisiert. Die Befunde verweisen darauf, dass die Wissensindikatoren lediglich schwach bis moderat zusammenhängen.

Dem sich anschließenden Teil IV (Betriebliche Aus- und Weiterbildung) sind zwei Beiträge zuzurechnen. Philipp Struck und Christian Dittmann stellen die Ergebnisse einer Studie vor, die den Stellenwert beruflicher Weiterbildung für Betriebe und Beschäftigte im Handwerk thematisiert. Auf Basis der Ergebnisse einer Befragung von über 100 Geschäftsführern und Betriebsratsmitgliedern entwickeln die Autoren eine Skala zur Erfassung des Stellenwerts der beruflichen Weiterbildung.

Andreas Rausch, Julia Warwas und Heike Jost präsentieren die Ergebnisse einer explorativen Studie zur Beurteilung kaufmännischer Auszubildender in der betrieblichen Praxis. 136 Ausbildungsbetriebe nahmen an der Studie teil, und es zeigt sich, dass die in den Betrieben eingesetzten Beurteilungsbogen primär auf eine Bewertung allgemeiner Arbeitstugenden und sozialer Kompetenzen abstellen.

Der Beitrag von Frank Ragutt (Abschnitt IV: Historische Berufsbildungsforschung) beschließt das Jahrbuch. Der Autor gibt unter dem Titel „Lesbarkeit der Branchen“ einen profunden Überblick über die historische Schulbuchforschung für die berufliche Bildung.

Der Vorstand der Sektion Berufs- und Wirtschaftspädagogik der DGfE bedankt sich sehr herzlich bei Professor Dr. Franz Eberle und Professor Dr. Philipp Gonon (Universität Zürich) sowie den dortigen Mitarbeiterinnen und Mitarbeitern für die Ausrichtung der Sektionstagung. Unser Dank gilt zudem den Gutachterinnen und Gutachtern, die die eingegangenen Beiträge in gewohnt akribischer Manier unter die Lupe nahmen und viele konstruktive Hinweise zur Verbesserung der Texte lieferten. Schließlich danken wir Frau Sarah Schmauder sowie Frau Carolin Volz für die Mühe bei der Formatierung der Beiträge.

Mannheim, Göttingen und Darmstadt, im Juli 2016

Jürgen Seifried, Susan Seeber und Birgit Ziegler 


\title{
Developing expertise: occupational versus job-based approaches in contemporary labour markets ${ }^{1}$
}

\author{
Alison Fuller
}

\section{Introduction}

The provision of an appropriate supply of occupational expertise is clearly of overwhelming importance in the contemporary labour market. Without it, how would the infrastructure that supports modern life be built, how would the sick and vulnerable be properly cared for; how would machinery and equipment be effectively maintained and repaired, and so on. All these activities are made possible because there are people available who have benefited from vocational education and training (VET), and have acquired the relevant occupational knowledge, skills and expertise that has enabled them to become competent practitioners.

However, there is a long tradition of scholars (such as John Dewey and Georg Kerschensteiner) who, historically, have argued that vocational learning has more than an instrumental function. They have argued for an holistic and humanistic concept of vocational education that recognises the inherent and intrinsic values for the individual, economy, and wider society associated with learning for occupational expertise and in the practice of skilled work. A contemporary scholar, Philipp Gonon, has summed up why this view is still relevant. He signals the continuing value of an approach to providing VET that transcends the requirements of particular employers and individuals, in order to fulfill broader economic and societal functions and interests.

"Vocational education that includes more than simply technical training is a model that successfully balances different interests" (Ganon 2009, 20).

In this chapter, I first consider different conceptions of VET and how these are linked to different approaches to developing occupational expertise. I then focus particularly on apprenticeship, discussing the strong historical relationship between apprenticeship and the concept of occupation. The discussion highlights the contested relevance of the concept in the context of contemporary labour markets by drawing on apprenticeship in England to

1 An earlier version of this paper was presented to the Vocational Education and Workforce Congress, University of Zurich, 8 September 2015. 
highlight some of the weaknesses of a narrowly conceived, competencebased model.

Writing around a hundred years ago, the American philosopher John Dewey highlighted the importance of understanding the symbiotic relationship between the availability and character of vocational education and the economic and industrial context in which it is located (Dewey 2010). His insight is still highly relevant. Contemporary policy makers, employers, VET providers and researchers are all questioning the extent to which vocational education pathways should be expected to fulfill a range of objectives including: the preparation of young people for specific jobs with specific skills or, more broadly for a changing, dynamic and global labour market that requires adaptability for occupational and career changes; the production of active citizens able to participate fully in $21^{\text {st }}$ century democracies; and, the ability to support and facilitate youth transitions from education to work and into adulthood in a period of economic hardship and intense global competition.

The purpose of VET can then be viewed on a spectrum with training for specific, narrowly defined jobs at one end and, at the other, a more general preparation designed to help young people lead productive and fulfilling adult lives as workers and citizens. Each has different implications for the development of occupational expertise, including the extent to which VET programmes are designed to include the teaching and learning of vocational knowledge and the relevant underpinning scientific, technical and social theories, or, instead, are organised in terms of a list of functional competences and standards that participants need to show they have met.

The recent international revival of interest in apprenticeship, and the development of national policies to support the evolution and expansion of programmes is symptomatic of renewed interest in the importance of occupational skill formation and the workplace as a learning environment (Fuller and Unwin 2012). Apprenticeship is a highly resilient concept that can be understood in two key ways. First, as an institution, it is the name for a set of formalised, state-regulated arrangements designed to produce skilled workers. In many European countries, these arrangements are organised through social partnerships between the State, employers, trades unions, and education and training providers; and that are created as programmes of learning and practical workplace training and experience targeted at young people. Second, apprenticeship can be understood metaphorically. In this regard, apprenticeship has meaning for people from a range of occupational fields. It has been used, since medieval times, as a term to describe the learning journey a person takes from novice to expert in a specific craft, trade or occupation. The term transcends occupational boundaries and hierarchies, and is used by surgeons as well as carpenters, chefs, actors and musicians to indicate how at they have developed their occupational expertise through practice over time, and that this has enabled them to reach the point where they can 
work without supervision and be accepted as a full member of an occupational community. Lorna Unwin and I (Fuller \& Unwin 2008, 12) have argued that "whilst the culture of workplaces and the nature of the relationship between young people and adults evolve, the broad conception of learning as becoming part of an occupational community remains at the heart of the apprenticeship model".

Apprenticeship as a model of learning for occupational expertise and membership implies becoming a different person. To ignore this aspect of learning is to overlook the point made so cogently by Jean Lave and Etienne Wenger (1991), that learning involves the construction of identities, with knowing and membership of a community of practice entailing one another. Lave and Wenger's concept of 'communities of practice' has been criticised for promoting an overly conservative picture of the reproduction of the same types of bounded expertise (what the medieval guilds referred to as their 'secrets') and expected behaviour from one generation of employees to the next within settings that remain stable. Critics have argued that the contemporary workplace is a much more unstable, dynamic and contested space (Hughes et al. 2007). Yet, Lave and Wenger's concept has value because it draws attention to the symbiotic relationship between individuals and the development of their work and occupational identities, and the social relations and conditions in which this is nurtured.

\section{Changing relationship between apprenticeship and occupation}

Just as critics have argued that contemporary workplace communities of practice are dynamic and contested, there is a parallel argument that problematizes the ongoing stability and relevance of the concept of occupation. This has implications for apprenticeship, which I now want to explore through referring to the English case. ${ }^{2}$ Historically, in England, apprenticeship was strongly aligned with the concept of skilled occupations. However in the late 1980s, competence-based qualifications were introduced in England. These were based on 'standards' that were maintained by the sectoral bodies. Since then the concept of sector has come to be used as a supply-side mechanism for the organisation of government-funded VET. In keeping with this shift, the introduction of government supported apprenticeship in the early to mid-1990s was based on a sectoral competence-based, rather than an

2 A longer version of this discussion can be found in Fuller, A. and Unwin, L. Apprenticeship and the Concept of Occupation, London: Gatsby Charitable Foundation. 
occupational, model. This is expressed through the creation of apprenticeship as a 'wrapper' for a set of task-related competences located and assessed within sector frameworks, rather than being seen as a programme of learning leading to a recognisable occupational identity with clear labour market currency (Fuller \& Unwin 2013).

Whilst in some English apprenticeships, for example in areas of engineering and construction, hairdressing, and parts of hospitality, the relationship of apprenticeship to occupations still has meaning due to the maintenance of a strong and long-standing apprenticeship culture, in others, for example, customer service, parts of retail, business administration, health and social care the connection is underdeveloped and even non-existent. This contrasts with the way apprenticeship is defined, designated and conceptualised in some other European countries and North America, and to some extent in Australia.

A useful way to approach the meaning of occupation is to consider the term 'job' which, in the UK, is sometimes used interchangeably with occupation. Linda Clarke (2011) has argued that the term 'job' has a much more limited meaning than occupation because it is connected to an employment contract in a workplace. Hence, a job description lists the tasks an individual is required to perform. In contrast, an 'occupation' is a 'much more general and all-encompassing term for employment' in which individuals are engaged and is not restricted to a particular employer or workplace. From Clarke's perspective, 'occupation' is aligned with the German concept of Beruf which is equated to the idea of vocation and is applicable across the socially constructed hierarchies of more and less prestigious occupations or professions.

In his classic text about the 'labour process under capitalism' published in the early 1970s, Harry Braverman $(1974,109)$ argued that up until Frederick Winslow Taylor developed his scientific management techniques in the late 19th century, 'the craft or skilled trade was the basic unit, the elementary cell of the labour process.' For Braverman, occupations became deskilled or, in the case of crafts, wiped out as a result of Taylor's efficiency revolution and the removal of the opportunity for workers to conceptualise and have discretion over their own work tasks. Like Braverman, but writing much more recently, Richard Sennett (2008) has argued that Tayloristic or Fordist forms of labour process severely restrict opportunities for people to develop and deploy their occupational expertise.

The concept of occupation is central, then, to understandings of how labour markets are organised as well as to the interlinked development of identity and expertise, occupational identity should not be seen as a static concept, but one that is dynamic and multi-faceted. Individuals construct and have their occupational identities shaped within a) changing institutional and 
cultural contexts; b) the social relations of particular workplace learning environments, and c) changing labour market conditions and hierarchies.

The development of an occupational identity (the process of 'becoming') takes time and commitment and, hence, the process of maturation has always been seen as central to apprenticeship. In his discussion of the development of occupational self-concept and commitment to an occupation by apprentices in printing, R.A. Flude (1977) talked about the concept of 'anticipatory socialisation' - the notion that as an individual progresses through their training, they become more likely to identify themselves as a full member of their occupational group. Back in the 1970s, Flude was arguing that apprentices were not just training for a job role, but to have the status of full members of an occupational community. However, as Braverman's analysis has highlighted, industrial and technological innovation in the post-second world war era undermined the demand for and availability of traditional occupations and skilled trades.

By the 1990s writers such as Robert Reich ${ }^{3}$ (1991) and Catherine Casey (1995) were conceiving advanced industrial liberal economies such as the UK and the US as post-occupational. The implications of this for occupational identity and the development of occupational expertise, as well as for the character of labour markets, were significant. Expectations for many people were shifting away from the notion of training for life-long employment in a secure and stable occupation to a recognition, either that it would be necessary to move between jobs as their original skills became obsolete, or that the range of skills required to achieve occupational expertise would have to alter. Reich argued that in the context of rapid industrial change and globalisation, a radical re-categorisation of work was needed, which did not depend on defined occupations as its basis.

Building on his analysis of the US economy, Reich (1991) argued that three over-arching work roles were emerging. They were 'routine production services', 'in-person services' and 'symbolic-analytic services' which offered broad concepts of work activity that were decoupled from the notion of specific occupation. Routine production services referred to jobs in mass production enterprises, whilst in-person services referred to routine interpersonal or customer-facing roles. The final category, 'symbolic analytic services', referred to 'problem-solving, problem identifying, and strategic brokering activities' and as including all those who 'solve, identify and broker problems by manipulating symbols'. According to Reich it was only the last category, the symbolic analysts, comprising about 20 per cent of the American workforce in the early 1990s, which he saw as having an increasingly strong position in contemporary international labour markets based on their ability to add value to an enterprise through the creative use of knowledge, skills and

3 Robert Reich was Secretary of Labor under President Bill Clinton from 1993 to 1997. 
experience. In contrast with the past, increasing the number of 'symbolic analysts' would require the expansion of higher education (rather than intermediate vocational education, including apprenticeship) as the vehicle for creating the level of attainment and generic skills that could enable the workforces in countries like the US to compete in the new order of the 'global knowledge economy'.

However, whilst it is true that, in recent years, higher education has expanded significantly in the United States and many other countries this has not always been coupled with a diminution of interest in VET and the development of intermediate skills. In particular, and as I have highlighted, there has been a revival of international interest in growing the apprenticeship route. I think it is important, then, to recognise the relationship between industrial and technological change, the nature of occupational expertise, and the structure and character of labour markets, and how these vary across countries with different institutional and cultural VET histories.

For example, in Germany the holistic notion of Beruf continues to underpin its VET system and, crucially, this is supported through regulated occupational labour markets. In the German context, the development of occupational identity and expertise continues to be reinforced through standardised apprenticeship training ordinances as well as by the legal definition and protections of skilled occupations. This regulated and standardised institutional model of apprenticeship promotes a collective approach to skill formation, a commitment to occupation as distinct from, although it can be in addition to, the commitment an individual may feel to their employing organisation. It also plays an important role in developing citizenship. Despite the ongoing strengths of this approach, questions are asked about the ability of the model to adapt in response to industrial, commercial and technological change, and competition from across the global economy and labour markets. With this backdrop in mind, young people and their families have to consider what the best education and training strategy may be for securing future labour market position and well-being. Whilst the dual system remains strong in countries such as Germany, interest in participation in higher education has increased, with new progression routes opening up (Deissinger, Aff, Fuller \& Jorgensen 2013).

In countries such as England that have labour markets which are more flexible, the institutional supports and arrangements for the development of occupational expertise, identity and commitment are quite weak. Importantly, there is limited occupational regulation or use of licence to practise provisions and this restricts the benefits (eg wage premium) that can accrue to the completion of an apprenticeship. Individuals are increasingly expected to change jobs throughout their working lives, with career progression tending to be linked to internal, rather than external occupational, labour market arrangements. This produces a model of commitment that privileges the rela- 
tionship between individual and employer rather than between individual and the occupation, and militates against collective institutional approaches to skill formation.

Earlier in this chapter, I described apprenticeship as a model of learning for occupational expertise which takes the apprentice on a journey to becoming a full member of an occupational community. This assumes, firstly, that there is a defined occupational community to join; and, secondly, that apprenticeship is a recognised and formalised route to achieving the relevant occupational expertise required to join the community. What follows from this is that each occupation has a defined knowledge-base and associated curriculum which has to be completed and examined in order for the apprentice to show that they meet the requirements to practise as a recognised member of the community. As a result, the apprentice has, at the outset, a clear sense of the occupation they are aiming for. They know that if they meet the requirements they will gain the necessary certification for employment in that occupation.

However, the government-supported apprenticeship programme in England has not been underpinned by a strong model of learning and concept of occupation. Instead, it is underpinned by the perceived needs of employers in a highly flexible labour market in which typically people can be easily switched between job roles within very broadly defined sectoral categories. In addition, manufacturing and engineering, areas strongly associated with skilled technical work, well-defined education and training pathways and a strong concept of occupation, currently only accounts for about 10 per cent of the UK economy. Only about 3 per cent of those becoming an apprentice in England are starting an engineering apprenticeship.

\section{Apprenticeship as a job rather than an occupation: the English case}

Government supported apprenticeship in England is conceived first and foremost as a job. This is in stark contrast to an occupationally-based approach, which has at its heart the concept of participation in a staged 'journey' towards recognised occupational expertise. An important illustration of the difference is that apprenticeships in England are offered at different qualification and skill levels ranging from semi-skilled to bachelor degree level. This fragmented approach sets it apart from other countries where apprenticeships are only available for occupations which have been formally recognised as 'skilled', and where there is one integrated apprenticeship per defined occupation. The most recent statistics for 2014-15 (House of Commons Library, January 2016) indicate that 60 per cent of apprenticeships undertak- 
en in England are only at Level 2 - the semi-skilled level and are linked to job titles such as 'warehouse assistant', shop assistant, and 'food production'.

The English system implies that novices have to complete more than one apprenticeship to climb the relevant ladder of occupational expertise, but there is no guarantee that the next level of apprenticeship will be available. This means that people can get 'stuck' and fail to complete their journey. So, if say a 17 year old pursues a Level 2 (semi-skilled) apprenticeship usually completing in about 12 months, they may not be able to progress to a Level 3 apprenticeship and attain skilled status. Their progression will depend on whether their employer can offer them a job which requires Level 3 skills. Without such a job being available, they will not be able to produce evidence that they have undertaken, and been assessed as competent in, the relevant tasks. Moreover, on the basis of their Level 2 apprenticeship alone, they are less likely to have developed the necessary general education attainment to enable them to progress easily in their educational career either. Whilst, apprentices are required to pursue English and mathematics as part of their apprenticeship, they only have to study the subjects at a level below the apprenticeship itself. For example, those completing a Level 2 programme are required to attain English and mathematics at Level 1 and pursue (but not qualify in) the subjects at Level 2 during the course of their apprenticeship.

The fragmented and narrow approach to apprenticeship is a weakness in the English model. However, this does not mean that there are no high quality apprenticeships in England. Where the scheme has been grafted on to an occupation with a strong training history and culture, for example in some forms of engineering, there are examples of superb practice and what Lorna Unwin and I have called 'expansive apprenticeships' (2003). However, the overall picture is one of a weak relationship between occupation and apprenticeship and a minimalist approach to the inclusion of general education.

There are currently nearly 200 apprenticeship frameworks in England covering around 1,700 job titles and job roles. The five most highly populated apprenticeship sector frameworks are Health and Social Care, Business Administration, Management, Hospitality and Catering, and Customer Service - all in service areas. The minimum length for a government-funded apprenticeship is 12 months. In response to criticisms about the quality of apprenticeship in England, the Conservative government has initiated a further process of reform. This involves small groups of employers (known as 'Trailblazers') coming together to devise and determine a single standard for each apprenticeship. ${ }^{4}$ Approved trailblazer standards become eligible for government funding.

A focus on one of the new apprenticeship standards helps to show that the English reforms do not involve moving the model away from a job-based to

4 Further information about this approach is available at https://www.gov.uk/government/collections/apprenticeship-standards. 
an occupationally-based approach. To illustrate this, we can look at one of the Trailblazer standards that has recently been given approval for public funding and delivery as an apprenticeship: as a 'Golf Greenkeeper'. The description of the role states:

A Golf Greenkeeper is responsible for the maintenance, care and overall appearance of a golf course in line with employer's requirements. It is their job to maintain a good quality playing surface and ensure the course offers a consistent challenge and an enjoyable experience for golfers. ${ }^{5}$

The standard is pitched at Level 2 - positioning this job at a semi-skilled level with apprentices only required to achieve Level $1^{6}$ in English and Mathematics (the general education component of the standard). This means that even those successfully completing the apprenticeship would only have achieved a level in English and Mathematics expected of 14 year olds. Another interesting issue to note is the degree of specialisation associated with this standard, which illustrates that this apprenticeship is not underpinned by a strong concept of occupation. A more holistic conception would have focused on the development of knowledge and skills associated with becoming a broader based expert, qualified to maintain grounds across a range of sports and leisure activities including say football, rugby, cricket, bowls, as well as golf. Limiting the role to golf green keeping signals a level of specificity likely to generate a narrow occupational identity, restrict the apprentice's access to vocational knowledge concepts and principles relevant to understanding the properties of diverse natural and artificial playing surfaces and types of ground; limit the apprentice's subsequent employment opportunities, and opportunities for educational progression.

The fragmented and segmented character of the English model is retained under the Trailblazer reforms. As the example of the golf greenkeeper illustrates there is no concept of an integrated journey of skill formation leading to recognition as a skilled practitioner in a broad occupational field. Rather than having one standard covering the whole trajectory from novice to expert, the individual must navigate their way along a difficult and obscure path. The completion of the Level 2 Golf green keeper apprenticeship only takes them part of the way on that journey and, although a Level 3 apprenticeship exists, they are not guaranteed access to this next rung of the skill ladder - known as 'Advanced Golf Greenkeeper' apprenticeship:

The apprenticeship is designed to provide the greenkeeper with the skills and knowledge to operate at an advanced level where supervisory and/or

5 See https://www.gov.uk/government/uploads/system/uploads/attachment_data/file/372241/ GOLF_GREENKEEPING_HORTICULTURE_Golf_Greenkeeper.ashx.pdf accessed 29 January 2016.

6 Level 1 is the standard expected by age 14, with Level 2 by age 16 . 
specialist technical and agronomic skills are required. Upon completion the advanced greenkeeper will have the necessary skills to supervise the greenkeeping team and the maintenance of the golf course, in the absence of the Head Greenkeeper/Golf Course Manager, and will assist in the planning, supervising and implementing the maintenance, care and overall appearance of a golf course in line with the employer's requirements. ${ }^{7}$

The advanced standard outlined above is highlighted in terms of two dimensions, 'technical agronomic skills' and supervisory and management responsibilities. Completion of this standard is associated with skilled level work, with the individual being able to practise autonomously as well as overseeing the work of others in the team. However, it is still narrowly defined in terms of 'golf greenkeeping' and associated jobs ('Senior Greenkeeper, First Assistant Golf Greenkeeper, Deputy Golf Course Manager') rather than in more holistic occupational terms. From the perspective of general educational attainment the standard is associated with the achievement of Level 2 in English and Mathematics (the standard normally expected of 16 year olds).

The example of the two apprenticeships in golf greenkeeping illustrate some of the key issues associated with the government's approach to apprenticeship, which set it apart from the well-known dual system model operated (with some variation) in countries such as Switzerland, Germany and Denmark. I draw out three critical messages from the English case. First and fundamentally, the relationship between apprenticeship and occupation is weak; the notion that apprenticeship is a job consisting of task-based competences is the structuring principle. Second, the model does not position apprenticeship as a vehicle for developing a skilled (defined as a minimum as Level 3) level of occupational expertise. Most English apprenticeships are associated with semi-skilled jobs and are available at Level 2. Third, and this is a longstanding criticism of English VET, apprenticeship incorporates a very restricted general education component. It is limited both in terms of educational level (Level 1 or 2) and breadth (only English and Mathematics). This means that apprenticeship generally does not provide young people with a strong platform for educational progression. Even in the advanced standard outlined above, the apprentice is not required to go beyond the level they may have achieved at age 16 (i.e. that they may already have on entry to, or soon after entering the apprenticeship). Whilst it is important to remember and stress that there are examples of extremely high quality apprenticeship available in England, the shortcomings in the system allow weak provision to continue.

7 See https://www.gov.uk/government/uploads/system/uploads/attachment_data/file/455065/ GOLF_GREENKEEPING_Advanced_Golf_Greenkeeper.pdf accessed 29 January 2016. 


\section{Conclusion}

I began this chapter by contrasting a far-reaching, holistic conception of vocational education with a narrow occupationally specific alternative, indicating the range of purposes it may be required to fulfill. The ensuing analysis has indicated some of the important dimensions along which approaches to VET and apprenticeship can be differentiated and some of the implications for social justice and inclusion, as well as for educational and career progression.

Case study research is important here as it provides an effective way of identifying and explaining the diverse characteristics of different approaches to VET. Evidence can be generated that shows, on the one hand, how approaches to VET reflect deep-seated historical, cultural, economic, and institutional differences between societies and, on the other, how external pressures and economic forces (e.g. related to the negative economic effects of the banking crisis, changing industrial structure and patterns of international labour mobility, and technological innovation) provide a transformed context with which all industrialized countries are grappling.

Young people are particularly vulnerable to unemployment and to experiencing barriers to their successful transition to fulfilled adult lives and citizenship. The extant literature suggests that the availability of good quality vocational education and apprenticeships can facilitate transitions, but contextual factors mean that what counts as effective, for whom, and in whose interests, is likely to continue to differ.

Finally, I have argued that there are key issues for VET to address in support of developing occupational expertise. In particular I have drawn attention to issues of and tensions between occupational stability versus occupational change; holistic versus narrower approaches to VET and the opportunity to progress life-chances that VET can provide for young people.

\section{References}

Braverman, H. (1974). Labor and Monopoly Capital. New York: Monthly Review Press.

Casey, C. (1995). Work, Self and Society: After industrialism. London: Routledge.

Clarke, L. (2011). Trade? Job? Or Occupation? The Development of Occupationa Labour Markets for Bricklaying and Lorry Driving. In M. Brockmann, L. Clarke \& C. Winch, with G. Hanf, P. Mehaut \& A. Westerhuis, Knowledge, Skills andCompetence in the European Labour Market, (p. 102-119). London: Routledge. 
Deissinger, T., Aff, J., Fuller, A. \& Jorgensen, C. H. (2013). Hybrid qualifications: structures and problems in the context of european vet policy, Studies in vocational and continuing education (vol. 10). Zurich: Peter Lang.

Dewey, J. (2010). Democracy and Education: An introduction to the philosophy of education. Los Angeles: IndoEuropean publishing (revised version of 1923 publication published by New Yor: MacMillan).

Flude, R.A. (1977). The development of an occupational self-concept and commitment to an occupation in a group of skilled manual workers. The Sociological Review, 25(1), 41-49.

Fuller, A. \& Unwin, L. (2008). Towards Expansive Apprenticeship. London: TLRP

Fuller, A. \& Unwin, L. (Eds.). (2012). Contemporary Apprenticeship: International Perspectives on an evolving model of learning. London: Routledge.

Fuller, A. \& Unwin, L. (2013). Apprenticeship and the concept of occupation. London: The Gatsby Charitable Foundation.

Hughes, J., Jewson, N. \& Unwin, L. (2007) (Eds.). Communities of Practice: Critical perspectives. London: Routledge.

Lave, J. \& Wenger, E. (1991). Situated Learning: learning as legitimate peripheral participation. Cambridge: Cambridge University Press.

Reich, R. (1991). The Work of Nations. London: Simon \& Schuster

Sennett, R. (2008). The Craftsman. London: Penguin. 


\title{
„Duale“ oder „,dualistische“ Berufsbildung: Gemeinsamkeiten und Unterschiede Österreich- Schweiz-Deutschland
}

\author{
Lorenz Lassnigg
}

\section{Einleitung}

Aktuell besteht großes und steigendes internationales Interesse an Formen der Lehrlingsausbildung, oder neuerdings collective skills systems (vgl. Busemeyer \& Trampusch 2011), wobei in den politischen Diskursen insbesondere Deutschland aufgrund seiner Größe, aber auch der (deutschsprachigen) Schweiz und Österreich aufgrund der starken Ausprägung dieser Form der Berufsbildung besondere Aufmerksamkeit zukommt. ${ }^{1}$ Neben den Gemeinsamkeiten dieser Länder mit ihrem hohen Anteil an Jugendlichen in organisierten betrieblichen Ausbildungsformen werden aber meist die wesentlichen Unterschiede zwischen den drei Systemen vernachlässigt, auf die sich dieser Beitrag besonders konzentriert. Diese Unterschiede betreffen sowohl die Geschichte und das breitere gesellschaftliche Umfeld (Wirtschaftsstruktur, industrielle Beziehungen etc.), als auch die betrieblichen Praktiken (z.B. unterschiedliche Kosten-Ertragsrelationen), den professionellen Überbau (mit unterschiedlichen Organisationsformen der beruflichen Strukturen und Interessenvertretungen) und schließlich das die Berufsbildung umgebende Bildungswesen in seinen Angebots- und Governancestrukturen, und der Einbindung der Lehrlingsausbildung in die Bildungskarrieren (Übergang in Fachhochschulen, Anbindung an die vorhergehende Pflichtschule etc.).

Der wichtigste Indikator für das große Interesse ist die geringe Jugendarbeitslosigkeit und die damit verbundenen vergleichsweise besseren Übergangsmöglichkeiten für Jugendliche in Beschäftigung, die ansonsten weltweit mit großen Problemen verbunden sind und auch als wichtiges politisches Problem gesehen werden. Hinter diesen groben Bildern im politischen Diskurs besteht jedoch in der Fachwelt wenig Klarheit und Einverständnis darüber, was die wesentlichen Elemente und Wirkungsfaktoren dieser AusBildungsform und Aus-Bildungspraxis sind, und insbesondere darüber, über

1 In diesem Beitrag wird in Anlehnung an den englischen Begriff , apprenticeship“ der neutralere Ausdruck ,Lehrlingsausbildung ' anstelle des deutschen ,Dualen Systems' verwendet, der bereits in den politischen Diskursen stark symbolisch aufgeladen ist (vgl. Lassnigg 2015). 
welche Mechanismen und Praktiken die (relative) Verringerung der Jugendarbeitslosigkeit entsteht (wenn sie entsteht). Auch wird in jüngerer Zeit intensiver diskutiert, welche Voraussetzungen und Möglichkeiten für die Übertragbarkeit (den Transfer oder Export) in Betracht gezogen werden müssen und welche Rolle die pädagogischen Praktiken und die Gestaltung der Lernprozesse in diesem System spielen (wenn es überhaupt ein „System“ ist). Zu diesen Aspekten werden einige Überlegungen und Auswertungen gegen den Strich präsentiert.

Als entscheidendes Argument für die Vorschläge der weltweiten Verbreitung der Lehrlingsausbildung nach deutschem Vorbild wird die geringere Relation der Jugendarbeitslosigkeit im Vergleich zur Gesamtarbeitslosigkeit in Deutschland gegenüber allen anderen G-20-Ländern, wo die Jugendarbeitslosigkeit mindestens das Zwei-Fache bis zum Vier-Fachen der Gesamtarbeitslosigkeit ausmacht, als stylized fact herangezogen (vgl. OECD 2012a, fig. 2, 2). ${ }^{2}$ Entsprechende systematische Ansätze zur Verbreitung der Lehrlingsausbildung gibt es auch auf EU-Ebene ${ }^{3}$ und in Deutschland. ${ }^{4}$

Wichtige Elemente des Diskurses betreffen das Verständnis der Dualität und der Ausprägung des systemischen Zusammenhanges zwischen den Elementen: erstens die unterschiedliche Gewichtung der beiden Pole der Dualität von betrieblicher und schulischer Ausbildung im Zusammenspiel bzw. der Überschneidung von zwei gesellschaftlichen Sphären (Arbeit/Beschäftigung und Lernen/Bildung) mit sehr unterschiedlichen Zwecken, FunktionsLogiken, Praktiken etc. Im Verständnis der Lehrlingsausbildung spielen einerseits die Dimensionen der employment relation (Arbeitsvertrag und Arbeitsrecht) andererseits des ,work-based learning (Lern- und Betreuungsarrangements, Methodik der Unterweisung) eine wichtige Rolle (vgl. Fuller \& Unwin 2013). Hinsichtlich der Systemausprägung ist ein interessanter Wandel von einer definitiv holistisch-systemischen Interpretation des Dualen

2 Vgl. die ausführlichen Informationen dazu auf den OECD-Seiten http://www.oecd.org/ employment/quality-apprenticeships-youth-conference.htm sowie http://www.oecd.org/ employment/action-plan-youth.htm (14.2.2016).

3 Vgl. die European Alliance for Apprenticeships (http://ec.europa.eu/social/main. jsp?catId= 1147\&langId=en) sowie die Aktivitäten im Rahmen von CEDEFOP (http://www.cedefop. europa.eu/en/events-and-projects/projects/apprenticeships-work-based-learning; http://www. cedefop.europa.eu/events/EuropeanApprenticeship2014/) (14.2.2016).

4 Vgl. https://www.bmbf.de/en/the-german-vocational-training-system-2129.html (14.2.2016), sowie Beiträge in den verschiedenen führenden Medien wie Financial Times (http://www.ft. com/intl/cms/s/0/b9008b70-68cf-11e3-bb3e-00144feabdc0.html\# axzz408ZYfuWo), BBC (http://www.bbc.com/news/business-16159943), Wall Street Journal (http://www.wsj.com/ articles/SB10001424052702303665904577452521454725242), Economist (http://www. economist.com/news/europe/21578656-germanys-vaunted-dual-education-system-its-latestexport-hit-ein-neuer-deal) (alles 14.2.2016). 
Systems in der Definition bis in die 2000er Jahre (vgl. Greinert 2005), die das System expressis Verbis unübertragbar macht, zu einer sehr losen pragmatisch-voluntaristischen Aufzählung von Elementen, die je nach Interesse auszuwählen sind, zu beobachten (z.B. die Beiträge in Maurer \& Gonon 2014). In den neueren Europäischen Studien wird das Beschäftigungsverhältnis (Lehrvertrag), das in den ILO-Definitionen noch ein Kernbestandteil war (vgl. Lassnigg 2015), nicht mehr als Element einbezogen, es wird ganz allgemein von irgend einer Form betrieblicher Praxis neben der Schule ausgegangen (z.B. EC 2012). ${ }^{5}$

Busemeyer und Trampusch (2011) konzentrieren sich auf die Unterschiede zwischen collective skills systems und akzentuieren im Vergleich sehr unterschiedliche Aspekte: Für Deutschland wird die Tendenz zum Segmentalismus betont, in dem sich - analog zu Japan - die großen Betriebe auf ihre internen Märkte orientieren und der öffentliche Teil an Bedeutung verliert bzw. zurückgedrängt wird; für die Schweiz wird der Differenzierung auf unterschiedliche levels (Berufsattest, Fähigkeitszeugnis, höhere Berufsbildung) größtes Augenmerk geschenkt; schließlich wird für Österreich die Entwicklung des Dualismus zwischen staatlichen beruflichen Vollzeitschulen einerseits und der Lehrlingsausbildung andererseits herausgearbeitet.

Die Gemeinsamkeiten und Unterschiede der drei Länder im Bereich der Berufsbildung werden im vorliegenden Beitrag ausgehend von tiefergehenden Analysen Österreichs im Hinblick auf die folgenden Aspekte untersucht: Erstens den Zusammenhang zwischen Jugendarbeitslosigkeit und Lehrlingsausbildung (Abschnitt 2), zweitens die Konfigurationen der verschiedenen Elemente der Lehrlingsausbildung (handelt es sich um ein System oder ein Konglomerat?, Abschnitt 3). Drittens wird - ausgehend von der employment relation - der Zusammenhang von Arbeitsmarktpolitik und Arbeitsmarktentwicklung zur Lehrlingsausbildung betrachtet (Abschnitt 4). ${ }^{6}$

5 „This definition makes no explicit reference to the existence of a contractual direct relationship between the employer and the apprentice" (vgl. EC 2012, 11).

6 Aus Platzgründen werden die quantitativen Darstellungen vorwiegend narrativ diskutiert, die quantitativen Informationen sind aus der Präsentation zum Vortrag im Internet (http://www.equi.at/dateien/zuri-keyn-15-DGfE-pdf.pdf) und einem ausführlichen Papier (vgl. Lassnigg 2016; http://www.ihs.ac.at/fileadmin/public/soziologie/pdf/rs114.pdf) zu entnehmen. 


\section{Jugendarbeitslosigkeit und Lehrlingsausbildung - (k)ein klarer Zusammenhang?}

Auf der politischen Ebene wird postuliert, dass eine etablierte Lehrlingsausbildung zur Reduzierung der Jugendarbeitslosigkeit beiträgt. Dieser Zusammenhang wird jedoch nicht genügend differenziert betrachtet. Erstens wird der gängige Indikator für die Jugendarbeitslosigkeit, die Arbeitslosenrate als Anteil der Arbeitslosen am Arbeitskräftepotential oft fälschlich als Anteil der arbeitslosen Jugendlichen an der jugendlichen Bevölkerung interpretiert. Das Arbeitskräftepotential ist jedoch nur der Teil der Jugendlichen, der mehr oder weniger uneingeschränkt dem Arbeitsmarkt zur Verfügung steht. Vor allem aufgrund der steigenden Bildungsbeteiligung (und auch aus anderen Gründen, z.B. einem Rückzug von der Arbeitssuche aufgrund von schlechten Chancen, oft bei jungen Frauen) ist das Arbeitskräftepotential in den meisten Ländern bedeutend kleiner als eine Alterskohorte, was die Arbeitslosenrate statistisch bedeutend über den Anteil der arbeitslosen Jugendlichen an der Bevölkerung erhöht. In Europa kann man davon ausgehen, dass die Arbeitslosenrate unter den Jugendlichen gegenüber dem Anteil der Arbeitslosen an der gleichaltrigen Bevölkerung etwa doppelt so hoch ist (das gilt auch für die oft genannten Länder wie Spanien und Griechenland, wo die oft zitierte Arbeitslosenrate von 50\% einem tatsächlichen Anteil an arbeitslosen Jugendlichen an der jugendlichen Bevölkerung von 15 bis $20 \%$ entspricht, was unakzeptabel hoch genug gewertet werden kann; im EU-Durchschnitt liegen die beiden Werte bei $20 \%$ AL-Rate gegenüber einem 10\%-Anteil, in den drei hier näher betrachteten Ländern liegt dieses Verhältnis bei unter $10 \%$ gegenüber unter 5\%; vgl. Lassnigg 2015; 2016).

Zur Differenzierung des Zusammenhanges zwischen der Lehrlingsausbildung und dem Jugendarbeitsmarkt sind zwei Anmerkungen zu machen: Erstens ist dieser Zusammenhang in einem bestimmten Sinn trivial, indem sich bei einer Gestaltung der Lehrlingsausbildung im Sinne der employment relation (wenn also mit dem Lehr-Lern-Verhältnis eine Arbeitsbeziehung in Unternehmen verbunden ist) die Wahrscheinlichkeit einer nachfolgenden Beschäftigung gegenüber einem Kontrafaktum einer völligen Trennung von Schulbildung und Beschäftigung gleichsam automatisch erhöht (es ist also zunächst v.a. der Kontakt mit dem Unternehmenssektor, nicht unmittelbar die Ausbildungsform, die die Übergangswahrscheinlichkeit erhöht). ${ }^{7}$ Zweitens ist aber (wie bereits bei der Diskussion der Definitionen deutlich wurde) aufgrund unterschiedlicher Gestaltungsformen und Verständnisse von Lehr-

7 Bei der empirischen Analyse der Beschäftigungseffekte lassen sich diese Komponenten schwer trennen, vgl. die Reviews und Analysen in Ecorys, IES \& IRS (2013), wo auch starke Einschränkungen der positiven Wirkungen berichtet werden (vgl. ebd.10-11). 
lingsausbildung in verschiedenen Ländern ein klarer empirischer Zusammenhang bereits in einer sehr einfachen statistischen Betrachtung nicht gegeben (vgl. Lassnigg 2015; 2016). Beobachtet man in der Definition von Hilary Steedman (2012) die Positionierung von Ländern mit Lehrlingsausbildung in der Rangreihe der Länder nach der Höhe der Jugendarbeitslosigkeit, so liegen Deutschland, Schweiz und Österreich zwar am unteren Ende, die acht klassifizierten Länder mit regulierter Lehrlingsausbildung und zwischen 10 und 44 Lehrlingen pro 1.000 Beschäftigten verteilen sich jedoch über das gesamte Spektrum der Jugendarbeitslosigkeit (gemessen am Anteil): Unter den zehn Ländern mit der höchsten Jugendarbeitslosigkeit befinden sich zwei Länder mit Lehrlingsausbildung (Italien und Irland), unter den zehn Ländern mit geringster Jugendarbeitslosigkeit liegen nur vier (die drei Vergleichsländer und Dänemark) mit Lehrlingsausbildung, zwei liegen im Mittelfeld (UK und Frankreich). ${ }^{8}$

\section{Elemente der Lehrlingsausbildung: System oder Konglomerat?}

Insofern also die Lehrlingsausbildung zur Verbesserung des Jugendarbeitsmarktes beiträgt sind nähere Bestimmungen der Elemente und Wirkfaktoren erforderlich, die die niedrige Jugendarbeitslosigkeit als eine Gemeinsamkeit der drei Länder im Unterschied auch zu anderen Lehrlingssystemen beeinflussen. Diese Faktoren können in der Gestaltung der Ausbildung liegen, oder aber außerhalb, in weiteren wirtschaftlichen oder gesellschaftlichen Faktoren (siehe Abschnitt 4).

\subsection{Gestaltungsmerkmale der Lehrlingsausbildung}

Aufgrund der Komplexität der vorhandenen Modelle und Konzeptionen würde eine ausführliche Diskussion hier den Rahmen sprengen (Lassnigg 2015 und 2016 gibt eine ausführliche Darstellung unterschiedlicher Definitionen und Gestaltungsformen, vgl. auch Mazenod 2015; 2016). Die Komplexität

8 „While a positive relationship between apprenticeship and low youth un-employment can be observed over time, it would be misguided to see apprenticeship primarily as a 'cure' for high youth unemployment. [...] it is not a sufficient solution to improving the labour market transition of young people with poor school achievements or other disadvantages." (Steed$\operatorname{man} 2012,2)$. 
der Gestaltungsformen wird an drei Beispielen aus dem rezenten Diskurs illustriert (siehe Tabelle 1).

Diese Konzeptionen sind nicht modular aufgebaut, sondern umfassen die Merkmale, die ein funktionierendes System erfordert, die auf folgenden Dimensionen angesiedelt sind: Balancierung von Bildung/Lernen und Arbeit, sowie von Betrieb und Schule/Bildungszentren; explizite öffentliche Regulation (Governance) und Vertragsgestaltung im Zusammenspiel der verschiedenen Akteure (staatliche Institutionen-Gesetzgebung, UnternehmenBetriebe, Lehrlinge, Berufsorganisationen, Interessenvertretungen etc.); Ausbildungspläne und curriculare Festlegungen bzw. Entwicklungen; im umfassendsten Konzept des International Network on Innovative Apprenticeship (INAP; http://www.inap.uni-bremen.de/) werden auch Innovationsprozesse und die Abstimmung mit dem Hochschulwesen einbezogen.

In den bisherigen Studien über die Übertragbarkeit der Lehrlingsausbildung haben sich ziemlich entmutigende Ergebnisse gezeigt, die mit der Komplexität dieser Ausbildungsinstitution, insbesondere der Verschränkung zwischen dem Bildungswesen und der Arbeitswelt bzw. den Arbeitsbeziehungen erklärt werden können (vgl. die Beiträge in Maurer \& Gonon 2014). Eine Konsequenz daraus besteht darin, dass sich neben dem klassischen systemischen Modell ein neuer Weg in der Definition der Lehrlingsausbildung entwickelt hat, der sich auf die Seite der Ausbildung konzentriert (während die Seite der Beschäftigung ausgeklammert oder nicht erwähnt wird) und entsprechend der These der Anpassung im empfangenden System - einer modularen Konzeption nahetritt, die im Transfer entsprechend der Einschätzung der Situation eine Auswahl von Komponenten vorschlägt. In den EUDokumenten findet man hier die beiden unterschiedlichen Ansätze vor (European Commission, Directorate-General for Employment, Social Affairs and Inclusion 2013 nimmt hier beispielsweise eine holistische Position ein, während andere Dokumente eher eine sehr weite Definition anwenden, die die Lehrlingsausbildung mehr oder weniger mit traineeship oder Formen von work-based learning identifizieren). Österreich, die Schweiz und Deutschland erfüllen die systemischen Kriterien im holistischen Sinn, wenn auch tw. mit beträchtlichen Unterschieden, die im Folgenden illustriert werden. 
Tab. 1: Zusammenstellung der Merkmale der Lehrlingsausbildung

\begin{tabular}{|c|c|c|}
\hline INAP 2013* & Steedman $2012\left(\right.$ ILO) ${ }^{* *}$ & ILO, G20, OECD*** \\
\hline \multicolumn{3}{|l|}{ BERUF } \\
\hline Occupational field & $\begin{array}{l}\text { Formal assessment } \\
\text { Recognized certification }\end{array}$ & $\begin{array}{l}\text { Established standards for } \\
\text { recognized occupation }\end{array}$ \\
\hline \multicolumn{3}{|l|}{ Shaping one's work } \\
\hline \multirow[t]{2}{*}{ Work context constitutive } & Workplace based & Based in the work place \\
\hline & On-the-job training & Fundamental aim learning \\
\hline Core occupations & & a trade/acquiring a skill \\
\hline \multicolumn{3}{|l|}{ Sustainable occ.profiles } \\
\hline \multicolumn{3}{|l|}{ Open dynamic occ.profiles } \\
\hline \multicolumn{3}{|l|}{ Occupational identity } \\
\hline \multicolumn{3}{|l|}{ STRUKTUR } \\
\hline & & intended for young people \\
\hline Time scale & Fixed duration & long-term training \\
\hline \multicolumn{3}{|l|}{ Cont.prof.development } \\
\hline Coop.learning venues & Off-the-job training & Off-the-job educ. \& training \\
\hline \multicolumn{3}{|l|}{ Legal status apprentices } \\
\hline \multirow[t]{2}{*}{ Cost-benefit for company } & & High level instruction \\
\hline & & Adequate remuneration \\
\hline \multicolumn{3}{|l|}{ Vocational disciplines } \\
\hline Integration in H.E.structure & & \\
\hline
\end{tabular}

\section{GOVERNANCE}

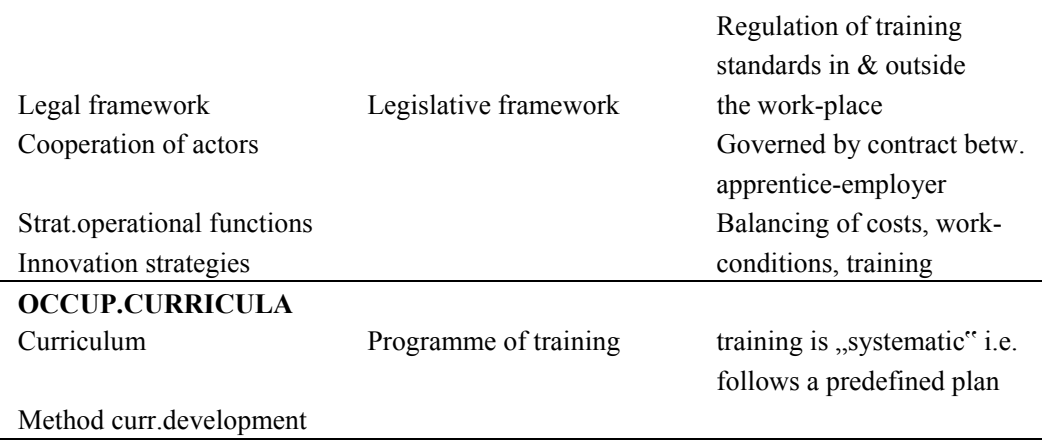

Quelle: eigene Zusammenstellung auf Basis von: *Memorandum, INAP-Commission, p.1-24 in Deitmer, Hauschildt, Rauner \& Zelloth 2013; ** Steedman 2012; ***ILO 1939-1962-2010 op.cit. Steedman 2012; G20 2012; OECD 2012 b. Sachlich ähnliche Merkmale wurden in den Zeilen der Tabelle jeweils parallel dargestellt. 


\subsection{Unterschiede in der Beteiligungsstruktur und der Einbindung der Lehrlingsausbildung in das Bildungswesen}

Insgesamt ist den drei betrachteten Ländern eine hohe Beteiligung an Berufsbildung gemeinsam. In einer Spannbreite der Beteiligung an Berufsbildung in den OECD-Ländern auf der Sekundarstufe zwischen 2\% und 74\% (OECD 43\%, EU 18\%) liegen Österreich mit 71\%, die Schweiz mit $65 \%$ und Deutschland mit 57\% teilweise im Spitzenfeld und jedenfalls deutlich über dem Durchschnitt (an 4., 7., 11. Stelle unter 36 Ländern). Österreich unterscheidet sich von den beiden anderen Ländern dadurch, dass die Berufsbildung besonders früh beginnt (lt. PISA befinden sich im Alter von 15 Jahren bereits $41 \%$ der Jugendlichen in der Berufsbildung, in der Schweiz nur 6\%, Deutschland keine Angabe).

Ein weiterer Unterschied wird zwischen den drei Ländern sichtbar, wenn man die Bildungsbeteiligung nach den beiden Altersgruppen der jungen Menschen (15-19 und 20-24 Jahre) differenziert und überdies auch die formal ausgeprägte Lehrlingsausbildung von der informalen Überschneidung zwischen Bildung, Beschäftigung und Arbeitslosigkeit unterscheidet. Diese Betrachtung aufgrund einer Tabelle in OECD Education at a Glance (vgl. OECD-EAG 2012) ${ }^{9}$ ergibt eine sehr aufschlussreiche Information über die vorhandenen Strukturierungen im internationalen Vergleich, die auch als Annäherung für Unterschiede der Tertiarisierung der Ausbildung interpretiert werden kann (siehe Tabelle 2).

Da normalerweise die Positionen Bildungsbeteiligung, Beschäftigung und Arbeitslosigkeit bzw. Nicht-Beschäftigung (out-of-labour-force) getrennt erfasst und dokumentiert werden (und sich teilweise auch institutionell ausschließen, wie formale Bildung und Arbeitslosigkeit), erscheinen sie auch als disjunkte Positionen. Die kombinierte Darstellung zeigt jedoch das hohe Maß an Überschneidung, vor allem zwischen Bildung und Beschäftigung, aber in manchen Ländern auch zwischen Bildung und Arbeitslosigkeit. Im OECDDurchschnitt macht die Kombination aus Bildung und Beschäftigung in beiden Altersgruppen mehr als 10\% aus, und die Kombination aus Bildung und Arbeitslosigkeit ergibt in der jüngeren Altersgruppe die Hälfte der gesamten Arbeitslosigkeit.

9 Indicator C5, Table C5.2a. Percentage 15-29 year-olds in education and not in education (2010). 
Tab. 2: Jugendliche in Bildung, Lehrlingsausbildung, und Kombinationen von Bildung mit Beschäftigung, Arbeitslosigkeit oder Nicht-Beschäftigung (in\%)

a.15-19-Jährige

\begin{tabular}{lllll}
\hline & Deutschland & Schweiz & Österreich & OECD (32) \\
\hline Lehrlingsausb. & 16,5 & 37,2 & 23,8 & 4,6 \\
Bi \& Besch. & 6,7 & 6,8 & 5,5 & 12,5 \\
Bi. \& ALos & 1,2 & 2,4 & 1,0 & 3,2 \\
Nicht Bi \& NB & 1,7 & 2,6 & 2,1 & 5,3 \\
Nicht Bi \& AL & 2,0 & 2,1 & 3,2 & 3,0 \\
\hline Bi insgesamt & 92,3 & 88,5 & 86,6 & 85,6 \\
RANG Bi insg. & 7. & 16. & 18. & $(19)$. \\
\hline
\end{tabular}

b.20-24-Jährige

\begin{tabular}{lllll}
\hline & Deutschland & Schweiz & Österreich & OECD (32) \\
\hline Lehrlingsausb. & 15,1 & 11,1 & 2,5 & 1,4 \\
Bi \& Besch. & 8,2 & 17,2 & 10,5 & 13,2 \\
Bi. \& ALos & 0,9 & 1,6 & 1,4 & 2,2 \\
Nicht Bi \& NB & 6,6 & 4,8 & 7,2 & 10,0 \\
Nicht Bi \& AL & 7,1 & 6,3 & 5,4 & 8,7 \\
\hline Bi insgesamt & 47,5 & 45,8 & 34,4 & 43,9 \\
RANG Bi insg. & 11. & 14. & 28. & $(16)$. \\
\hline
\end{tabular}

Quelle: eigene Abbildung und Berechnung aufgrund von OECD-EAG 2012; 1. Lehrlingsausb. (work-study programme); 2. Bi \& Besch.=in Bildung und Beschäftigung (education \& employment); 3. Bi. \& ALos=in Bildung und Arbeitslosigkeit (education \& unemployment); 4. Nicht Bi \& NB=nicht in Bildung und nicht in Beschäftigung (not in education \& not in the labour force); 5. Nicht Bi \& AL= nicht in Bildung und arbeitslos (not in education \& unemployed); 6. Bi insgesamt=in Bildung insgesamt incl. nur in Bildung ohne Überschneidung; 7. RANG Bi insg.=Rangplatz von in Bildung insgesamt unter 32 OECD-Ländern. Differenz auf 100\% sind verbleibende Kategorien (nur Beschäftigung, nur Arbeitslosigkeit, nur Nicht-Beschäftigung.

Hinsichtlich der gesamten Bildungsbeteiligung liegen Deutschland und die Schweiz über dem Durchschnitt, in Österreich ist diese jedoch niedriger, vor allem in der älteren Gruppe (nur Rang 28 unter 32 Ländern). Im Rangplatz der gesamten Bildungsbeteiligung steigt die Schweiz von der jüngeren zur älteren Gruppe um zwei Rangplätze (von 16. auf 14.), während Deutschland um 4 Plätze (von 7. auf 11.) und Österreich um 10 Plätze (von 18. auf 28.) zurückfallen. Vergleicht man die Lehrlingsausbildung (work-study programmes), so wird ein unterschiedliches Muster erkennbar, das auf die unterschiedliche Einbindung der Lehrlingsausbildung in die gesamten Bildungs- 
karrieren hinweist: In Deutschland sind je die Hälfte der Lehrlinge in der jüngeren bzw. älteren Altersgruppe, was den im Durchschnitt späteren Eintritt und die erweiterte Vorbildung in Form der Mittleren Reife oder des Abiturs spiegelt. In der Schweiz ist die Beteiligung in der jüngeren Altersgruppe deutlich erhöht, und es gibt auch einen nennenswerten Anteil an Lehrlingen von mehr als $10 \%$ in der älteren Altersgruppe. Die gesamte Bildungsbeteiligung in der älteren Gruppe wird v.a. durch die Lehre erhöht (zieht man diesen Anteil ab, so liegt diese in allen drei Ländern leicht über 30\%). Diese Strukturen können als Tendenz zur Tertiarisierung der Lehre interpretiert werden, während sich die Lehrlingsausbildung in Österreich fast vollständig auf die jüngere Altersgruppe konzentriert; darin drückt sich aus, dass die Tertiarisierung der Berufsbildung über die berufsbildenden Vollzeitschulen vonstattengeht. Die Lehre hat weitgehend das traditionelle Profil erhalten, das unmittelbar an die Pflichtschulbildung anschließt und auch oft mit geringeren Schulerfolgen verbunden ist.

Wie zu erwarten ist, liegt die informelle Kombination aus Bildung und Beschäftigung in der jüngeren Altersgruppe in den drei Vergleichsländern unter dem Durchschnitt (in der Größenordnung von über 5\%), in der Schweiz kommen die höheren beruflichen Ausbildungen in einer erhöhten kombinierten Beteiligung der älteren Gruppe zum Ausdruck.

Die Kombinationen von Arbeitslosigkeit bzw. Nicht-Beschäftigung liegen in den Vergleichsländern insgesamt deutlich unter dem OECD-Schnitt, in der jüngeren Gruppe deutlicher (um die Hälfte) als in der Älteren (bei zwei Drittel); in der Schweiz besteht ein Kontrast zwischen den Jüngeren mit erhöhten und den Älteren mit niedrigeren Werten, wobei v.a. die Kombination von Bildung und Arbeitslosigkeit erhöht ist, in Österreich liegt in der jüngeren Gruppe der Arbeitslosenanteil ohne Kombination sogar leicht über dem OECD-Schnitt.

Der Vergleich von Bildung und Arbeitslosigkeit mit dem EU-Schnitt unter Einbeziehung der 25-29-Jährigen zeigt zunächst eine deutlich erhöhte Bildungsbeteiligung unter den jungen Erwachsenen, ansonsten liegen die drei Länder im Schnitt (nur die 19-24-Jährigen in Österreich liegen darunter). Die Jugendlichen weder in Beschäftigung noch in Bildung liegen mit Ausnahme der jungen Erwachsenen in Deutschland mehr oder weniger deutlich unter dem EU-Schnitt, wobei die drei Länder ein unterschiedliches Profil zeigen: In Deutschland steigt dieser Anteil von einem sehr niedrigen Niveau in der jüngsten Gruppe stufenweise zum Schnitt an, in der Schweiz ist der Anteil in der jüngsten Gruppe etwas erhöht, in Österreich unter den jungen Erwachsenen - in zwei der drei Länder wird das ausgeprägt niedrige Niveau unter den 
Jugendlichen also nicht bis in das junge Erwachsenenalter aufrechterhalten (siehe Tabelle 3).

Tab. 3: Jugendliche und junge Erwachsene in Bildung, Arbeitslosigkeit und NichtBeschäftigung im Vergleich zu EU-21 (Index EU=100)

\begin{tabular}{llll}
\hline & Deutschland & Schweiz & Österreich \\
\hline BILDUNG & & & \\
15-19-J. & 103 & 99 & 97 \\
20-24-J. & 102 & 98 & 74 \\
25-29-J. & 119 & 112 & 114 \\
AL+NEET & & & \\
15-19-J. & 56 & 78 & 66 \\
20-24-J. & 75 & 65 & 65 \\
25-29-J. & 95 & 65 & 75 \\
\hline
\end{tabular}

Quelle: eigene Abbildung und Berechnung aufgrund von OECD-EAG 2012; BILDUNG=alle Kombinationen von Bildung; $A L+N E E T$ (neither in employment nor education/training) $=$ alle Kombinationen von Arbeitslosigkeit, Nicht-Beschäftigung und Nicht-Bildung.

\section{Arbeitsmarktpolitik und Arbeitsmarktentwicklung}

Hier geht es um Wirkfaktoren außerhalb der Lehrlingsausbildung. Dabei ist v.a. die wirtschaftliche Lage und Entwicklung zu beobachten, wo seit langem grundsätzlich zwei gegenläufige Tendenzen beobachtet werden: Einerseits ist der Jugendarbeitsmarkt ein Teil der Gesamtentwicklung und eine günstige wirtschaftliche Entwicklung beeinflusst auch den Jugendarbeitsmarkt positiv. Andererseits ist der Jugendarbeitsmarkt gleichzeitig sehr reagibel auf die wirtschaftlichen Schwankungen, und hier dreht sich die enge Verbindung der Lehrlingsausbildung zur employment relation, die grundsätzlich bessere Übergänge ermöglicht, ins Negative, indem der wirtschaftliche Abschwung auch direkt auf eine Verringerung der Lehrplätze wirkt. Eine wichtige Nebendingung in den letzten Jahrzehnten war auch die verbreitete demografische Konstellation, die mit dem Babyboom der 1960er Jahre gerade in den wirtschaftlich schwierigen Zeiten der frühen und mittleren 1980er Jahre einen relativen demografischen Überhang an Jugendlichen produzierte - dies hat sich inzwischen grundlegend gewandelt, indem diese starken Jahrgänge nun aus dem Erwerbsleben austreten was eher eine Übernachfrage nach jungen Menschen bewirkt.

In diesem Abschnitt werden zwei Phänomene betrachtet, erstens wird für eine ansatzweise Kontrolle der gesamten wirtschaftlichen Entwicklung ein 
Zeitreihen-Vergleich der Jugendarbeitslosigkeit und der Gesamtarbeitslosigkeit zum EU-Schnitt angestellt, und zweitens wird - ausgehend von ihrem starken Einsatz für Jugendliche in Österreich - die Rolle der Arbeitsmarktpolitik für Jugendliche in den drei Ländern verglichen.

Eine grundlegende Erwartung für die Systeme der Lehrlingsausbildung besteht darin, dass sie die Jugendarbeitslosigkeit reduzieren würden, die niedrigere Relation zwischen Jugendarbeitslosigkeit und Gesamtarbeitslosigkeit in Deutschland gegenüber allen G20-Ländern ist das wesentliche stilisierte Faktum, auf dem die Renaissance der Exportidee des deutschen Dualen Systems aufsetzt. Die zwei Vergleiche dieser Relation zeigen jedoch, dass Deutschland hier eine spezielle Stellung einnimmt (Abb.1). Die Jugendarbeitslosigkeit ist unter den drei Vergleichsländern nur in Deutschland relativ reduziert, in Österreich und der Schweiz liegt die direkte Relation der beiden Indikatoren nahe beim EU-Niveau (siehe Abbildung 1a), was sich auch indirekt darin ausdrückt, dass der Index von Jugendarbeitslosigkeit und Gesamtarbeitslosigkeit etwa in gleichem Maße vom jeweiligen EU-Niveau abweicht (siehe Abbildung 1b). Deutschland nimmt eine Sonderstellung ein, indem die Gesamtarbeitslosigkeit 2001-08 über dem EU-Niveau lag und auch die Jugendarbeitslosigkeit in den 2000ern ein deutlich höheres Niveau als in den beiden Vergleichsländern hatte, das jedoch 2005-11 eindrucksvoll reduziert wurde. In dieser Betrachtung wären im Wesentlichen die gleichen Ursachen für beide Formen der Arbeitslosigkeit verantwortlich, und es gibt keinen Grund, die Lehrlingsausbildung für das zwar absolut niedrigere, aber im Vergleich ähnliche Niveau der Jugendarbeitslosigkeit verantwortlich zu machen. 
Abb. 1: Relationen von Jugendarbeitslosigkeit (15-24-J.) und Gesamtarbeitslosigkeit (15-64-J.)

a. Index für Deutschland, Schweiz, Österreich: Gesamtarbeitslosigkeit=1,00

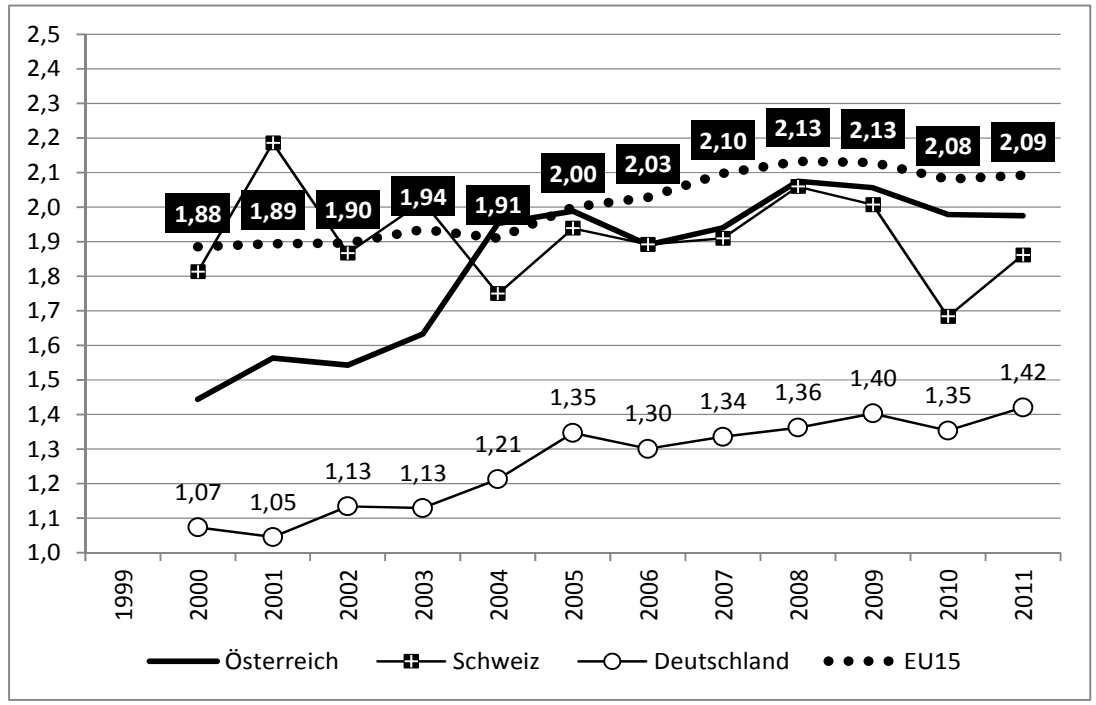

b. Index für Deutschland, Schweiz, Österreich im Vergleich zu EU: EU15=1,00

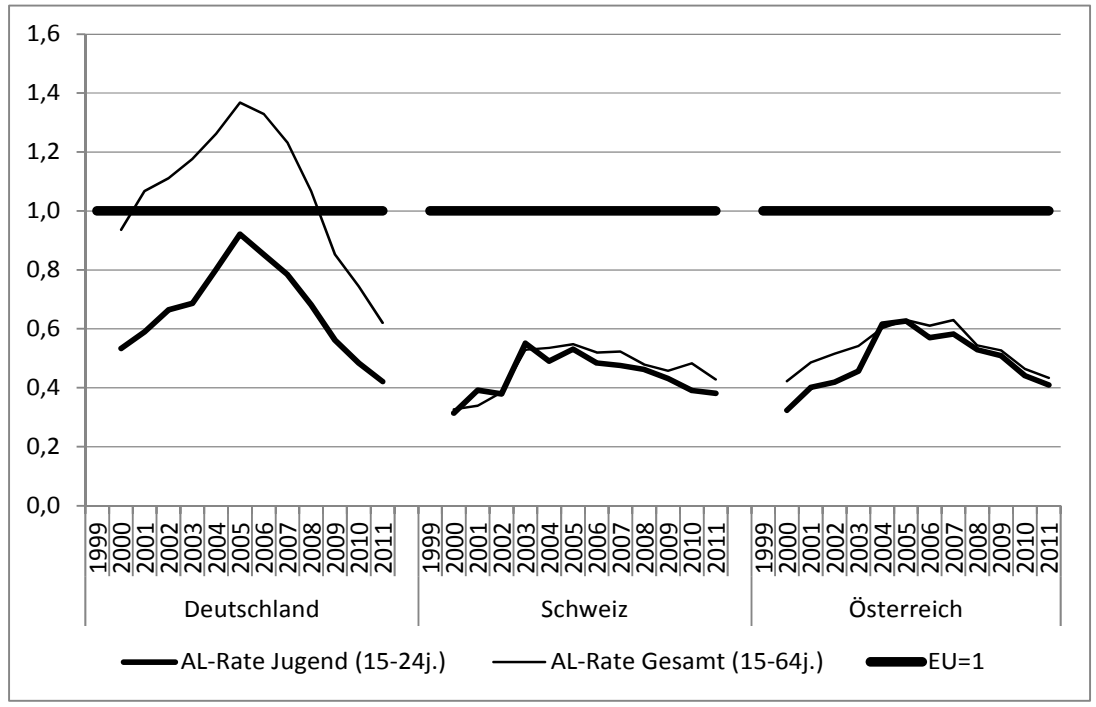

Quelle: eigene Abbildung und Berechnung aufgrund EUROSTAT. 
Der Einsatz der Arbeitsmarktpolitik für den Jugendarbeitsmarkt kann im Prinzip auf die employment relation zurückgeführt werden, die im Falle einer doppelten Konstruktion des Lehrverhältnisses (auch) als Arbeits- oder Beschäftigungsverhältnis einen Lehrlingsarbeitsmarkt konstituiert. Damit kann bei einem fehlenden Lehrplatz oder im Falle von Misserfolg bei der Lehrstellensuche auch der Status der Arbeitslosigkeit mit dem Recht auf Lohnersatzleistungen und arbeitsmarktpolitische Unterstützung begründet werden. Dies ist in Österreich sehr ausgeprägt institutionalisiert. Bereits mit den ersten wirtschaftlichen Krisenerscheinungen in den 1980ern wurden massive arbeitsmarktpolitische Jugendprogramme entwickelt und umgesetzt, die auch den Einsatz hochrangiger politischer Persönlichkeiten mobilisierte (vgl. Kreisky \& Svihalek 1989; Lassnigg 2016).

In Österreich kann dieser massive Einsatz der Arbeitsmarktpolitik in mehrfacher Hinsicht gezeigt werden: erstens werden betrieblich Lehrplätze in verschiedenen Formen gefördert, zweitens wurde die Lehrlingsausbildung aus Qualitätsgründen umstritten - in regulatorischer Hinsicht vielfach erleichtert, drittens wurde ein Programm der institutionellen Lehrlingsausbildung vor allem zur Förderung des Überganges kreiert (sog. Überbetriebliche Ausbildung, wo der Lehrlingsstatus vorübergehend oder manchmal auch bis zum Abschluss über eine Bildungsinstitution in Kombination mit betrieblichen Praktika oder manchmal auch eigenständigen Lehrwerkstätten konstituiert wird, viertens werden die verbleibenden arbeitslosen Jugendlichen in zusätzlichen arbeitsmarktpolitischen Programmen und Kursen vielfältig gefördert. Es werden 30-40\% der Ausgaben für aktive Arbeitsmarktpolitik für Jugendliche ausgegeben, die 15\% des Bestandes und 20\% des Zuganges in Arbeitslosigkeit ausmachen, obwohl Österreich über eines der umfangreichsten Berufsbildungssysteme in der OECD verfügt. Eine regionale Evaluierung der Jugendarbeitsmarktpolitik im wirtschaftlich dynamischsten Bundesland Oberösterreich hat ergeben, dass in einem gesamten Schul-Jahrgang bis zum Alter von 20 Jahren mehr als die Hälfte (mindestens) eine Vormerkung beim Arbeitsmarktservice hatten, und ein Viertel (26\%) bereits eine Förderung in Anspruch genommen hatten, darunter die Hälfte mehrfach mit bis zu 5 Teilnahmen. Wenn auch die nachhaltige Wirksamkeit dieser Programme teilweise begrenzt ist, so reduzieren sie jedenfalls deutlich die statistische Jugendarbeitslosigkeit, so dass die Jugendarbeitslosigkeit in Österreich - neben der vergleichsweise günstigen wirtschaftlichen Lage - insgesamt durch Interventionen in drei Säulen massiv bekämpft wird: Förderung der Lehrlingsausbildung, Förderung von Beteiligung im Schulwesen, Förderung durch arbeitsmarktpolitische Programme. Dies kann auch durch die Analyse der Zugänge in diese verschiedenen Bereiche im Zeitraum 2004-12, in dem der Einbruch stattgefunden hat, illustriert werden. Die stärksten Zugänge fanden in ar- 
beitsmarktpolitische Maßnahmen und in tertiäre und postsekundäre Ausbildungen statt (vgl. Lassnigg 2013). Der grundständige Beitrag der Lehrlingsausbildung als solcher ist eher indirekt, als Auslöser für die verschiedenen anderen Maßnahmen, zu sehen.

Die Frage, ob dieser Mechanismus auch in den beiden anderen Ländern gefunden werden kann wurde durch die Recherche von vergleichenden Daten der Arbeitsmarktpolitik in einem ersten Schritt geprüft. Dabei hat sich insgesamt herausgestellt, dass der Einsatz von arbeitsmarktpolitischen Maßnahmen für Jugendliche im Zeitverlauf nur sehr spärlich beobachtet wird (in EUROSTAT werden Jugendliche nicht gesondert berichtet). In der OECD Datenbasis über Arbeitsmarktpolitik sind Informationen über die Förderung der Lehrlingsausbildung in Form von Ausgaben/BIP (Schweiz nur bis 2007) und Personen/Arbeitskräftepotential verfügbar. Insgesamt liegen die Ausgaben der drei Länder für Arbeitsmarktpolitik über dem OECD-Durchschnitt, die Beteiligung beim Durchschnitt bzw. für die Schweiz darunter. Die Angaben für die Lehrlingsausbildung ergeben hier für Österreich einen starken Anstieg in den beiden Krisenjahren 2007-08 (14\% aller Ausgaben für aktive Arbeitsmarktpolitik und $20 \%$ der geförderten Personen), dies gilt jedoch nicht für die beiden anderen Länder: in der Schweiz werden keine Ausgaben für Lehrlinge berichtet, in Deutschland liegt das Niveau der Förderung ähnlich wie in Österreich vor der Krise 2004-05, mit einer leichten Tendenz zur Steigerung, zeigt jedoch nicht diesen sprunghaften Anstieg wie in Österreich (siehe Abbildung 2). Die These, dass die Lehrlingsausbildung über die employment relation in die Arbeitsmarktpolitik eingebunden ist, kann also nicht von Österreich auf die anderen Länder generalisiert werden.

\section{Schlüsse, Fragen}

Im Rahmen der komplexen Gestaltungsformen der Lehrlingsausbildung zeigen (wie bereits in Busemeyer \& Trampusch 2011 herausgearbeitet wurde) auch die klassischen Systeme im deutschsprachigen Raum wesentliche Unterschiede. Dies wird mit quantitativen Indikatoren untermauert, indem die unterschiedliche Beziehung der Berufsbildung zur Tertiarisierung gezeigt wird, mit dem deutschen dualen System auf dem einen Pol, und dem österreichischen dualistischen System der Parallelität von (höheren) berufsbildenden Schulen und Lehrlingsausbildung auf dem anderen Pol. Weitere Unterschiede betreffen unterschiedliche Aufwands-Ertrags-Relationen, die für Schweiz und Deutschland viel besser untersucht sind. In Österreich ist bisher 
Abb. 2: Ausgaben und Personen in der Arbeitsmarktpolitik: Deutschland, Schweiz, Österreich im Vergleich zum OECD-Durchschnitt

a. Aktive Arbeitsmarktpolitik (AMP) insgesamt (Index: $O E C D=1,00)$

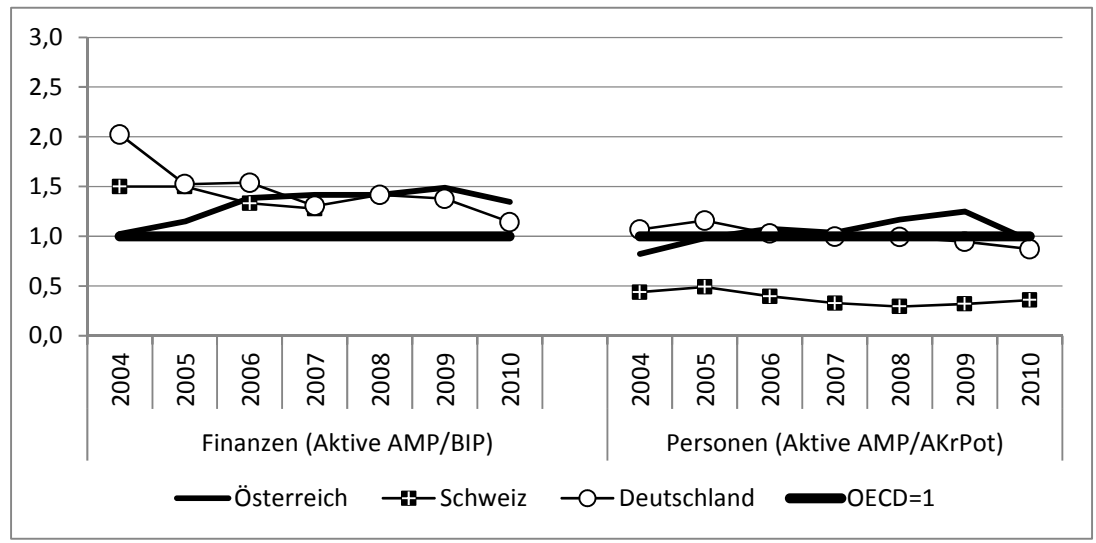

b. Förderung von Lehrlingen als Anteil aktiver AMP (Index: $O E C D=1,00$

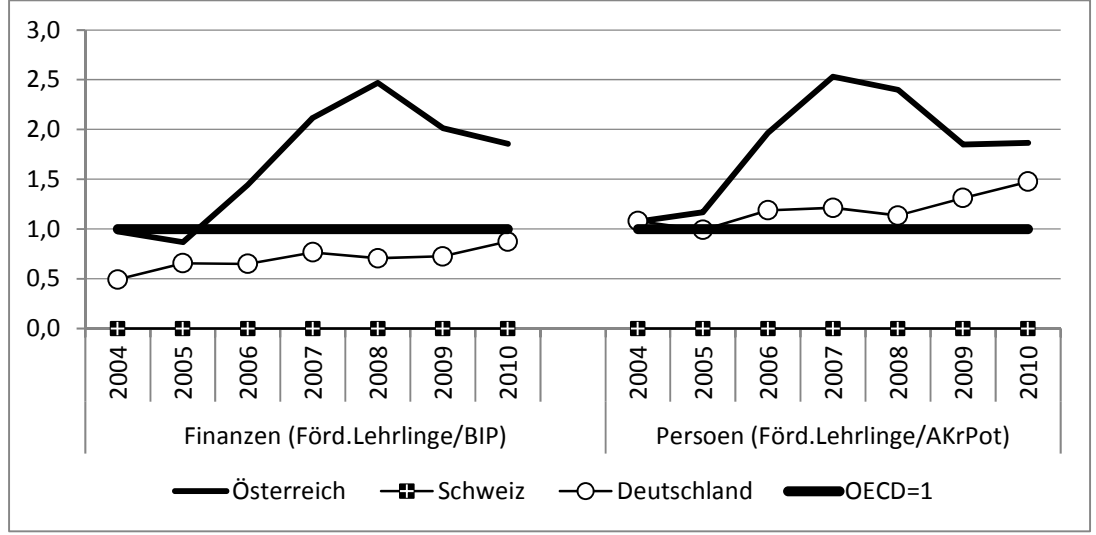

Quelle: eigene Abbildung und Berechnung aufgrund OECD; die Ausgaben sind auf das jeweilige BIP, und die Personen auf das jeweilige Arbeitskräftepotential (AKrPot) standardisiert

nur eine diesbezügliche Untersuchung aus den 1990er Jahren verfügbar mit im Durchschnitt der Betriebe geringen Nettokosten der Ausbildung, während in der Schweiz im Durchschnitt Nettoerträge lukriert werden und in Deutschland vor allem in den ersten Untersuchungen die Betriebe hohe Nettokosten zu tragen hatten (vgl. Lassnigg \& Steiner 1997; Lassnigg 2008). Diesen Unterschieden entspricht in Österreich ein hohes Ausmaß an work-based learn- 
ing das am unteren Ende des Qualifikationsspektrums angesiedelt ist, während in Deutschland der Anteil an höherwertigen Ausbildungen (Abitur als Zugang) größer ist und in der Schweiz die überbetriebliche-institutionelle Komponente und die Verbindung mit der tertiären Ebene viel stärker ausgeprägt ist.

Dem wesentlichen Argument aus der globalen politischen Debatte zum Export der Lehrlingsausbildung, dass durch dieses Arrangement die Jugendarbeitslosigkeit verringert würde, wird am Beispiel Österreichs und der beiden Vergleichsländer näher nachgegangen. Es ergeben sich einige stark relativierende Befunde:

- Die Jugendarbeitslosigkeit ist in relativen Begriffen nur in Deutschland systematisch niedriger als die Erwachsenenarbeitslosigkeit, in der Schweiz und in Österreich gibt es diesen Unterschied nicht und die niedrige Jugendarbeitslosigkeit scheint vor allem durch die günstige Wirtschaftslage beeinflusst zu sein.

- In allen drei Ländern gibt es v.a. seit den 2000er Jahren Probleme mit einer ausreichenden Anzahl an Lehrstellen und an Arbeitsplätzen für junge Menschen, die unterschiedliche Konfigurationen annehmen: In der Schweiz liegen die Probleme eher bei der jüngeren Gruppe der 15-19Jährigen, während sich in Österreich und Deutschland eher Probleme im Anschluss an die Jugendphase bei den über-25-Jährigen zeigen.

- In Österreich haben neben der größeren Rolle der schulischen Berufsbildung im „dualistischen System“ (insbesondere in der wirtschaftlichen Krise seit 2008) die intensiven Interventionen der Arbeitsmarktpolitik als wesentlicher Faktor zur (kurzfristigen) Verringerung der Jugendarbeitslosigkeit gewirkt, in den beiden anderen Ländern jedoch nicht. Die Hypothese der Wirkungen der employment relation auf die stärkere Einbindung der Lehrlingsausbildung in die Arbeitsmarktpolitik muss daher zurückgewiesen werden.

Abschließend können einige Botschaften und Fragen aus diesen Analysen zusammengefasst werden. Erstens ist im Hinblick auf die Wirkungen dieser Ausbildungsform die Frage festzuhalten, ob es sich um ein holistisches System oder ein loses Konglomerat von Merkmalen handelt. Vieles spricht dafür, dass die Wirksamkeit sich aus dem komplexen systemischen Zusammenhang der vielfältigen Merkmale ergibt, während sich die aktuelle Export- und Transfer-Politik eher an der Vorstellung eines modularen Konglomerats von Merkmalen orientiert - dieser Zugang wäre eher als unwirksame symbolische Politik zu interpretieren, die komplexe systemische Interpretation erschwert jedoch aufgrund der hohen Anforderungen den Export bzw. Transfer. Zweitens erscheint eine balancierte Sicht der wirtschaftlichen (Lohn, employment 
relation) und der pädagogischen (Lernorganisation) Elemente in der Modellierung wichtig, um die Wirkungen dieser Ausbildungsform zu verstehen auch im wissenschaftlichen Diskurs werden diese jedoch u.a. aufgrund unterschiedlicher disziplinärer Zugänge etwa zwischen Ökonomie und Berufspädagogik tendenziell voneinander isoliert, was das Verständnis erschwert.

In methodischer und forschungsstrategischer Hinsicht erscheint die OECD-Auswertung und Darstellung der Überschneidungen zwischen Bildung, Arbeitslosigkeit und Beschäftigung, sowie die Altersdifferenzierung der Jugendlichen und jungen Erwachsenen nach den verschiedenen Karrierephasen eine wichtige Betrachtungsperspektive zu geben, die weitergeführt und vertieft werden sollte. Die Dichotomien zwischen sekundärer und tertiärer Ausbildung, wie auch zwischen (nur) Bildung und (nur) Beschäftigung, die die Diskurse teilweise blockieren, können damit in eher graduelle Phänomene aufgelöst werden. Die einfache Betrachtung ergibt bereits ein gutes Vergleichsbild der drei Länder-Strukturen und fördert eine Prozessbetrachtung. Der (relative) Vergleich von Erwachsenen- und Jugendarbeitsmärkten anstelle der verbreiteten isolierten Betrachtung erscheint instruktiv und stellt auf sehr einfache Weise die direkten Wirkungen der Lehrlingsausbildung auf die Jugendarbeitslosigkeit in Frage, in der gegenwärtigen Betrachtung zumindest für die Schweiz und Österreich, aber in letzten Jahren haben sich die Indikatoren auch in Deutschland angenähert; damit bricht das wichtigste Export/Transfer-Argument zusammen. Die Unterscheidung der Wirkungen, die sich aus der employment relation ergeben, im Vergleich mit den Wirkungskomponenten aufgrund der Gelegenheit zum work-based learning und aufgrund der Berufs-Zertifizierung ist als eine wichtige offene Forschungsfrage im Vergleich mit loseren informellen Formen der Verbindung von Bildung und Beschäftigung hervorzuheben.

\section{Literatur}

Busemeyer, M.R. \& Trampusch, C. (Eds.). (2011). The Comparative Political Economy of Collective Skill Systems. Oxford: Oxford University Press.

Deitmer, L., Hauschildt, U., Rauner, F. \& Zelloth, H. (Eds). (2013). The architecture of innovative apprenticeship. Dordrecht: Springer.

EC-European Commission, Directorate-General for Employment, Social Affairs and Inclusion (2012). Apprenticeship supply in the Member States of the European Union. Final report. Luxembourg: Publications Office of the European Union. 
European Commission, Directorate-General for Employment, Social Affairs and Inclusion. (Eds.). (2013). Apprenticeship and traineeship schemes in EU27: Key Success Factors: A Guidebook for Policy Planners and Practitioners. Online: http://ec.europa.eu/social/BlobServlet?docId=11348\&langId=en (16.02.2016).

Fuller, A. \& Unwin, L. (Hrsg.). (2013). Contemporary Apprenticeship. International Perspectives on an Evolving Model of Learning. Abingdon: Routledge.

G20 (2012). G20 Labour and Employment Ministers' Conclusions 17-18 May 2012, Guadalajara, Mexico. Online: http://www.g20.org/English/Documents/Past Presidency/01512/P020151225641497684815.pdf (16.02.2016).

Greinert, W.-D. (2005). Mass vocational education and training in Europe. Classical models of the 19th century and training in England, France and Germany during the first half of the 20th. Cedefop Panorama series 118. Luxembourg: Office for Official Publications of the European Communities. Online: https://cumulus.cedefop.europa.eu/files/vetelib/eu/pub/cedefop/pan/2005_5157_en.pdf $\quad(16.02 .20$ 16).

Kreisky, B. \& Svihalek, F. (Hrsg.). (1989). Achtung - Fertig-Arbeitslos. Zur Situation jugendlicher Arbeitsloser. Wien: Österr. Staatsdruckerei.

Lassnigg, L \& Steiner, P.M. (1997). Die betrieblichen Kosten der Lehrlingsausbildung. Materialien zu Wirtschaft und Gesellschaft Nr. 67. Wien: Kammer für Arbeiter und Angestellte. Online: http://www.equi.at/dateien/lehrl_betrkozusfass.pdf (16.02.2016).

Lassnigg, L. (2008). Costs - benefits - quality: the specific profile of Austrian apprenticeship and its future potentials and drawbacks. In P. Schlögl, F. Rauner, P. Grollmann \& E. Smith (Eds.), Situated competence development through innovative apprenticeships. The role of different stakeholders. INAP Conference Proceedings 01-02 Feb. 2008, Vienna, (S.163-169). Bremen: ITB. Online: http://www.equi.at/dateien/inap-apprenticeship-pdf.pdf (17.02.2016).

Lassnigg, L. (2011). The 'duality' of VET in Austria: institutional competition between school and apprenticeship. Journal of Vocational Education and Training, 63(3), 417-438.

Lassnigg, L. (2013). Austria's success on the youth labour market - not systemic but voluntaristic. European Lifelong Learning Magazine (Elm), (1). Online: http:// www.elmmagazine.eu/articles/what-are-they-doing-right-3-cases (17.02.2016).

Lassnigg, L. (2015). The political branding of apprenticeship into the 'Dual System': Reflections about exporting the myth of employment transition. In A. Heikkinen \& L. Lassnigg (Eds.), Myths and Brands in Vocational Education, (S. 78-98). Newcastle upon Tyne: Cambridge Scholars Publishing.

Lassnigg, L. (2016) Apprenticeship policies in comparative perspective. ETstructures, employment relationship, export. IHS Reihe Soziologie 114. Wien: Institut für Höhere Studien. Online: http://www.ihs.ac.at/fileadmin/ public/soziologie/pdf/rs 114.pdf (17.02.2016).

Maurer, M. \& Gonon, P. (Eds.). (2014). The challenges of policy transfer in vocational skills development. Bern: Peter Lang.

Mazenod, A. (2015). The myth of ,apprenticeship“: comparing Finland, France and England. In A. Heikkinen \& L. Lassnigg (Eds.), Myths and Brands in Vocational Education, (S.190-204). Newcastle upon Tyne: Cambridge Scholars Publishing.

Mazenod, A. (2016). Education or training? A comparative perspective on apprenticeships in England. Journal of Vocational Education \& Training, 68(1), 102-117. 
OECD (2012a). The challenge of promoting youth employment in the G20 countries (May). Online: http://www.oecd.org/employment/emp/50304960.pdf (14.02. 2016)

OECD (2012b). OECD Note on 'quality apprenticeships' for the G20 task force on employment (September). Paris: OECD. Online: http://www.oecd.org /els/emp/ OECD\%20Apprenticeship\%20Note\%2026\%20Sept.pdf (16.02.2016)

OECD-EAG (2012). Education at a Glance. Paris: OECD.

Steedman, H. (2012). Overview of apprenticeship systems and issues. ILO contribution to the G20 Task Force on Employment (November). International Labour Office, Skills and Employability Department - Geneva: ILO. Online: http:// www. ilo.org/wcmsp5/groups/public/@ed_emp/@ifp_skills/documents/genericdocume nt/wcms_190188.pdf(16.02.2016). 


\section{Die Berufsbildung der Schweiz als permanenter Kompromissbildungsprozess}

\section{Philipp Gonon, Lea Zehnder}

Berufsbildung ist aus dem Blickwinkel einer Steuerungsperspektive in der Regel ein ausgehandelter Kompromiss unterschiedlicher Anliegen. ${ }^{1}$ Staatsbürgerliche, berufsfachliche, ökonomische und soziale Interessen finden in der Organisation und Durchführung der beruflichen Bildung ihren Niederschlag. Verschiede Akteure bzw. Interessenvertreterinnen und Interessenvertreter sind angehalten, eine Lösung, die mit Abstrichen für alle akzeptabel ist, zu finden. Dieser Ansatz wird im Folgenden anhand eines konventionstheoretischen bzw. rechtfertigungssoziologischen Approachs im Zusammenhang mit der Entstehung eines nationalen Berufsbildungsgesetzes und der Einführung einer Berufsmaturität in der Schweiz durchgespielt. Das Ergebnis solcher Aushandlungen ist eine Hybridisierung des regulativen Arrangements. Für eine solche Darstellung eignen sich die Debatten im Zusammenhang mit den Rechtssetzungsprozessen rund um die Berufsbildung, die in der Schweiz relativ früh zu verorten sind. Im ersten Teil des Beitrags wird der Verlauf der Gesetzgebung rekonstruiert und im zweiten Teil wird dann diese Perspektive der Konfliktlösung und Kompromissbildung auf eine jüngere Reform, nämlich die Berufsmaturität in der Schweiz angewendet.

\section{Konventionstheoretische Rahmung der Berufsbildung}

In den letzten Jahren haben pfadabhängige Darstellungen, häufig entstanden aus dem von den Poliltikwissenschaften inspirierten historischen Institutionalismus, die Debatte um Berufsbildungsentwicklung und Berufsbildungsreformen geprägt. Regulierungen und Gesetzgebungen für die berufliche Bildung sind Ausgangspunkte für weiterführende Entwicklungen, die dem Berufsbildungssystem beachtliche Stabilität und dennoch auch Wandlungsfähigkeit zusprechen.

Für die Bundesrepublik Deutschland hat beispielweise Busemeyer den „Wandel trotz Reformstau“ beschrieben, der diese Entwicklung seit dem ersten Berufsbildungsgesetz 1969 darstellt (vgl. Busemeyer 2009). Näher am Reformprozess bzw. an den Debatten rund um die Entstehung der Berufsbil-

1 Grundlage dieses Beitrages ist ein vom Schweizerischen Nationalfonds gefördertes Projekt „Von der Meisterlehre zur dualen Berufsbildung“, das am Lehrstuhl für Berufsbildung, Universität Zürich, unter Beteiligung der Autoren durchgeführt wird. 
dungsgesetzgebung war bereits Claus Offes „Berufsbildungsreform - Eine Fallstudie über Reformpolitik“. Er analysierte als Zielsetzung der politischen Steuerung die „Integration“, die zu einem Ausbau des Variationsbereiches möglicher Resultate führen sollte, das heißt eine staatlich initiierte Institutionalisierung, die auf eine „Erweiterung von Optionen durch Beseitigung von Partikularismen“" setzte (vgl. Offe 1975, 33 \& 43).

\subsection{These und Perspektive}

Im Folgenden versuchen wir diese These der Integration verschiedener Anliegen durch die Kompromissfindung in Form einer Hybridisierung zu plausibilisieren. Dieser Prozess erfolgte bereits im Rahmen der Institutionalisierung von Berufsbildung in der Schweiz in den 1930er Jahren. In einem zweiten Schritt soll diese These weiter anhand der Begründung der Berufsmaturität in den 1990er Jahren wieder aufgegriffen werden. Hierbei werden Akteure und ihre Interessen in den Fokus gerückt. Diese vertreten Werte, die sie im Rahmen eines Gesetzgebungsprozesses artikulieren und gegenüber Dritten rechtfertigen. Dabei wollen wir uns an der Konventionentheorie der pragmatischen Soziologie orientieren. Diese sieht Konventionen und die damit zusammenhängenden Qualitätsansprüche als ein situatives Koordinationsdispositiv (vgl. Neu 2015). Gemäß der Soziologie der Konventionen sind Akteure, die in Wirtschaft und Gesellschaft Entscheidungen treffen und durchsetzten wollen, einem Rechtfertigungsimperativ unterworfen (vgl. Boltanski \& Thévenot 2007). Ihre Interessen müssen gegenüber Dritten gerechtfertigt werden, auf möglich oder tatsächlich erfolgte Kritik muss reagiert werden. Hierbei bestehen gleichzeitig verschiedene Ansprüche, die unterschiedliche Werte repräsentieren (vgl. Diaz-Bone 2011).

Die Entwicklung der Berufsbildungsgesetzgebung in der Schweiz lässt sich anhand solcher Rechtfertigungen darlegen, die als Kompromisse handwerkliche, industrie- und marktbezogene, aber auch öffentliche (und weitere) Wertigkeiten hierarchisieren und als Konventionen bzw. Konventionskompromisse etablieren. Gemäß der Konventionssoziologie gäbe es noch weitere Ansprüche und Wertigkeiten, nämlich die Inspiration, das Projekt und die öffentliche Meinung, die aber im Folgenden nicht weiter berücksichtigt werden, da wir uns auf die in den Dokumenten und Stellungnahmen zur Berufsbildungsreform dominant bzw. am häufigsten genannten Konfliktlinien und Begründungsmuster konzentriert haben (siehe Tabelle 1). 
Tab. 1: Konventionsbasierte Rechtfertigungen \& akteursbezogene Ansprüche

\begin{tabular}{lcc}
\hline Konvention & Koordinationsprinzip & Qualitätsanspruch \\
\hline Markt & Preis, Tausch & Wettbewerbsfähigkeit \\
Industrie & Funktionalität & Effizienz \\
Handwerk & Vertrauen (Gemeinschaft) & $\begin{array}{c}\text { Reputation, Tüchtigkeit } \\
\text { Staatsbürgertum }\end{array}$ \\
& Rechtsgleichheit & $\begin{array}{c}\text { Kollektiver Anspruch, } \\
\text { rechtlicher Schutz }\end{array}$ \\
(Inspiration) & (Freie Zirkulation) & (Kreativität) \\
(Öffentliche Meinung) & (Mediale Vermittlung) & (Sichtbarkeit, Popularität) \\
(Projekt) & (Vernetzung) & (Flexibilität, Offenheit) \\
\hline
\end{tabular}

Quelle: eigene Darstellung in Anlehnung an Boltanski \& Thévenot 2007.

Es sind Qualitätsansprüche, die eine vormals angesehene Einrichtung handwerklich-kaufmännischer Ausbildung aus dem historischen Niedergang und Tief zu Beginn des 20. Jahrhunderts quasi herauszuholen trachteten. Mit Überlegungen, die den kollektiven Anspruch und den Schutz der Lehrlinge hervorhoben, wie auch mit effizienz- und wettbewerbsorientierten Forderungen sollte unter anderem eine gesamtschweizerische Regulierung und der Einbezug von Schule in die berufliche Ausbildung bewerkstelligt werden. Die Konventionen des Marktes, der Industrie, des Handwerks und des Staatsbürgertums sollten im Sinne eines Sowohl-Als-Auch in ein entsprechendes Berufsbildungsgesetz integriert werden.

\subsection{Historische Akteure der Berufsbildung}

Die treibenden Kräfte im Bezug auf die Entwicklung und den Aufbau der Berufsbildung in der Schweiz waren der Schweizerische Gewerbeverband und später auch die Schweizer Gewerkschaften. Die vom Bundeskomitee des Schweizerischen Gewerkschaftsbundes 1912 veröffentlichte „Eingabe betreffend ein Eidgenössisches Lehrlingsgesetz" weist bereits in ihrer Betitelung darauf hin, dass es zentral um den Status des Lehrlings geht, der unter veränderten industriell geprägten Verhältnissen gegenüber der un- und angelernten Arbeitskraft Anspruch auf eine qualitativ hochstehende Ausbildung, bzw. eine ,gute Berufslehre“ habe.

Im Unterschied zum Gewerbe, das im Interesse eines „leistungsfähigen Gewerbestandes“ in erster Linie die Berufstüchtigkeit anvisiere, ginge es 
„unter industriell dominierten und kapitalistischen Bedingungen“, so der Gewerkschaftsbund, auch um den ausreichenden Schutz für die Arbeitskraft des Lehrlings. Gegenüber vorgängigen Gesetzesentwürfen bestand der gewerkschaftliche Vorschlag darin, ein Mindestalter für den Beginn der Lehre festzuhalten und eine lohnbezogene und organisatorische Gleichartigkeit der beruflichen Ausbildung einzufordern. Darüber hinaus gelte es die Bundesaufsicht zu stärken, aber auch paritätische und regionale Aufsichtskommissionen für die Durchführung des Lehrlingsgesetzes zu installieren. Vor allem müsse vermieden werden, dass junge Leute wie Lehrlinge behandelt würden, die eigentlich keine seien, wie am Beispiel des Verkaufes erläutert wurde: In einigen Betrieben würden junge Frauen als „Lehrtöchter“ für ein geringes Entgelt eingestellt und bis zu zwei Jahren verpflichtet, ehe sie dann als ,angehende Verkäuferinnen“" weiterhin zu einem tieferen Lohn als in Ausbildung Befindliche arbeiteten, während in anderen Unternehmen die gleichen Dienste in vier bis sechs Wochen erlernt würden, um dann als ausgelernte Verkäuferinnen behandelt zu werden (vgl. SGB 1912, 5ff.). Es waren vor allem Vorbehalte bezüglich des Lernortes Betrieb, die bei Arbeitnehmervertretern eine gewisse Zurückhaltung und Skepsis aufkommen ließen, berufliche Bildung weitgehend unbeaufsichtigt den Unternehmern zu überlassen (vgl. Bernet 1923).

Auch die 1918 publizierten Vorarbeiten zu einer gesamtschweizerisch ausgerichteten gesetzlichen Regelung des Schweizerischen Gewerbeverbandes hielten für die berufliche Bildung einen Reform- und Förderungsbedarf fest. Der Entwurf eines entsprechenden „Bundesgesetzes betreffend Berufslehre und Berufsbildung" war ursprünglich als Bestandsteil einer Schweizerischen Gewerbegesetzgebung vorgesehen. Dabei spielte u.a. auch die starke Gewichtung des Handwerks eine Rolle. So wurde mit der 1905 erfolgten eidgenössischen Betriebszählung argumentiert, die darlege, dass die Großindustrie „deutlich weniger Lehrlinge“ halte als die Handwerke. Das Handwerk müsste allerdings nicht nur sich selbst, sondern auch für die Großindustrie tüchtige Arbeiter erziehen (vgl. SGV 1918). Das neu zu schaffende Berufsbildungsgesetz sei daher auf alle Berufe - mit Ausnahme der Landwirtschaft und der wissenschaftlichen Berufe - auszuweiten; so sollten namentlich auch die Industrie und die Fabrikbetriebe einbezogen werden, gerade auch um Willkür und „Unbilligkeiten“ zu vermeiden (vgl. ebd., 27). Die Lehrlingsausbildung beruhe anders als zu Zeiten der Zünfte nicht mehr auf einer gemeinsam zu tragenden Pflicht der Berufsgenossenschaft, sondern sei neuerdings lediglich ein persönlicher Arbeitsvertrag geworden (vgl. ebd., 13). Die mangelnde Qualität, bzw. die „Lücken und Mängel“ der beruflichen Bildung erforderten, dass Staat und Gesellschaft sich ernstlich verpflichten müssten 
im Interesse des Erhalts eines soliden Mittel- und Arbeiterstandes aber auch im Sinne des Nationalwohlstandes und der Konkurrenzfähigkeit für die berufliche Tüchtigkeit Sorge zu tragen. Neben den postulierten Maßnahmen sei freilich auch die Selbsthilfe bedeutsam (vgl. ebd., 27).

\section{Erste gesamtschweizerische Berufsbildungsgesetzgebung 1930 als pfadabhängige Setzung}

Mit den von Gewerkschaften und Gewerbe genannten auf Staat und Markt, Industrie und Handwerk ausgerichteten Qualitätsansprüchen - Aufsichts-, Schutz- und Erziehungsgedanke einerseits, handwerklich geprägte Berufstüchtigkeit andererseits - ist der argumentative Resonanzboden der später entwickelten Berufsbildungsgesetze benannt. Auch die Industrie- und exportorientierten Verbände, die später sich in diesen Diskurs einbrachten, bezogen sich auf diese im Vordergrund stehenden Konventionen, betonten aber aus ihrer Warte vor allem die funktionellen Erfordernisse, welche die Wirksamkeit der Gesetzgebung gewährleisten sollte (vgl. Berner, Gonon \& Ritter 2011; Imdorf, Berner \& Gonon 2015).

Die Akteure der ersten Stunde, vor allem der Gewerbeverband, wiesen in ihren Ausführungen öfters auf den früheren Stand der Ausbildung in vergangenen Zeiten hin, nämlich auf die berufliche Bildung in den Zünften. Die Botschaft des Bundesrates aus dem Jahre 1928 nahm diesen Faden wieder auf und stellte nach der Abschaffung der Zünfte in der Schweiz einen ,Tiefstand der beruflichen Ausbildung" fest, den es durch eine entsprechende Gesetzgebung zu beheben gelte (vgl. Schweizerischer Bundesrat 1928, 725). Entgegen der ursprünglichen Absicht rang sich der Gewerbeverband durch, die anderen Gewerbeförderungsanliegen hintanzustellen und sich zunächst auf die Berufsbildungsgesetzgebung, basierend auf dem Vorentwurf des von der Bundesverwaltung eingerichteten Arbeitsamtes, $\mathrm{zu}$ fokussieren. Die Notwendigkeit einer bundesgesetzlichen Regelung der beruflichen Bildung wurde darüber hinaus auch aufgrund eines festgestellten Facharbeitermangels begründet (vgl. ebd., 733).

Außerdem wurde dezidiert darauf hingewiesen, dass dieses Gesetz keinesfalls nur für Handwerksbetriebe gelten sollte, wie dies im ursprünglich deutsch verfassten Text anklinge; vielmehr sei es klar so zu handhaben wie es in der italienischen und französischen Version stehe, welche explizit unter dem Begriff „Industrie“ - diese einschließend - über das Gewerbliche hinausginge (vgl. ebd., 736). 
Auf die Kritik von Seiten des Schweizerischen Gewerkschaftsbundes, vom Christlich-Sozialen Arbeiterbund und vom Kaufmännischen Verband, dass der Lehrlingsschutz in der vorliegenden Gesetzgebung zu wenig präsent sei, ging der Bundesrat aber nicht ein (vgl. ebd., 729). Der Lehrlingsschutz sei weggelassen worden, da dieser in anderen Gesetzen, so in kantonalen Lehrlingsgesetzen, geregelt sei. Der Schwerpunkt des Gesetzes sei die umfassende Förderung aller Bereiche der Berufsbildung, inklusive die Anlernung, die Lehrabschlussprüfung, die Meisterprüfung und höhere Fachprüfung (vgl. ebd., 739).

In der Ausarbeitung des Berufsbildungsgesetzes ging es darüber hinaus darum, die Rolle des Bundes und der Kantone zu klären. Dem Bund wurde eine Oberaufsicht zugesprochen, im Zentrum standen aber die kantonalen Behörden, welche den Vollzug des Berufsbildungsgesetzes in der Hand haben sollten. Daneben seien es die Berufsverbände und die Handwerksvertreter, welche mit Billigung der Arbeitnehmenden, vorwiegend die betrieblichen Teil der Ausbildung regulieren, sowie Prüfungen durchführen sollten (vgl. Schweizerischer Bundesrat 1928, 742).

Als unhintergehbares Kernelement für die Anerkennung einer Ausbildung als Berufsbildung wurde außerdem der verpflichtende Tagesschulbesuch des beruflichen Unterrichts festgelegt, der in der Folge systematisch ausgebaut wurde (vgl. Schweizerischer Bundesrat 1962).

Mit dem ersten gesamtschweizerischen Berufsbildungsgesetz aus dem Jahre 1930, welches in erster Linie aber nicht ausschließlich von den gewerblichen Qualitätsansprüchen geprägt war, etablierten Wirtschaftsverbände und Sozialpartner sowie staatliche Akteure ein Regime, das Kompromisse und dennoch die Koexistenz unterschiedlicher Werte zuließ. Die nachfolgenden Gesetzesrevisionen 1963, 1978 und 2002 erweiterten und differenzierten in einem Öffnungs- und Schließungsprozess diese erste bundesstaatliche Institutionalisierung der Berufsbildung.

Das Berufsbildungsgesetz aus dem Jahre 1963 sprach nun von Berufsbildung, und nicht mehr von beruflicher Ausbildung, um damit festzuhalten, dass einerseits die „,charakterliche Erziehung des Lehrlings“ (vgl. Schweizerischer Bundesrat 1962, 897) mitinbegriffen sei, andererseits aber auch stärker die berufliche Weiterbildung und die Höheren Fachschulen und Technikerschulen unter diese Bestimmungen fallen. Hiermit wurde auch der staatsbürgerlichen und der industriellen Konvention Rechnung getragen, indem die bedeutsame Rolle der Berufsbildung für die Staaatsbürgerschaft wie auch die Möglichkeit, sich in der industriellen Arbeitswelt weiterzuentwickeln, gesetzlich hervorgehoben wurde.

Die Botschaft zum Berufsbildungsgesetz von 1978 zieht einleitend eine positive Bilanz der vorgängigen Berufsbildungsgesetzgebung, die sich ,offensichtlich bewährt" habe, da sie wesentlich zur Entwicklung und zum Ausbau der Berufsbildung beigetragen habe. Die Verankerung von allgemein 
gehaltenen Grundsätzen habe „entwicklungsoffenen Regelungen“ Raum belassen (vgl. Schweizerischer Bundesrat 1977, 684). Dennoch ergäbe sich weiterer Reformbedarf, was daraus ersichtlich werde, dass sich Verbände der Arbeitgeberinnen und Arbeitgeber, der Arbeitnehmerinnen und Arbeitnehmer wie auch Lehrerinnen- und Lehrerverbände der beruflichen Schulen mit Reformpostulaten zu Wort gemeldet hätten (vgl. ebd., 690f.). In der gleichen Botschaft wurde nun erstmals auch die Berufsbildung als „Teil des Bildungswesens“ hervorgehoben, auch wenn diese ,anders geartet" sei. Die Berufsbildung sei - so wird bekräftigt - „ein wesentlicher Bestandteil des Bildungswesens" und keine Sackgasse, sondern Ausgangspunkt breiter und vielfältiger beruflicher Tätigkeiten und Ausgangspunkt für fähige und bildungswillige Absolventinnen und Absolventen für den tertiären Bildungsbereich (vgl. ebd., 693f.).

\subsection{Zum Charakter der Berufsbildung als Rahmengesetzgebung}

Wie in vielen anderen Bildungsbereichen hat im Verlaufe des 20. Jahrhunderts eine weitere Formalisierung und Regulierung eines früher informellen Ausbildungsverhältnisses stattgefunden. Handwerkliche Qualitätsansprüche, industrielle Weiterbildungsmöglichkeiten, berufliche Regelungen, welche die Marktsposition stärkten und Schutzklauseln, die den Lehrling fern von Ausbeutung als gesellschaftlich-staatsbürgerliches aber auch als charakterlich erziehbares Wesen betrachteten, fanden Eingang in die Berufsbildungsgesetzgebung, bzw. wurden laufend gestärkt.

Die in der Folge ab 1930 etablierten gesamtschweizerischen Berufsbildungsgesetze waren und sind als Fördergesetzgebungen konzipiert und beruhen auf einer „Einverständnisgemeinschaft“ (vgl. Weber 1985, 417). Hier bieten sich Rahmengesetze an, die eine Vielzahl von Qualitätsansprüchen integrieren und unterschiedliche Konventionen bündeln. Sie lassen eine Vielzahl an Umsetzungs- und Kombinationsvarianten zu. Die Legitimität verdankt sich nicht einem „Systempaternalismus“ sondern vor allem den Verfahren und ihren institutionalisierten Begründungsforderungen (vgl. Habermas 1992, 563). Die Akteure können hierbei von einer hohen Zustimmungserwartung ausgehen, da auf vorhandene Motive zurückgegriffen wird (vgl. Luhmann 2010).

Gesetze klären nicht nur Zuständigkeiten beziehungsweise Kompetenzen, sondern sie eröffnen auch Möglichkeiten. Diese Ermöglichungsfunktion ist im Besonderen in Rahmengesetzgebungen gewährleistet. Auch um den Preis von „Wertungsinkongruenzen“ (vgl. Richli 2008, 148) bietet ein Rahmengesetz Spielräume für Aushandlungsprozesse und partielle Konfliktvermeidung. Die in weiteren nachgeordneten Gesetzen und Verordnungen festgeschriebenen Ergebnisse sind Hybride, die unterschiedliche und zum Teil 
auch gegensätzliche Anliegen integrieren. So ermöglicht die Berufsmaturität (siehe weiter unten) einen berufsqualifizierenden Abschluss und gleichzeitig eine akademische Studienberechtigung.

\subsection{Horizontale und vertikale Hybridbildungen}

Hybride kombinieren in einem Sowohl-Als-Auch verschiedene Konventionen und Qualitätsansprüche. Wir können im Zusammenhang mit der Entwicklung der Berufsbildung eine horizontale und eine vertikale Hybridisierung unterscheiden. Persönlichkeitsbildung und berufliche Qualifizierung, wie sie mit der Berufsbildungsförderungsgesetzgebung in der Schweiz entstanden sind, kennzeichnen eine horizontale Hybridisierung, die gleichzeitig verschiedene Qualitätsansprüche wie Wettbewerbsfähigkeit, Effizienz, Tüchtigkeit und gesellschaftlich-staatsbürgerliche Ansprüche integriert. Auch eine vertikale Hybridisierung verknüpft Qualitätsansprüche, die berufliche Bildung ist hier jedoch stärker auf eine zeitliche Sequenzierung ausgerichtet, indem Arbeitsmarktbefähigung mit einer Zugangsberechtigung zum Hochschulsystem kombiniert oder im Nachhinein erworben wird. Während die horizontale Hybridbildung zwei Wertigkeiten zu einer neuen Einheit und Qualität integriert, lässt die vertikale diese parallel als Sowohl-als-Auch bestehen.

Als Beitrag zur horizontalen Hybridisierung ist die Entwicklung der schweizerischen Berufsbildungsgesetzgebung zu sehen. So wird im neuesten Berufsbildungsgesetz (2002) festgehalten, dass dieses „die berufliche und persönliche Entfaltung und die Integration in die Gesellschaft, insbesondere in die Arbeitswelt" der oder des Einzelnen ermögliche und die Fähigkeit und die Bereitschaft vermittle, „beruflich flexibel zu sein und in der Arbeitswelt zu bestehen“; weiter, dass es „der Wettbewerbsfähigkeit der Betriebe“ diene und außerdem ,den Ausgleich der Bildungschancen in sozialer und regionaler Hinsicht, die tatsächliche Gleichstellung von Frau und Mann sowie die Beseitigung von Benachteiligungen von Menschen mit Behinderungen“" ermögliche (vgl. BBG 2002, Art. 3 a-c). Eher der vertikalen Hybridisierung dienlich ist die nachfolgende Bestimmung, dass ,die Durchlässigkeit zwischen verschiedenen Bildungsgängen und -formen innerhalb der Berufsbildung sowie zwischen der Berufsbildung und den übrigen Bildungsbereichen“" zu fördern sei (vgl. BBG 2002, Art. 3 d). 


\section{Die schweizerische Berufsmaturität als vertikale Hybridbildung der Berufs- und Allgemeinbildung}

Wie bereits festgehalten führten die seit der ersten Berufsbildungsgesetzgebung laufenden Aushandlungsprozesse zu einer horizontalen Hybridisierung der Berufsbildung, die insbesondere staatsbürgerliche, marktbezogene und industrielle Konventionen integrierte. In den 1990er Jahren ergab sich nun eine zweite Form der Hybridisierung: die Berufsmaturität.

\subsection{Die Berufsmaturität als Kompromiss}

Im Folgenden soll dieser Prozess der Enstehung der Berufsmaturität näher beleuchtet werden. Dabei steht die technische Berufsmaturität im Vordergrund, da sie als erste Richtung eingeführt wurde und somit auch als eine Art Beispiel für die später folgenden Berufsmaturitätsrichtungen stand. Ein spezifisches Augenmerk soll hierbei auf die Position(en) der Berufs- und Branchenverbände gelegt werden.

Die Einführung der Berufsmaturität anfangs der 1990er Jahre reiht sich als weiterer Kompromiss staatsbürgerlicher, marktbezogener und industrieller Konventionen in die Geschichte der schweizerischen Berufsbildung ein. Als zentrale Akteure dieses Aushandlungsprozesses lassen sich auf der einen Seite die Konferenzen der Rektorinnen und Rektoren und jene der Lehrkräfte der damaligen Berufsmittelschulen ausmachen und auf der anderen Seite die Vertreterinnen und Vertreter der Höheren Technischen Lehranstalten (HTL). Die Rektoren- und Lehrkräftekonferenzen der Berufsmittelschulen - welche bereits seit Ende der 1960er Jahren ergänzende und erweiterte Allgemeinbildung vermittelten - setzten sich über mehrere Jahre für eine verbesserte Anerkennung der Berufsmittelschul-Abschlüsse ein. Ihr Ziel war die Schweiz weit prüfungsfreie Zulassung zu den Höheren Lehranstalten für Technik und Wirtschaft (HTL \& HWV) (vgl. Kiener \& Gonon 1998). Diese Forderungen bezogen sich auf die sehr unterschiedlichen Zulassungsbedingungen der Höheren Technischen Lehranstalten und der damit verbundenen Ungewissheit über den Mehrwert der erweiterten Allgemeinbildung wie sie von den Berufsmittelschulen seit Jahrzehnten angeboten wurde (vgl. Ochsenbein 1991). Die Stärkung der Durchlässigkeit von der beruflichen Grundbildung in weiterführende Studiengänge der Tertiärstufe stand damit im Vordergrund. Nicht zuletzt wurde mit diesen Forderungen aber auch eine Attraktivitässteigerung der beruflichen Grundbildung angestrebt um der Abwanderung begabter Schülerinnen und Schüler in die Gymnasien Einhalt zu gebieten. Auf der anderen Seite forderten insbesondere die Höheren Technischen Lehran- 
stalten eine deutlich breitere Allgemeinbildung und mehr Theoriewissen ihrer Bewerberinnen und Bewerber als sie die Berufsmittelschulen bisher anboten. Auf der einen Seite sollte das Ausbildungsniveau den erweiterten Anforderungen angepasst und gleichzeitig weiterhin hochgehalten werden können. Doch nicht nur zusätzliche inhaltliche Ansprüche und damit auch die Erhaltung einer hohen Qualität der Ausbildung wurden geltend gemacht, auch spielte auf der anderen Seite die Forderung nach einer Aufwertung der Position der Höheren Lehranstalten innerhalb des Bildungssystems als Ganzes eine immer prägnantere Rolle. Die Forderung lautete, dass sie neu als Fachhochschulen auf Tertiärstufe, das heißt also auf gleicher Ebene wie die universitären Hochschulen, figurieren sollen. Vor allem die Mobilität und Anerkennung schweizerischer Fachkräfte im Ausland sollte so gestärkt werden (vgl. Gonon 1998, 2013).

Man war sich also grundsätzlich einig, dass die Vorbildung zu den Höheren Technischen Lehranstalten einer Aufwertung bedurfte. Sei es aus Sicht der Berufsmittelschulen mit Bezug auf die Möglichkeit eines beruflichgesellschaftlichen Aufstiegs durch eine allgemeine Verbesserung der Durchlässigkeit und der Schaffung eines Mehrwerts des BerufsmittelschulAbschlusses. Oder aus Sicht der Höheren Technischen Lehranstalten mit dem Bezug auf Wettbewerbsfähigket im Sinne einer Qualitätssteigerung ihrer Bildungsabschlüsse. Für diese Qualitätsansprüche ergab es in den 1990er Jahren ein „günstiges politisches Umfeld“ (vgl. Kiener \& Gonon 1998, 50), das sich unter anderem auch darin zeigte, dass mit einem Westschweizer Bundesrat eine Person das entsprechende Dossier führte, die sich hinsichtlich schulisch organisierter Berufsbildung und Stärkung des allgemeinbildenden Anteils in der Berufsbildung sehr affin zeigte. Die Ausgestaltung dieser erweiterten Allgemeinbildung bezüglich Inhalt und Umfang war in der Folge Gegenstand weiterer Aushandlungsprozesse. An diesem Punkt bringen denn auch die Berufs- und Branchenverbände ihre Forderungen in die Diskussion. Diese sollen nun im folgenden Abschnitt vertiefter betrachtet werden.

\subsection{Berufs- und Branchenverbände als Gestaltungsakteure der Berufsmaturität}

Der anhaltend stark beklagte Fachkräftemangel lässt die Vermutung zu, dass die Beruf und Branchen vertretenden Akteure ein starkes Interesse an strukturierten und zielführenden Übergängen von der Berufsbildung in weiterführende Schulen hatten. Die entsprechenden Verbände spielten bei der Einführung der technischen Berufsmaturität jedoch auf den ersten Blick nur eine sehr marginale Rolle, bzw. hatten sich erst im Rahmen der offiziellen Vernehmlassung zu den Verordnungsänderungen eingeklinkt (siehe dazu: Kiener \& Gonon 1998). Tatsächlich traten die Verbände bei den Vorbereitungen zur 
Einführung der technischen Berufsmaturität, welche eine Art Vorreiterrolle hinsichtlich der heutigen Abschlüsse in sechs Fachrichtungen spielte, nicht direkt in Erscheinung. Ein intensiver Austausch mit den damaligen Höheren Technischen Lehranstalten bzw. Ingenieurschulen, welche aktiv an der Erarbeitung der technischen Berufsmaturität mitarbeiteten, ist jedoch nicht von der Hand $\mathrm{zu}$ weisen. So wehrten sich vor allem kleinere Unternehmen und Arbeitgeberverbände über Jahre vehement und erfolgreich gegen den Vorschlag der Direktorenkonferenz der Ingenieurschulen der Schweiz (DIS), die praktische Lehrzeit für angehende Studierende Höherer Technischer Lehranstalten um ein Jahr zugunsten einer erweiterten Allgemeinbildung zu verkürzen. Denn gerade dieses vierte und letzte Lehrjahr spielte für die Betriebe hinsichtlich Rentabilitätsüberlegungen eine zentrale Rolle (vgl. Bähler 1990; Zellweger 1991). Der Qualitätsanspruch der Wettbewerbsfähigkeit und damit die marktliche Konvention stehen hiermit im Vordergrund dieser Argumentation. Eine stärkere Gewichtung der europäischen Perspektive ergab sich auch aus den bestehenden Austauschkanälen, die zu einer engeren Zusammenarbeit zwischen den Akteuren führte. So zeichneten für die Durchführung eines nationalen ,HTL-Tages' sowohl der Branchenverband STV (Schweizerischer Technischer Verband) wie auch die Direktorenkonferenz der Ingenieurschulen der Schweiz (DIS) verantwortlich. Das hochkarätig besetzte Teilnehmerfeld dieser Tagung versuchte auszuloten welche Wege zu einer internationalen Anerkennung der HTL-Diplome als zielführend erachtet werden konnten (vgl. Jahresbericht STV 1990). Die Thematik der Positionierung der HTLStudiengänge war - wie daraus ersichtlich wird - ein dringliches Anliegen der bildungspolitischen Bestrebungen des Arbeitgeberverbandes Schweizer Maschinen- und Metall-Industrieller (ASM) und des Schweizerischen Technischen Verbandes (STV), den Ende der 80er und Anfangs 90er Jahren zwei zentralen Verbände der Technikbranche. Der Fokus lag jedoch klar auf internationaler Anerkennung. So heißt es im Jahresbericht des Schweizerischen Technischen Verbandes (STV) des Jahres 1989: „Während uns in den letzten Jahrzehnten die Anerkennung des Ingenieurs in der schweizerischen Gesellschaft stark beschäftigte, fragen wir uns heute: Wo steht der Ingenieur HTL in Europa?" (S. 4). Wenn man sich die Position der technischen Branchenverbände genauer anschaut, fällt auf, dass sie sich sehr stark für die Anerkennung der HTL-Abschlüsse und Titel im Europäischen Bildungsraum eingesetzt haben. Das Ziel war, dass Inhaberinnen und Inhaber eines HTLAbschlusses auch den Titel ,EUR ING' der ,European Federation of National Engineering Associations' (ein europäischer Zusammenschluss nationaler Branchenverbände der Ingenieure - kurz FEANI) führen dürften. Die technischen Berufs- und Branchenverbände setzten sich zu Beginn also stärker für 
eine Aushandlung im internationalen Kontext ein als für eine bildungspolitische Lösung im nationalen Kontext. Mit dieser internationalen Orientierung und Anerkennung sollte indirekt auch die nationale Position der HTL gestärkt werden.. Dies primär gegenüber der Eidgenössisch Technischen Hochschule, welche ebenfalls Ingenierinnen, Ingenieure, Architektinnen und Architekten ausbildete, allerdings auf Hochschulebene. Die Verknüpfung der Verbandslösung im internationalen Kontext mit der nationalen bildungspolitischen Lösung lag jedoch eigentlich auf der Hand, denn ,FEANI' war der Ansicht, „dass einzig eine Matura relevante Zulassungsvoraussetzung zu einem echten Hochschulstudium“ (vgl. STV 1990, 5) sei. Eine nicht vorhandene Maturität verunmöglichte folglich eine entsprechende Anerkennung der HTL-Studiengänge. Für Hans Jörg Bodenmann, damaliger Zentralpräsident des STV, war denn auch klar: „Hätten unsere Parlamentarier den Mut gehabt, den Gedanken der Berufsmittelschule zu Ende zu denken, d.h. mit einer Berufsmaturität abzuschließen, wäre ein wesentliches Hindernis ausgeräumt" (vgl. STV 1989, 4). Einer Anerkennung durch FEANI standen also die bestehenden Zulassungsbestimmungen zu den Höheren Lehranstalten im Wege. Die Schaffung einer technischen Berufsmaturität wurde daher in der Folge zu einem wichtigen Schwerpunkt im Zusammenhang mit der Verbandspolitik zum Thema „EURO-Ingenieur“ und der „HTL-Reform 92“ (vgl. STV 1991).

Im Bezug auf die zu Beginn dieses Beitrages dargelegten Konventionen wird deutlich, dass für die Berufs- und Branchenverbände die marktbezogene Konvention und der Qualitätsanspruch der Mobilität und Wettbewerbsfähigkeit der ausgebildeten technischen Fachkräfte von zentraler Bedeutung war. Die staatsbürgerliche Konvention, welche das Gerechtigkeits-Argument ins Feld führt, beziehungsweise rechtliche Gleichbehandlung der 3-jährigen Vollzeitstudiengänge der damaligen Höheren Fachschulen HTL und HWV mit anderen in ihrer Länge vergleichbaren Studiengängen der Tertiärstufe ins Zentrum rückte, drängte sich erst mit Beginn der 90er Jahre an die Seite der marktlichen Konvention. Am Rand wird auch eine Relevanz der industriellen Konvention sichtbar, welche die mit der Einführung der Berufsmaturität einhergehende Aufwertung der berufsbildungsbasierten Ingenieurausbildung beleuchtet und damit die Funktionalität beziehungsweise Effizienz des berufliche Bildungssystems betont.

In dem hier Bezüge auf situationsbestimmende Konventionen sichtbar gemacht werden, wird uns Einsicht in Prozesse der Handlungskoordination und Entscheidungsfindung geboten, die sonst kaum beachtet worden wären. Es sei jedoch darauf hingewiesen, dass primär die Position der Berufs- und Branchenverbände herausgearbeitet wurde. Für die Darstellung der Gesamtprozessentwicklung (und damit auch der zu Beginn definierten Konventionen 
und Kompromissbildungen) müssten jedoch noch weitere Akteure und Positionen genauer analysiert werden.

Abschließend kann festgehalten werden, dass sich die Berufsmaturität als bedeutsame Innovation und zentrales Element der schweizerischen Berufsbildung etabliert hat. Ein zentraler Kompromiss zeigt sich beispielsweise im Bezug auf den Umfang der erweiterten Allgemeinbildung: die Lehrzeit als Ganzes wurde nicht gekürzt (was dem Wunsch der Höheren Lehranstalten entsprochen hätte), im Gegenzug kann aber die praktische Ausbildung im Lehrbetrieb auf bis zu drei Tage pro Woche reduziert werden - was der größtmöglichen Berücksichtigung der Rentabilitätsüberlegungen von Lehrbetrieben entspricht. Die heute bestehenden sechs Fachrichtungen (technisch, kaufmännisch, gestalterisch, gewerblich, gesundheitlich \& sozial, naturwissenschaftlich) stellen ein weiteres wesentliches Merkmal dar, für das sich insbesondere die Branchenverbände aber auch Akteure aus dem Kreis der heutigen Fachhochschulen stark gemacht hatten (vgl. EVD 2009). Empirische Daten zeigen denn auch, dass die Passung zwischen Berufsmaturitätsund anschliessend gewählter Studienrichtung einen wesentlichen Einfluss auf den Studienerfolg hat. So sind die Berufsmaturitäts-Absolventinnen und absolventen mit der für den Fachhochschul-Fachbereich passenden Berufsmaturität ,alle überdurchschnittlich erfolgreich“ (vgl. Kaiser, Grütter \& Fitzli 2013, 64).

\section{Schlussfolgerungen}

Die schweizerische Berufsbildung und ihre Entwicklung, sowie auch ihre Integration in das gesamte Bildungssystem, mit verhältnismäßig niederschwelligen Übergängen von der Berufsbildung in das Allgemeinbildungssystem sind ein Paradebeispiel, wie sich, Offe folgend, eine partikularismenreduzierende und optionenerweiternde Integration im Sinne eines SowohlAls-Auch unterschiedlicher Qualitätsansprüche vollzogen hat. Rahmengesetzgebungen haben sich hierbei als offene Gefäße entpuppt, die eine Entwicklung der Berufsbildung und eine doch erhebliche Modifikation des Berufsbildungssystems ermöglicht haben. Reale und mögliche Konflikte wurden durch situative Kompromisse pragmatisch prozessiert. Die Erweiterung von Spielräumen wurde durch eine stärkere Flexibilisierung und Durchlässigkeit des Berufsbildungsystems erreicht, welches darüber hinaus auch einen Hochschulzugang gewährte: durch eine Hybridisierung der Berufsbildung.

Der konventionssoziologische Ansatz sollte in diesem Zusammenhang eine veränderte Perspektive auf Akteure und ihre Koordinationsprozesse und die Erweiterung von Möglichkeitsräumen sichtbar machen. Diese Ausarbeitung von Akteurspositionen und ihr Umgang mit Institutionen ist jedoch 
hinsichtlich der Bestimmung von Konventionen mit einer Interpretationsproblematik konfrontiert, die Zuordnungen von Qualitätsansprüchen erschweren. Des Weiteren ist offen, ob die eingeschränkte Wahl der hier entfalteten Kategorien sich auch in der Folge bewährt und wie mit dem multiplen Vorhandensein differenter Konventionen umzugehen ist.

\section{Quellen}

Bundesgesetz über die Berufsbildung (BBG) vom 13. Dezember 2002 (SR 412.10) [Einsehbar im Bundesblatt, 154, Seiten 4557-4586 oder online: https://www. admin.ch/opc/de/classified-compilation/20001860/index.html, Stand 15.03.2016].

EVD (Eidgenössisches Volkswirtschaftsdepartement) (2009). Verordnung über die eidgenössische Berufsmaturität (Berufsmaturitätsverordnung). Bericht über die Ergebnisse der Vernehmlassung. Bern. Online: http://www.news.admin.ch/NSBSubscriber/message/attachments/16084.pdf.

Schweizerischer Bundesrat (1928). Botschaft des Bundesrates an die Bundesversammlung zum Entwurf eines Bundesgesetzes über die berufliche Ausbildung. Bundesblatt, 80, 725-798.

Schweizerischer Bundesrat (1962). Botschaft des Bundesrates an die Bundesversammlung zum Entwurf eines Bundesgesetzes über die Berufsbildung. Bundesblatt, 114, 885-980.

Schweizerischer Bundesrat (1977). Botschaft des Bundesrates an die Bundesversammlung betreffend Änderung des Bundesgesetzes über die Berufsbildung. Bundesblatt, 123, 1207-1216.

SGB (Schweizerischer Gewerkschaftsbund) (1912). Eingabe betreffend ein Eidgenössisches Lehrlingsgesetz. Bern

SGV (Schweizerischer Gewerbeverband) (1918). Entwurf eines Bundesgesetzes betreffend Berufslehre und Berufsbildung. In: SGV (Hrsg.): Gewerbliche Zeitfragen, Vorarbeiten des Schweizerischen Gewerbeverbandes für die Schweizerische Gewerbegesetzgebung, XXVIII, Bern: Verlag des Schweizerischen Gewerbeverbandes.

STV (Schweizerischer Technischer Verband) (1989). Jahresbericht 1989. Zürich: STV.

STV (Schweizerischer Technischer Verband) (1990). Jahresbericht 1990. Zürich: STV.

STV (Schweizerischer Technischer Verband) (1991). Jahresbericht 1991. Zürich: STV. 


\section{Literatur}

Bähler, R. (1990). Wer fürchtet sich vor der Fachhochschule? Katapult, 11, o.S.

Berner, E., Gonon, Ph. \& Ritter, H-J. (2011). Zwischen Gewerbeförderung, Sozialpolitik und liberalen Bildungsbestrebungen - Zur 'Vor'-Geschichte der dualen Berufsbildung in der Schweiz. Zeitschrift für Berufs- und Wirtschaftspädagogik, 107 (1), 14-32.

Bernet, F. (1923). Lehrlingsausbildung und Lehrlingsfürsorge in einigen Großbetrieben der schweizerischen Metall- und Maschinenindustrie. Zürich: Orell Füssli.

Boltanski, L. \& Thévenot, L. (2007). Über die Rechtfertigung. Eine Soziologie der kritischen Urteilskraft. Hamburg: Hamburger Edition.

Busemeyer, M. (2009). Wandel trotz Reformstau. Die Politik der beruflichen Bildung seit 1970. Frankfurt: Campus.

Diaz-Bone, R. (2011). Einführung in die Soziologie der Konventionen. In R. DiazBone (Hrsg.), Soziologie der Konventionen. Grundlagen einer pragmatischen Anthropologie (S. 9-42). Frankfurt: Campus.

Gonon, P. (1998). Genese und „Wirksamkeit“ der technischen Berufsmaturität im Spannungsfeld bildungspolitischer Interessenaushandlung. In A. Schelten, P.F.E. Sloane \& G.A. Straka (Hrsg.), Perspektiven des Lernens in der Berufsbildung. Forschungsberichte der Frühjahrstagung 1997 (S. 37-55). Opladen: Leske + Budrich.

Gonon, P. (2013). Berufsmaturität als Reform - Hybris oder Erfolgstory? In M. Maurer \& P. Gonon (Hrsg.), Herausforderungen für die Berufsbildung in der Schweiz. Bestandesaufnahme und Perspektiven, (S. 119-145). Bern: hep.

Habermas, J. (1992). Faktizität und Geltung. Beiträge zur Diskurstheorie des Rechts und des demokratischen Rechtsstaats. Frankfurt: Suhrkamp.

Imdorf, Ch., Berner, E \& Gonon, P. (2015). Duale versus vollzeitschulische Berufsausbildung in der Schweiz - Zwei Institutionalsierungsmuster der beruflichen Bidlung aus rechtfertigungstheoretischer und kanontal vergleichender Perspektive. In R. Leemann, Ch. Imdorf, J. Powell, \& M. Sertl, Die Organisation von Bildung (S.186-207). Weinheim \& Basel: Beltz Juventa.

Kaiser, N., Grütter, M. \& Fitzli, D. (2013). Evaluation der Studierfähigkeit von Berufsmaturitäts-Absolventen/innen an den Fachhochschulen. Analyse der Statistischen Daten. Bericht zum Teilprojekt 1. Zürich: econcept.

Kiener, U. \& Gonon, P. (1998). Die Berufsmatur. Ein Fallbeispiel schweizerischer Berufsbildungspolitik. Chur/Zürich: Rüegger.

Luhmann, N. (2010). Politische Soziologie. Frankfurt: Suhrkamp.

Neu, T. (2015). Koordination und Kalkül. Die Économie des conventions und die Geschichtswissenschaft. Historische Anthropologie, 68(1), 129-147.

Ochsenbein, H. (1991). Zum Übertritt von der gewerblich-industriellen Berufsbildung an die Ingenieurschulen. Schweizerische Blätter für beruflichen Unterricht, 5, 206-209.

Offe, C. (1971). Berufsbildungsreform. Eine Fallstudie über Reformpolitik. Frankfurt: Suhrkamp. 
Richli, P. (2008). Berufsbildungsrecht. Auf dem Weg zum gleichberechtigten Spross des Bildungsrechts. In T. Bauder \& F. Osterwalder (Hrsg.), 75 Jahre eidgenössisches Berufsbildungsgesetz. Politische, pädagogische, ökonomische Perspektiven (S. 129-152). Bern: hep.

Weber, M. (1985). Wirtschaft und Gesellschaft. Grundriss der verstehenden Soziologie. 5. Aufl. Tübingen: Mohr.

Zellweger, M. (1991). Es bewegt sich. Die Ingenieurausbildung in der Schweiz in einem Europa von morgen. Technische Rundschau, 45, 36-41. 


\title{
Berufslaufbahnkonzepte: Instrumente zur Weiterentwicklung des Berufsbildungssystems?
}

\author{
Rolf Dobischat, Andy Schäfer, Christian Schmidt, Manfred Wahle, Marcel \\ Walter
}

\section{Abstract}

Angefangen mit den „Berufebaukästen“ des Zentralverbands des Deutschen Handwerks wurden in den letzten Jahren regelmäßig sog. Berufslaufbahnkonzepte in die Fachdiskussion eingebracht und ihre Praktikabilität sowie flächendeckende Umsetzbarkeit im Rahmen von Modellversuchen eruiert. Berufslaufbahnkonzepte zielen zum einen darauf ab, das Berufsbildungssystem für sämtliche darin agierende Akteure transparenter zu gestalten und bilden zum anderen eine Grundlage für berufsbildungspolitische Reformen. In dem vorliegenden Beitrag werden die wesentlichen Entstehungsbedingungen aufgezeigt, die für die Formulierung von Berufslaufbahnkonzepten verantwortlich sind. Davon ausgehend werden die Kernelemente bestehender Laufbahnkonzepte zu einem idealtypischen Laufbahnmodell verdichtet und auf dieser Grundlage die Funktionen von Laufbahnkonzepten diskutiert. Ihre Eigenschaft, berufsbildungspolitische Reformdebatten aufzugreifen und auf die Mobilität im Berufsbildungssystem zuzuspitzen, ist zugleich Hauptstärke und Hauptschwäche der Konzepte. Der letzte Abschnitt beschließt den Beitrag daher mit einem Verweis auf Probleme und Perspektiven.

\section{Einleitung}

Das Duale System der Berufsausbildung gerät seit einigen Jahren von zwei Seiten unter Druck. Am unteren Rand besteht die Herausforderung darin, bildungsschwächere Jugendliche zu integrieren und zu einem erfolgreichen Ausbildungsabschluss zu führen (vgl. Schmidt 2012). Der demografische Wandel führt dazu, dass die beschriebene Bedrohung „,von unten“ zwar abnimmt, stellt die Betriebe jedoch künftig zunehmend vor das Problem, ausreichend Fachkräftenachwuchs zu rekrutieren.

Gleichzeitig ist die duale Ausbildung im Vergleich zum Hochschulstudium für leistungsstärkere Jugendliche immer weniger attraktiv. Im Vergleich zur Hochschulbildung vermag sie es kaum, attraktive Karriereoptionen in 
Aussicht zu stellen. Lange Zeit war dies auch nicht ihre Aufgabe, kam doch ein Übergang vom quantitativ erheblich stärker besetzten Bereich der betrieblich-beruflichen Qualifizierung in das quantitativ eher schwach besetzte Segment der Hochschulqualifizierung nur für einen Bruchteil der Individuen in Frage.

Unter der Prämisse, dass die Wirtschaft ihren Nachwuchs auch künftig zu großen Teilen über die duale Ausbildung rekrutieren möchte und die duale Ausbildung auch weiterhin als zentrale Triebfeder der gesellschaftlichen Integration Jugendlicher dienen soll, erscheinen allerdings Maßnahmen notwendig, um die Attraktivität des Dualen Systems zu fördern. An diesem Punkt setzen Berufslaufbahnkonzepte an. Sie skizzieren zwar Bildungswege von der vorberuflichen Bildung bis in den Hochschulbereich, allerdings steht die duale Ausbildung als Ziel der vorberuflichen Bildung und als Grundlage für alle weiteren Bildungsentscheidungen deutlich im Zentrum aller Ansätze.

Erste Ansätze zu beruflichen Laufbahnkonzepten kommen maßgeblich vom Zentralverband des Deutschen Handwerks (ZDH) (vgl. ZDH 2004; Esser 2003; ZDH 2007; Heinsberg \& Rehbold 2011; Born 2012). Wissenschaftliche Beiträge, die das Thema beruflicher Laufbahnen auf der MetaEbene, insbesondere mit Fokus auf den Übergang zum Hochschulbereich diskutieren, stammen aus dem ITB (vgl. Rauner 2012; Gerds \& Spöttl 2010) sowie von Becker (2011) und Euler (im Ersch.).

Der ZDH suchte mit seinem Konzept einen Mittelweg zwischen Öffnung und Flexibilisierung des Berufsbildungssystems und Erhalt des ganzheitlichberuflichen Charakters dualer Ausbildung. Das ursprüngliche Konzept des $\mathrm{ZDH}$ sah vor, Bildungs- und Beschäftigungswege in einem bzw. mehreren artverwandten Berufen zu skizzieren und orientierte sich an den bestehenden formalisierten Aus- und Weiterbildungsangeboten. Es unterschied dabei die drei Qualifizierungsstufen Ausbildungs-, Gesellen- und Hochschulebene (Meister-Ebene und sog. Meister-Plus-Ebene) und ordnete diesen Stufen jeweils alle formalen Aus- und Fortbildungsmöglichkeiten einer Domäne zu. Heinsberg \& Rehbold (2011) haben das Konzept auf die Gesundheitshandwerke übertragen und weiterentwickelt. Dabei wurde es um eine vorberufliche Ebene ergänzt (Berufsvorbereitung) und es wurden der Meister-PlusEbene auch Studiengänge und die Promotion hinzugefügt. Weiterhin wurde das Modell für non-formale Qualifizierungen geöffnet, sodass es insgesamt deutlich an Umfang gewann. Schließlich erfolgte eine stärkere Zuordnung der Qualifizierungsebenen zu den DQR-Niveaustufen.

Da Berufslaufbahnkonzepte darauf abzielen, den Kern- und Ankerpunkt der Berufs- und Wirtschaftspädagogik - das Duale System - abzusichern, empfehlen sie sich als berufs- und wirtschaftspädagogisches Forschungsfeld. Die dem vorliegenden Beitrag zugrundeliegende übergeordnete Fragestellung lautet: Worin besteht der gemeinsame Kern von bisher in die berufsbildungspolitische Diskussion eingebrachten Berufslaufbahnkonzeptionen und in- 
wieweit leisten Berufslaufbahnkonzepte einen Beitrag zur attraktivitätssteigernden Weiterentwicklung beruflicher Bildung vor dem Hintergrund struktureller Veränderungen? Zur Beantwortung beschreiben wir zunächst die strukturellen Ursachen, die zur Entwicklung von Laufbahnkonzepten geführt haben (Kapitel 2). Daraufhin stellen wir die zentralen Funktionen von Laufbahnkonzepten dar und diskutieren den konkreten Aufbau solcher Konzepte anhand eines idealtypischen Modells, das den Kern aller bisher in die Diskussion eingebrachten Laufbahnkonzepte bildet (Kapitel 3). Der Beitrag schließt mit einer kritischen Auseinandersetzung bzgl. des Umsetzungs- und Reformpotenzials von Berufslaufbahnkonzepten (Kapitel 4). Die theoretischen und empirischen Ausgangs- bzw. Anknüpfungspunkte zur Beantwortung der o.g. Fragestellung sind zum einen die verschiedenen Aspekte des sozialen Wandels (Tertiarisierung, Höherqualifizierungstendenzen, demografische Entwicklung und Europäisierung) und zum anderen Überlegungen zur verstärkten Öffnung des Bildungssystems (Durchlässigkeit und Mobilität).

\section{Sozialer Wandel: Ausgangspunkte für die Formulierung von Berufslaufbahnkonzepten}

\subsection{Strukturwandel/Wissensgesellschaft und Lebenslanges Lernen}

Der Strukturwandel hin zu einer Dienstleistungsgesellschaft, in der nicht nur immer mehr Menschen im Dienstleistungsbereich arbeiten, sondern sich auch Arbeit im industriellen Sektor zunehmend „tertiarisiert“, wird unter dem Begriff „Wissensgesellschaft“ interpretiert als eine Umbruchssituation, in der wissensintensive Arbeitsanforderungen an Bedeutung gewinnen. Sowohl in Produktionsberufen als auch in sekundären Dienstleistungsberufen werden eine steigende Komplexität der Arbeitsinhalte und -anforderungen beobachtet (vgl. Walter 2014, 70f.).

Diese Entwicklungen begründen wiederum unter dem Leitbegriff des Lebenslangen Lernens eine Flexibilisierung beruflicher Aus- und Weiterbildungsprozesse. Lebenslanges Lernen als Bildungsprogrammatik kann hierbei durch zwei typische Charakteristika gekennzeichnet werden: Erstens wird die Vorstellung einer rigiden Trennung beruflicher Erstausbildung und anschlieBender beruflicher Weiterbildung in Frage gestellt. Strukturwandel und Wissensgesellschaft nötigten das Individuum zweitens zu einem lebenslangen Prozess der Aufrechterhaltung der Beschäftigungsfähigkeit durch permanente Qualifizierung (vgl. Maier \& Vogel 2013, 12).

Das Konzept des Lebenslangen Lernens ist damit höchst ambivalent: Dem idealisierten Bild der Individuen, die flexibel auf die Unwägbarkeiten 
einer immer schwerer planbaren Karriere reagieren sollen, stehen neue Zwänge, Unsicherheiten und die vielfachen Beschränkungen einer selbstbestimmten Gestaltung der beruflichen Karriere gegenüber. Berufliche Laufbahnen müssen sich schneller ändernden Qualifikationsanforderungen anpassen, Individuen stehen vor der Aufgabe, für den Erwerb und die Nutzung ihrer Kompetenzen in immer ausdifferenzierteren Lernumwelten mehr Verantwortung zu übernehmen (vgl. Pongratz \& Voß 2000). Die je nach Berufsfeldern, Betriebsgrößen und Branchen differenten betrieblichen Voraussetzungen und die den individuellen Wünschen häufig widersprechenden unternehmerischen Kalküle hinsichtlich der Ausgestaltung von Karriereoptionen und Wissensmanagement bedingen unterschiedliche Karrierepfade und legen in unterschiedlicher Weise Weiterbildungsaktivitäten nahe (vgl. Dobischat \& Schurgatz 2015).

Berufslaufbahnkonzepte setzen bei diesen institutionellen Gegebenheiten des Lebenslangen Lernens an. Sie sollen zum einen ermöglichen, was dem Individuum programmatisch abverlangt wird. Denn erst die Annahme, dass eine erste Ausbildung lediglich die Ausgangsbasis für notwendige weitere Qualifizierungsprozesse darstellt und horizontale wie vertikale Bewegungen der Individuen durch das Berufsbildungssystem auch bei Erwachsenen immer mehr die - gewünschte wie erforderliche - Regel als die Ausnahme darstellen (vgl. Lipsmeier \& Münk 1997), gibt Anlass dazu, ausgehend von der dualen Ausbildung über die vielfältigen Weiterentwicklungsmöglichkeiten zu informieren und Erweiterungen der Qualifizierungsoptionen zu eruieren. Zum anderen sollen sie Individuen dabei helfen, das enorm ausdifferenzierte Angebot an Qualifizierungsmöglichkeiten zu überblicken.

\subsection{Demografischer Wandel und Akademisierung}

Aktuell stellt ein spezifisches Zusammenspiel von demografischem Wandel und Akademisierung die berufliche Bildung vor besondere Herausforderungen. Nach Jahren eines deutlichen Bewerberüberhanges und einer großen Anzahl unversorgter Jugendlicher bewirkt der zahlenmäßige Rückgang der von den allgemeinbildenden Schulen abgehenden Kohorten einseitig eine Entlastung des Ausbildungsstellenmarktes. Das Ausbildungsstellenangebot stagniert, während die Nachfrage nach Ausbildung sinkt. Dieser Effekt wird durch einen ungebrochenen Trend hin zur Hochschulbildung und weg von der dualen Ausbildung unterstützt (vgl. Autorengruppe Bildungsberichterstattung 2014, 40; Baethge \& Wieck 2015, 4; Severing \& Teichler 2013, 9).

Neben der Frage veränderter Qualifikationsanforderungen rückt also auch jene der Attraktivität beruflicher Ausbildung unter den gegebenen Rahmenbedingungen in den Vordergrund. Junge Menschen schreiben der Hochschulbildung im Vergleich zur beruflichen Bildung eine höhere Ar- 
beitsplatzsicherheit, eine höhere Wahrscheinlichkeit eigenverantwortlichen Arbeitens und eigener Interessenverwirklichung sowie eine höhere berufliche Position und gesellschaftliche Anerkennung zu (vgl. Baethge, Kerst, Leszczensky \& Wieck 2014, 23f.). Die abnehmende Attraktivität der beruflichen Bildungsgänge korrespondiert mit der im Grundsatz meritokratischen Logik des Bildungssystems, die möglichst hohe formale Abschlüsse mit entsprechenden Bildungsrenditen und rates of return belohnt. An diesem Punkt setzen Berufslaufbahnkonzepte an: Sie zielen darauf ab, die duale Ausbildung durch ein Aufzeigen von Karrierewegen bis hin in den tertiären Bereich und eine Erleichterung der Übergänge zwischen den Qualifizierungsabschnitten attraktiver zu gestalten.

\subsection{Europäisierung}

Europäische Berufsbildungspolitik versucht seit jeher, die räumliche und berufliche Mobilität - und viel grundlegender: die Beschäftigungsfähigkeit der Erwerbstätigen zu erhöhen und damit Europa als Wirtschaftsstandort zu stärken. Hierzu gehört ganz besonders auch, die Durchlässigkeit innerhalb von und zwischen Bildungssystemen zu erhöhen, Durchlaufzeiten zu verkürzen, non-formal und informell erworbene Kompetenzen sichtbar zu machen und Anschlussmöglichkeiten im unteren (Übergangssystem) wie auch im oberen Segment (Tertiärbereich) auszuweiten. Weil sich die Nationalstaaten verbindlich dazu verpflichtet haben, Strategien zur Erreichung der europäischen Ziele im nationalen Kontext umzusetzen und den Grad der Umsetzung auch in Form von Benchmarks öffentlich zu machen und weil „europäische“ Ziele wie Mobilität, Durchlässigkeit im Bildungssystem, Outcome-Orientierung usw. zumindest in Teilen ohnehin Bestandteile jahrzehntelanger Reformbemühungen der Nationalstaaten sind, hat der europäische Diskurs einen erheblichen Einfluss auf die Diskussionen in den Nationalstaaten. Administrative Instrumente der EU, allen voran der Europäische Qualifikationsrahmen (EQR), bilden insofern aktuell die Reflektionsfläche, auf der sich sämtliche bildungspolitischen Reformbedarfe gebündelt diskutieren lassen (Münk 2008). Dies lässt sich auch am Beispiel der beruflichen Laufbahnkonzepte deutlich machen. Die europapolitischen Ziele wie Durchlässigkeit und Outcome-Orientierung, aber eben auch der Versuch, die europapolitischen Vorstellungen der Flexibilisierung von (Berufs-)Bildungssystemen mit dem deutschen Berufsprinzip zu vereinen, waren von Beginn an zentrale Leitmotive bei der Beschreibung und Forderung von Berufslaufbahnkonzepten (vgl. ZDH 2007; 2004). 


\section{Funktionen beruflicher Laufbahnkonzepte}

Berufliche Laufbahnkonzepte stellen Versuche dar, den sowohl von der Angebotsseite (veränderte Qualifikationsanforderungen, Europäisierung) als auch von der Nachfrageseite (demografischer Wandel und Akademisierung) bestehenden Anpassungsdruck auf das Berufsbildungssystem konstruktiv zu verarbeiten. Diese konstruktive Verarbeitung äußert sich auf zwei sehr unterschiedlichen Funktionsebenen: Zum einen dienen Berufslaufbahnkonzepte dazu, die Transparenz im Berufsbildungssystem für (potenzielle) Nachfrager/-innen und berufsbildungspolitische Akteure zu erhöhen. Zum anderen machen sie sich zur Aufgabe, Impulse für ganz konkrete ordnungspolitische Eingriffe in das Berufsbildungssystem zu liefern und das System so weiterzuentwickeln. Hierzu gehört sowohl die Schaffung oder Erweiterung von Durchlässigkeiten im Bildungssystem als auch - grundlegender - die aktive Auseinandersetzung mit den Zielen europäischer Berufsbildungspolitk (Mobilität, Beschäftigungsfähigkeit, Outcome-Orientierung etc.).

\subsection{Idealtypisches Laufbahnmodell}

Um diskutieren zu können, ob berufliche Laufbahnkonzepte dieses leisten können und um bei der näheren Beschreibung ihres Aufbaus von einzelnen sehr spezifischen Modellen zu abstrahieren, sind die zentralen Gestaltungselemente der bisher in die Diskussion eingebrachten Berufslaufbahnkonzepte nachstehend $\mathrm{zu}$ einem berufsübergreifenden idealtypischen Rahmenmodell verdichtet. Durch den engen Bezug zur Outcome-Orientierung, zur Durchlässigkeitsdebatte und zur Modularisierungsdiskussion sind einzelne Bildungsabschnitte in den Berufslaufbahnkonzepten als Qualifizierungsbausteine abgebildet, die von abgeschlossenen Qualifikationen (etwa duale Ausbildung) bis hin zu Teilqualifikationen (etwa Anpassungsfortbildung) jeden Grad an Formalisierung und Umfang annehmen können.

Grundsätzlich lassen sich eine horizontale und eine vertikale Anordnung der Qualifizierungsbausteine in Berufslaufbahnkonzepten unterscheiden. Die vertikale Achse bildet Qualifizierungsabschnitte bzw. -ebenen im Sinne von Niveaustufen ab, wobei einer Ebene durchaus mehrere gleichwertige Qualifikationen bzw. Qualifikationen mit Binnendifferenzierung innerhalb einer Ebene zugeordnet sein können. Die vertikale Anordnung orientiert sich dabei konsequent an den Niveaustufen des DQR. Ein zentraler Unterschied der Laufbahnkonzepte zum DQR ist dabei, dass den Niveaustufen in den Berufslaufbahnkonzepten bereits ganz selbstverständlich non-formale Qualifizierungsbausteine zugeordnet werden, während die offizielle DQR-Logik weiterhin formales Lernen fokussiert (vgl. BLK-DQR 2013). Der Zugang zur nächsthöheren Qualifikationsebene setzt in der Regel einen erfolgreichen 
Abschluss der darunterliegenden Ebene voraus, der im Rahmen einer formalen Prüfung sichergestellt wird.

Horizontal werden gleichwertige Aus- und Fortbildungsmöglichkeiten nebeneinander abgetragen. Hierbei erscheint eine deutliche Unterscheidung von formalen Qualifikationen, die den Standardweg markieren, und optionalen, auch non-formalen Zusatzqualifikationen sinnvoll. Hierdurch können berufstypische Laufbahnmuster, betriebliche Bedarfskombinationen und spezialisierte Tätigkeitsprofile abgebildet werden. Je nach Bedarf lassen sich die Qualifizierungsmöglichkeiten einer Ebene vollständig abbilden oder es erfolgt eine Konzentration auf ,typische“ bzw. besonders zentrale Qualifizierungen, wodurch Berufslaufbahnkonzepte auch als regional-spezfische Modelle denkbar werden. Die Modelle lassen sich horizontal auch um weitere Berufe erweitern, sodass sie Bezüge zu artverwandten Berufen (Berufsfamilie) abbilden. Für jede Qualifikation lassen sich auf einer zweiten Betrachtungsebene Informationen über die typische Dauer, Lerninhalte und Kompetenzziele und ggf. über die Gliederung in separat zertifizierbare Teilmodule/Bausteine sowie auf einer dritten Betrachtungsebene gar auf zugehörige Ordnungsmittel und Curricula verweisen. So wird von außen leichter ersichtlich, welche Inhalte und Anforderungsprofile mit spezifischen Karrieremustern jeweils verbunden sind.

Eine Verbindung der horizontalen und vertikalen Anordnung erfolgt über Pfadmodelle, mit denen sich berufstypische Karrierewege von der Berufsvorbereitung bis hin zum Tertiärbereich visualisieren lassen (etwa im Hörgeräteakustikerhandwerk, vgl. Heinsberg \& Rehbold 2011, 35f.). Abbildung 1 auf der folgenden Seite fasst das idealtypische Laufbahnkonzept grafisch zusammen.

\subsection{Zentrale Funktionen}

\section{Erhöhung der Transparenz im Berufsbildungssystem}

Berufslaufbahnkonzepte können also einen systematischen Überblick zu sämtlichen Qualifizierungsoptionen in einem Beruf bzw. in mehreren verwandten Berufen leisten. Dies kann bildungspolitischen Entscheidungsträgern als Orientierungspunkt für die Neugestaltung von Ordnungsmitteln dienen, zum Beispiel für die Entwicklung oder Zusammenlegung von Ausbildungsordnungen, für die Gestaltung neuer Qualifizierungsbausteine oder für eine bessere Verzahnung verschiedener Qualifizierungsabschnitte an den Schwellen des Berufsbildungssystems.

Darüber hinaus verdeutlicht das idealtypische Laufbahnmodell, dass Berufslaufbahnkonzepte potenziellen Bildungsteilnehmenden in Berufsvorbereitung, Ausbildung, Weiterbildung und Studium einen umfassenden Überblick $\mathrm{zu}$ den spezifischen Qualifizierungsmöglichkeiten und -wegen in einer beruf- 
lichen Domäne vermitteln können, da sie im Idealfall sämtliche formale und non-formale Qualifikationsabschnitte eines Berufs und seine Verbindungslinien zu Nachbarberufen abbilden.

\section{Abb. 1: Idealtypisches Laufbahnkonzept mit Pfadmodell zu einer beispielhaft} typischen , akademischen Laufbahn“
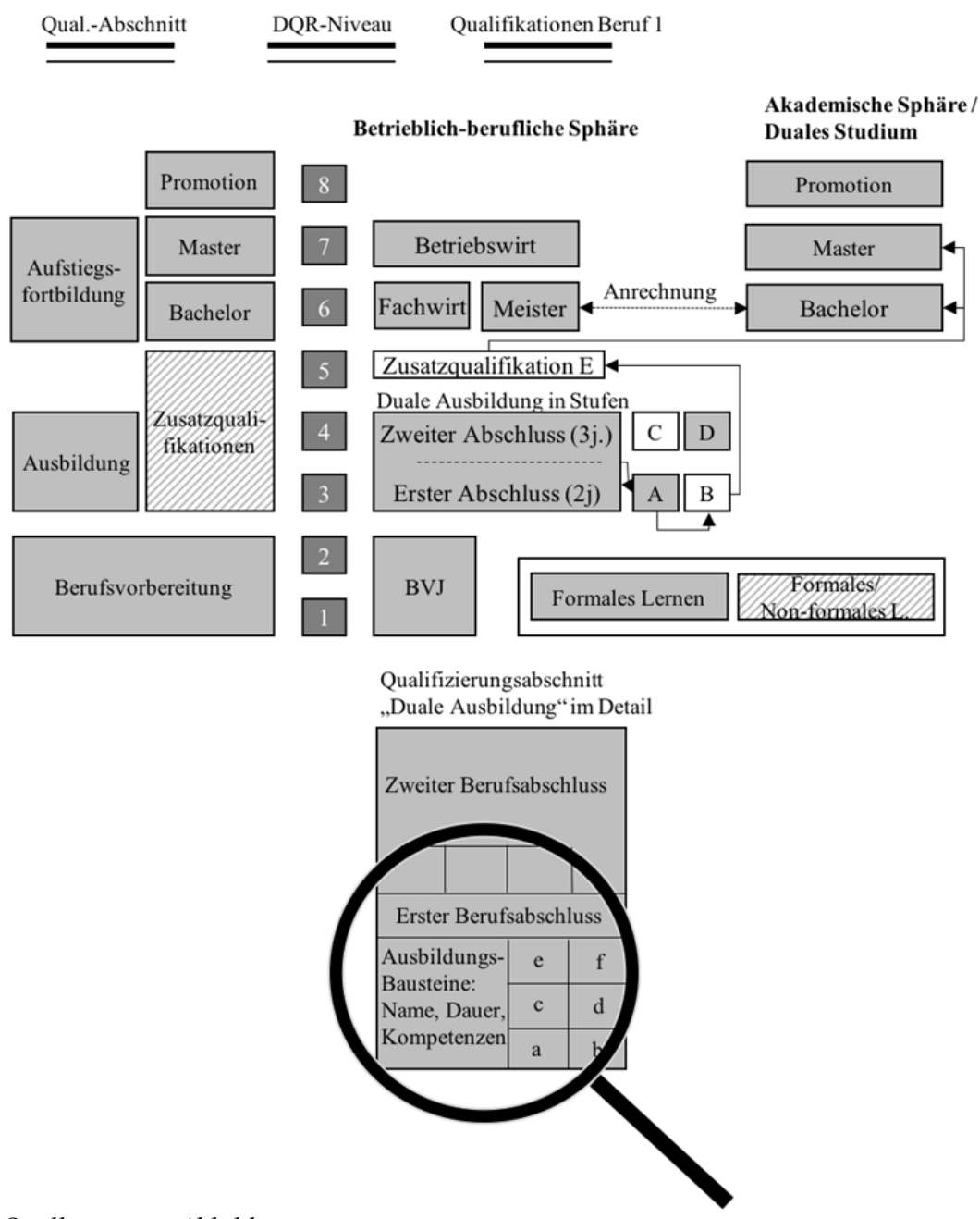

Quelle: eigene Abbildung 
Hierzu sollten Laufbahnkonzepte Informationen über die Dauer einzelner Qualifizierungsabschnitte geben. Zusätzliche Transparenz kann entstehen, wenn die einzelnen Qualifizierungsabschnitte und -bausteine nicht nur nebenund übereinander abgebildet, sondern darüber hinaus miteinander in Beziehung gesetzt werden. In anderen Worten geht es darum, „typische“ Karrierewege unter Berücksichtigung formaler und non-formaler Qualifikationen aufzuzeigen. Damit können individuelle Laufbahnen frühzeitig geplant bzw. Laufbahnplanungen durch einen Abgleich der eigenen Ziele und Wünsche mit den Angeboten des Bildungssystems sowie den betrieblichen Bedarfen angepasst werden.

Die Erzeugung von Transparenz im Berufsbildungssystem durch Berufslaufbahnkonzepte kann zu einem Imagegewinn der dualen Ausbildung führen, die den zentralen Kern aller Berufslaufbahnkonzepte bildet. Insbesondere das Handwerk setzt auf diesen Imagegewinn, da es sich in der öffentlichen Wahrnehmung unterschätzt sieht (vgl. ZDH 2004, 19).

Ein grundsätzlicher Attraktivitätsgewinn der dualen Ausbildung durch Berufslaufbahnkonzepte besteht darin, dass es für breitere Gruppen mit jeweils ganz differenten Leistungsstärken geöffnet werden kann - also etwa von unten durch eine bessere Verzahnung mit der Berufsvorbereitung für schwächere Jugendliche und von oben durch Studienabbrecher/-innen bzw. bildungsaffine Absolventen/-innen des Gymnasiums. Die Abbildung der Laufbahnkonzepte kann hier freilich nur einen kommunikativen Beitrag leisten. Das tatsächliche Potenzial der Öffnung der dualen Ausbildung steckt in der Anregung ordnungspolitischer Prozesse. Zur Erhöhung der Transparenz für Bildungsnachfragende müssen Laufbahnkonzepte zudem in entsprechende Informationsmaterialien überführt und schließlich verbreitet werden.

\section{Weiterentwicklung des Berufsbildungssystems - Ordnungspolitik}

Durch die umfassende Dokumentation von Qualifizierungswegen sind Berufslaufbahnkonzepte geeignet, Sollbruchstellen im Sinne von Qualifizierungslücken oder -redundanzen in Berufsvorbereitung, Berufsausbildung, Studium und Weiterbildung aufzuzeigen. Darüber hinaus können sie verdeutlichen, wo fakultative Qualifizierungen, etwa der Erweiterungsfortbildung auf Gesellenebene, quasi-obligatorischen Charakter erhalten, wenn sie für eine bestimmte berufliche Laufbahn notwendig sind (ein Beispiel hierfür wäre der Betriebsassistent im Kfz-Handwerk, der parallel zur Ausbildung absolviert werden kann).

Berufslaufbahnkonzepte knüpfen direkt an der Durchlässigkeitsdiskussion an und formulieren so ordnungspolitische Ansprüche. Eine Flexibilisierung von Zugangs- und Ausbildungswegen sowie eine stärkere Verzahnung von Aus- und Weiterbildungsangeboten wird seit Jahrzehnten als probate Reaktion auf die oben genannten Entwicklungen (veränderte Qualifizierungsanforderungen, verändertes Bildungsverhalten, Europäisierung, demo- 
grafischer Wandel etc.) betrachtet (vgl. z.B. Euler im Ersch.; Münk 2008, 285f.; Wissenschaftsrat 2014). Insofern stellen Berufslaufbahnkonzepte konkrete Modelle dar, auf deren Basis die alte Debatte um die Gleichwertigkeit beruflicher und allgemeiner Bildung weitergeführt wird (vgl. ZDH 2007, 4; Münk 2012).

Eine Identifizierung gemeinsamer Kompetenzbündel lässt sich für die ordnungspolitische Arbeit nutzen, etwa bei der Formulierung gemeinsamer Lernfelder für den schulischen Teil der dualen Ausbildung. Dies wiederum begünstigt, entsprechende betriebliche Karrieremuster vorausgesetzt, die horizontale Mobilität zwischen artverwandten Berufen. Neben der Durchlässigkeit zwischen Ausbildungsberufen bezieht sich die horizontale Durchlässigkeit insbesondere auf Zusatzqualifikationen während der Ausbildung und sämtliche Fortbildungen, die keinen Übergang auf eine höhere DQRNiveaustufe vorsehen. Auch im Tertiärbereich ist horizontale Durchlässigkeit, etwa zwischen dualen und rein akademischen Studienangeboten, denkbar.

Hinsichtlich der vertikalen Durchlässigkeit dienen Laufbahnkonzepte als Folie, auf der Zugangskriterien für den vertikalen Aufstieg im Berufsbildungssystem diskutiert werden können. Laufbahnkonzepte können insbesondere dabei helfen, Qualifizierungsbausteine auf verschiedenen DQR-Stufen didaktisch-curricular miteinander zu verzahnen, indem inhaltliche Überschneidungspotenziale, z.B. zwischen der Meisterebene und dem akademischen Bereich, identifiziert und Bedingungen für die Anrechnung ausgehandelt werden, um die Übergänge zwischen den einzelnen Stufen zu erleichtern und Qualifizierungsabschnitte zu verkürzen. Ein Beispiel für diese curriculare Verzahnung ist der Übergang von der Meister- zur Hochschulebene im Orthopädietechnik-Handwerk, wobei Teile der Meisterprüfung auf den Bachelorstudiengang Orthopädie-Ingenieur angerechnet werden und im Rahmen des genannten Bachelorstudiengangs auch die Meisterqualifikation erreicht werden kann. Dieses Beispiel macht zugleich deutlich, dass die Verzahnung prinzipiell sowohl aufwärts wie auch abwärtsgerichtet ist. Durch die Explosion der Studienanfänger/-innen und den demografischen Wandel stellen, wiederum besonders für das Handwerk, insbesondere auch Studienabbrecher/innen eine attraktive Zielgruppe für duale Ausbildungsangebote dar (vgl. ZDH 2013). Grundsätzlich bildet die Erleichterung des Hochschulübergangs ein zentrales Motiv der Berufslaufbahnkonzepte. Die Attraktivität des Dualen Systems, so die Prämisse, steigt insbesondere für bildungsaffine Personen, wenn umfassende Aufstiegsmöglichkeiten bis hin zu Arbeitsmarktpositionen bestehen, die einen Hochschulabschluss erfordern und entsprechend berufsbezogene tertiäre Bildungsgänge verfügbar sind. 


\section{Chancen und Grenzen beruflicher Laufbahnkonzepte}

Berufslaufbahnkonzepte machen deutlich, wie sich Erstausbildung, Aufstiegsfortbildung und Hochschulbildung bezogen auf den DQR berufsfeldspezifisch systematisieren und verzahnen lassen. Lernenden und bildungspolitischen Entscheidungsträgern kann so aufgezeigt werden, inwiefern Wege in die tertiäre Bildung sowie grundsätzlich auch berufliche Aus- und Weiterbildungsprozesse flexibilisierbar sind. Je nachdem, ob sie nur als Mittel zur Transparenzschaffung oder als Rahmen für ordnungspolitische Reformprozesse genutzt werden, können Laufbahnkonzepte die Attraktivität beruflicher Bildung vor dem Hintergrund der Akademisierung durchaus steigern, indem sie über Karriereoptionen informieren oder letztere neu begründen. Durch ihren bildungsgangübergreifenden Systematisierungscharakter eignen sie sich zudem dazu, auf Sollbruchstellen im Sinne von Qualifizierungslücken oder redundanzen hinzuweisen.

Allerdings werden bei eingehender Reflexion der Situation beruflicher Bildung zwischen Wissensgesellschaft und demografischem Wandel zentrale Anforderungen an die Gestaltung beruflicher Aufstiegsoptionen deutlich, die berufliche Laufbahnkonzepte, so wie sie aktuell existieren, nicht erfüllen können: Sie müssen zwischen betrieblichen und subjektiven Nutzenkalkülen sowie organisationalen Gegebenheiten betrieblicher Arbeit einerseits und Organisationsformen beruflicher Aus- und Weiterbildungsprozesse andererseits vermitteln. Eigenlogiken der beruflichen Erstaus- und Aufstiegsfortbildung müssen überwunden und berufliche Bildungsoptionen mit betrieblichen Karrierewegen verzahnt werden. Ob eine solche Vermittlung möglich ist, differiert je nach Branche, Beruf und sogar zwischen Unternehmen. Konkretes branchenspezifisches Rekrutierungsverhalten sowie die Rolle von Weiterbildungspatenten beim beruflichen Aufstieg werden in diesem Kontext zu zentralen Forschungsdesiderata. $\mathrm{Zu}$ fragen wäre etwa, inwiefern dem Dualen System ein Attraktivitätsschub verschafft werden kann, indem man die mittleren DQR-Niveaustufen (4-6) zu Durchgangsstationen erklärt und inwiefern die Studienorientierung in den Konzepten den heterogenen betrieblichen Qualifizierungs- und Einsatzmustern entgegenkommt. Ferner setzen Pfadmodelle zu beruflichen Laufbahnen umfangreiches empirisches Wissen über berufliche Karrieremuster voraus.

An dieser Stelle lässt sich die zentrale Schwäche von Berufslaufbahnkonzepten benennen: Als mehr oder weniger komplexe Modelle zur Darstellung des status quo bzw. von Zukunftsszenarien ohne genuin neue Lösungsansätze für zentrale berufsbildungspolitischen Fragestellungen verdeutlichen sie letztlich nur die gleichen Probleme, die auch in den jeweiligen Teildiskussionen um die Europäisierung, Durchlässigkeit, Transparenz, Anerkennung non-formalen Lernens usw. bislang ungelöst sind. 
Für die Erzeugung von Durchlässigkeit an der Schnittstelle von der Fortbildungsebene zum Studium etwa genügt die Betrachtung der in den Laufbahnkonzepten abgebildeten Struktur bei weitem nicht. Vielmehr müssen sämtliche an den jeweiligen Qualifizierungsabschnitten beteiligten Institutionen (Sozialpartner, Hochschulen, Politik, Forschung) gemeinsam Lösungen zur Verbesserung der Durchlässigkeit erarbeiten. Die Berufslaufbahnkonzepte können hier lediglich Ausgangspunkt für die Identifikation von Bedarfen und Endpunkt, nämlich über die Abbildung von neuen Verzahnungen sein. Die zahlreichen mit der Aufstiegsorientierung verbundenen Probleme machen die Grenzen von Berufslaufbahnkonzepten deutlich und verweisen auf die erheblichen Anstrengungen, die zur Verbesserung der bildungspolitischen Rahmenbedingungen zu leisten sind. So bergen schon die Fragen, inwiefern inhaltliche Dubletten in Ausbildung und Studium (Beispiel: Betriebliches Rechnungswesen) tatsächlich reduziert werden können und ob ein Verzicht auf die Wissensvermittlung eher die berufspraktische oder die akademische Sphäre treffen soll, erhebliches Konfliktpotenzial. Darüber hinaus sind nicht nur tertiäre Bildungsgänge mit der Aufstiegsfortbildungsebene zu verzahnen, sondern insbesondere auch Aspekte der Finanzierbarkeit und der Wissenschaftspropädeutik zu klären. Schließlich nehmen die Laufbahnkonzepte hier Erwachsene in den Blick, die bereits lange im Berufsleben stehen, durch ihre besondere betrieblich-berufliche Sozialisation keinen leichten Anschluss an die akademischen Denk- und Arbeitsmuster erhalten und zumeist in der Familiengründungsphase sind oder diese bereits abgeschlossen haben. Duale Studiengänge und stark modularisierte Studienprogramme, wie sie sich in der wissenschaftlichen Weiterbildung immer stärker durchsetzen, scheinen für diese Gruppe deutlich besser geeignet. Rahmenkonzeptionen für berufliche Laufbahnkonzepte, wie das von Rauner (2012) oder Euler (im Ersch.), die klassischen generischen bzw. rein akademischen Studiengängen integrierte Studiengänge gegenüberstellen, greifen die Idee einer konsequent dualen beruflichen Bildung vom Dualen System bis zum Masterabschluss auf.

Auch in Bezug auf Teilqualifikationen stoßen Laufbahnkonzepte, die für Teilzertifizierung definitiv offen sind, schnell an ihre Grenzen. Euler und Severing (2007) haben verdeutlicht, dass sich Modularisierung und Beruflichkeit nicht widersprechen. Dennoch ist in der Debatte derzeit wenig Bewegung. Bezogen auf die am Anfang von Kapitel 3 diskutierten Funktionen wird also deutlich, dass am ehesten ein Transparenzgewinn für Fortbildungswillige von beruflichen Laufbahnkonzepten erwartbar ist, sowie ordnungspolitische Impulse in Richtung Durchlässigkeit in die tertäre Bildung und eine verbesserte Verzahnung beruflicher Erstausbildung mit der Aufstiegsfortbildung.

Der Erfolg von Berufslaufbahnkonzepten hängt letztlich davon ab, inwieweit es möglich ist, die Eigenlogik bestehender Strukturen wie Systeme beruflicher Erstausbildung und Weiterbildung sowie betriebliche Karriere- 
modelle aufzubrechen und so zu gestalten, dass Individuen überhaupt erst in die Lage versetzt werden, den Anforderungen von Wissensgesellschaft und demografischem Wandel gerecht zu werden. Derzeit scheint ihnen ein mit dem DQR vergleichbares Schicksal zuteil zu werden: Einerseits sind sie brauchbare Instrumente - sozusagen bildungspolitische Katalysatoren -, die dabei helfen, zentrale Diskussionslinien zu bündeln und am Leben zu erhalten. Andererseits gelingt es ihnen kaum, die Diskussionen auch tatsächlich weiterzutreiben.

\section{Literatur}

Autorengruppe Bildungsberichterstattung (2014). Bildung in Deutschland 2014. Bielefeld: wbv.

Baethge, M., Kerst, C., Leszczensky, M. \& Wieck, M. (2014). Zur neuen Konstellation zwischen Hochschulbildung und Berufsausbildung. Forum Hochschule 3. Online: http://www.dzhw.eu/pdf/pub_fh/fh-201403.pdf (02.11.2015).

Baethge, M. \& Wieck, M. (2015). Neue Konstellation zwischen Berufsausbildung und Hochschulstudium. Wendepunkt in der deutschen Bildungsgeschichte. Mitteilungen aus dem SOFI, 22, 2-5.

Becker, M. (2011). Berufslaufbahnkonzepte im Handwerk und Karriereoptionen. bwp@ Sonderheft 5 - Hochschultage Berufliche Bildung 2011, WS 26, hrsg. v. Becker, M./Krebs, R./Spöttl, G., 1-15.Online:http://www.bwpat.de/ht2011/ws26/ becker_ws26-ht2011.pdf (03.12.2015).

BLK-DQR ${ }^{-}$(2013). Handbuch zum Deutschen Qualifikationsrahmen. Online: http://www.kmk.org/fileadmin/pdf/PresseUndAktuelles/2013/131202_DQR-Handbuch_M3_pdf (03.12.2015).

Born, V. (2012). Das Berufslaufbahnkonzept im Handwerk. $b w p, 4,45-48$.

Dobischat, R. \& Schurgatz, R. (2015). Informelles Lernen: Chancen und Risiken im Kontext von Beschäftigung und Bildung. In G. Niedermair (Hrsg.), Informelles Lernen. Annäherungen - Problemlagen - Forschungsbefunde (S. 27-42). Linz: Trauner.

Esser, F. (2003). Berufsbaukästen im Handwerk. Kölner Zeitschrift für Wirtschaft und Pädagogik, 18(34), 205-228.

Euler, D. (im Ersch.). Gleichartig aber nicht gleichwertig? Überlegungen zu einer Neubestimmung des Verhältnisses zwischen Berufs- und Hochschulbildung. In D. Münk \& M. Walter (Hrsg.), Die Verheißung vom Lebenslangen Lernen: Berufsbiografische Ambivalenzen in der Moderne. Wiesbaden: VS.

Euler, D. \& Severing, E. (2007). Flexible Ausbildungswege in der Berufsbildung. Bielefeld: wbv.

Gerds, P. \& Spöttl, G. (2010). Entwicklungstendenzen des deutschen Berufsbildungssystems und Folgerungen für die duale Ausbildung im Handwerk. ITB Forschungsbericht 48. Bremen: ITB. 
Heinsbeg, T. \& Rehbold, R. (2011). Transparenz der Aus- und Weiterbildungsstrukturen sowie der Karrierewege im Gesundheitshandwerk unter Anwendung des modifizierten Berufslaufbahnkonzepts im Handwerk. DHI-Arbeitshefte zur berufs- und wirtschaftspädagogischen Forschung. Köln: DHI.

Lipsmeier, A. \& Münk, D. (1997). Berufliche Weiterbildung. Hohengehren: Schneider.

Maier, M. \& Vogel, T. (2013). Blinde Flecke in der Debatte zum Übergangssystem Schule - Beruf. In M. Maier \& T. Vogel (Hrsg.), Übergänge in eine neue Arbeitswelt? Blinde Flecke in der Debatte zum Übergangssystem Schule - Beruf, (S.9-26). Wiesbaden: VS Verlag für Sozialwissenschaften.

Münk, D. (2012). Deadman Talking: Anmerkungen zur Akademisierung der Berufsbildung. berufsbildung, 136, 2-4.

Münk, D. (2008). Standards in der beruflichen Bildung und der EQR: Anmerkungen zur bemerkenswerten Karriere eines europäischen Konzepts. In U. Faßhauer, D. Münk, A. Paul-Kohlhoff (Hrsg.), Berufspädagogische Forschung in sozialer Verantwortung, (S. 273-293). Stuttgart: Franz Steiner.

Pongratz, H.-J. \& Voß, G. (2000). Vom Arbeitnehmer zum Arbeitskraftunternehmer Zur Entgrenzung der Ware Arbeitskraft. In H. Minssen (Hrsg.), Begrenzte Entgrenzung: Wandlungen von Organisation und Arbeit, (S. 125-143). Berlin: edition sigma.

Rauner, F. (2012). Akademisierung beruflicher und Verberuflichung akademischer Bildung - widersprüchliche Trends im Wandel nationaler Bildungssysteme. bwp@Berufs-und Wirtschaftspädagogik-online, 23, 1-19. Online: http://www. bwpat.de/ausgabe23/rauner_bwpat23.pdf (03.12.2015).

Schmidt, C. (2012). Krisensymptom Übergangssystem. Bielefeld: wbv.

Severing, E. \& Teichler, U. (2013). Akademisierung der Berufswelt? Verberuflichung der Hochschulen? In E. Severing \& U. Teichler (Hrsg.), Akademisierung der Berufswelt?, (S. 7-18). Bielefeld: Bertelsmann.

Walter, M. (2014). Lebenslanges Lernen zwischen Weiterbildungslust und Weiterbildungsfrust. Bielefeld: wbv.

Wissenschaftsrat (2014). Empfehlungen zur Gestaltung des Verhältnisses von beruflicher und akademischer Bildung. Drs. 3818-14. Darmstadt: WR.

ZDH (2013). Integration von Studienaussteigern in das duale Berufsbildungssystem. Berlin: ZDH. Online: https://www.zdh.de/fileadmin/user_upload/themen/Bildung/Fachkraeftesicherung/Auswertung_Studienaussteiger_Fachkraeftepotenzial 09-2013.pdf (15.03.2016).

ZDH (2007). Ganzheitlich, Passgenau, Anschlussfähig. Grundzüge eines umfassenden und flexiblen Berufslaufbahnkonzepts im Handwerk. Berlin: ZDH. Online: https:/www. zdh.de/fileadmin/user_upload/themen/Bildung/Rundschreiben_200 8/080305Berufslaufbahnkonzept.pdf $(03.12 .2015)$.

ZDH (2004). Differenzierung und Europäisierung der beruflichen Bildung. Schriftenreihe des ZDH, Heft 61. Berlin: ZDH. 


\section{Gestaltungsfragen zur Akademisierung der beruflichen Bildung am Beispiel der Gesundheitsfachberufe}

Karl-Heinz Gerholz, Ursula Walkenhorst

\section{Akademisierungstendenzen in der beruflichen Bildung}

In den letzten Jahren können am oberen Rand der Berufsausbildung „Erosionstendenzen" (Euler 2014, 323) beobachtet werden. Jugendliche mit Hochschulzugangsberechtigung entscheiden sich eher für ein Bachelor-Studium an einer Hochschule als für eine Berufsausbildung im Dualen System. Ursachen aus Perspektive des Bildungssystems sind u. a. die mit einem Hochschulstudium verbundenen besseren Karriereperspektiven (z.B. Einkommen, berufliche Position). Aus Perspektive des Beschäftigungssystems ist eine Entwicklung $\mathrm{zu}$ einer Servicegesellschaft $\mathrm{zu}$ beobachten und damit einhergehend wird ein stärkerer Bedarf an akademischen Fähigkeiten nachgefragt (vgl. Baethge \& Wolter 2015, 6ff.; Euler 2014, 322ff.). Die skizzierten Veränderungen zwischen Berufsbildung und Hochschubildung zeigen auf, dass es einer Neubestimmung der beiden Bereiche zueinander bedarf (vgl. AGBB 2014, 12; Baethge \& Wolter 2015).

Im folgenden Beitrag wird dieser Frage am Beispiel der Ausbildung in den therapeutischen Gesundheitsfachberufen ${ }^{1}$ nachgegangen. Zwar stellen diese Berufe eine Sonderform der deutschen Berufsausbildung ${ }^{2}$ dar, gleichzeitig sind sie aber Referenzbeispiel der Akademisierung beruflicher Bildung auf Hochschulebene. Letzteres hängt mit der Modellklausel des Bundes aus dem Jahre 2009 (vgl. GEBK 2009) zusammen, die es ermöglicht, neben der berufsfachschulischen Ausbildung auch auf Hochschulebene auszubilden. Intention ist ein stärker wissenschaftlich orientierter Kompetenzentwicklungsprozess bei den Lernenden, um eine Professionalisierung der Berufsbil-

1 Wir fokussieren nachfolgend die therapeutischen Gesundheitsfachberufe (Ergotherapie, Logopädie, Physiotherapie), da diese auch Gegenstand der Modellklausel im Jahre 2009 sind. Durchaus wären auch die Pflegeberufe näher in den Blick zu nehmen, wo bereits seit 2003 mit Einführung des damaligen Gesundheits- und Krankenpflegegesetz eine Akademisierung ermöglicht wurde, aber diese sind in ihrer Differenzierung mit anderen Fragestellungen verbunden.

2 So existiert $u$. a. keine Dualität in den ordnungspolitischen Grundlagen, da die Berufsgesetze auf Bundesebene erlassen werden, die Umsetzung jedoch durch länderpolitische Vorgaben erfolgt und es ist keine geregelte Lehrerbildung in den einzelnen Bundesländern zu beobachten. 
der im Gesundheitssektor zu erreichen. Hierzu haben sich in den letzten Jahren unterschiedliche Studiengangskonzepte herausgebildet:

(1) primärqualifizierende Studiengänge, bei denen die Ausbildung inklusive der Praxisanteile auf Hochschulebene verankert ist,

(2) ausbildungsintegrierende Studiengänge, bei denen eine Verzahnung von schulischen und hochschulischen Ausbildungsanteilen stattfindet und

(3) additive Studiengänge, bei denen zwei Curricula jeweils auf Hochschulebene und schulischer Ebene vorhanden sind und gegenseitige Anerkennungsrichtlinien die Ausbildung regeln (vgl. WR 2012, 56ff.).

Die Studiengangskonzepte zeigen auf, dass Strukturen der Berufsausbildung mit Strukturen der Hochschulbildung verzahnt werden. Mit der Modellklausel geht aber keine formale Veränderung des Berufsbildes einher, sondern die berufsgesetzlichen Regelungen bleiben auf Hochschulebene bestehen. Dies ermöglicht die Vergabe einer Doppelqualifikation - nämlich der originären Berufszulassung sowie des akademischen Grades eines Bachelorabschlusses. Daraus resultieren spezifische Gestaltungsfragen, v. a. hinsichtlich der Bestimmung von beruflicher Bildung und Hochschulbildung.

Interesse des vorliegenden Beitrages ist es, den ,state of the art' der Akademisierung der Gesundheitsfachberufe zu analysieren, um aktuelle Herausforderungen und deren Gestaltungsansätze herauszuarbeiten. Leitend bei der Analyse sind dabei die Handlungsebenen der beruflichen Bildung. Auf der Makroebene geht es um das strukturelle Verhältnis von beruflicher Bildung und Hochschulbildung auf Ebene des Bildungs- und Beschäftigungssystems (Abschnitt 2). Auf der Mesoebene geht es um den Aspekt des curricularen Aufbaus der Studiengänge und der Studiengangentwicklung (Abschnitt 3). Auf der Mikroebene steht die Frage der (fach)didaktischen Gestaltung der Kompetenzentwicklung der Studierenden im Mittelpunkt und wie das Spannungsfeld des berufspraktischen wie forschungsorientierten Anspruches auszutarieren ist (Abschnitt 4). Die Ausführungen münden in eine $\mathrm{Zu}$ sammenführung der Analyseergebnisse und welche Gestaltungsansätze sich daraus v.a. in Hinblick auf den internationalen Diskurs ergeben (Abschnitt 5).

\section{Makroebene: Zielstellung der Akademisierung}

Die Akademisierung in den Gesundheitsfachberufen begründet sich sowohl aus Anforderungen des Beschäftigungssystems hinsichtlich der Sicherung zukünftiger Versorgungsstrukturen in der Gesellschaft als auch aus Anforde- 
rungen des Bildungssystems hinsichtlich des Aufbaus von Wissenschaft und Forschung in neuen Disziplinen.

In Perspektive des Beschäftigungssystems können zunächst demografische und epidemiologische Veränderungen angeführt werden. Die Versorgungssituation der Zukunft ist durch eine Zunahme älterer Menschen in der Bevölkerung, chronischen Erkrankungen und Multimorbidität geprägt, die einen höheren Pflege- und Betreuungsaufwand zur Folge hat (vgl. SVR 2009). Dabei müssen zukünftige Versorgungsstrukturen nicht nur integrierte sektorale Versorgungskonzepte aufzeigen, sondern zielgruppenspezifische und generationsspezifische Versorgungskonzepte bedenken (vgl. Walkenhorst 2011, 2f.). Weiterhin steigen die Qualifikationserfordernisse in der professionellen Arbeit hin zu einer stärkeren Evidenzbasierung und die interprofessionelle Zusammenarbeit gewinnt zunehmend an Bedeutung, was u. a. Bourgeault, Kuhlmann, Neitermann und Wrede (2008) in einer europäischen Ländervergleichsstudie herausgearbeitet haben (vgl. u. a. auch WR 2012). Dem evidenzbasierten und interprofessionellen Handeln kommt somit eine wichtige Bedeutung zu. Demgegenüber ist aber festzuhalten, dass in der Praxis der Gesundheitseinrichtungen die Gesundheitsfachberufe in der $\mathrm{Zu}-$ sammenarbeit mit der medizinischen Disziplin meist belastet sind. Die Strukturen in Gesundheitseinrichtungen sind nach wie vor stark hierarchisiert und durch eindeutige rechtliche Vorgaben gekennzeichnet, weshalb die Gesundheitsfachberufe sich i. d. R. in der Rolle des ,mitarbeitenden' und ,hilfsarbeitenden' Personals befinden (vgl. Walkenhorst 2015, 7). Mit anderen Worten kann das Ziel der Akademisierung, nämlich autonom und evidenzbasiert gesundheitsbezogene Leistungen vorzunehmen, in der Realität der Arbeitsprozesse aufgrund der traditionellen Hierarchisierung noch keine steuernde Wirkung entfalten.

Aus Perspektive des Bildungssystems soll über die Akademisierung der Gesundheitsfachberufe einerseits der Aufbau von Wissenschaft und Forschung im Gesundheitsbereich gestaltet werden, um aktuelle und zukünftige Gesundheitsfragestellungen wissenschaftlich fundiert zu beantworten. Damit einhergehen Fragen des Aufbaues der jeweiligen Disziplin in den Gesundheitsfachberufen sowie der Bestimmung des Verhältnisses zur Medizin (vgl. Walkenhorst 2015, 5ff.). Andererseits soll eine internationale Anbindung der Berufsbildung im Gesundheitsbereich erreicht werden. Die Etablierung von Bachelor- und Master-Studiengängen im Gesundheitsbereich ist im europäischen und außereuropäischen Raum bereits vor Jahrzehnten vorgenommen worden, wenngleich die Ausbildungsstrukturen immer im Lichte der jeweiligen länderspezifischen Gesundheitssysteme $\mathrm{zu}$ betrachten sind (vgl. Walkenhorst 2011, 4).

Wird der Blick auf die aktuelle Situation in Deutschland gerichtet, so kann die Etablierung von Bachelor- und Master-Studiengängen im Sinne einer Akademisierung zwar beobachtet werden, gleichzeitig bestehen aber 
die Berufsgesetze in der hochschulischen Qualifizierung fort. Die ordnungspolitischen Grundlagen sind auf schulischer und hochschulischer Ebene strukturell gleich, aber das Abschlussniveau ist verschieden. Dies führt zu Fragen der Einordnung der entstehenden Berufsprofile im Bildungs- und Beschäftigungssystem. So wird z.B. der Bachelor-Abschluss im aktuellen Entwurf des Deutschen Qualifikationsrahmens einheitlich auf Stufe 6 eingeordnet. Ein Physiotherapeut mit Bachelor-Abschluss wäre somit auf Stufe 6 einzuordnen, während ein Physiotherapeut mit klassischer berufsfachschulischer Bildung darunter liegt (aktuell Stufe 4), obwohl beide Ausbildungsstrukturen denselben berufsgesetzlichen Regelungen unterliegen. Auch stellt sich die Frage, wie die unterschiedlichen Kompetenzprofile in den Arbeitsprozessen der Gesundheitseinrichtungen zukünftig eingeordnet werden, v. a. in Hinblick auf die vorliegende Hierarchisierung in den Arbeitsprozessen.

In Zusammenführung der Bestandsaufnahme auf der Makroebene können drei Herausforderungen jeweils zwischen den Perspektiven Beschäftigungssystem, Bildungspolitik und Bildungssystem (Ebene Hochschule) festgehalten werden: ${ }^{3}$

1. Verbindung Bildungspolitik - Bildungssystem (Ebene Hochschule): Durch die Beibehaltung der berufsgesetzlichen Vorgaben, welche allgemein im Gesetzgebungsbereich des Bundes liegen, stellt die Akademisierung vielmehr eine formale Anhebung der Ausbildungsvoraussetzungen dar, ohne dass sich dabei aber ordnungspolitisch betrachtet die Berufsprofile ändern. Es ist aber anzunehmen, dass die entstehenden Kompetenzprofile durch akademische Merkmale gekennzeichnet sind.

2. Verbindung Bildungssystem (Ebene Hochschule) - Beschäftigungssystem: Unklar ist, welche beruflichen Einsatzfelder mit einem Hochschulabschluss verbunden sind. Durch die Hierarchisierungen in den Gesundheitseinrichtungen können sich die akademischen Fähigkeiten der Absolventen in den Gesundheitsfachberufen nur bedingt entfalten. Das Beschäftigungssystem scheint auf die neuen Ausbildungsstrukturen noch nicht vorbereitet und es besteht die Gefahr, dass Bachelor-Absolventen klassische Berufsfelder der bisherigen Absolventen mit berufsfachschulischer Ausbildung einnehmen, was letztlich eine Dequalifizierung des Bachelor-Abschlusses darstellen würde und damit zu einer Form der DeProfessionalisierung führen kann (vgl. Walkenhorst 2008).

3. Verbindung Beschäftigungssystem - Bildungspolitik: Durch die Modellklausel wird zwar eine Akademisierung ermöglicht, allerdings bleiben durch die Berufsgesetze die Berufsprofile in ihren Grundlagen bestehen.

3 Vgl. dazu auch Wolter (2015), welcher sechs allgemeine Problemfelder der gegenwärtigen Akademisierung herausarbeitet. 
Es besteht die Gefahr einer vertikalen Substitution, indem z.B. Physiotherapeuten mit Bachelor-Abschluss Physiotherapeuten mit einer klassischen berufsfachschulischen Ausbildung verdrängen könnten. Es findet somit keine wirkliche bildungspolitische Steuerung statt, indem z.B. die Berufsgesetze auf Basis der akademischen Anforderungen im Gesundheitsbereich angepasst werden und unterschiedliche Berufsprofile (z.B. in Orientierung zu den Assisstentenberufen in Skandinavien oder den USA) ermöglicht werden.

Abb. 1: Herausforderungen auf der Makroebene

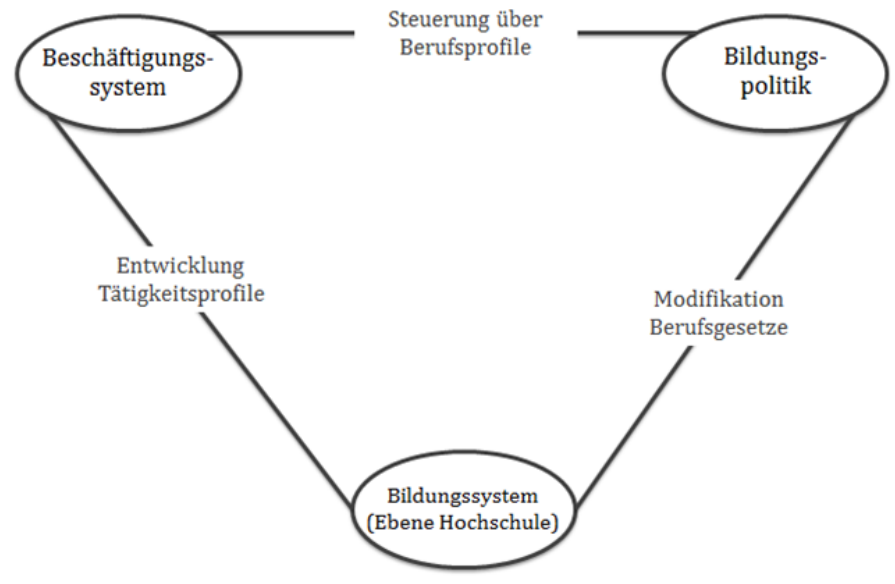

Quelle: eigene Abbildung

Abbildung 1 visualisiert die Zusammenhänge der aufgezeigten Herausforderungen. Hierbei wird deutlich, dass eine strukturiertere Abstimmung zwischen den Akteuren aus Bildungspolitik, Bildungssystem (Ebene Hochschule) und Beschäftigungssystem erforderlich erscheint, um einen kohärenten Akademisierungsprozess der Gesundheitsfachberufe zu erreichen. Als ein Prototyp zur Organisation solcher Abstimmungsprozesse können die korporatistischen Strukturen der dualen Ausbildung betrachtet werden, indem Bund und Länder sowie Arbeitgeber und Arbeitnehmer in gemeinsamer Abstimmung eine Steuerung der dualen Berufsausbildung vornehmen. Hierzu wurden entsprechende organisatorische Elemente (z.B. Hauptausschuss des Bundesinstituts für berufliche Bildung) geschaffen. Für die Akademisierung im Bereich der Gesundheitsfachberufe kann die Etablierung korporatistischer Strukturen ein Element darstellen, um eine stärkere Verzahnung der Qualifizierungsstrukturen zu ermöglichen. Hiermit wären gleichwohl Gestaltungs- 
fragen zu klären, wie u. a. Hochschulen als autonome Bidlungsorganisationen sich in ein korporatistisches Gefüge einbetten lassen und inwiefern dieses Gefüge Veränderungen anstoßen kann (z.B. hinsichtlich der vorherrschenden Hierarchisierung in den Gesundheitseinrichtungen). Anders gesagt, geht es darum, die korporatistischen Strukturen der dualen Berufsausbildung als eine Schablone zu betrachten, um die Aushandlungsprozesse zwischen Gesundheitseinrichtungen (Beschäftigungssystem), Regularien (Bildungspolitik) sowie Bildungsorganisationen wie Hochschulen und Berufsfachschulen (Bildungssystem) zu organisieren. Diese Notwendigkeit ist im internationalen Raum bereits erkannt worden und hat zur entsprechenden Entwicklung von Diskussionen und Strategien beigetragen (vgl. Sottas, Höppner, Kickbusch, Pelikan \& Probst 2013).

\section{Mesoebene: Curriculare und organisatorische Umsetzung der Akademisierung}

Auf der Mesoebene rückt die curriculare und organisatorische Gestaltung der (Aus)bildungsprozesse in den Mittelpunkt der Betrachtung. Hinsichtlich der curricularen Ebene besteht in der Akademisierung der Gesundheitsfachberufe die Herausforderung, die unterschiedlichen Logiken der beruflichen Bildung bzw. Berufsgesetze und der Hochschulbildung zueinander in Beziehung zu setzen und aufeinander abzustimmen.

In der dualen Berufsausbildung kann das Leitziel in der Förderung einer beruflichen Handlungskompetenz gesehen werden (vgl. KMK 2000). Die curricularen Grundlagen in Form der Rahmenlehrpläne sind lernfeldstrukturiert. Es handelt sich dabei in der Terminologie der KMK um kompetenzbasierte Zielformulierungen, die die beruflichen Handlungsfelder widerspiegeln (vgl. dazu Sloane 2007). Lernfelder sind somit Schneidungen zwischen beruflichen Tätigkeiten und Inhalten, womit eine stärker handlungssysstematische Strukturierung vorliegt. Auf Ebene der Berufsgesetze der Gesundheitsfachberufe ist ein allgemeines Leitziel nicht direkt zu erkennen, vielmehr wird in den Berufsgesetzen die Verbindung von theoretischen und praktischen Unterricht sowie praktischer Ausbildung betont, die curricular in einer fachsystematischen Struktur abgebildet werden (z.B. Anatomie, Physiologie, allgemeine Krankheitslehre) (vgl. PhysTh-APrV, ErgThAPrV, LogAPrO). Nimmt man demgegenüber die Landeslehrpläne4 in den Gesundheitsfachberufen als Grundlage, orientieren sich diese i. d. R. an der Förde-

4 Es wird an dieser Stelle allgemein von Lehrplänen gesprochen, gleichwohl die sprachliche Konnotation in den Bundesländern unterschiedlich ist (z.B. in NRW wird es unter ,Empfehlender Ausbildungsrichtlinie' gefasst). 
rung einer beruflichen Handlungskompetenz und curricular wird eine handlungssystematischen Strukturierung in Orientierung zu den Lernfelder vorgenommen (vgl. u. a. LPPhyBY 2013, LPPhyNRW 2013, LPErgB 2005). Es sind somit Unterschiede in der curricularen Strukturierung zwischen den Berufsgesetzen und den Lehrplanhandreichungen auf Länderebene festzuhalten.

Auf Ebene der Hochschulbildung kann das Leitziel in der Förderung einer wissenschaftlich basierten Handlungskompetenz beschrieben werden. Es geht um die Befähigung der Studierenden, in zukünftigen Handlungssituationen Probleme zu erkennen und für die Problemlösung wissenschaftliche Verfahren anzuwenden und diese zu reflektieren (vgl. Gerholz \& Sloane 2011). Der Wissenschaftsrat formuliert dies im Bild des ,reflective practitioners', indem therapeutisches Handeln auf Basis aktueller wissenschaftlicher Erkenntnisse vorgenommen und reflektiert sowie entsprechend angepasst werden soll (vgl. WR 2012, 78; vertiefend Schön 1984). Auf curricularer Ebene stellen in der Hochschulbildung die strukturierenden Einheiten die Module dar. Die KMK beschreibt Module als Zusammenfassung von Stoffgebieten (vgl. KMK 2004). Es wird somit stärker von einer fachsystematischen Strukturierung ausgegangen. Für die Gesundheitsfachberufe zeigt sich dabei die Herausforderung, dass es keine Tradition eines ,Faches' gibt, sondern vielmehr der Aufbau einer wissenschaftlichen Disziplin ,Gesundheitswissenschaften' bzw. ggf. ,Therapiewissenschaften ' für die Gesundheitsfachberufe in der Entwicklung ist. Damit gehen die Klärung der inhaltlichen Gegenstände und des Selbstverstänndisses sowie die Abgrenzung zu anderen Disziplinen wie der Medizin einher (vgl. Walkenhorst 2015, 6ff.). Die Bestimmung der beruflichen Handlungsfelder und damit einhergehend der zu fördernden Kompetenzprofile über ein Curriculum sind somit im Zusammenhang der Herausbildung einer Disziplin- bzw. Fachstruktur zu sehen. Vor diesem Hintergrund scheint es nicht überraschend, dass in der aktuellen curricularen Umsetzung der Modellstudiengänge in den Gesundheitsfachberufen eine hohe Heterogenität vorliegt. So zeigen Darmann-Finck et al. (2014, 103ff.) in ihrem Gutachten zu den Modellstudiengängen in NordrheinWestfalen auf, dass Umfänge von Modulen und Verteilung von Workloads auf inhaltliche Schwerpunkte zwischen den Studiengängen variieren. Auch zeigt sich selten ein einheitliches curriculares Gestaltungsprinzip, da fach- als auch handlungssystematische Strukturierungen auf Studiengangebene vorgenommen werden. Die Heterogenität führt zu einer geringen Vergleichbarkeit der Studiengänge und es ist zu vermuten, dass dadurch unterschiedliche Kompetenzprofile in einem gleichen Berufsbild entstehen.

Eine weitere Herausforderung ergibt sich auf der organisatorischen Ebene der Studiengänge. Die Berufsgesetze schreiben Praxisanteile an Gesundheitseinrichtungen vor. Es sind somit zwei Lernorte - Hochschule und Ge- 
sundheitseinrichtung - in einem Studiengang zu berücksichtigen. ${ }^{5}$ Hierbei gilt es Organisationsformen $\mathrm{zu}$ finden, die die curriculare und inhaltliche Zusammenarbeit zwischen Hochschule und Praxiseinrichtungen strukturieren. Auch hier zeigt sich, dass aktuell die Zusammenarbeit mit den Gesundheitseinrichtungen und die Integration von Praxisphasen in das Studium unterschiedlich umgesetzt werden: Einerseits in der Sequenzierung von Studiums- und Praxisphasen in einem Studiengang und andererseits in der Abstimmung von Präsenz- und Selbstlernzeiten (vgl. Darmann-Finck et al. 2014, 103ff.). Letzteres wird auch durch die Problematik induziert, dass der Workload in Modulen in Präsenz- und Selbstlernzeiten aufgeteilt ist, während die Berufsgesetze nur von ,Präsenzstunden' ausgehen.

Zusammenfassend ergeben sich auf der Mesoebene zwei Herausforderungen: Zum einen geht es um die Entwicklung und Umsetzung eines kohärenten curricularen Designs - welches die Praxisphasen in den Gesundheitseinrichtungen mit einschließt - auf Studiengangebene. Zum Anderem genau genommen parallel dazu - sind auf organisatorischer Ebene intraorganisationale Formen der Zusammenarbeit und Abstimmung zwischen den Lehrenden bzw. den Bezugsdisziplinen für die Gesundheitswissenschaften zu etablieren. Darüber hinaus gilt es interorganisational in Abstimmung zum curricularen Design Formen der Zusammenarbeit zwischen Hochschule und Gesundheitseinrichtungen zu finden.

Die Herausforderungen verweisen auf die Gestaltung der Studiengangentwicklung, welche einen sozialen Problemlöseprozess zwischen den beteiligten Akteuren (wie Lehrende, Praxiseinrichtungen, Stakeholder) und den gegebenen Kontextstrukturen darstellt (vgl. Cornbleth 1990, 5). Dabei ist zu beachten, dass die Zusammenarbeit an Hochschulen durch die Elemente Autonomie und Unabhängigkeit geprägt ist. Für den Prozess der Studiengangentwicklung bedeutet dies, dass die Freiheiten der beteiligten Akteure möglichst erhalten bleiben, aber gleichzeitig ein Rahmen existiert, der es erlaubt, eine systematische und kohärente Studiengangentwicklung durchzuführen. Hierfür kann die Etablierung von Diskursräumen hilfreich sein, die es zulassen, dass die relevanten Bezugsgruppen ein Studienprogramm aushandeln können. Diskursive Studiengangentwicklung zielt somit auf die Schaffung kommunikativer Verfahren, die eine Teilhabe der in der Curriculumentwicklung relevanten Akteure und deren Positionen ermöglicht. Darüber hinaus ist eine Themenstruktur aufzunehmen, die die Austauschprozesse der curricularen Arbeit strukturiert (vgl. Gerholz \& Sloane 2016, 163ff., Gerholz 2013, 19f.). In Abbildung 2 sind die Zusammenhänge visualisiert.

5 Bei ausbildungsintegrierenden Studiengängen (vgl. Abschnitt 1) sind drei Lernorte zu differenzieren; neben Hochschule und Gesundheitseinrichtungen auch die Schulen des Gesundheitswesens. 
Abb. 2: Gestaltung der Mesoebene: Diskursive Studiengangentwicklung

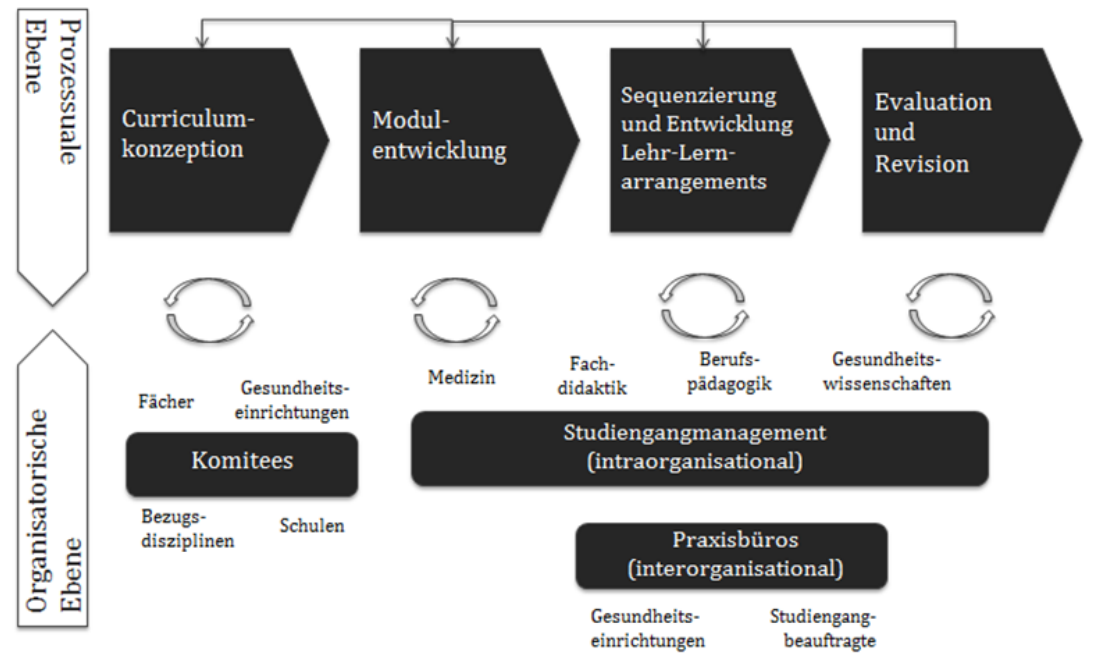

Quelle: in Orientierung zu Gerholz \& Sloane 2016, 164 und Gerholz 2013, 20

Auf prozessualer Ebene sollte eine Themenstruktur für die diskursive Studienprogrammplanung etabliert werden. Die (1) Curriculumkonzeption meint die Aushandlung eines Leitziels für den Studiengang und dieses anschließend in ein curriculares Konzept zu überführen. Letzteres zielt auf das leitende curriculare Gestaltungsprinzip - z.B. handlungssystematische oder fachsystematische Gestaltung - um darauf basierend (2) die Module zu entwickeln. Daran anschließend sind (3) die Module vor dem Hintergrund des intendierten Kompetenzentwicklungsprozesses der Studierenden zueinander in Beziehung zu setzen und zu sequenzieren (z.B. Fragen der Integration von Praxisphasen innerhalb des Studienganges). Zur Sicherung der Qualität eines Studienganges ist (4) eine laufende Evaluation des Studienganges vorzunehmen, welche im Ergebnis zu Revisionsprozessen führt (vgl. ausführlich Gerholz \& Sloane 2016, 165ff.).

Neben der Themenstruktur sind auf organisatorischer Ebene organisatorische Elemente zu bilden, die die Aushandlungs- und Abstimmungsprozesse zwischen den beteiligten internen und externen Akteuren ermöglichen. Dabei gilt es intraorganisationale (z.B. zwischen den Disziplinen und Fächern) und interorganisationale Abstimmungen (z.B. zwischen Gesundheitseinrichtungen und Hochschule) in den Blick zu nehmen, was in Abbildung 2 exemplarisch über ,Studiengangmanagement' und ,Praxisbüros' visualisiert ist. 


\section{Mikroebene: Lehr-Lernprozessgestaltung}

Die Mikroebene zielt auf die Gestaltung der Lehr-Lernprozesse. Traditionell kann für die berufliche Bildung ein handlungsorientiertes Lern- und Didaktikverständnis festgehalten werden. Es geht um das Wechselspiel von Lernen und Arbeiten (vgl. Gerholz \& Brahm 2014, 151ff.; Czycholl 1996). Auch in den Berufsgesetzen für die Gesundheitsfachberufe spiegelt sich dieses Lernund Didaktikverständnis indirekt wider, was sich in der Differenzierung von theoretischem und praktischem Unterricht sowie der praktischen Ausbildung zeigt (vgl. PhysTh-APrV, ErgThAPrV, LogAPrO). Auf Ebene der Hochschulbildung ist demgegenüber keine einheitliche Schwerpunktsetzung zu beobachten. Dies liegt einerseits an den unterschiedlichen disziplinären $\mathrm{Zu}$ gängen innerhalb einer Hochschule sowie den damit einhergehenden didaktischen Zugängen. Andererseits spiegeln die Ordnungsgrundlagen für die Hochschulbildung (z.B. KMK-Vorgaben zur Akkreditierung von BA-/MAStudiengängen) stärker formal-strukturelle und weniger inhaltlich-didaktische Vorgaben wider (vgl. Gerholz \& Sloane 2008, 15).

In den letzten Jahren wird allerdings im hochschuldidaktischen Diskurs das Konzept des forschenden Lernens für die Hochschulbildung verstärkt diskutiert (vgl. u. a. Huber 2004, Reinmann 2009). Hierbei handelt es sich weniger um ein einheitliches Konzept (vgl. Huber 2014), sondern vielmehr um die Kontur einer Methode, deren Grundidee ein Lernen durch Forschen darstellt (vgl. Huber 2004, 32) und innerhalb der Fächer als didaktisches Gestaltungselement zu konkretisieren ist. So wird auch im Rahmen der akademischen Ausbildung in den Gesundheitsbereich ,Forschendes Lernen' v. a. in Verbindung mit dem in der beruflichen Bildung verankerten Zugang einer handlungsorientierten bzw. fallorientierten Didaktik diskutiert. Intention ist es dabei, den Lernprozess der Studierenden an didaktisch aufbereiteten Fallsituationen aus den beruflichen Handlungsfeldern des Gesundheitsbereiches zu arrangieren (vgl. u. a. Hundenborn 2006, Reiber 2012). Hierbei wäre aber stärker zu klären, inwiefern eine Konzeption des forschenden Lernens vorliegen kann, da dies Fälle implizieren würde, die in der beruflichen Praxis nicht ,gelöst' wurden, sondern vielmehr im Lernprozess erforscht werden müssten. Wird der Fokus auf die fallorientierte Didaktik gelegt, wäre näher zu ergründen, welche Fälle für eine akademisch orientierte (Aus)bildung in Betracht kommen, v. a. in Abgrenzung zu traditionell berufsfachlichen Ausbildung. Insgesamt zeigt sich in der didaktischen Intention dieser Ansätze aber eine Passung zum Leitziel der Förderung einer wissenschaftlich basierten Handlungskompetenz: Die Studierenden sollen darauf vorbereitet werden, berufliche Problemsituationen auf Basis einer wissenschaftlichen Expertise zu bewältigen und Bezüge zwischen wissenschaftlichem Wissen und berufsprakti- 
schen Erfahrungswissen aufzubauen (vgl. Schneider und Wildt 2003; Fichten o. J., 12).

Hierfür erscheint es sinnvoll, die wissenschaftliche und berufpraktische Perspektive im Studium kohärent miteinander zu verzahnen. Die Ergebnisse der Evaluation der Modellstudiengänge in Nordrhein-Westfalen zeigen dahingehend auf, dass dies vor allem in den Praxisphasen in den Gesundheitseinrichtungen noch nicht hinreichend gelingt. So berichten zum Beispiel die Pflegestudierenden, dass sie sich stärker als ,Arbeitskräfte' und weniger als Lernende in den Praxisphasen fühlen. Letzteres spiegelt nicht zuletzt die Herausforderung wider, dass sich in der Praxis die akademischen Fähigkeiten in den Gesundheitseinrichtungen (noch) nicht entfalten (vgl. Abschnitt 2). Darüber hinaus verfügen häufig die Praxisanleiter/innen in den Gesundheitseinrichtungen selber über keinen akademischen Berufsabschluss und sind nicht genügend darauf vorbereitet, die Lernprozesse entsprechend eines akademischen Profils zu begleiten (vgl. ausführlich Darmann-Finck et al. 2014, 117ff.).

Zusammenführend lassen sich somit zwei Herausforderungen beschreiben: Einerseits existiert eine Lücke zwischen dem Anspruch an hochschulische Bildungsprozesse in den Gesundheitsfachberufen und deren Realisierung in den Praxiseinrichtungen. Andererseits bedarf es einer stärkeren didaktischen Fundierung der Begleitung der Studierenden in den Praxisphasen, um diese auf ein akademisch fundiertes evidenzbasiertes Handeln vorzubereiten.

Für die skizzierten Herausforderungen liegen derzeit allerdings nur wenige Grundlagen aus einer differenzierten didaktischen Perspektive vor. Während sich die Pflegedidaktik in den vergangenen Jahrzehnten bereits mit der Entwicklung erster fachdidaktischer Ansätze etablieren konnte, besteht ein offensichtliches Desiderat bei den anderen Gesundheitsberufen wie den Gesundheitsfachberufen (vgl. Walkenhorst 2015). Um die Gestaltung der Lehr-Lernprozesse in den neu gestalteten Studiengängen adäquat umzusetzen, bedarf es eines lehrenden Personals, das in der Lage ist, die Inhalte in Theorie und Praxis akademisch anzubieten und didaktisch unterschiedliche Lehr-Lernarrangements zu gestalten, die über das bisherige berufliche Ausbildungsniveau hinausgehen. Aktuell finden sich in den Hochschulen neben den fachwissenschaftlich besetzten Professuren zumeist Lehrende, die eine einschlägige abgeschlossene berufliche Ausbildung in einem Gesundheitsberuf haben, einen akademischen Grad in einem affinen wissenschaftlichen Fach erworben haben und häufig einige Jahre als Lehrende an einer Berufsfachschule tätig waren. Ihre Lehre ist durch ein hohes inhaltliches und berufsbezogenes Engagement geprägt. Für eine fundierte akademische Lehre in den Studiengängen bedarf es jedoch der Erkenntnisse einer Didaktik der Gesundheitsfachberufe, die fachdidaktische und berufsfelddidaktische Aspekte beinhaltet. Die Lehre in den Gesundheitsfachberufen weist dennoch 
seit vielen Jahren einen hohen Innovationsgrad aus, der durch verschiedene Reformen unterstützt wurde (vgl. Klemme 2012). Dieses Potenzial kann für den notwendigen Entwicklungsstrang einer Fachdidaktik im Rahmen des Akademisierungsprozesses genutzt und ausgebaut werden.

\section{Fazit}

Der vorliegende Aufsatz hatte zum Ziel, den Stand der Umsetzung der Akademisierung der Gesundheitsfachberufe zu beleuchten. Dabei wurden über die Handlungsebenen Herausforderungen und Gestaltungsansätze skizziert, die in Tabelle 1 zusammengeführt sind. Die herausgearbeiteten Herausforderungen zeigen den Bedarf an weiteren Entwicklungen in der Akademisierung der Gesundheitsfachberufe auf. Die aufgezeigten Gestaltungsansätze können dabei Orientierungspunkte für die Weiterentwicklung darstellen. Dabei spiegeln diese gleichzeitig Forschungsdesiderate wider, die nachfolgend für die Handlungsebenen skizziert werden.

\section{Tab. 1: Akademisierung der Gesundheitsfachberufe: Herausforderungen und Gestaltungsmöglichkeiten}

\begin{tabular}{lll}
\hline Ebene & Herausforderungen & Gestaltungsansätze \\
\hline $\begin{array}{l}\text { Makro- } \\
\text { ebene }\end{array}$ & $\begin{array}{l}\text { Herausforderung 1: Durch Beibehal- } \\
\text { tung der Berufsgesetze nur formale } \\
\text { Anhebung der Ausbildungsvoraus- } \\
\text { setzungen. }\end{array}$ & $\begin{array}{l}\text { Möglichkeit der Etablierung } \\
\text { korporatistischer Strukturen }\end{array}$ \\
& $\begin{array}{l}\text { Herausforderung 2: Gefahr der } \\
\text { Dequalifizierung des Bachelorab- } \\
\text { schlusses, da fehlende akademische }\end{array}$ & $\begin{array}{l}\text { Definition von Tätigkeits- und } \\
\text { Berufs(feld)profilen in Verant- } \\
\text { wortung der Interessensgruppen } \\
\text { tem. }\end{array}$ \\
& $\begin{array}{l}\text { Herausforderung 3: Berufsgesetze } \\
\text { spiegeln nicht die akademischen }\end{array}$ & $\begin{array}{l}\text { Anpassung der Berufsgesetze auf } \\
\text { akademische Profile }\end{array}$ \\
& Anforderungen wider. & \\
\hline
\end{tabular}


Tab. 1: Akademisierung der Gesundheitsfachberufe: Herausforderungen und Gestaltungsmöglichkeiten (Fortsetzung)

\begin{tabular}{|c|c|c|}
\hline Ebene & Herausforderungen & Gestaltungsansätze \\
\hline \multirow[t]{3}{*}{$\begin{array}{l}\text { Meso- } \\
\text { ebene }\end{array}$} & $\begin{array}{l}\text { Herausforderung 1: Passungs- } \\
\text { probleme zwischen Bildungszielen }\end{array}$ & $\begin{array}{l}\text { Modell der diskursiven Studien- } \\
\text { gangentwicklung: }\end{array}$ \\
\hline & $\begin{array}{l}\text { und curricularem Design auf Studi- } \\
\text { engangebene. }\end{array}$ & $\begin{array}{l}\text { Prozessuale Ebene: Themen- } \\
\text { struktur zur Entwicklung eines } \\
\text { kohärenten Curriculums }\end{array}$ \\
\hline & $\begin{array}{l}\text { Herausforderung } 2 \text { : Koordination und } \\
\text { Verankerung intra- und interorganisa- } \\
\text { tionale Abstimmungsprozesse }\end{array}$ & $\begin{array}{l}\text { Organisatorische Ebene: organi- } \\
\text { satorische Äquivalente zur Ein- } \\
\text { bindung der Bezugsdisziplinen } \\
\text { und Praxiseinrichtungen }\end{array}$ \\
\hline \multirow[t]{2}{*}{$\begin{array}{l}\text { Mikro- } \\
\text { ebene }\end{array}$} & $\begin{array}{l}\text { Herausforderung 1: Praxisphasen } \\
\text { ermöglichen nur bedingt akademi- } \\
\text { sche Kompetenzentwicklung }\end{array}$ & $\begin{array}{l}\text { Entwicklung einer Fachdidaktik } \\
\text { für eine akademisierte Ausbil- } \\
\text { dung in den Gesundheitsfachbe- } \\
\text { rufen }\end{array}$ \\
\hline & $\begin{array}{l}\text { Herausforderung 2: Fehlende Beglei- } \\
\text { tungskonzepte zur Förderung eines } \\
\text { evidenzbasierten Handelns }\end{array}$ & $\begin{array}{l}\text { Qualifizierung der Praxisanleiter } \\
\text { zur Förderung eines evidenzba- } \\
\text { sierten Handelns }\end{array}$ \\
\hline
\end{tabular}

Auf der Makroebene ergibt sich das Desiderat, wie aus Steuerungsperspektive eine stärkere Verzahnung der einzelnen Interessensgruppen (Beschäftigungssystem, Bildungssystem und Bildungspolitik) organisiert werden kann. Für die Möglichkeit der Etablierung korporatistischer Strukturen ist näher zu untersuchen, unter welchen Bedingungen diese eine steuernde Wirkung für die Intention der Akademisierung der Gesundheitsfachberufe entfalten können. So sind Hochschulen in ihrem Selbstverständnis durch eine hohe Autonomie geprägt, die nicht zuletzt ein gesellschaftliches Interesse widerspiegelt, aber die Etablierung von korporativen Strukturen erschweren kann. Weiterhin wären die Akteure auf Ebene des Beschäftigungssystems näher zu spezifizieren. So existiert seit Jahren eine Diskussion über die Gründung einer ,Pflegekammer' als Pendant zu den Ärztekammern. Hier stellt sich das Problem der Spezifizierung der Arbeitgeberseite. Dies kann nicht zuletzt Einflüsse darauf haben, welche Vorstellungen bei Berufsfeldprofilen in den Gesundheitsfachberufen sich etablieren.

Auf der Mesoebene ist zukünftig näher zu beobachten, wie sich das Phänomen der ,Gesundheitswissenschaften' organisatorisch und disziplinär innerhalb der Hochschulstruktur entwickelt und verankert (z.B. eigene Fachbereiche vs. Verankerung innerhalb medizinischer Fachbereiche). Es können Wechselwirkungen zwischen den vorhandenen Traditionen etablierter Fachbereiche und die diskursive Aushandlung eines kohärenten curricularen Designs angenommen werden. Gleichzeitig sind Hochschulen aufgrund der aktuell existierenden Berufsgesetze angewiesen, praktische (Aus)bildungs- 
anteile in den Studiengänge zu integrieren, was Abstimmungsleistungen zwischen Hochschulen und Praxiseinrichtungen erfordert. Die Möglichkeiten der Organisation dieser Abstimmungsleistungen und der Qualitätssicherung des intendierten akademisch orientierten Kompetenzentwicklungsprozesses bei den Studierenden sind näher zu eruieren. Dabei geht es nicht zuletzt auch um die Frage, welche Kompetenzstrukturen - v. a. in Abgrenzung zu der berufsfachlichen Ausbildung - ein akademisches Profil bestimmen. Hier wäre die Anbindung an den internationalen Diskurs vorzunehmen. So kann das CanMeds-Modell zur Kompetenzmodellierung in der Medizinerausbildung (vgl. Frank 2005) Anschlüsse für die Gesundheitsfachberufe aufzeigen.

Auf der Mikroebene geht es um die Entwicklung und Etablierung einer Fachdidaktik für eine akademisierte (Aus)bildung in den Gesundheitsfachberufen. Die Zuschneidung einer solchen Fachdidaktik ist innerhalb der Community und mit den relevanten Stakeholdern auszuhandeln. Hierbei kann die Anbindung - v. a. in Hinblick auf die Lehr-Lernforschung - an den internationalen Diskurs relevante Akzente für die (Weiter)Entwicklung einer solchen Fachdidaktik bieten. Betrachtet man die Rahmenbedingungen der Akademisierung wie die didaktische Gestaltung von Praxisphasen, erscheint eine Anbindung an den Diskurs des ,Practice Based Learning' (vgl. dazu u. a. Billet 2015) sinnvoll bzw. aus einer stärker fachdidaktischen Perspektive eine Anbindung an den Diskurs ,Nursing Education' u. a. in Hinblick auf die Gestaltung von Lernumgebungen für die Förderung evidenzbasierten Handelns (vgl. dazu u. a. Robinson \& Dearmon 2013). Der internationale Diskurs kann gleichermaßen Orientierungspunkte für die Professionalisierung der Praxisanleiter/innen (,precpetor research') bieten, u. a. inwiefern assessmentorientierte Verfahren Praxisanleiter/innen in der Förderung eines akademisch orientierten Kompetenzprofils der Studierenden unterstützen können (vgl. dazu Wu, Enskär, Lee \& Wang 2015).

\section{Literatur}

AGBB - Autorengruppe Bildungsberichterstattung (2015). Bildung in Deutschland 2015. Bielefeld: W. Bertelsmann.

Baethge, M. \& Wolter, Ä. (2015). The German skill formation model in transition: from dual system of VET to higher education? J Labour Market Res, 48(2), 97112.

Bourgeault, I., Kuhlmann, E., Neitermann, E. \& Wrede, S. (2008). Wie kann ein optimaler Qualifikationsmix effektiv verwirklicht werden - und warum? Grundsatzpapier WHO. Weltgesundheitsorganisation im Namen des Europäischen Observatoriums für Gesundheitssysteme und Gesundheitspolitik.

Cornbleth, C. (1990). Curriculum in context. London and New York: Falmer. 
Czycholl, R. (1996). Handlungsorientierung in der beruflichen Bildung. In B. Bonz (Hrsg.), Didaktik der Berufsbildung. Beiträge zur Pädagogik für Schule und Betrieb, (S. 113-131). Stuttgart: Schneider Hohengehren.

Darmann-Finck I., Muths, S., Görres, S., Adrian C., Bomball, J. \& Reuschenbach B. (2014). Inhaltliche und strukturelle Evaluation der Modellstudiengänge zur Weiterentwicklung der Pflege- und Gesundheitsfachberufe in NRW. Abschlussbericht. Online: http://www.mgepa.nrw.de/mediapool/pdf/pflege/ 20150528_NRWAbschlussbericht-End-26_05_2015.pdf (12.02.2016).

Euler, D. (2014). Berufs- und Hochschulbildung - (Ungleicher) Wettbewerb oder neue Formen des Zusammenwirkens? Zeitschrift für Berufs- und Wirtschaftspädagogik, 110(3), 321-334.

Fichten, W. (o.J.). Forschendes Lernen in der Lehrerbildung. Online: http://www.unioldenburg.de/fileadmin/user_upload/paedagogik/as/forschungswerkstatt/ download/Forschendes_Lernen.pdf (23.12.2015).

Frank, J. R. (2005). The CanMEDS 2005 physician competency framework. Better standards. Better physicians. Ottawa, Canada: The Royal College of Physicians and Surgeons of Canada. Online: http://www.ub.edu /medicina_unitateducaciomedica/documentos/CanMeds.pdf (23.03.2016).

GEBK (2009). Gesetz über die Einführung einer Modellklausel in die Berufsgesetze der Hebammen, Logopäden, Physiotherapeuten und Ergotherapeuten vom 25. September 2009, verkündet in BGBl I Jahrgang 2009 Nr. 64 vom 2.10.2009.

Gerholz, K.-H. (2013). Akademisierung der Gesundheitsfachberufe - Curriculare und didaktische Potentiale entfalten. Therapie Lernen. Zeitschrift für Lehrende und Lernende, 2, 16-23.

Gerholz, K.-H. \& Brahm, T. (2014). Apprenticeship and Vocational Education. In C. Harteis, A. Rausch \& J. Seifried (Hrsg.): Discourses on professional learning: On the boundary between learning and working, (S. 143-158). Dordrecht: Springer.

Gerholz, K.-H. \& Sloane, P.F.E. (2016). Diskursive Studiengangentwicklung. In T. Brahm, T. Jenert \& D. Euler (Hrsg.): Pädagogische Hochschulentwicklung: von der Programmatik zur Implementierung, (S. 151-170). Wiesbaden et al.: Springer.

Gerholz, K.-H. \& Sloane, P. F. E. (2011). Lernfelder als universitäres Curriculum? Eine hochschuldidaktische Adaption. bwp@Berufs- und Wirtschaftspädagogikonline, 20, 1-24. Online: http://www.bwpat.de/ausgabe20/gerholz _sloane_bwpat 20.pdf (01.06.2016).

Gerholz, K.-H. \& Sloane, P. F. E. (2008). Der Bolognaprozess aus curricularer und hochschuldidaktischer Perspektive - Eine Kontrastierung von beruflicher Bildung und Hochschulbildung auf der Bachelorstufe. bwp@Berufs- und Wirtschaftspädagogik - online, 14, 1-22. Online: http://www.bwpat.de/ausgabe14/ gerholz_sloane_bwpat14.pdf (01.06.2016)

Huber, L. (2004). Forschendes Lernen - 10 Thesen zum Verhältnis von Forschung und Lehre aus der Perspektive des Studiums. Die Hochschule, 2, 29-49.

Huber, L. (2014). Forschungsbasiertes, Forschungsorientiertes, Forschendes Lernen: Alles dasselbe? Ein Plädoyer für eine Verständigung über Begriffe und Unterscheidungen im Feld forschungsnahen Lehrens und Lernens. Das Hochschulwesen, 62(1+2), 22-29. 
Hundenborn, G. (2006). Fallorientierte Didaktik in der Pflege: Grundlagen und Beispiele für Ausbildung und Prüfung. München, Jena: Urban \& Fischer.

Klemme, B. (Hrsg.) (2012). Lehren und Lernen in der Physiotherapie. Stuttgart: Thieme Verlag.

KMK - Sekretariat der Ständigen Konferenz der Kultusminister der Länder in der Bundesrepublik Deutschland (2000). Handreichung für die Erarbeitung von Rahmenlehrplänen der Kultusministerkonferenz für den berufsbezogenen Unterricht in der Berufsschule und ihre Abstimmung mit Ausbildungsordnungen des Bundes für anerkannte Ausbildungsberufe. Online: http://www.kmk.org/doc/ publ/handreich.pdf (17.08.2011).

KrPflAPrV - Ausbildungs- und Prüfungsverordnung für die Berufe in der Krankenpflege. Online: http://www.gesetze-im-internet.de/krpflaprv_2004/ (03.10.2015).

LPPhyBY (2013). Lehrplan für die Berufsfachschule für Physiotherapie in Bayern. Online:https://www.isb.bayern.de/download/13777/lp_bfs_physio_sept_2013.pdf (04.04.2016).

LPPhyNRW (2005). Empfehlende Ausbildungsrichtlinie für staatliche anerkannte Physiotherapieschulen in Nordrhein-Westfalen. Online: http://www.mgepa.nrw. de/mediapool/pdf/pflege/pflege_und_gesundheitsberufe/ausbildungsrichtlinien/a usbildungsrichtlinien-physiotherapieschulen-nrw barr.pdf (02.03.2016).

LPErgB (2003). Berliner Lehrplan für die Ergotherapieausbildung. Online: https://www.berlin.de/sen/gesundheit/_../lehrplan_ergotherapie.pdf (03.04.2016)

PhysTh-APrV - Ausbildungs- und Prüfungsverordnung für Physiotherapeuten. Online: http://www.gesetze-im-internet.de/physth-aprv/BJNR378600994.html (03.10. 2015).

Reinmann, G. (2009). Wie praktisch ist die Universität? Vom situierten zum forschenden Lernen mit digitalen Medien. Online: http://gabi-reinmann.de/wpcontent/uploads/2009/08/Artikel_Forschendes_situiertes_Lernen09.pdf (04.05. 2013).

Reiber, K. (2012). Hochschuldidaktik für gesundheitsbezogene Studiengänge. Eine theoretische Grundlegung. Tübinger Beiträge zur Hochschuldidaktik, Band 8/1. Online: http://nbn-resolving.de/urn:nbn:de:bsz:21-opus-62906 (04.08.2015).

Robinson, B.K. \& Dearmon, V. (2013). Evidence-based nursing education: effective use of instructional design and simulated learning environments to enhance knowledge transfer in undergraduate nursing students. Journal Professional Nursing, 29 (1), 203-209.

Schneider, R. \& Wildt, J. (2003). Das Berufspraktische Halbjahr in Dortmund: Forschendes Lernen in Praxisstudien einer professionalisierten Lehrerausbildung. In A. Obolenski \& H. Meyer (Hrsg.), Forschendes Lernen. Theorie und Praxis einer professionellen LehrerInnenausbildung, (S. 165-183). Bad Heilbrunn: Klinkhardt.

Schön, D. A. (1984). The Reflective Practitioner: How Professionals Think in Action. New York: basic books.

Sottas, B., Höppner, H., Kickbusch, I., Pelikan, J. \& Probst, J. (2013). Umrisse einer neuen Gesundheitsbildungspolitik. Careum working paper 7. Zürich.

Walkenhorst, U. (2015). Gesundheitsberufe zwischen beruflicher und hochschulischer Bildung - Potenziale, Paradoxien und Perspektiven. In A. Beaugrand (Hrsg.). Bildung anführen. Über Hochschulmanagement nach der Bologna-Reform, (S. 278-299). Bielefeld: Transcript Verlag. 
Walkenhorst, U. (2011). Akademisierung der therapeutischen Gesundheitsfachberufe - Chancen und Herausforderungen für Berufe im Übergang. bwp@ Spezial 5 Hochschultage Berufliche Bildung 2011, Fachtagung 10, hrsg. v. BonseRohmann, M. \& Weyland, U., 1-12. Online: http://www.bwpat.de/ht2011/ft10/ walkenhorst_ft10-ht2011.pdf (16.07.2015).

Walkenhorst, U. (2008). Potenziale der Ergotherapie in der Gesundheits- und Krankenversorgung. Eine handlungsorientierte professionssoziologische Analyse. Idstein: Schulz-Kirchner Verlag.

Wolter, A. (2015). Hochschulexpansion: Wachsende Teilhabe oder Akademisierungswahn? Online: http://www.bpb.de/gesellschaft/kultur/zukunft-bildung/200 104/teilhabe-oder-akademisierungswahn (01.09.2015)

WR (Wissenschaftsrat) (2012). Empfehlungen zu hochschulischen Qualifikationen für das Gesundheitswesen. Online: http://www.wissenschaftsrat.de/download/archiv/ 2411-12.pdf (03.08.2015)

Wu, X. V., Enskär, K., Lee, C. C. S. \& Wang, W. (2015). A systematic review of clinical assessment for undergraduate nursing students. Nurse Education Today, 35(2), 347-359. 



\title{
Handeln in Geschäftsprozessen als Forschungs- und Lehr-Lern-Gegenstand
}

\author{
Juliana Schlicht
}

\section{Geschäftsprozessorientierung in der beruflichen Aus- und Weiterbildung - Ausgangslage und Handlungsbedarf}

Geschäftsprozessorientierung gilt seit den 1990er Jahren als zentrales Prinzip der curricularen und didaktisch-methodischen Ausgestaltung lernfeldstrukturierter Curricula - sowohl in der kaufmännischen als auch in der gewerblichtechnischen Berufsausbildung ${ }^{1}$ (vgl. Busian 2011; Reinisch 2014; Tramm 2009). Darüber hinaus wird auch im Bereich der beruflichen Weiterbildung diskutiert, wie das Lernen und Lehren geschäftsprozessorientiert auszugestalten ist (vgl. Baethge \& Baethge-Kinsky 2004, 22).

Am Beispiel des neu geordneten Berufs Kaufmann/-frau für Büromanagement wird deutlich, dass der Anspruch an die Geschäftsprozessorientierung derzeit - zumindest curricular - eine neue "Qualität“ erreicht hat (vgl. Harms 2014). Es geht in den Lehrplänen darum, dass die Auszubildenden die Kompetenz erwerben sollen, Geschäftsprozesse zu analysieren, modellhaft abzubilden, zu reflektieren und zu optimieren. Darüber hinaus sollen sie in der Lage sein, in Geschäftsprozessen effektiv zu handeln - das heißt v. a., zielgerichtet und zweckmäßig zu kommunizieren und zu kooperieren.

Es stellt sich die Frage, inwieweit dieser Anspruch in der Aus- und Weiterbildung erfüllt werden kann, und zwar aus folgenden Gründen: Zum einen liegen bisher kaum empirisch gesicherte, berufs- und wirtschaftspädagogisch verwertbare Erkenntnisse darüber vor, wie die Kommunikation und Kooperation von Fach- und Führungskräften in den Geschäftsprozessen im Unternehmen tatsächlich verlaufen (vgl. Achtenhagen \& Winther 2011) und welche kognitiven, motivationalen sowie emotionalen Handlungsdispositionen dafür erforderlich sind (vgl. Wuttke, Seifried, Brandt, Rausch, Sembill, Martens \& Wolf 2015). Die Studien konzentrieren sich bisher vor allem auf einzelne kommunikative Handlungsakte (z.B. die adressatengerechte Nutzung von Informations- und Kommunikationsmedien), die eine Person bei der Bewältigung berufstypischer, in Geschäftsprozessen verorteter Arbeitssitua-

1 Das Prinzip wurde erstmals bei den IT-Berufen (Informatikkaufmann/-frau; Informationsund Telekommunikationssystem-Kaufmann/-frau; Fachinformatiker/-in; Informations- und Telekommunikationssystem-Elektroniker/-in) curricular verankert (vgl. Borch, Ehrke, Müller \& Schwarz 1999). 
tionen vollzieht (vgl. u. a. Achtenhagen \& Winther 2011). Wechselseitige Interaktionsprozesse von zwei und mehr Personen, die das Kommunizieren und Kooperieren in Geschäftsprozessen wesentlich bestimmen, wurden bislang vor allem aufgrund methodischer Defizite nicht untersucht (vgl. Achtenhagen \& Winther 2009, 14; Nickolaus 2015, 163).

Zum anderen wird in den Rahmenlehrplänen und einschlägigen Schulund Lehrbüchern (vgl. u. a. Cersovsky, Hunold \& Squarra 2007; Tramm, Adler, Frost, Goldbach, Seidler \& Wichmann 2007; Wilbers 2012) zur Veranschaulichung der Arbeitsabläufe in Geschäftsprozessen bisher weitgehend unreflektiert auf Konzepte und Verfahren der Betriebswirtschaftslehre (BWL) und der Wirtschaftsinformatik (Winf) zurückgegriffen, die sowohl aus fachwissenschaftlicher Sicht als auch aus berufs- und wirtschaftspädagogischer Perspektive defizitär sind, vor allem weil sie die zwischenmenschliche Kommunikation und Kooperation weitgehend außer Acht lassen.

Zudem liegt der Fokus der Diskussion im Fach um die thematische Ausrichtung kaufmännischer Aus- und Weiterbildung bisher vor allem auf der Frage, wie betriebliche Güter- und Leistungsströme, Geld- und Wertströme sowie Informationsströme und Daten (auf der Dokumenten- und Belegebene) curricular und didaktisch-methodisch aufbereitet werden können (vgl. Achtenhagen, John, Preiß, Tramm, Seemann-Weymar \& Schunck 1992, 111; Busian 2011, 5; Getsch \& Preiß 2003, 19; Tramm 2009, 84).

Um dem im Fach formulierten Anspruch eines ausbalancierten Wissenschafts-, Situations- und Persönlichkeitsbezugs (vgl. Tramm \& Reetz 2010) in der geschäftsprozessorientierten Aus- und Weiterbildung gerecht werden zu können, ist es aus Sicht der Autorin notwendig, güter- und leistungsbezogene, finanzielle und informationstechnische Tätigkeitsaspekte stärker als bisher mit sozialen und emotionalen Bedingungsfaktoren des Handelns in Geschäftsprozessen thematisch zu verknüpfen. Dafür sind zum einen fachdidaktische Fragen zur Inhaltsauswahl, Sequenzierung und Komplexitätsgestaltung zu bearbeiten (vgl. Reinisch 2014). Zum anderen ist dem Defizit an empirisch fundierten Erkenntnissen zur Kommunikation und Kooperation in Geschäftsprozessen entgegenzuarbeiten.

Der vorliegende Beitrag greift diese Problematik im Kontext einer Studie zur Analyse und Neugestaltung von Geschäftsprozessen in einem Unternehmen der Energiewirtschaft auf. Es wird zunächst das zugrunde liegende Begriffsverständnis erläutert. Anschließend werden die Grenzen der fachwissenschaftlichen (BWL, Winf) Ansätze und Methoden in Bezug auf die Analyse und Modellierung geschäftsprozessbezogener Kommunikation und Kooperation erörtert. Kontrastierend dazu werden ein wirtschaftspädagogischer Untersuchungsansatz für Geschäftsprozesse im Unternehmen sowie einige Befunde einschließlich deren Nutzung für Curriculumentwicklung und LehrLern-Prozessgestaltung skizziert. 


\section{Begriffsverständnis: Geschäftsprozess}

Der Geschäftsprozess ist ein genuin betriebswirtschaftliches Konstrukt (vgl. u. a. Becker \& Kahn 2012; Gaitanides 2012), das auch in der Wirtschaftsinformatik genutzt wird, um Arbeitsaufgaben und Arbeitsabläufe zu beschreiben und zu strukturieren (vgl. u. a. Fleischmann, Schmidt, Stary, Obermeier \& Börger 2011; Scheer \& Thomas 2009). Geschäftsprozess wird definiert als eine Summe von miteinander verknüpften Aktivitäten (Aufgaben), die zum einen von Menschen verschiedener Organisationsbereiche in sachlogischer und zeitlicher Reihenfolge mit Hilfsmitteln zur Bearbeitung eines Geschäftsobjekts ausgeführt werden, um u. a. ein Kundenbedürfnis zu befriedigen und zur Wertschöpfung beizutragen; und die zum anderen einen definierten Anfang und Input sowie ein definiertes Ende und Ergebnis aufweisen (Fleischmann et al. 2011, 42). Dieses Begriffsverständnis dominiert auch in der Ausund Weiterbildung (vgl. Rebmann \& Schlömer 2009, 3; Tramm 2004, 137).

Folgt man der Argumentation von Reinisch (2012; 2014), kann das betriebswirtschaftliche und wirtschaftsinformatische Verständnis allerdings nur bedingt Grundlage geschäftsprozessorientierter Curricula sein, vor allem weil die Konzepte und Verfahren der Betriebswirtschaftslehre, Geschäftsprozesse aus Managementperspektive thematisieren, wobei sie die kommunizierenden und kooperierenden Subjekte weitgehend außer Acht lassen. Bisher liegen zudem kaum empirisch gesicherte, berufs- und wirtschaftspädagogisch verwertbare Erkenntnisse über die konkrete Kommunikation und Kooperation in einzelnen betrieblichen Geschäftsprozessen einschließlich der Bedingungsfaktoren dafür vor. ${ }^{2}$ Deshalb ist es erforderlich, einen Ansatz zu entwickeln, der bezogen auf konkrete Geschäftsprozesse in der Unternehmung das ,handelnde Subjekt, dessen Aufgaben und deren wahrscheinliche Entwicklung, dessen Kooperations- und Interaktions- sowie Konfliktverhalten, dessen Arbeitsbedingungen und gesellschaftliche Stellung in den Mittelpunkt der Betrachtung stellt, um daraus die in der Berufsausbildung zu erwerbenden Kenntnisse, Fähigkeiten und Einstellungen zu gewinnen“" (vgl. Reinisch 2012, 7).

Zwar ist auch den Betriebswirten und den Wirtschaftsinformatikern bewusst, dass Kommunikation und Kooperation zentrale Komponenten des

2 Das liegt auch daran, dass im Fach zuweilen die These vertreten wird, betriebliche Geschäftsprozesse ließen sich empirisch nicht fassen (Tramm 2004, 138) und die Fachwissenschaften (BWL, Winf) böten „den besten Zugang zu den beruflichen Handlungssituationen“ (vgl. Getsch \& Preiß 2003, 3). Hinzu kommt, dass kommunikationswissenschaftliche und sozialpsychologische Definitions- und Untersuchungsansätze im Fach bisher lediglich vor allem genutzt wurden, um die Lehrer-Schüler-Interaktion zu beschreiben und zu erforschen (vgl. Euler \& Hahn 2007, 397-463; Wuttke 2005). Bezogen auf Geschäftsprozesse in den Unternehmen und für die Geschäftsprozessorientierung in der Aus- und Weiterbildung werden solche Ansätze bisher kaum genutzt. 
Denkens und Handelns in Geschäftsprozessen sind. So betont bspw. Gaitanides (2012, 102), dass das Konstrukt „Geschäftsprozess“ erst verwirklicht wird, wenn Menschen zur Erledigung ihrer Arbeitsaufgaben miteinander kommunizieren und kooperieren. Fleischmann et al. (2011) konstatieren zudem, dass Kommunikation und Kooperation auch bei der Softwareentwicklung berücksichtigt werden müssen. Allerdings gelingt es beiden Disziplinen bisher kaum, zwischenmenschliche Kommunikation und Kooperation in Geschäftsprozessmodellen adäquat abzubilden (vgl. Schmelzer \& Sesselmann 2013, 658; Weber, Schmidt \& Weber 2012, 210). Das liegt vor allem daran, dass Kommunikation und Kooperation komplexe soziale Phänomene des wechselseitigen Informationsaustauschs und der wechselseitigen Bedeutungsvermittlung sind (vgl. Burkart 2002, 61), die sich den linearen betriebswirtschaftlichen und wirtschaftsinformatischen Abbildungsformen weitgehend entziehen. Darauf wird im Folgenden näher eingegangen.

\section{Ansätze der Betriebswirtschaftslehre und Wirtschaftsinformatik}

Um Arbeitsaufgaben sowie zugehörige Daten- und Informationsflüsse in einer sachlogischen und zeitbezogenen Reihenfolge zu modellieren, werden in den Fachwissenschaften (BWL, Winf) und in den Unternehmen bisher vor allem gerichtete, relativ lineare Abbildungsformen präferiert, wie z.B. Flussdiagramme sowie einfache und erweiterte ereignisgesteuerte Prozessketten ${ }^{3}$ (vgl. Gadatsch 2012; Scheer \& Thomas 2009; Schmelzer \& Sesselmann 2013, 474). Die organisatorische und technische Modellierung erfolgt dabei auf der Grundlage einer spezifischen Symbolik, wie das folgende Beispiel der Beschwerdebearbeitung zeigt (vgl. Abbildung 1):

3 Eine ereignisgesteuerte Prozesskette ist ein wirtschaftsinformatisches Konzept. Darunter wird ,ein gerichteter und zusammenhängender Graph [gefasst], dessen Knoten Ereignisse, Funktionen und Verknüpfungsoperatoren sind“ (vgl. Scheer \& Thomas 2009, 551) und mit dessen Hilfe betriebliche Abläufe beschrieben und analysiert werden können (vgl. Spath 2009, 5). Sogenannte erweiterte ereignisgesteuerte Prozessketten enthalten zudem Symbole für Organisationseinheiten (z.B. Abteilungen) sowie Informationsmedien (vgl. Schmidt, Fleischmann \& Gilbert 2009, 55). Darüber hinaus gibt es weitere grafische Formen sowie Modellierungssprachen, die in den Fachwissenschaften und in der betrieblichen Praxis zur informationstechnischen Abbildung von Geschäftsprozessen genutzt werden, z.B. das Entity Relationship Model (ERM), das semantische Objektmodell (SOM), die Unified Modeling Language (UML) und die Business Process Modeling Notation (BPMN) (vgl. Scheer \& Thomas 2009; Schmidt et al. 2009, 55). 
Abb. 1: Flussdiagramm und erweiterte ereignisgesteuerte Prozesskette (Beispielsymbolik)

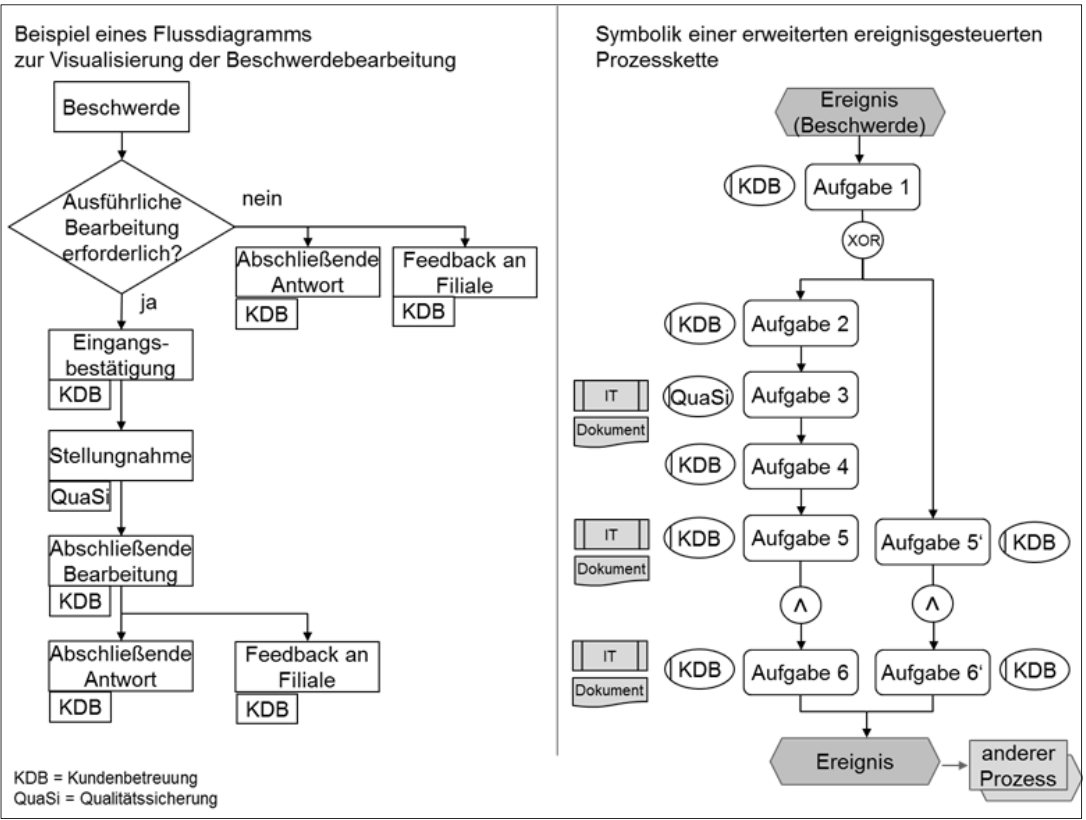

Quelle: Eigene Darstellung in Anlehnung an (Stauss \& Seidel 2014, 176) und (Gadatsch 2012, 80).

Während Flussdiagramme darauf gerichtet sind, Ja-Nein-Entscheidungen, Aufgaben und zugehörige Informationsflüsse zwischen Organisationseinheiten (z.B. Abteilungen) symbolisch abzubilden, sind in erweiterten ereignisgesteuerten Prozessketten zudem Informationsmedien angegeben sowie Bezüge $\mathrm{zu}$ anderen Prozessen mithilfe mathematisch-logischer Operatoren modelliert (vgl. Scheer \& Thomas 2009, 556). Beide Abbildungsformen beschreiben und strukturieren die Arbeitsabläufe und Informationsflüsse zum einen in Form einer Reiz-Reaktionsfunktion. Zum anderen abstrahieren die Abbildungsformen insbesondere von sozialen, aber auch von motivationalen und emotionalen Aspekten des Handelns. Der Fokus liegt vielmehr auf betriebswirtschaftlicher und informationstechnischer Zweckmäßigkeit sowie auf den ökonomischen Ergebnissen des Handelns im Geschäftsprozess. Das heißt, Flussdiagramme und erweiterte ereignisgesteuerte Prozessketten werden in den Fachwissenschaften vor allem genutzt, um Informationen zu modellieren und Software zu entwickeln (vgl. Scheer \& Thomas 2009; Staud 2006, 311). 
Im Zentrum der Informationsmodellierung stehen dabei Objekte, wie Ereignisse, Arbeitsaufgaben sowie „menschliche und maschinelle Komponenten (Teilsysteme) als Aufgabenträger" (vgl. Scheer \& Thomas 2009, 546). Der Mensch wird hier eher als eine Systemkomponente betrachtet, dessen Verhalten (im Sinne beobachtbarer Aufgabenbewältigung) relativ linear sequenziert und entsprechend vorgegebener Modelle verläuft.

In der Wirtschaftsinformatik wird zwar zunehmend auch mit sogenannten „subjektorientierten“ Ansätzen gearbeitet (vgl. Fleischmann et al. 2011). Allerdings liegt diesen Ansätzen ein Subjektbegriff zugrunde, der sich vom epistemologischen Subjektverständnis der Berufs- und Wirtschaftspädagogik (vgl. Groeben \& Scheele 1977) deutlich unterscheidet. Aus wirtschaftsinformatischer Sicht können z.B. auch Drucker oder Computer Subjekte der Kommunikation und Kooperation sein. Darüber hinaus verfügen auch die subjektorientierten Ansätze kaum über geeignete Methoden und Instrumente zur Analyse oder Optimierung geschäftsprozessbezogener zwischenmenschlicher Kommunikation und Kooperation (vgl. Weber et al. 2012, 210).

Das Problem besteht darin, dass die Berufs- und Wirtschaftspädagogik in der Diskussion um Geschäftsprozessorientierung vor allem auf diese Ansätze der BWL und Winf zumeist „relativ unreflektiert“ zurückgreift. Das heißt, dass die Prämissen und Grenzen der fachwissenschaftlichen Modellierungen im Fach zwar diskutiert werden (vgl. Busian 2011, 5; Tramm 2009), insbesondere aber deren Defizite in Bezug auf die Analyse und Abbildung zwischenmenschlicher Kommunikation und Kooperation bisher kaum untersucht und zum Lehr-Lern-Gegenstand gemacht werden. Darüber hinaus fehlt bisher ein genuin berufs- und wirtschaftspädagogischer Ansatz zur Analyse und Modellierung von Geschäftsprozessen, der die fachwissenschaftlichen Ansätze nutzt und zugleich deren Defizite überwindet. Ein solcher wird im Folgenden zur Diskussion gestellt.

\section{Analyse und Modellierung von Geschäftsprozessen in einem Unternehmen der Energiewirtschaft}

\subsection{Untersuchungsdesign}

Am Leipziger Institut für Wirtschaftspädagogik wurden in einem Projekt mit einem Unternehmen der Energiewirtschaft die Kommunikation und Kooperation in unterschiedlichen Geschäftsprozessen untersucht (vgl. Schlicht \& 
Klauser 2014) ${ }^{4}$. Um einerseits die Komplexität der Kommunikation und Kooperation empirisch analysieren zu können und um andererseits fachdidaktische Erkenntnisse für die Ausgestaltung geschäftsprozessbezogener Inhalte generieren zu können, wurden aus insgesamt 37 unternehmensspezifischen Geschäftsprozessen fünf Prozesse (zur Personalplanung, zur Personalentwicklung, zum Regulierungsmanagement, zur Auftragsbearbeitung Biogas sowie zum Kundenzufriedenheitsmanagement) ausgewählt, die folgende relevanz- und komplexitätsbestimmenden Kriterien erfüllen: ${ }^{5}$

- Vernetztheit: Am Prozess sind potenziell viele Personen beteiligt, die mehrere thematisch zusammenhängende Aufgaben bearbeiten.

- Polythelie: Mit dem Prozess werden mehrere Ziele verfolgt, die aus Sicht der beteiligten Personen gegenläufig wirken können.

- Dynamik und Intransparenz: Das Kommunizieren und Kooperieren im Geschäftsprozess wird unmittelbar durch soziale, güter- und leistungsbezogene materielle, finanzielle, rechtliche, technische und ökologische Bedingungen des Wirtschaftens im Energiebereich determiniert, die nicht jede der am Prozess beteiligten Personen gleichermaßen kennen muss bzw. zu denen Informationen nicht unmittelbar zugänglich sind und die sich potenziell kurz- und mittelfristig verändern.

- $\quad$ Thematische Breite und Interdisziplinarität: Es werden sowohl Kern- als auch Unterstützungsprozesse untersucht. Das Aufgabenspektrum eines Prozesses bezieht sich dabei auf mehrere Wertschöpfungsstufen und erfordert die Zusammenarbeit sowie Kommunikation und Kooperation von Mitarbeitern aller Organisationsbereiche.

- Subjektive Bedeutsamkeit: Die im Prozess ausgetauschten Informationen sind aus Sicht der Führungskräfte und Mitarbeiter für ihre individuelle Entwicklung und für die Bearbeitung aktueller und künftiger betrieblicher Problemstellungen bedeutsam.

4 Die Untersuchung erstreckte sich auf den Zeitraum von Mai 2012 bis April 2014. Dabei wurde ein analytisch-konstruktiver Ansatz zur Modellierung von Kommunikation und Kooperation in Geschäftsprozessen verfolgt, der sich am evaluativ-konstruktiven Vorgehen von Achtenhagen et al. (1992) und der partizipativen Konstruktionsidee von Sloane (1992) orientiert sowie auf den neopragmatischen Modellierungsprämissen von Stachowiak (1973), dem epistemologischen Subjektmodell von Groeben und Scheele (1977), dem konstruktivistischen Verständnis von Geschäftsprozessmodellen nach Gaitanides (2012, 99-107) sowie dem kommunikationswissenschaftlichen Begriffsverständnis von Burkart $(2002,61)$ basiert.

5 Den Auswahlkriterien liegt das Komplexitätsverständnis von Dörner (1989) zugrunde. Zudem rekurrieren die Kriterien auf die Forderung von Tramm $(2009,82)$ nach horizontaler und vertikaler Integration kaufmännischer Berufstätigkeit sowie von Reinisch (2012) nach dem Subjektbezug berufs- und wirtschaftspädagogischer Modelle. 
Die Analyse der Prozesse erfolgte dabei aus mehreren Perspektiven: Zum einen wurden quantitative und qualitative Daten zum Verlauf zwischenmenschlicher Kommunikation und Kooperation erhoben und es wurde analysiert, mit welcher Intensität und Wechselseitigkeit Fach- und Führungskräfte Informationen austauschen, welche Medien sie nutzen und wie sie mit Emotionen, insbesondere mit Belastungsempfindungen umgehen. Zum anderen wurde untersucht, welche Ereignisse, Arbeitsaufgaben und Persönlichkeitsmerkmale (Kenntnisse, Fähigkeiten, Fertigkeiten, Einstellungen, Motive) die Kommunikation und Kooperation determinieren bzw. dafür nötig sind.

Zur Datenerhebung und -auswertung wurden unterschiedliche Methoden angewendet: Zum einen wurden Dokumente (Organigramme, Handbücher mit Leitlinien, Prozessbeschreibungen) analysiert. Zum anderen wurden die Mitarbeiterinnen, Mitarbeiter und Führungskräfte online mithilfe eines standardisierten Fragebogens schriftlich befragt $(\mathrm{n}=187)$. Der Fragebogen umfasst 15 geschlossene Fragen mit 153 Items (sowie 870 bedingten Items) und drei offene Fragen mit freien Antwortfeldern. Die Befragten benötigten durchschnittlich eine Stunde Bearbeitungszeit. Zudem wurden leitfadengestützte Intensiv-Interviews ( $\mathrm{n}=20)$ im Umfang von insgesamt 12,5 Stunden (bzw. 270 Transkriptionsseiten) durchgeführt. Die Daten wurden mithilfe netzwerkanalytischer, statistischer und inhaltsanalytischer Verfahren ausgewertet.

\subsection{Einige Befunde zur Kommunikation und Kooperation in Kern- und Unterstützungsprozessen}

Die Daten zeigen, dass die konkrete Kommunikation und Kooperation in den Geschäftsprozessen keinesfalls linear verlaufen, sondern durch zahlreiche Wechselbeziehungen gekennzeichnet und hoch komplex sind. Zudem unterscheiden sich die Kommunikation und Kooperation in den verschiedenen Geschäftsprozessen deutlich voneinander - und zwar sowohl in Inhalt und Form als auch bezogen auf die emotionale Belastung und motivationale Einstellung der Beteiligten (vgl. Schlicht \& Klauser 2014).

Einige Ergebnisse zur Kommunikation und Kooperation wurden unter Nutzung des Methodenrepertoires der Sozialen Netzwerkanalyse (vgl. Jansen 2006; Rehrl \& Gruber 2007) in Form von Netzen abgebildet, um einerseits die Komplexität und um andererseits Unterschiede zwischen den Prozessen sichtbar zu machen. Abbildung 2 (auf der folgenden Seite) zeigt exemplarisch die Kommunikation und Kooperation im Kernprozess „Auftragsbear- 
beitung Biogas“ (linkes Netz) und im Unterstützungsprozess „Kundenzufriedenheitsmanagement" (rechtes Netz). ${ }^{6}$

Abb. 2: Konkrete geschäftsprozessbezogene Kommunikation und Kooperation
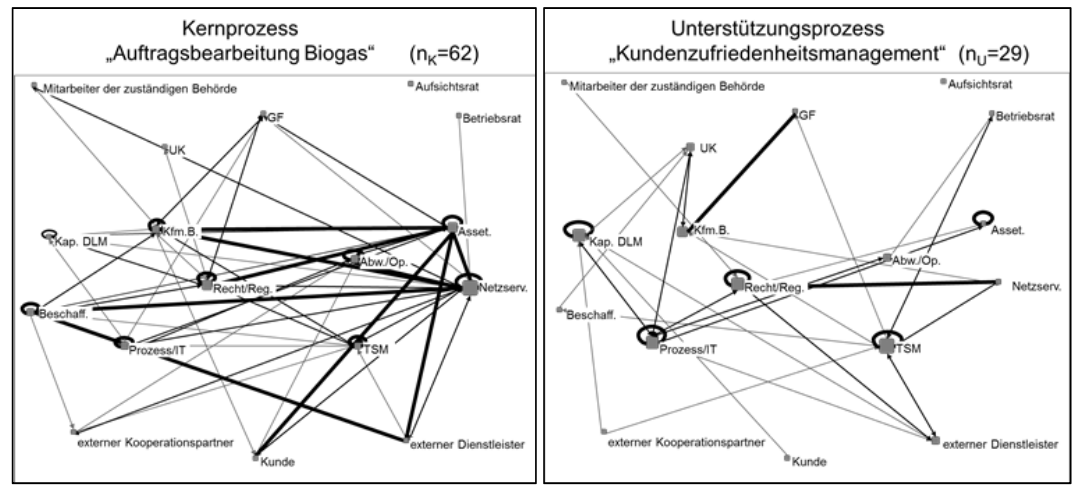

Quelle: Eigene Darstellung.

Zum einen ist dargestellt, welche Mitarbeiter in welchen Organisationseinheiten (Kästchen) tatsächlich miteinander kooperieren. Die Organisationseinheiten des kaufmännischen Bereichs sind jeweils in der linken Hälfte eines Netzes abgebildet, die der technischen Bereiche auf der rechten Hälfte. Die Unternehmensleitung (GF) und Stabsstellen (UK) stehen in der Mitte über den kaufmännischen und technischen Bereichen. Zudem sind jeweils an den Bildrändern Kontrollgremien (Aufsichtsrat, Betriebsrat) und externe Personen eingezeichnet. Je größer ein Kästchen ist, desto mehr Kommunikationsund Kooperationsbeziehungen haben die Mitarbeiter mit anderen Akteuren und desto größer ist ihr Einfluss im Prozess.

Zum anderen wird mithilfe der Linien abgebildet, wie intensiv die Mitarbeiter miteinander kommunizieren und kooperieren. Die Dicke der Linien steht für die Intensität (d. h. die Häufigkeit) des wechselseitig wahrgenommenen Informationsaustauschs. Die relationalen Netzwerkdaten zeigen, dass sowohl in industriellen Kern- als auch in den Unterstützungsprozessen Mitarbeiter kaufmännischer und technischer Fachbereiche intensiv miteinander kommunizieren und kooperieren.

6 Die Netze wurden auf der Grundlage der quantitativen Daten der schriftlichen Befragung mithilfe des Netzwerkanalyse-Programms UCINET erstellt. Die Netzwerkkanten (Linien) basieren auf durchschnittlichen In- und Out-Degrees. Die Position der Netzwerkknoten (Kästchen/Organisationseinheiten) orientiert sich am Organigramm des Unternehmens. Die Knotengröße wird bestimmt durch das relative Zentralitätsmaß. 
Die Netzwerkdaten liefern zudem eine Grundlage für die Optimierung der Geschäftsprozesse im Unternehmen. Die vielen ,dicken“ Linien im Kernprozess waren beispielsweise ein Indiz für Unsicherheit, Intransparenz und für eine „Versuchs- und Irrtums-Kommunikation“ bei der Aufgabenbearbeitung. Das haben vor allem die Interviewdaten deutlich gemacht. Im Unterstützungsprozess sah das anders aus. Die geringe Menge an Kooperationsbeziehungen war bei Nachfrage in den Interviews eher ein Ausdruck für ein mangelndes Verständnis zum Begriff der „Beschwerde“, für Unsicherheit bei der Mediennutzung und für mangelnde soziale Einbindung bzw. die Isolation einzelner Bereiche und Mitarbeiter sowie für damit einhergehende Belastungsempfindungen (vgl. Schlicht \& Klauser 2014).

Auf der Grundlage der Interviewdaten wurden die emotionalen und sozialen Bedingungsfaktoren des Handelns in Geschäftsprozessen einer weitergehenden Analyse unterzogen (vgl. Klauser \& Schlicht 2015). Folgendes Beispiel in Abbildung 3 zeigt, dass das Handeln in Geschäftsprozessen nicht nur von Ereignissen und einer damit verknüpften kognitiv gesteuerten Aufgabenerledigung, sondern auch von Emotionen der beteiligten Personen und vom individuellen Umgang mit Belastungsempfindungen bestimmt wird.

\section{Abb. 3: Emotionen und Umgang mit Belastungsempfindungen (Beispiel)}

„Wenn so ein Projekt kurz vor dem Abschluss steht, dann wird auch jeder so ein bisschen nervös. Funktioniert es? Klappen die Tests? Und gibt das der TÜV frei? Es ist eigentlich ganz normal, dass das dann irgendwie auch mal emotional hochkochen kann, wobei es eigentlich auch nicht der Fall ist, dass einer ins Telefon schreit oder cholerisch wird. Aber der Ton wird dann härter. Das ist so ein typisches Beispiel, wo es schon relativ, eskaliert will ich nicht sagen, aber es war kurz davor. Der Inbetriebnahmetermin wurde zwar verschoben, lag aber noch innerhalb unseres Zeitfensters. Die Anlage tat aber zum Zeitpunkt X nicht das, was sie tun sollte. Sie lief nämlich nicht los. Es waren kapitale Fehler in der Anlage, die beseitigt werden mussten. Bis die Anlage dann los lief, das hat sich über 14 Tage/drei Wochen hingezogen. Die 14 Tage/drei Wochen sind dann natürlich eine haarige Zeit. Da ruft der jeden Tag dreimal an und dann geht es richtig zur Sache. Der macht natürlich Druck, der will einspeisen. Wir können nicht anders. Klar, wenn die Anlage nicht läuft, was wollen Sie dann machen? Dann haben Sie die Leute vor Ort, die alles durchchecken, gucken, wo der Fehler liegt."

\section{Coping-Strategien}

(vgl. Lazarus \& Folkman 1984)

Positiver Vergleich mit anderen Situationen zur Reduzierung emotionaler Anspannung

Selbstverteidigende Umdeutung der Situation zur Reduzierung emotionaler Bedrängnis

Distanzierung zur Belastungsreduzierung

Fehlersuche mithilfe von Kollegen zur Lösung des Problems

Quelle: Eigene Darstellung in Anlehnung an Klauser und Schlicht (2015, 285).

Ein Mitarbeiter beschreibt hier, welche eigenen und fremden Emotionen er bei der Bearbeitung einer Beschwerde wahrgenommen hat. (Die Emotionsbeschreibungen sind kursiv hervorgehoben.) Zudem berichtet er, wie er mit den 
Belastungen umgegangen ist (siehe unterstrichene Aussagen). Dieser Mitarbeiter versucht, das Belastungsempfinden durch Coping-Strategien (vgl. Lazarus \& Folkman 1984) zu reduzieren: Zum einen versucht er, Positives aus der Situation zu ziehen. Zum anderen deutet er die Situation selbstverteidigend um, distanziert sich mithilfe von Verallgemeinerungen und nutzt bei der Fehlersuche und der Lösung des Problems die Unterstützung von Kollegen.

Das ist jedoch bei weitem nicht bei allen interviewten Personen so (vgl. Klauser \& Schlicht 2015). Viele Mitarbeiter arbeiten mit Vermeidungsstrategien, die nur bedingt zur Belastungsreduktion führen bzw. langfristig Gesundheitsschäden zur Folge haben können.

\section{Zusammenfassung und Ausblick}

Aus den Untersuchungsergebnissen lassen sich $u$. a. folgende Konsequenzen für die Lehr-Lern-Prozessgestaltung und Curriculumentwicklung ableiten: Zum einen ist es notwendig, in der Aus- und Weiterbildung die geschäftsprozessbezogene Kommunikation und Kooperation in ihrer Komplexität viel stärker als bisher zum Lerngegenstand zu machen und dabei auch Emotionen und den Umgang mit Belastungsempfindungen in stärkerem Maße als bisher $\mathrm{zu}$ thematisieren sowie in den Kontext von Geschäftsprozessen zu rücken. Coping-Strategien zum effektiven Umgang mit Belastungsempfindungen entwickeln sich nicht en passant im Arbeitsprozess, sondern müssen bereits in der Aus- und Weiterbildung thematisiert und systematisch geübt werden. Das geschieht bisher, wenn überhaupt, eher zufällig und vor allem ohne Bezug zum Handeln in Geschäftsprozessen.

Zum anderen sind neben linearen Abbildungsformen zur Veranschaulichung organisationaler und informationstechnischer Aspekte auch Netzwerkdarstellungen zu nutzen und es sind Fälle zu konstruieren, die das Kommunizieren und Kooperieren von zwei und mehr Personen abbilden, um die Komplexität des Handelns in Geschäftsprozessen sowie Unterschiede zwischen den Prozessen $\mathrm{zu}$ verdeutlichen, um die fachbereichsübergreifende Zusammenarbeit sowie damit verbundene Herausforderungen bei der Verständigung $\mathrm{zu}$ thematisieren und um Ansätze zur Optimierung von Geschäftsprozessen zu diskutieren. Dabei sind auch der Konstruktcharakter sowie die Möglichkeiten und Grenzen von Geschäftsprozessmodellen zu reflektieren. Das betrifft sowohl lineare Abbildungsformen (wie Flussdiagramme und ereignisgesteuerte Prozessketten) als auch netzwerkartige Darstellungen.

In der vorgestellten Untersuchung wurden die geschäftsprozessbezogenen Konzepte und Methoden der Betriebswirtschaftslehre und Wirtschaftsinformatik um die berufs- und wirtschaftspädagogische Perspektive zur Analy- 
se und Modellierung des Handelns in Geschäftsprozessen angereichert. Damit wird es möglich, die Komplexität von Kommunikation und Kooperation in Geschäftsprozessen in unterschiedlichen Unternehmen und Branchen zu untersuchen und die Ergebnisse für die Aus- und Weiterbildung zu nutzen. Das schließt auch die Aus- und Weiterbildung von Handels- bzw. Gewerbelehrern ein.

Es ist davon auszugehen, dass Lehrer derzeit allein mithilfe der fachwissenschaftlichen Ansätze (der BWL und Winf) oder auf der Grundlage eigener betrieblicher Erfahrungen kaum in der Lage sind, z.B. Kaufleute für Büromanagement effektiv auf die Analyse und Optimierung von Geschäftsprozessen vorzubereiten. Um das leisten zu können, müssen sie sich bereits während des Studiums reflektiert mit der Kommunikation und Kooperation in konkreten betrieblichen Geschäftsprozessen auseinandersetzen und entsprechende Fähigkeiten zur curricularen und didaktisch-methodischen Umsetzung entwickeln. Dafür gibt es allerdings bisher kaum empirisch fundierte, hochschuldidaktische Ansätze (vgl. Rebmann \& Schlömer 2009).

Der vorgelegte Ansatz beruht auf der Grundidee, dass sich Mitarbeiterinnen und Mitarbeiter in den Unternehmen an der Analyse und Modellierung von geschäftsprozessbezogener Kommunikation und Kooperation beteiligen. Dafür sind einerseits geeignete Ansätze für Partizipation und Reflexion zu entwickeln und $\mathrm{zu}$ erproben. Andererseits müssen in der beruflichen Ausund Weiterbildung die Fähigkeiten und Fertigkeiten zur partizipativen und reflektierten Geschäftsprozessanalyse und -modellierung systematisch herausgebildet werden. Um das leisten zu können, sind in Anlehnung an Reinisch (2014) mehrere Vermittlungsschritte notwendig. Zum einen ist die betriebliche Realität auf der Grundlage empirischer Befunde für Schulungen und Unterrichte aufzubereiten. Wie die Befunde zeigen, ist dabei u. a. auch die Singularität von Geschäftsprozessen zu thematisieren, die sich aus der Kommunikation und Kooperation von Menschen mit individuell unterschiedlichen Kenntnissen, Fähigkeiten, Fertigkeiten, Einstellungen und Motiven ergibt.

Zum anderen müssen die Ergebnisse für die curriculare Modellierung, einschließlich der Auswahl, Sequenzierung und Komplexitätsgestaltung der Inhalte genutzt werden. In weiterführenden Studien wäre zum Beispiel zu prüfen, inwieweit die in der vorgelegten Untersuchung nach branchenunabhängigen Kriterien ausgewählten Geschäftsprozesse und die partizipativen Modellierungen von Fach- und Führungskräften in schulischen Kontexten genutzt werden können, um das Kommunizieren und Kooperieren in Geschäftsprozessen in ihrer Komplexität, d. h. vernetzt, interdisziplinär, dynamisch sowie für Lernende subjektiv bedeutsam und zudem thematisch verknüpft mit materiellen, finanziellen und informationstechnischen Aspekten beruflicher Tätigkeit zu modellieren. Zudem wäre zu prüfen, inwieweit die vorhandenen fachdidaktischen Modellierungsansätze (z.B. von Achtenhagen 
\& Winther 2011; Getsch \& Preiß 2003; Tramm 2009) um die Perspektive des Kommunizierens und Kooperierens in Geschäftsprozessen erweitert werden können und sollten. Dazu gehört es auch, didaktische Ansätze für die LehrLern-Prozessgestaltung zu entwickeln und zu erproben, die die Lernenden in die Modellierung von Geschäftsprozessen einbeziehen. Wie unsere Untersuchung und die anschließenden Interventionsstudien zeigen, differieren die Bedingungen und (pädagogischen) Herausforderungen dabei in der Ausbildung und in der Weiterbildung beträchtlich (vgl. Schlicht \& Klauser 2014).

\section{Literatur}

Achtenhagen, F. \& Winther, E. (2009). Konstruktvalidität von Simulationsaufgaben: Computergestützte Messung berufsfachlicher Kompetenz am Beispiel der Ausbildung für Industriekaufleute: Abschlussbericht zur Einreichung beim BMBF. Göttingen. Online: https://www.bmbf.de/files/Endbericht_BMBF09.pdf (16.02. 2015).

Achtenhagen, F. \& Winther, E. (2011). Fachdidaktische Perspektiven der Kompetenzmessung - am Beispiel des kaufmännisch-verwaltenden Bereichs. In O. Zlatkin-Troitschanskaia (Hrsg.), Stationen Empirischer Bildungsforschung (S. 352367). Wiesbaden: VS Verlag für Sozialwissenschaften.

Achtenhagen, F., John, E. G., Preiß, P., Tramm, T., Seemann-Weymar, H. \& Schunck, A. (1992). Lernhandeln in komplexen Situationen - Neue Konzepte der betriebswirtschaftlichen Ausbildung. Wiesbaden: Gabler.

Baethge, M. \& Baethge-Kinsky, V. (2004). Der ungleiche Kampf um das lebenslange Lernen. Münster: Waxmann.

Becker, J. \& Kahn, D. (2012). Der Prozess im Fokus. In J. Becker, M. Kugeler \& M. Rosemann (Hrsg.), Prozessmanagement: Ein Leitfaden zur prozessorientierten Organisationsgestaltung, (S. 3-16). Dordrecht: Springer.

Borch, H., Ehrke, M., Müller, K. \& Schwarz, H. (Hrsg.). (1999). Gestaltung der betrieblichen Ausbildung in den neuen IT-Berufen: best practice. Umsetzungsbeispiele aus Klein-, Mittel- und Grossbetrieben. Bielefeld: Bertelsmann.

Burkart, R. (2002). Kommunikationswissenschaft - Grundlagen und Problemfelder. Umrisse einer interdisziplinären Sozialwissenschaft (4. überarb. u. akt. Aufl.). Wien: Böhlau.

Busian, A. (2011). Geschäftsprozessorientierung - curriculare Orientierungsgröße mit Integrationskraft oder Modeerscheinung?bwp@Berufs- und Wirtschaftspädagogik - online, 20, 1-26. Online: http://www.bwpat.de/ausgabe20/busian_bwpat 20. pdf (17.10.2013).

Cersovsky, H., Hunold, F. \& Squarra, D. (2007). Blickpunkt Kunde - Geschäftsprozesse im Einzelhandel: Schülerband (4. Aufl.). Troisdorf: Bildungsverl. EINS.

Dörner, D. (1989). Die Logik des Misslingens. Reinbek: Rowohlt.

Euler, D. \& Hahn, A. (2007). Wirtschaftsdidaktik (2. Aufl.). Bern u. a.: Haupt. 
Fleischmann, A., Schmidt, W., Stary, C., Obermeier, S. \& Börger, E. (2011). Subjektorientiertes Prozessmanagement: Mitarbeiter einbinden, Motivation und Prozessakzeptanz steigern. München: Carl Hanser Verlag.

Gadatsch, A. (2012). Grundkurs Geschäftsprozess-Management. Methoden und Werkzeuge für die IT-Praxis: Eine Einführung für Studenten und Praktiker (7., akt. Aufl.). Wiesbaden: Vieweg+Teubner Verlag.

Gaitanides, M. (2012). Prozessorganisation: Entwicklung, Ansätze und Programme des Managements von Geschäftsprozessen (3. Aufl.). München: Franz Vahlen.

Getsch, U. \& Preiß, P. (2003). Geschäftsprozessorientierter Einsatz integrierter Informationssysteme als Herausforderung für die didaktische Reduktion lernfeldstrukturierter Lehrpläne.bwp@Berufs-und Wirtschaftspädagogik-online, 4, 130. Online: http://www.bwpat.de/ausgabe4/getsch_preiss_bwpat4.pdf (01.06.20 13).

Groeben, N. \& Scheele, B. (1977). Argumente für eine Psychologie des reflexiven Subjekts: Paradigmawechsel vom behavioralen zum epistemologischen Menschenbild (Psychologie und Gesellschaft, Bd. 4). Darmstadt: Steinkopff.

Harms, K. (2014). Ein Plädoyer für das Geschäftsprozessmanagement im neuen Büroberuf. Wirtschaft und Erziehung, 66(4), 186-191.

Jansen, D. (2006). Einführung in die Netzwerkanalyse: Grundlagen, Methoden, Forschungsbeispiele (3., überarb. Aufl.). Wiesbaden: VS Verlag für Sozialwissenschaften.

Klauser, F. \& Schlicht, J. (2015). Emotionen und Belastungserleben im Kontext betrieblichen Handelns - dargestellt am Beispiel der Beschwerdebearbeitung in einem Unternehmen der Energiewirtschaft. In A. Rausch, J. Warwas, J. Seifried \& E. Wuttke (Hrsg.), Konzepte und Ergebnisse ausgewählter Forschungsfelder der beruflichen Bildung: Festschrift für Detlef Sembill, (S. 271-290). Baltmannsweiler: Schneider Verlag Hohengehren.

Lazarus, R. S. \& Folkman, S. (1984). Stress, Appraisal, and Coping. New York: Springer.

Nickolaus, R. (2015). Kompetenzmessung und Kompetenzmodellierung in der beruflichen Bildung. Zeitschrift für Berufs- und Wirtschaftspädagogik, 111(2), 161167.

Rebmann, K. \& Schlömer, T. (2009). Lernen im Prozess der Arbeit. bwp@Berufsund Wirtschaftspädagogik - online, Profil 2, 1-17. Online: http:/www.bwpat.de/ profil2/rebmann_schloemer_profil2.pdf (25.10.2013).

Rehrl, M. \& Gruber, H. (2007). Netzwerkanalysen in der Pädagogik: Ein Überblick über Methode und Anwendung. Zeitschrift für Pädagogik, 52(2), 243-264.

Reinisch, H. (14.11.2012). Thesen zum Workshop „Konzeptionen des kaufmännischen Denkens und Handelns für Berufskonstruktionen“ (BIBB-Fachtagung „Kaufmännische Berufe zwischen Theorie und Ordnungspraxis"). Bundesinstitut für Berufsbildung: Bonn. Online: http://www.bibb.de/dokumente/pdf /Foliensatz_ WS3_Reinisch_final.pdf(20.11.2015).

Reinisch, H. (2014). Sequenzierung und Reduktion - Notizen zu gegenwärtig in der Didaktik des wirtschaftsberuflichen Unterrichts vernachlässigten Aspekten didaktischer Konstruktion vor dem Hintergrund der curricularen Leitlinie „Arbeitsund Geschäftsprozesse“.bwp@Berufs-und Wirtschaftspädagogik-online, Profil 3, 1-17. Online: http://www.bwpat.de/profil3/reinisch_profil3.pdf (23.05. 2014). 
Scheer, A.-W. \& Thomas, O. (2009). Verfahren und Werkzeuge der Informationsmodellierung. In H.-J. Bullinger, D. Spath, H.-J. Warnecke \& E. Westkämper (Hrsg.), Handbuch Unternehmensorganisation: Strategien, Planung, Umsetzung, (3., neu bearb. Aufl, S. 544-568). Berlin: Springer.

Schlicht, J. \& Klauser, F. (2014). Analyse, Konstruktion und Optimierung von Geschäftsprozessen in Unternehmen der Energiewirtschaft (Unveröffentlichter Arbeitsbericht). Institut für Wirtschaftspädagogik der Universität Leipzig.

Schmelzer, H. J. \& Sesselmann, W. (2013). Geschäftsprozessmanagement in der Praxis: Kunden zufrieden stellen - Produktivität steigern - Wert erhöhen (8., überarb. u. erw. Aufl.). München: Carl Hanser.

Schmidt, W., Fleischmann, A. \& Gilbert, O. T. (2009). Subjektorientiertes Geschäftsprozessmanagement. HMD Praxis der Wirtschaftsinformatik, 46(2), 52-62. doi:10.1007/BF03340343.

Sloane, P. F. E. (1992). Modellversuchsforschung: Überlegungen zu einem wirtschaftspädagogischen Forschungsansatz. Wirtschafts-, Berufs- und Sozialpädagogische Texte: Vol. 18. Köln: Müller Botermann.

Spath, D. (2009). Grundlagen der Organisationsgestaltung. In H.-J. Bullinger, D. Spath, H.-J. Warnecke \& E. Westkämper (Hrsg.), Handbuch Unternehmensorganisation: Strategien, Planung, Umsetzung, (3., neu bearb. Aufl.), (S. 3-24). Berlin: Springer.

Stachowiak, H. (1973). Allgemeine Modelltheorie. Wien: Springer.

Staud, J. (2006). Geschäftsprozessanalyse: Ereignisgesteuerte Prozessketten und objektorientierte Geschäftsprozessmodellierung für Betriebswirtschaftiche Standardsoftware (3. Aufl.). Berlin: Springer.

Stauss, B. \& Seidel, W. (2014). Beschwerdemanagement: Unzufriedene Kunden als profitable Zielgruppe (5., vollst. überarb. Auflage). München: Carl Hanser.

Tramm, T. (2004). Geschäftsprozesse und fachliche Systematik - zur inhaltlichen Einführung.bwp@Spezial 1, 134-139. Online: http://www.bwpat.de/spezial1/ ws3_bwpat_spezial1.pdf(10.09.2014)

Tramm, T. (2009). Von der Geschäftsprozess- zur Lernprozessperspektive. Das Zusammenspiel von Prozessorientierung, systemischer Perspektive und prozessübergreifender Kompetenzentwicklung im lernfeldstrukturierten Berufsschulunterricht. In H. Pongratz, T. Tramm \& K. Wilbers (Hrsg.), Prozessorientierte Wirtschaftsdidaktik und Einsatz von ERP-Systemen im kaufmännischen Unterricht, (S. 77-101). Aachen: Shaker.

Tramm, T. \& Reetz, L. (2010). Berufliche Curriculumentwicklung zwischen Persönlichkeits-, Situations- und Wissenschaftsbezug. In R. Nickolaus, G. Pätzold, H. Reinisch, \& T. Tramm (Hrsg.), Handbuch Berufs- und Wirtschaftspädagogik, (S. 220-226). Bad Heilbrunn: Klinkhardt.

Tramm, T., Adler, J.-H., Frost, G., Goldbach, A., Seidler, D. \& Wichmann, E. (2007). Prozessorientierte Wirtschaftslehre (3. Aufl.). Troisdorf: Bildungsverl. EINS.

Weber, J. E., Schmidt, W. \& Weber, P. (2012). Using Social Network Analysis and Derivatives to Develop the S-BPM Approach and Community of Practice. In C. Stary (Hrsg.), S-BPM ONE - Scientific Research: 4th International Conference, S-BPM ONE 2012, Vienna, Austria, April 4 - 5, 2012; Proceedings, (S. 205-217). Heidelberg: Springer.

Wilbers, K. (2012). Wirtschaftsunterricht gestalten: Eine traditionelle und handlungsorientierte Didaktik für kaufmännische Bildungsgänge. Berlin: epubli. 
Wuttke, E. (2005). Unterrichtskommunikation und Wissenserwerb: Zum Einfluss von Kommunikation auf den Prozess der Wissensgenerierung. Frankfurt am Main: Peter Lang.

Wuttke, E., Seifried, J., Brandt, S., Rausch, A., Sembill, D., Martens, T. \& Wolf, K. D. (2015). Modellierung und Messung domänenspezifischer Problemlösekompetenz bei angehenden Industriekaufleuten. Zeitschrift für Berufs- und Wirtschaftspädagogik, 111(2), 189-207. 


\title{
Nutzung von digitalen Medien an berufsbildenden Schulen - Notwendigkeit, Rahmenbedingungen, Akzeptanz und Wirkungen
}

\author{
Alexandra Bach (geb. Eder)
}

\section{Problemstellung}

Die Integration von digitalen Medien in die allgemeine und berufliche Bildung und der damit verbundene Medienkompetenzerwerb werden spätestens seit der Klassifikation unserer Gesellschaft als Informationsgesellschaft gegen Ende der 1990er Jahre als kulturell und wirtschaftlich grundlegend eingestuft (vgl. Bos et al. 2014, 7; Eder 2009, 17; Jenewein 2014, 47; Pferdt \& Kremer 2012, 290). Demgemäß deklariert die Europäische Kommission die „digitale Medienkompetenz“ als eine der acht elementaren Schlüsselkompetenzen bzw. als eine zentrale Kulturtechnik, die das lebenslange Lernen, die Beschäftigungsfähigkeit sowie die gleichberechtigte Partizipation der Einzelnen in der Informations- und Wissensgesellschaft ermöglicht (vgl. Europäische Kommission 2010, 29; Senkbeil et al. 2014, 83). Es gilt folglich, eine zeitgemäße digitale Medienbildung bzw. Medienkompetenzvermittlung im (Berufs-)Bildungssystem zu realisieren. Weiterhin benötigt unsere wissensbasierte Gesellschaft Fachkräfte mit ausreichenden digitalen Kompetenzen in allen Wirtschaftsbereichen, um die internationale Wettbewerbsfähigkeit Deutschlands im „digitalen Binnenmarkt für Europa“ zu erhalten und zu stärken und einem potenziellen Fachkräftemangel entgegenzuwirken (vgl. BIBB 2013, 383).

Die Nutzung der digitalen Medien und die digitale Medienbildung an den deutschen Schulen werden jedoch in den unterschiedlichen internationalen OECD-Vergleichsstudien der vergangenen Jahre (aktuell z.B. ICILS 2013: International Computer and Information Literacy Study) als deutlich unter den Anforderungen liegend beurteilt (vgl. Bos et al. 2014, 20; Conrad \& Schuhmann 2015, 131), d.h. Deutschland liegt - die Computernutzung im Unterricht betreffend - in der Regel an letzter Stelle im Vergleichsranking der an der Studie beteiligten OECD-Staaten. Die Situation an den berufsbildenden Schulen kann hier hingegen kaum beurteilt werden, da dazu nur Fragmente an empirischen Daten bzw. keine aktuellen Studien vorliegen (vgl. Eder 2015, 16ff.; Wilbers 2012, 38). Wilbers kommt jedenfalls für die berufliche Bildung zu dem Ergebnis, dass digitales Lehren und Lernen ,in den berufsbildenden Schulen bislang nur eine geringe Rolle [spielen].“(Wilbers 2012, 38). Erst seit etwa zehn Jahren lässt sich verstärkt feststellen, dass in der beruflichen Bildung die Integration digitaler Medien, z.B. durch mehrere Förderprogramme des BMBF zum Thema „Digitale Medien in der Beruflichen Bildung" mit mehr Nachdruck als bisher gefördert wird (vgl. Neumann \& Ueberschaer 2014, 219). Dies erscheint auch notwendig, denn vor dem Hintergrund der 
zentralen Bedeutungszuschreibung, die einer adäquaten Medienbildung der Bevölkerung und der schulischen und außerschulischen Mediennutzung zugeschrieben wird, erscheint eine im internationalen Vergleich weit unterdurchschnittliche Mediennutzung im Bildungssystem als wenig akzeptabel. Vor allem erscheint es auch notwendig, hier regelmäßige statistische Untersuchungen, die berufliche Bildung betreffend, durchzuführen, um überhaupt empirisch fundierte Aussagen treffen und Qualitätsentwicklungsmaßnahmen einleiten zu können. Diesen Sachstand nimmt dieser Beitrag zum Ausgangspunkt. Es stellt sich die Frage, welche Faktoren die Nutzung digitaler Medien durch das Berufsbildungspersonal (hier mit dem Fokus auf die Lehrkräfte) begünstigen bzw. behindern. In engem Zusammenhang damit steht auch die Frage, welcher Mehrwert bzw. welcher Nutzen mit einer digitalen Medienverwendung intendiert ist, deshalb wird in Kapitel 2 zunächst skizziert, welche grundlegenden Argumentationslinien diskutiert werden, die die Notwendigkeit einer Implementierung digitaler Medien herausstellen. Danach werden in Kapitel 3 im Überblick repräsentative empirische Ergebnisse zur Medienintegration an deutschen Schulen vorgestellt, um nachfolgend - in Kapitel 4 nach empirisch fundierten Gelingensbedingungen der Mediennutzung zu recherchieren. Dies erfolgt mit Rückgriff auf die Technologie-Akzeptanzforschung, welche sich seit den 1980er Jahren etablierte und deren Modelle bis heute immer weiter ausdifferenziert werden. Das abschließende Fazit und die Handlungsempfehlungen folgen in Kapitel 5.

\section{Erwartungshaltung und Notwendigkeit der Integration digitaler Medien in der beruflichen Bildung}

Wenn es um die Implementierung digitaler Medien in das berufliche oder allgemeine Bildungssystem geht, werden in der Regel drei bildungspolitisch bzw. pädagogischdidaktisch motivierte Begründungslinien benannt. Diese werden im Folgenden vorgestellt, da es für potenzielle Anwender einer Technologie ein zentraler Motivationsfaktor ist, welcher Nutzen mit der Anwendung einer Technologie verbunden ist (vgl. Venkatesh, Thong \& Xu 2012, 159).

Begründungslinie (1): Die digitale „Medienkompetenz wird (.) als eine der wesentlichen Voraussetzungen für eine aktive, umfassende bürgerschaftliche Teilhabe und für die Vermeidung bzw. Verringerung der Gefahr eines Ausschlusses aus dem gesellschaftlichen Leben gesehen." (Europäische Kommission 2010, 10). Sie wird als zentrale Schlüsselkompetenz und Kulturtechnik klassifiziert, die bei deren Fehlen zur Benachteiligung in der Gesellschaft führt (vgl. Egloffstein et al. 2012, 223; Eickelmann, Gerick \& Bos 2014, 26). Dies liegt in der Omnipräsenz digitaler Medien begründet, die mittlerweile in allen privaten, öffentlichen und beruflichen Bereichen etabliert sind und vor allem auch selbstverständlicher Bestandteil der Lebenswelt der Auszubildenden/ Schüler(-innen) sind (vgl. Pferdt \& Kremer 2012, 290; Senkbeil et al. 2014, 83; Wilbers 2012, 39). Digitale Medienkompetenz „,ist eine Querschnittskompetenz in praktisch allen Berufen und muss entsprechend vermittelt bzw. in die Aus- und Weiterbildung 
integriert werden.“ (Weiß 2012, 3). Doch gilt es, den Begriff der digitalen Medienkompetenz konkret zu fassen. Ursprünglich wurde der Begriff Medienkompetenz in den 1970er Jahren von Dieter Baacke etabliert, der den sozialwissenschaftlichen Begriff der „kommunikativen Kompetenz" von Habermas in den medienpädagogischen Kontext übertrug (vgl. Baacke 2004, 23). Kommunikative Kompetenz ist in diesem Sinne die Fähigkeit des Einzelnen, sich direkt durch Sprache oder indirekt durch Medien zu verständigen, und zwar mit dem Ziel, das Zusammenleben in der Gesellschaft mitzugestalten und gleichberechtigt daran teilzunehmen. Medienkompetenz ist folglich die Fähigkeit, vermittelt durch Medien (hier: digitaler Medien) kommunikativ zu handeln (vgl. Schorb 2005, 258). Dazu gehören auch psychische Fähigkeiten, die verhindern, dass die Nutzerinnen in der Medienvielfalt die Selbstkontrolle verlieren (vgl. Schulmeister 2012, 43). Zusammengefasst umfasst (digitale) Medienkompetenz - wie sie hier in diesem Beitrag interpretiert wird - die Dimensionen a) das Medienwissen, d.h. das Wissen über Funktionsweise und Struktur von (digitalen) Medien(systemen), und b) das Medienhandeln, d.h. die Fähigkeit, (digitale) Medien kompetent zu nutzen, diese zu gestalten und über die medienvermittelte Kommunikation an gesellschaftlichen Entscheidungsprozessen zu partizipieren, und c) die Medienbewertung zielt hingegen darauf ab, Bürger(innen) zu befähigen, Problemstellungen, wie Daten- und Verbraucherschutz, Schutz der Privatsphäre, Werte und Normen, Barrierefreiheit in der digitalen Welt, kontrovers zu diskutieren und zu reflektieren (vgl. BIBB 2013, 404; Eder 2009, 20; Egloffstein et al. 2012, 223). Besonders bedeutsam ist in diesem Kontext die Fähigkeit, „Inhalte und Kommunikationsprozesse in den Medien zu verstehen und kreativ an ihnen mitzuwirken“ (Schulmeister 2012, 44). So wird es Individuen durch den Zugang und den kompetenten Umgang mit digitalen Medien ermöglicht, an der öffentlichen Meinungsbildung und an den demokratischen Entscheidungs- und öffentlichen Verwaltungsprozessen zu partizipieren. Wer an diesen Digitalisierungsprozessen nicht partizipieren kann, wird mit größerer Wahrscheinlichkeit ausgeschlossen, benachteiligt und in seinen gesellschaftlichen Handlungsmöglichkeiten beschnitten (vgl. Senkbeil et al. 2014, 84).

Von der Teilhabe an der digitalen Welt sind bestimmte Personengruppen in der Europäischen Gemeinschaft aktuell in besonders starkem Maße ausgeschlossen. 150 Mio. Europäer, d.h. knapp ein Drittel, haben noch nie das Internet genutzt (vgl. Europäische Kommission 2010, 29). Vor allem Personen mit höherem Alter, Behinderung, geringem Einkommen und Bildungsstand sowie Arbeitslose sind von dieser oder ähnlich ausgeprägter digitaler Spaltung betroffen, häufig auch aufgrund von mangelnden Medienkompetenzen. Auch die Jugendlichen in Deutschland, die in der Regel Zugang zu digitalen Medien haben, sind nur teilweise dazu in der Lage, sich aufgabenorientierte digitale Medienkompetenzen autodidaktisch anzueignen. Die aktuelle Studie des Medienpädagogischen Forschungsverbunds Südwest (MPFS) von 2015 zeigt (wie auch in den Jahren davor), dass Heranwachsende im Alter von 12-19 Jahren $(n=1200)$ sehr gute Zugangsmöglichkeiten zu Computer und Internet haben, d.h. sie leben in 99 Prozent der Fälle in Haushalten, in denen ein ans Internet angeschlossener Computer/Laptop zur Verfügung steht (vgl. MPFS 2015, 6), oder verfügen sogar über ein eigenes Gerät (75 Prozent). Dennoch ist dies kein Indikator dafür, dass die 12-19-Jährigen eine aufgaben- 
orientierte Nutzung praktizieren. Etwa 51 Minuten werden im Durchschnitt pro Werktag darauf verwandt, am Computer Schulaufgaben zu erledigen (vgl. ebd., 30). Befragt man die Jugendlichen zum zeitlichen Nutzungsumfang der Onlineaktivitäten - die insgesamt von 94 Prozent der Befragten täglich bis mehrmals pro Woche getätigt werden (vgl. ebd., 11) -, so kann jedoch festgestellt werden, dass hier die Kommunikation von 44 Prozent als die bedeutendste Kategorie eingestuft wird, gefolgt von Unterhaltungsaktivitäten, die Musik, Bilder oder Videos betreffen (25\%), Online-Spielen (18\%) und Informationssuche $13 \%$ (vgl. ebd., 26). Auch in der aktuellen ICILS-Studie ${ }^{1}$ (International kommt man zusammenfassend zum Ergebnis: „Many 'digital natives' are not digitally competent - school has a key role to play. Being born in a digital era is not a sufficient condition for being able to use technologies in a critical, creative and informative way.“ (Europäische Kommission 2014, 5). In jedem der 21 Länder, die an der ICILSStudie teilnahmen, weisen mindestens 25 Prozent der teilnehmenden Schüler(-innen) sehr niedrige computer- und informationsbezogene Kompetenzen auf. Die Gefahr einer digitalen Spaltung ist hier spürbar (vgl. Senkbeil et al. 2014, 85). Die Schulen müssen hier unterstützend tätig werden und die Jugendlichen dazu motivieren, arbeitsmarktund lebensrelevante digitale Medienkompetenzen aufzubauen. Korrespondierend damit postuliert auch das BIBB: "Qualifizierte Ausbilder und Ausbilderinnen in den Betrieben und qualifizierte Lehrerinnen und Lehrer an den Berufsschulen sind diejenigen, die Auszubildende für einen reflektierten Umgang mit digitalen Medien sensibilisieren müssen." (BIBB 2013, 402).

Eine weitere Begründungslinie (2) zur Integration digitaler Medien ins (Berufs-) Bildungssystem besteht darin, die didaktisch-methodischen Potenziale digitaler Medien für institutionalisierte und individuelle Lehr-Lernprozesse zu nutzen. Damit ist die Annahme verbunden, dass individuelles, kooperatives, selbstgesteuertes, zeit- und ortsunabhängiges, differenziertes und auch lebenslanges Lernen besser als mit analogen Medien realisiert werden kann (vgl. Egloffstein, Kögler \& Kärner 2012, 223; Jenewein 2014, 48; Pferdt \& Kremer 2012, 290). Zudem ,lassen sich [z.B. auf Basis empirischer Schulleistungsstudien, wie z.B. PISA] für einen ziel- und problemorientierten Umgang mit Informations- und Kommunikationstechnologien förderliche Effekte auf bereichsspezifische Kompetenzen (z.B. Mathematik, Naturwissenschaften, Fremdsprachen) ermitteln." (vgl. Senkbeil et al. 2014, 84), die jedoch nicht als gesichert gelten, da die Messung der Computerkompetenz häufig auf Selbsteinschätzung beruht. Weitere Wirkungsvermutungen lauten, dass der sachgerechte Einsatz digitaler Medien zu einer erhöhten Lernleistung, Motivation, Handlungsspielräumen und Verfügbarkeit von Inhalten führt bzw. die Unterrichtsqualität damit gefördert wird (vgl. Egloffstein et al. 2012, 223). Für die berufliche Bildung verspricht man sich vor allem durch die digitalen Medien auch eine verbesserte Förderung der umfassenden beruflichen Handlungskompetenz (vgl. BIBB 2013, 408; Jenewein 2014, 53). Zu dieser Annahme führt unter anderem auch die stetig wachsende Vielfalt an digitaler Software, Hardware und LehrLernvarianten, die für berufliche, schulische und individuelle Lernprozesse genutzt

1 Befragt wurden Achtklässler (Deutschland: $n=2.225$ ) in jeweils ca. 150 Schulen pro Land von 21 Ländern der OECD (vgl. Bos et al. 2014, 12f.) 
werden können. Es wird von einer ,explosion of IT tools“ gesprochen (vgl. Bos et al. 2014, 116). So stehen auf Softwareebene z.B. Web \& Computer Based Trainings (CBT, WBT), Learning Management Systeme, Blogs, Wikis, Podcast, Augmented Reality, Simulationen, Serious Games und Soziale Netzwerke etc. zur Verfügung (vgl. BIBB 2013, 394), auf der Technikebene können z.B. Computer, Tablets, digitales Whiteboards, Smartphones, technische Lernsysteme oder reale Maschinen für die Realisierung digitaler Lehr-Lernvarianten, wie z.B. E-Learning, mobile \& blended Learning, digital gestützte Präsenzseminare usw. herangezogen werden (vgl. Howe 2013, 3f.; Pferdt \& Kremer 2012, 289).

So wird durch die verfügbaren Bildungstechnologien ein weiter „didaktischer Raum" des formellen und informellen Lernens eröffnet, der sich durch die Dimensionen Medienfunktion (Medien als Träger von Information, Kommunikation, Handlungen), Sozialform (Einzelarbeit, Gruppenarbeit, Plenum) und Lernaktivität (z.B. Informieren, Diskutieren, Kooperieren, Problemlösen, Produzieren) on- oder offline beschreiben lässt (vgl. Eder 2009, 45; Pferdt \& Kremer 2012, 297ff.). Symptomatisch für die Integration digitaler Medien in die berufliche Bildung ist dabei ebenfalls die ,starke Verschmelzung des methodischen und des inhaltlichen Aspekts insbesondere dort, wo digitale Medien als Teil der Arbeitswelt (...) in beruflichen Schulen aufgegriffen werden (...). So stellt [beispielsweise] die (.) Programmierung speicherprogrammierbarer Steuerungen (SPS) in didaktischer Hinsicht eine nicht auflösbare Verschmelzung eines Lernens für Medien und mit Medien dar. Dabei lassen sich Anwender/-innen digitaler Medien in beruflichen Handlungsfeldern (...) von Expertinnen und Experten für digitale Medien unterscheiden, beispielsweise (...) Fachinformatiker/-innen.“ (Wilbers 2012, 39).

In der beruflichen Bildung wird aktuell vor allem jedoch das arbeitsplatznahe mobile Lernen als kompetenzförderlich diskutiert. Die zunehmende Digitalisierung der Arbeitswelt fordert ,, von der Berufsbildung Konzepte für die aktive Gestaltung beruflichen Lehrens und Lernens in der Aus- und Weiterbildung." (BIBB 2013, 383). Facharbeiter sollen durch mobile Assistenzsysteme, wie z.B. Tabletcomputer, Datenbrillen - die Augmented Reality ermöglichen - in ihrem Kompetenzentwicklungs- und Problemlöseprozess arbeitsnah und anlassbezogen unterstützt werden (vgl. Eder 2015, 24f.). Darüber hinaus fehlt ,schlichtweg die Zeit, um jedes Mal einen Kurs zu besuchen oder ein Selbstlernprogramm zu bearbeiten. Das Lernen erfolgt überwiegend selbst organisiert im Prozess der Anwendung. Es wird unterstützt durch den Austausch mit Peers oder Expertinnen und Experten.“(Weiß 2012, 3). Damit wird die Verzahnung der Lern- und Arbeitsprozesse angestrebt, indem Zusatzinformationen, wie Videos, Pläne, Informationstexte (abgelegt an Maschinen oder Geräten, abrufbar z.B. durch QR-Code o.Ä.), bedarfsgerecht abgerufen und Problemlösungen ggf. in virtuellen Expertengemeinschaft diskutiert werden können (vgl. Grantz, Karges \& Richter 2014; de Witt 2013, 20ff.). Durch diese mobilen, arbeitsplatznahen Lernformen sollen sich die Arbeitnehmerinnen und Arbeitnehmer für den zukünftigen technologischen Wandel in der Arbeitswelt - der sich aktuell durch Zukunftsprojekte, wie Smart Grid ${ }^{2}$, Smart Factory ${ }^{3}$ usw., und durch

2 Intelligente Energiesysteme

3 Intelligente Fabriken 
eine immer kürzer werdende Halbwertzeit des Wissens kennzeichnen lässt - möglichst selbstgesteuert und bedarfsgerecht wappnen (vgl. BIBB 2013, 391). Ist Lernen im Prozess der Arbeit nicht möglich, gelinge es, zumindest durch digitale Medien - so die Argumentation von Howe: ,in besonderer Weise die Arbeitsprozessorientierung als curriculare und didaktische Leitidee der Berufsbildung [zu] befördern (.), [indem] digitale Medien in Form von Fotos, Videos, Animationen und Simulationen, (...) Arbeitsprozesse als „Lernanker“ relativ authentisch, vielfältig eingebettet, komplex und ganzheitlich (.) präsentieren.“ (Howe 2013, 1f.).

$\mathrm{Zu}$ diskutieren bleibt jedoch, inwieweit in solchen Lehr-Lernsettings ein ganzheitlicher emanzipatorischer Bildungsanspruch realisiert werden kann und sich mobiles Lernen tatsächlich als besonders erfolgreiches Lernen erweist. Denn schon seit mehreren Jahrzehnten zeigt sich dann eine ambivalente Befundlage, wenn es um die Frage geht, ob die Nutzung digitaler Medien zu Lehr-Lernzwecken zu einer erhöhten Lernleistung führt. So weist die Hattie-Studie beispielsweise relativ hohe Effektstärken zum Themenfeld „Computergestützter Unterricht“" $(d=0,37)$ auf (vgl. Hattie, Beywl \& Zierer 2013, 261), während die Effektstärken zum Themenfeld „Webbasiertes Lernen“ (d=0,18) deutlich geringer ausfallen (vgl. Hattie et al. 2013, 268). Konsens ist, dass nicht die Nutzung oder die Verfügbarkeit des Mediums per se zu einer erhöhten Lernleistung führt, sondern dass eine den Lernzielen und der Zielgruppe angemessenes didaktisches Lehr-Lernszenario den Medieneinsatz flankieren muss (vgl. Herzig 2014, 22; Howe 2012, 12), da nur die Kombination der unterschiedlichen Faktoren (z.B. LehrLernstrategien, Vorübung, Feedback etc.) eine optimale Lernleistung ermöglicht (vgl. Conrad \& Schumann 2015, 131; Eder 2015, 38). So wird zum Beispiel in der HattieStudie festgestellt, dass der digitale Medieneinsatz im Hinblick auf problemlösendes Lernen in den analysierten Metastudien mit hoher Wirkung realisiert wurde $\left(\mathrm{d}=0,57^{4}\right)$ (vgl. Hattie et al. 2013, 262). Weiterhin wurde konstatiert, dass digitale Medien dann eine stärkere Wirkung entfalten, wenn sie die Lehr-/Coachingtätigkeit des Bildungspersonals ergänzen $(d=0,45)$ und nicht als Ersatz dafür dienen $(d=0,30)($ vgl. Hattie et al. 2013, 268). Ebenso wenig scheint der Einsatz der digitalen Medien per se als Katalysator für eine konstruktivistische, Lerner zentrierte Lehr-Lernkultur zu wirken - wie häufig vermutet wird (vgl. Egloffstein et al. 2012, 224) - denn empirische Studien deuten darauf hin, dass das Bildungspersonal nicht aufgrund eines Medienwechsels automatisch sein Unterrichtskonzept verändert, sondern dazu tendiert, seine Lehrgewohnheiten mit digitalen Medien weiterzuführen (vgl. Eder 2009, 64f.). Ebenso müssen die Lerner über die Kompetenz verfügen, ihren Lernprozess weitgehend selbstständig zu steuern und zu regulieren. Inwieweit arbeitsplatznahes und bedarfsorientiertes Lernen, z.B. mit Augmented Reality, folglich als besonders wirksam einzustufen ist bzw. unter welchen Bedingungen dies erfolgen muss, gilt es, noch empirisch zu überprüfen, und kann nicht pauschal vorausgesetzt werden (vgl. Conrad \& Schumann 2015, 131).

Weiterhin lautet eine sehr stichhaltige Begründungslinie (3), dass in vielen Lernfeldern der ca. 345 anerkannten Ausbildungsberufe der kompetente Umgang mit digitalen

4 Die Einschätzung basiert auf vier Metastudien, 68 Effekte wurden berücksichtigt. 
Medien (z.B. Software, Hardware, Programmierung usw.) zentrales Unterrichtsziel und damit obligatorisch ist (vgl. Eder 2015, 23; Grantz et al. 2014, 54; Wilbers 2014, 39). Dies ist naheliegend, da in nahezu allen Wirtschaftszweigen Deutschlands digitale Medien zentrale Arbeitsmittel zur Umsetzung von Arbeits- und Geschäftsprozessen sind. Vor allem in den gewerblich-technischen Berufen der Metall- und Elektroindustrie steht mit der Entwicklung hin zu Industrie 4.0 ein neuer Quantensprung der Digitalisierung der Facharbeit bevor (vgl. Schütte \& Mansfeld 2013, 304). „Wartungs- und Instandhaltungsarbeiten, z.B. in der Automobilwirtschaft, sind [auch heute] nur noch mittels computergestützter Diagnosesysteme möglich. In der Metalltechnik repräsentieren die ITinduzierten Unterstützungssysteme für die CNC- und HSC-Technik (computerized Numerical Control und High Speed Cutting) moderne Arbeitsumgebungen" (BIBB 2013, 386) für Facharbeiter auf mittlerem Qualifikationsniveau. Eine repräsentative Befragung des BIBB von 20.000 Erwerbstätigen in Deutschland im Jahr 2012 ergab, dass 71 Prozent der Befragten Computertechnologien als IT-Anwender nutzen und 10 Prozent eine noch weiterführende Nutzung als IT-Experten praktizieren. Im Durchschnitt werden - so das BIBB - in 48 Prozent der Arbeitszeit Computertechnologien verwendet. Dieser technologische Wandel führt dazu, dass vor allem in industriellen Ausbildungsberufen (vgl. BIBB 2013, 384), aber auch im öffentlichen Dienst, im Handwerk und im Handel „, (...) die professionelle Handhabung dieser Technologien in Abhängigkeit vom Ausbildungsberuf - in hohem Maße integraler Bestandteil der beruflichen Handlungskompetenz und zentrales Kompetenzziel in den entsprechenden Lernfeldern“" (Eder 2015, 24) ist.

\section{Nutzung digitaler Medien an allgemeinen und beruflichen Schulen}

Vor dem Hintergrund der Begründungslinien in Abschnitt 2 erscheint es unstrittig, dass eine angemessene Integration digitaler Medien in berufliche und allgemeinbildende Schulen unerlässlich ist (vgl. Eickelmann, Gerick \& Bos 2014, 28ff.). Aktuell werden jedoch die Akzeptanz digitaler Medien an deutschen Schulen und damit auch die zeitgemäße Förderung digitaler Medienkompetenzen als defizitär eingestuft. Definiert wird dabei die Akzeptanz als positive Annahmeentscheidung einer Technologie durch den Nutzer (vgl. Nistor, Wagner \& Heymann 2012, 345). Im schulischen Kontext bedeutet dies vor allem auch die Akzeptanz digitaler Medien im schulischen Unterricht. Unterschieden werden dabei zum einen die Einstellungsakzeptanz, d.h. im Bezugsfeld Unterricht die Absicht einer Person, die digitalen Medien im Unterricht aufgabenbezogen zu nutzen, und zum anderen die Nutzungsakzeptanz, d.h. die real ausgeführte Nutzung im Unterricht. Folgend wird nun die Akzeptanz bzw. Nutzung digitaler Medien an den berufs- und allgemeinbildenden Schulen dargestellt. Die Daten zu den allgemeinbildenden Schulen werden deshalb berücksichtigt, da zur Mediennutzung an den berufsbildenden Schulen keine repräsentativen Daten vorliegen (vgl. Eder 2015, 27f.; Wilbers 2014, 38). 
Seit mehreren Jahren rangiert Deutschland im OECD-Vergleich (ICILS 2013) immer $^{5}$ dann auf dem letzten Rang, wenn es um die Nutzung digitaler Medien im schulischen Unterricht geht. Etwa 1/3 der in der ICILS-Studie befragten deutschen Lehrkräfte nutzt Computer im Jahr 2013 mindestens einmal wöchentlich im Unterricht der achten Jahrgangsstufen und lediglich 9 Prozent nutzen diese täglich (vgl. Bos et al. 2014, 20). In sehr medienaffinen Ländern, wie z.B. Kanada und Australien, nutzen 3/4 bzw. 2/3 der befragten Lehrkräfte digitale Medien täglich im Unterricht (vgl. Eickelmann et al. 2014, 204). Hinzu kommt, dass der Medieneinsatz an deutschen Schulen wenig lernförderlich und eher einfallslos umgesetzt wird - d.h. es werden überwiegend Internetrecherchen und Präsentationsprogramme genutzt anstelle von z.B. anspruchsvollen Simulationsprogrammen. Darüber hinaus korreliert im Gegensatz zu anderen OECD-Ländern die Nutzung digitaler Medien im Unterricht nicht mit der Medienkompetenz der Schüler(innen) und generell wird die Medienkompetenzentwicklung nicht in besonderem Maße als Bildungsziel angestrebt (vgl. Eickelmann 2014, 224). „Weit unterdurchschnittlich““ lautet abschließend das Fazit zur schulischen Nutzung der digitalen Medien in Deutschland (vgl. Bos et al. 2014, 20; Egloffstein et al. 2012, 224). Fragezeichen wirft zudem die aktuelle Situation an den berufsbildenden Schulen in Deutschland auf. Hier liegen keine systematischen und repräsentativen Untersuchungen zum Grad des Einsatzes der digitalen Medien vor (vgl. BIBB 2013, 396; Eder 2015, 26ff.; Wilbers 2012, 38). Diverse Evaluationsstudien deuten zwar darauf hin, dass sich die Situation an den berufsbildenden Schulen, vor allem in der gewerblich-technischen Berufsbildung, hier etwas besser gestaltet, aber dennoch sind hier auch Gruppen von Medienbefürwortern und Medienskeptikern zu unterscheiden (vgl. Eder 2010a; 2010b). Da ein gewisser Anteil der Lehrer(-innen)kollegien an den Schulen digitale Bildungstechnologien nicht oder nur gelegentlich nutzen (vgl. Eder 2010a, 128), stellt sich die Frage, welche Faktoren eine schulische Mediennutzung bedingen bzw. wie sich der Forschungsstand dazu gestaltet und welche Maßnahmen sich daraus ableiten lassen.

\section{Bedingungsfaktoren der Akzeptanz digitaler Medien an den berufsbildenden Schulen}

Mögliche Antworten darauf bietet u.a. das Technologieakzeptanzmodell (TAM), das in den 1980er Jahren von Fred Davis erstmalig im Auftrag von IBM Kanada entwickelt wurde. Es beinhaltet Prädiktoren der individuellen Nutzungs- und Einstellungsakzeptanz von Technologien (z.B. Software) durch die Zielgruppe. Seitdem wurde das TAM von unterschiedlichen Forschern in vielfältigen Kontexten erprobt und es wurde in unterschiedlichen Varianten ausdifferenziert (z.B. TAM 1, 2 \& 3). 2003 erfolgte dann die

5 Befragungsteilnehmer(-innen) in Deutschland: 2.225 Schüler(-innen) und 1.386 Lehrer(-innen) an 142 Schulen 
Zusammenführung der unterschiedlichen Modelle durch Venkatesh, Morris, Davis und Davis (2003) in ein vereinheitlichtes Modell mit dem Akronym UTAUT „Unified Theory of Acceptance and Use of Technology“" (vgl. Venkatesh et al. 2003, 467). Dabei wurden zudem weitere Forschungsarbeiten zur Technologieakzeptanz berücksichtigt (vgl. Eder 2015, 31). Eine Adaption der TAM-Forschung auf den Bildungskontext wurde mittlerweile ebenfalls erfolgreich geleistet (vgl. Nistor et al. 2012). Im Folgenden werden die einzelnen Variablen von UTAUT und deren Zusammenhang im Überblick dargestellt und mit dem Forschungsstand zu den Bedingungsfaktoren der Nutzung digitaler Medien an den berufsbildenden Schulen (vgl. Eder 2010 a/b; 2009; 2015) abgeglichen. Ergebnis dieser Analyse sind empirisch gestützte Bedingungsfaktoren der digitalen Medienverwendung an den berufsbildenden Schulen, wobei hier die empirische Überprüfung für die berufsbildenden Schulen noch aussteht und ein Anspruch auf Vollständigkeit nicht gegeben ist.

\subsection{UTAUT im Überblick}

UTAUT ist in Abbildung 1 dargestellt und folgendermaßen aufgebaut (vgl. Eder 2015, 34): Die Absicht (Nutzungsabsicht) einer Person, eine bestimmte Technologie aufgabenbezogen zu nutzen, beeinflusst die tatsächliche Nutzung (vgl. Venkatesh et al. 2012, 178). Die Nutzungsabsicht wird dabei operationalisiert mit Items, wie: „I intend to use the system in the next (n) months "(Venkatesh et al. 2003, 460) und die tatsächliche Nutzung wird abgefragt bzw. beobachtet (vgl. Venkatesh et al. 2012, 178). In zahlreichen Regressionsstudien wurde zwischen diesen beiden Variablen ein mittelstarker Zusammenhang bestätigt $(\beta=0,40-0,60 \rho<0,001)$ (vgl. Dwivedi, Nripendra, Chen, \& Williams 2011, 164). Zudem werden sowohl die Nutzungsabsicht als auch die tatsächliche Nutzung durch unterschiedliche Variablen beeinflusst. Diese Variablen sind: (1) Leistungserwartung, (2) Anstrengungserwartung, (3) Sozialer Einfluss und (4) unterstützende Bedingungen.

Bei der Testung von UTAUT stellte sich heraus, dass die Variablen (1) Leistungserwartung, (2) Anstrengungserwartung und (3) sozialer Einfluss direkt auf die Nutzungsabsicht einwirken. Hier liegt die aufgeklärte Varianz $\left(\mathrm{R}^{2}\right)$ bei 0.7 (vgl. Venkatesh et al. 2003, 425). Die Variable (4) unterstützende Bedingungen wirkt dagegen direkt auf die tatsächliche Nutzung ein. Hier liegt die aufgeklärte Varianz bei $\mathrm{R}^{2}=0.5$ (vgl. ebenda 2003, 466). Moderiert werden die Zusammenhänge von Faktoren, wie Alter, Geschlecht, Erfahrung im Umgang mit der Technologie und Freiwilligkeit der Nutzung (vgl. Eder 2015, 34). Die interne Konsistenz der Skalen (Cronbachs Alpha) liegt zwischen 0,77 und 0,94 (vgl. Venkatesh et al. 2003, 464). 
Abb. 1: UTAUT-Modell

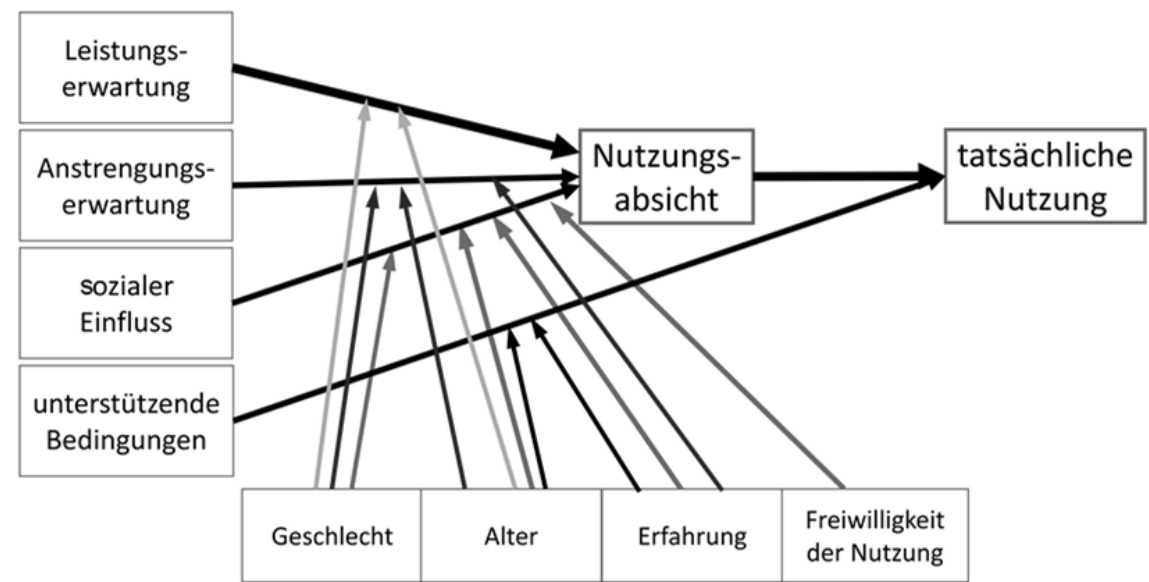

Quelle: vgl. Venkatesh et al. 2003, 447, eigene Übersetzung

\subsection{Bedingungsfaktoren der Technologie-Akzeptanz und vergleichende Betrachtung zur Situation an (berufsbildenden) Schulen}

In diesem Kapitel werden nun die einzelnen Prädiktoren der tatsächlichen Nutzung und der Nutzungsabsicht der Technologieakzeptanzforschung im Überblick vorgestellt. Zudem erfolgt ein Abgleich mit den verfügbaren Daten, welche die Akzeptanz digitaler Medien an Schulen betreffen.

Die Variable unterstützende Bedingungen wird zuerst vorgestellt, da diese direkt auf die tatsächliche Nutzung einwirkt. Sie misst, inwieweit eine Person wahrnimmt, ob eine organisatorische und technische Infrastruktur existiert, die die Nutzung eines technischen Systems unterstützt. Die Items zur Operationalisierung zielen dabei auf mehrere Aspekte ab: „I have the resources necessary to use the system. I have the knowledge necessary to use the system. A specific person (or group) is available for assistance with system difficulties." (Venkatesh et al. 2003, 460). Damit wird zum einen auf die Ressourcenfrage (Zeit, Equipment, Medienkompetenz) und zum anderen auf die Supportstrukturen eingegangen. In unterschiedlichen Studien zeigte sich hier, dass die Variable unterstützende Bedingungen vor allem bei älteren Personen einen Einfluss auf die tatsächliche Nutzung ausübt $\left(\beta=0,17-24^{*}, \rho<0,05\right.$, vgl. ebd. 2003, 466). Unterschiedliche qualitative Befragungen an berufsbildenden Schulen $\left(n_{\text {Gesamt }}=5540\right)$ und die Ergebnisse der ICILS-Studie 2013 belegen in diesem Zusammenhang, dass die fehlende IT-Ausstattung, der Mangel an Medienkompetenzen bzw. an medienpädagogischen Kompetenzen, die temporären technischen Störungen sowie der kaum verfügbare technische bzw. pädagogische Support häufig als hemmende Faktoren für die Nutzung von 
digitalen Medien im Unterricht von Lehrkräften benannt werden. Ebenso wird die ITInfrastruktur im Hinblick auf die Verfügbarkeit und Zugänglichkeit (Abschottung in Computerräumen) von Soft- und Hardware (Computer, Whiteboards etc.) als magelhaft beurteilt (vgl. Gerick, Schaumburg, Kahnert, \& Eickelmann 2014, 160ff.). Die defizitäre IT-Ausstattungssituation und der mangelnde IT-Service und -Support werden auch in Studien, die berufsbildenden Schulen betreffend, in der Regel beklagt (vgl. Eder 2010b, 155; Jepsen 2014, 78ff.).

Damit geht natürlich auch die Variable Anstrengungserwartung einher, die misst, inwieweit die Nutzung einer Technologie als einfach empfunden wird bzw. inwieweit der zugehörige Kompetenzerwerb sich einfach gestaltet. Die Operationalisierung erfolgt durch Items, wie: „It would be easy for me to become skillful at using the system. I would find the system ease to use." (Venkatesh et al. 2012, 178). Hier zeigte sich, dass der Zusammenhang der Anstrengungserwartung durch Faktoren, wie Alter, Geschlecht und Nutzungserfahrung, moderiert wird und diesbezüglich schwachmittlere Zusammenhänge $(\beta=0,16-0,21)$ festgestellt werden (vgl. Dwivedi et al. 2011, 166; Taiwo \& Downe 2013, 53; Venkatesh et al. 2012, 168). Unterschiedliche qualitative Befragungen an berufsbildenden Schulen $\left(n_{\text {Gesamt }}=5540\right)$ verdeutlichen in diesen Zusammenhang vor allem die Bedeutung der Medienkompetenz bzw. der medienpädagogischen Kompetenzen (vgl. Eder 2015, 37). Hier gibt mehr als 1/3 der Lehrkräfte an berufsbildenden Schulen an, dass diese zu wenig ausgeprägt sind und die fehlenden Medienkompetenzen den digitalen Medieneinsatz an den Schulen hemmen (vgl. Eder 2010b, 155). Weitere Korrelations-/Regressionsstudien belegen hier mittelstarke Zusammenhänge zwischen selbsteingeschätzter Medienkompetenz und angegebener Nutzung digitaler Medien $\left(r_{(\mathrm{n}=191)}=0,63(p<0,01)\left(\mathrm{vgl}\right.\right.$. Eder 2009, 232) \& $r_{(\mathrm{n}=720)}=0,59(p<0,01)(\mathrm{vgl}$. Prasse 2012, 165), $\beta=0,35$ ( $\mathrm{p}<0,05)$ (vgl. Eickelmann et al. 2014, 210).

Ebenfalls einen sehr starken Prädiktor stellt die Leistungserwartung dar (vgl. Eder 2015, 37). Diese Variable misst, inwieweit eine Person erwartet, dass die Nutzung der Technologie zu einer aufgabenbezogenen Leistungssteigerung führt bzw. aus ihr anderweitige Gratifikationen resultieren (vgl. Venkatesh et al. 2012, 159). Die Operationalisierung geschieht durch Items, wie: Using the system increases my productivity. If I use the system, I will increase my chances of getting a raise." (Venkatesh, et al. 2003, 460). Metaanalysen zur Technologieakzeptanzforschung bestätigen, dass der Regressionskoeffizient bezüglich des Zusammenhangs zwischen Leistungserwartung und Nutzungsabsicht im Durchschnitt stark ausgeprägt $(\beta=0,54)$ und hoch signifikant $(\rho<$ 0,01 ) ist (vgl. Dwivedi et al. 2011, 166; Taiwo \& Downe 2013, 54). Vor allem bei jüngeren Männern tritt dieser Zusammenhang noch deutlicher hervor (vgl. Venkatesh et al. 2003, 467f.). Im schulischen Kontext (in Gymnasien, berufsbildenden Schulen) zeigt sich diesbezüglich ebenfalls, dass in unterschiedlichen empirischen Studien die positive Einstellung zur Wirksamkeit digitaler Medien in mittelhohem Maße mit der angegebenen Computernutzungshäufigkeit korreliert $(r=0,47-0,56, p<0,001)$ (vgl. Eder 2015, 38). Im Hinblick auf die klare Relevanz der Leistungserwartung für die Ausbildung einer Nutzungsabsicht erscheint es wesentlich, die ambivalente Befundlage zur Wirksamkeit digitaler Medien für den Lehr-Lernprozess differenziert - mit Blick auf die 
Zielgruppe, die intendierten Lernziele und das eingesetzte digitale Medium - darzustellen. Hier stellt sich überdies auch die Frage, ob immer ein angemessenes Forschungsdesign den Ergebnissen zugrunde liegt (vgl. Eder 2009, 79). Beispielsweise kann die Wirksamkeit digitaler Medien nicht immer auf das Abschneiden in Schulleistungstests reduziert werden, wenn eigentlich andere Lernziele mit dem Medieneinsatz erreicht werden sollen, z.B. Selbstreflexionsfähigkeit durch E-Portfolio oder der Erwerb von Handlungskompetenzen im Umgang mit bestimmten Medien. Unterschiedliche qualitative Befragungen an berufsbildenden Schulen $\left(n_{\text {Gesamt }}=5540\right)$ ergaben diesbezüglich, dass für viele Lehrkräfte an berufsbildenden Schulen der Mehrwert einer digitalen Mediennutzung nicht klar ist und dass sie häufig die Auffassung vertreten, dass mit traditionellen Medien die intendierten Lernziele gleichermaßen erreicht werden können (vgl. Eder 2010b, 155). Die kontroverse Befundlage zur Wirksamkeit von z.B. computergestütztem Unterricht (siehe Kapitel 2) oder webbasiertem Lernen trägt das Übrige dazu bei. Es ist nicht davon auszugehen, dass die Lehrkräfte im Alltagsgeschäft die Muse finden, empirische Studien differenziert zu analysieren und einzuschätzen (vgl. Eder 2015, 38).

Ein weiterer Prädiktor, der die Nutzungsabsicht betrifft, ist abschließend die Variable sozialer Einfluss. Sie gibt an, in welchem Maße eine Person denkt, dass z.B. Vorgesetzte oder Kolleg(-innen) eine Systemnutzung erwarten (vgl. Nistor et al. 2012, 349). Operationalisiert wird der soziale Einfluss mit Items, wie: „People who are important to me think that I should use the system. In general, the organization has supported the use of the system."(Venkatesh et al. 2003, 460). Im Rahmen der Akzeptanzforschung wurde festgestellt, dass erst dann ein signifikanter Einfluss der Variablen festzustellen ist, wenn die Nutzung der Technologie vorgeschrieben wird und ältere Arbeitnehmer unter sozialen Druck gesetzt werden (vgl. Venkatesh et al. 2003, 466). ${ }^{6}$ Im schulischen Kontext belegt die ICILS-Studie, dass an den Schulen, an denen die Lehrkräfte wahrnehmen, dass der Einsatz von Bildungstechnologien Priorität hat, diese auch häufiger im Unterricht genutzt werden $\beta(\mathrm{n}=2.225)=0,2(p<0,05)($ vgl. Eickelmann et al. 2014, 210). So liegt die These nahe, dass eine hohe Erwartungshaltung - seitens der Schulleitung, der Kolleg(-innen), der Betriebe oder der Schüler(-innen) - digitale Medien im Unterricht zu nutzen, die Wahrscheinlichkeit einer verstärkten Nutzung im Unterricht zumindest geringfügig erhöht (vgl. Eder 2015, 40).

\section{Handlungsempfehlung und kritisches Resümee}

Zusammenfassend lässt sich konstatieren, dass mit der Integration digitaler Medien in berufsbildende (und auch in allgemeinbildende) Schulen sowie in die Aus- und Weiterbildung eine hohe Erwartungshaltung verbunden ist. Seitens der Bildungspolitik wird

$6 \quad(\beta$ (Geschlecht $\mathrm{x}$ Alter $\mathrm{x}$ Freiwilligkeit berücksichtigt $) \mathrm{T} 1-\mathrm{T} 3=0,16-0,27, \rho($ Geschlecht $\mathrm{x}$ Alter $\mathrm{x}$ Freiwilligkeit berücksichtigt)T1-T3 $<0,05$ ). 
eine adäquate Medienkompetenzförderung im Hinblick auf die Entwicklung von arbeitsmarktrelevantem Medienwissen, kompetentem Medienhandeln und reflektierter Medienbewertung von Heranwachsenden und Erwachsenen im Bildungs- und Beschäftigungssystem gefordert. Eine kompetente Nutzung digitaler Medien wird - so weisen es z.B. die internationale ICILS-Studie von 2013 oder die JIM-Studie 2015 nach - jedoch z.B. von Heranwachsenden noch nicht aufgrund der hohen Verfügbarkeit und Zugänglichkeit aus eigener Motivation heraus praktiziert, sondern die umfassend medienkompetente und lebensdienliche Handhabung muss erst erlernt werden. Hier muss die allgemeine und berufliche Bildung ihre Funktion wahren. In Deutschland wird die Medienintegration an den allgemeinbildenden Schulen jedoch aktuell als defizitär und nicht den Ansprüchen genügend eingestuft und für die Nutzung an den berufsbildenden Schulen liegt ein klares Forschungsdesiderat vor. Vor diesem Hintergrund wird in diesem Beitrag die Frage gestellt, welche Bedingungsfaktoren es zu verändern gilt, um die Nutzung an berufsbildenden Schulen möglichst unterstützend zu gestalten. Hierzu haben einige nicht repräsentative Evaluationsstudien an berufsbildenden Schulen, repräsentative Studien an allgemeinbildenden Schulen sowie die Technologie-Akzeptanzforschung (hier UTAUT) wichtige und sich nicht widersprechende empirische Ergebnisse erbracht (vgl. Eder 2010b, 157; Petko 2012), erheben jedoch auch keinen Anspruch auf Vollständigkeit. Die Ergebnisse deuten darauf hin, dass vor allem die Faktoren (1) unterstützende Bedingungen (2) Anstrengungserwartung (3) Leistungserwartung, und (4) sozialer Einfluss in der Wahrnehmung von Lehrkräften förderlich gestaltet werden müssen. Es gilt, eine leistungsfähige berufsschulische IT-Infrastruktur (z.B. WLAN, mobile bzw. stationäre Endgeräte im Klassenzimmer, Whiteboards, technische Lernsysteme etc.) inklusive Supportsystem, d.h. Wartung, zur Verfügung zu stellen. Digitale Endgeräte dürfen nicht in Computerräumen abgeschottet werden, sondern es muss gewährleistet werden, dass sie situationsadäquat und in angemessener Anzahl zugänglich sind. Es gilt, entsprechende Software (z.B. Learning Management Systeme, WBTs etc.) ebenfalls bereitzustellen, deren Umgang und didaktische Implementierung in den Unterricht und zur Vor- und Nachbereitung des Unterrichts geschult werden. Dies ist mit umfänglichen Kosten verbunden. Soll eine möglichst weitreichende und facettenreiche Integration digitaler Medien gewährleistet werden, ist die Schaffung dieser „unterstützenden Bedingungen“ jedoch unvermeidlich. Die Zugänglichkeit wird so gewährleistet und technische Probleme können schneller gelöst werden. Dadurch reduziert sich die Anstrengungserwartung der intendierten Nutzer. Im Hinblick auf diesen Faktor ist auch die Kompetenzentwicklung der Lehrkräfte von entscheidender Bedeutung. Die diesbezügliche Situation erweist sich laut Wilbers im Bundesgebiet als uneinheitlich und ist, was die formelle und informelle Kompetenzentwicklung in der ersten, zweiten und dritten Phase der Lehrerbildung betrifft, teils als vorbildlich - teils als verbesserungswürdig einzustufen (vgl. Wilbers 2012, 39). Hier lassen sich folglich auch noch Verbesserungsbereiche und Maßnahmen je nach Standort und Bundesland spezifisch identifizieren, z.B. erscheint eine Stärkung der beruflichen Fachdidaktiken durch Professuren erforderlich, um hier fachdidaktische Medienforschung in größeren Umfang zu ermöglichen. 
Um die Leistungserwartung der Lehrkräfte zu erhöhen, gilt es, vonseiten der Schulleitung klare Zielvorgaben zur Nutzung digitaler Medien zu formulieren. Der Einbezug möglichst vieler Kolleginnen und Kollegen erhöht zusätzlich den sozialen Aufforderungscharakter. Ebenso müssen Überlegungen angestrengt werden, wie eine Nutzung digitaler Medien in Teilbereichen auch zu einer Zeit- und Arbeitsersparnis führen kann. Hier stehen von unterschiedlichen Verlagen und Firmen auch bezahlbare Angebote zur Verfügung, die je nach den Rahmenbedingungen und den didaktischen Orientierungen der Lehrkraft verwendet werden können, ohne dass erst ein eigener Medieninhalt zeitaufwendig erarbeitet werden muss. Es gilt, Angebote hierzu für jeden Bildungsgang bzw. Ausbildungsberuf zu recherchieren. Auch eine überregionale Zusammenarbeit von Bildungsgangteams würde sich hier anbieten, um Synergieeffekte zu erzielen. Vielfältige Möglichkeiten dazu sind gegeben, insofern ist ein realistischer Nutzen zu erwarten, der damit verbundene Aufwand vertretbar und ein Prestigegewinn bei Vorgesetzten, Kolleg(inn)en, Auszubildenden oder den betrieblichen Partnern zu erwarten ist. Wie stark sich diese Bedingungsfaktoren auswirken hängt auch von Moderatorvariablen, wie Alter, Geschlecht, Computerwissen, ab. Sehr selbstsichere und computererfahrene Personen mit hohem Computerwissen werden in der Regel weniger stark durch ungünstige Rahmenbedingungen in ihrer Computernutzung negativ beeinflusst als z.B. ältere, computerängstliche Personen mit geringerer Erfahrung (vgl. Nistor et al. 2012, 348).

Abschließend bleibt noch darauf zu verweisen, dass das UTAUT-Modell zwar in vielfältigen Kontexten erfolgreich erprobt wurde - auch in pädagogischen - dass jedoch z.B. kritisiert wird, dass wichtige Variablen, die in anderen Technologie-AkzeptanzModellen (z.B. TAM 2, TAM 3 etc.) mitunter stärkere Beachtung finden, zu wenig herausgestellt werden, z.B. Computerängstlichkeit. Ebenso spielt der kulturelle Kontext laut Nistor eine wichtige Rolle, der kaum thematisiert wird, da das Modell überwiegend im anglo-amerikanischen Raum eingesetzt wurde. In den Bildungskontext sollten noch weitere Variablen aufgenommen werden, die von entscheidender Bedeutung sind, z.B. die Fähigkeit zum selbstgesteuerten Lernen (vgl. Nistor et al. 2012, 351f.). Trotz dieser Limitationen bietet das Modell eine gute Grundlage, die auch für die Erforschung der Technologie-Akzeptanz in der beruflichen Bildung adaptiert werden kann. Ein Forschungsprojekt dazu ist aktuell noch nicht terminiert. Insgesamt ist ein klares Forschungsdesiderat zur Nutzung digitaler Medien an berufsbildenden Schulen festzustellen (vgl. Petko 2012, 46). Repräsentative Studien in diesem Bereich sind notwendig, um den aktuellen Sachstand zur didaktischen Integration digitaler Medien im beruflichen Unterricht einschätzen zu können.

\section{Literatur}

Baacke, D. (2004). Medienkompetenz als zentrales Operationsfeld von Projekten. In S. Bergmann, J. Lauffeur, L. Mikos, G. Thiele \& D. Wiedemann (Hrsg.), Medienkompetenz - Modelle und Projekte, (S. 21-25). Bonn: Bundeszentrale für politische Bildung. 
BIBB (2013). Datenreport zum Berufsbildungsbericht 2013 - Informationen und Analysen zur Entwicklung der beruflichen Bildung. Bonn: Bundesinstitut für Berufsbildung (BIBB).

Bos, W.; Eickelmann, B., Gerick, J., Goldhammer, F., Schaumburg, H., Schwippert, K., Senkbeil, M., Schulz-Zander, R. \& Wendt, H. (2014). ICILS 2013 - Computer- und informationsbezogene Kompetenzen von Schülerinnen und Schülern in der 8. Jahrgangsstufe im internationalen Vergleich. Münster/New York: Waxmann.

Conrad, M. \& Schumann, S. (2015). Tablet-PCs im Wirtschaftsunterricht und die Rolle der Lehrperson. In J. Seifried, S. Seeber \& B. Ziegler (Hrsg.), Jahrbuch der berufs- und wirtschaftspädagogischen Forschung 2015. Schriftenreihe der Sektion Berufs- und Wirtschaftspädagogik, (S. 129-140). Opladen: Barbara Budrich.

de Witt, C. (2013). Vom E-Learning zum Mobile Learning - wie Smartphones und Tablet PCs Lernen und Arbeit verbinden. In C. de Witt \& A. Sieber (Hrsg.), Mobile Learning. Potenziale, Einsatzszenarien und Perspektiven des Lernens mit mobilen Endgeräten, (S. 13-26). Wiesbaden: Springer VS.

Dwivedi, Y. K., Nripendra, P. R., Chen, H. \& Williams, M. D. (2011). A Meta-analysis of the Unified Theory of Acceptance an Use of Technology (UTAUT). In M. Nüttgens, A. Gadatsch, K. Kautz, I. Schirmer \& N. Blinn (Hrsg.), Governance an Sustainability in Information System. Managing the Transfer and Diffusion of IT, (S. 155-170). Heidelberg: Springer.

Eder, A. (2009). Integration digitaler Medien an berufsbildenden Schulen - Eine allgemeine empirische Standortbestimmung und qualitative Studie zur Verwendung einer Computerneuausstattung an berufsbildenden Schulen. Göttingen: Sierke Verlag.

Eder, A. (2010a). Bedingungsfaktoren der Nutzung digitaler Medien an berufsbildenden Schulen - Eine empirische Standortbestimmung aus der Sicht von Lehrkräften (Teil 1). Die berufsbildende Schule, 62(4), 125-128.

Eder, A. (2010b). Bedingungsfaktoren der Nutzung digitaler Medien an berufsbildenden Schulen - Eine empirische Standortbestimmung aus der Sicht von Lehrkräften (Teil 2). Die berufsbildende Schule, 62(5), 154-158.

Eder, A. (2015). Akzeptanz von Bildungstechnologien in der gewerblich-technischen Berufsbildung vor dem Hintergrund von Industrie 4.0. Journal of Technical Education (JOTED), 3(2), 19-44.

Egloffstein, M., Kögler, K. \& Kärner, T. (2012). Unterrichtserleben in Notebook-Klassen. Eine explorative Studie im kaufmännischen Unterricht. In R. Schulz-Zander, B. Eickelmann, H. Moser, H. Niesyto \& P. Grell (Hrsg.), Jahrbuch Medienpädagogik 9. Qualitätsentwicklung in der Schule und medienpädagogische Professionalisierung (S. 219-241). Wiesbaden: VS-Verlag.

Eickelmann, B., Schaumburg, H., Drossel, K. \& Lorenz, R. (2014). Schulische Nutzung von neuen Technologien in Deutschland im internationalen Vergleich. In W. Bos, B. Eickelmann, J. Gerick, F. Goldhammer, H. Schaumburg, K. Schwippert, M. Senkbeil, R. Schulz-Zander \& H. Wendt (Hrsg.), ICILS 2013 - Computer- und informationsbezogene Kompetenzen von Schülerinnen und Schülern in der 8. Jahrgangsstufe im internationalen Vergleich, (S. 197229). Münster/New York: Waxmann.

Eickelmann, B., Gerick, J. \& Bos, W. (2014). Die Studie ICILS 2013 im Überblick - Zentrale Ergebnisse und Entwicklungsperspektiven. In W. Bos, B. Eickelmann, J. Gerick, F. Goldhammer, H. Schaumburg, K. Schwippert, M. Senkbeil, R. Schulz-Zander \& H. Wendt (Hrsg.), ICILS 2013 - Computer- und informationsbezogene Kompetenzen von Schülerinnen und Schülern in der 8. Jahrgangsstufe im internationalen Vergleich (S. 9-31). Münster/New York: Waxmann. 
Europäische Kommission (Hrsg.) (2010). Eine digitale Agenda für Europa. Mitteilung der Kommission an das europäische Parlament, den Rat, den europäischen Wirtschafts- und Sozialausschuss und den Ausschuss der Regionen. Brüssel, den 19.5.2010. KOM(2010)245 endgültig Online: http://eur-lex.europa.eu/LexUriServ/LexUriServ.do?uri=COM:2010:0245:FIN:DE (09.09.2015).

Europäische Kommission (Hrsg.) (2014). The International Computer and Information Literacy Study (ICILS) - Main findings and implications for education policies in Europe. Online: http://ec.europa.eu/education/library/study/2014/ec-icilsen.pdf (12.10.2015).

Gerick, J., Schaumburg, H., Kahnert, J. \& Eickelmann, B. (2014): Lehr- und Lernbedingungen des Erwerbs computer- und informationsbezogener Kompetenzen in den ICILS-2013Teilnehmerländern. In W. Bos, B. Eickelmann, J. Gerick, F. Goldhammer, H. Schaumburg, K. Schwippert, M. Senkbeil, R. Schulz-Zander \& H. Wendt (Hrsg.), ICILS 2013 - Computerund informationsbezogene Kompetenzen von Schülerinnen und Schülern in der 8. Jahrgangsstufe im internationalen Vergleich (S. 147-196). Münster/New York: Waxmann.

Grantz, T., Karges, T. \& Richter T. (2014). Kollaborative Fahrzeugdiagnose. Ein Ansatz zum Lernen im Arbeitsprozess mit Web-2.0 Technologien. lernen \& lehren, 29(114), 54-61.

Hattie, J. A. C., Beywl, W. \& Zierer, K. (2013). Lernen sichtbar machen. Überarbeitete deutschsprachige Ausgabe von ,,Visible Learning“, besorgt von Wolfgang Beywl und Klaus Zierer. Baltmannsweiler: Schneider Verlag Hohengehren.

Herzig, B. (2014). Wie wirksam sind digitale Medien im Unterricht? Gütersloh: Bertelsmann.

Howe, F. (2013). Potenziale digitaler Medien für das Lernen und Lehren in der gewerblichtechnischen Berufsausbildung.bwp@Berufs-und Wirtschaftspädagogik-online, Spezial 6 Hochschultage berufliche Bildung, Fachtagung 08, 1-15. Online: http:/www.bwpat.de/ ht2013/ft08/ howeft08-ht2013.pdf (20.02.2016).

Jepsen, M. (2014). IT-Infrastruktur und IT-Service an beruflichen Schulen. Eine große Herausforderung. lernen \& lehren, 29(114), 78-81.

Jenewein, K. (2014). Digitale Lernsysteme. Potenziale für die berufliche Bildung durch Blended Learning. lernen \& lehren, 29(114), 47-53.

MPFS (2015). JIM 2015 - Jugend, Information, (Multi-)Media. Basisstudie zum Medienumgang 12- bis 19-Jähriger in Deutschland. Stuttgart: Medienpädagogischer Forschungsverbund Südwest (mpfs).

Neumann, J. \& Ueberschaer, A. (2014). Web 2.0 in der dualen Berufsausbildung. Der OnlineAusbildungsnachweis zur Stärkung der Lernortkooperation, In H. Fischer \& T. Köhler (Hrsg.), Postgraduale Bildung mit digitalen Medien. Fallbeispiele aus den sächsischen Hochschulen, (S. 219-225). Münster u.a.: Waxmann.

Nistor, N., Wagner M. \& Heymann, J.O. (2012). Prädiktoren und Moderatoren der Akzeptanz von Bildungstechnologien. Die Unified Theory of Acceptance and Use of Technology auf dem Prüfstand. Empirische Pädagogik, 26(3), 343-371.

Petko, D. (2012). Hemmende und förderliche Faktoren des Einsatzes digitaler Medien im Unterricht: Empirische Befunde und forschungsmethodische Probleme. In R. Schulz-Zander, B. Eickelmann, H. Moser, H. Niesyto \& P. Grell (Hrsg.), Jahrbuch Medienpädagogik 9. Qualitätsentwicklung in der Schule und medienpädagogische Professionalisierung, (S. 29-50). Wiesbaden: VS-Verlag.

Pferdt, F. G., Kremer, H.-H. T. (2012). Berufliches Lernen mit Web 2.0 - Medien(entwicklungs) kompetenz und berufliche Handlungskompetenz im Duell? In R. Schulz-Zander, B. Eickelmann, H. Moser, H. Niesyto \& P. Grell (Hrsg.), Jahrbuch Medienpädagogik 9. Qualitätsentwicklung in der Schule und medienpädagogische Professionalisierung, (S. 289-307). Wiesbaden: VS-Verlag. 
Prasse, D. (2012). Bedingungen innovativen Handelns an Schulen. Münster: Waxmann.

Schorb, B. (2005). Medienkompetenz. In J. Hüther \& B. Schorb (Hrsg.), Grundbegriffe Medienpädagogik, (S. 30-37). 4. Auflage. München: Kopaed.

Schulmeister, R. (2012). Vom Mythos der Digital Natives und der Net Generation, Berufsbildung in Wissenschaft und Praxis. 41(3), 42-45.

Schütte, F. \& Mansfeld, T. (2013). Digitale Lehr-Lernmittel in der Metall- und Elektrotechnik. Fachdidaktische Relevanz, unterrichtsmethodische Reichweite. Zeitschrift für Berufs- und Wirtschaftspädagogik, 109(2), 304-316.

Senkbeil, M., Goldhammer, F., Bos, W., Eickelmann, B., Schwippert, K. \& Geric, J. (2014). Das Konstrukt der computer- und informationsbezogenen Kompetenzen in ICILS 2013. In W. Bos, B. Eickelmann, J. Gerick, F. Goldhammer, H. Schaumburg, K. Schwippert, M. Senkbeil, R. Schulz-Zander \& H. Wendt (Hrsg.), ICILS 2013 - Computer- und informationsbezogene Kompetenzen von Schülerinnen und Schülern in der 8. Jahrgangsstufe im internationalen Vergleich (S. 83-112), Münster/New York: Waxmann.

Taiwo, A. A. \& Downe, A. G. (2013). The Theory of user Acceptance and use of Technology (UTAUT): A META-Analytic review of Empirical Findings. Journal of Theoretical and Applied Information Techology. 49(1), 982-1003.

Venkatesh, V., Morris, M. G., Davis, G. B. \& Davis, F. D. (2003). User acceptance of information technology: Toward a unified view. MIS Quarterly, 27(3), 425-478.

Venkatesh, V., Thong, J. Y. L. \& Xu, X. (2012). Consumer acceptance and use of information technology: Extending the Unified Theory of Acceptance and Use of Technology. MIS Quarterly, 36(1), 157-178.

Wang, P. (2010). Chasing the hottest IT: Effects of information technology fashion on organisations. MIS Quarterly, 34(1), 63-85.

Weiss, R. (2012). Medienkompetenz als neue Kulturtechnik. Berufsbildung in Wissenschaft und Praxis. 41(3), 38-41.

Wilbers, K. (2012). Entwicklung der Kompetenzen von Lehrkräften berufsbildender Schulen für digitale Medien. Berufsbildung in Wissenschaft und Praxis. 41(3), 38-41. 



\section{Branchen- und lernortspezifische Herausforderungen beim Einsatz von Tablets in der überbetrieblichen Ausbildung der Schweizer Banken}

Stefan Kessler

\section{Einleitung}

Seit August 2012 werden in der Schweiz rund 85\% der jährlich 1.300 Lernenden ${ }^{1}$ in einer kaufmännischen Bankenausbildung zu Ausbildungsbeginn mit Tablets ausgerüstet. In den brancheneigenen überbetrieblichen Kursen zum Einsatz kommend sollen die Geräte einerseits den Lerntransfer zwischen dem Arbeitskontext und dem in den überbetrieblichen Kursen vermittelten bankfachlichen Wissen unterstützen. Die Tablets sind hierfür in ein papierloses Ausbildungskonzept eingebunden, welches den Lernenden ermöglicht, sämtliche in einer Online-Cloud gespeicherten Inhalte lernortunabhängig abzurufen oder durch neue Inhalte (z.B. die Dokumentation einer Arbeitsoder Lernaufgabe) zu ergänzen. Andererseits sollen durch den Einsatz von Tablets Fertigkeiten im Umgang mit digitalen Medien und Informationen eingeübt werden, welche über den derzeitigen Lernzielkatalog der bankkaufmännischen Grundbildung hinausgehen. Im Bereich der arbeitsbezogenen Handlungskompetenzen meint dies, das Tablet als „,künftiges Beratungsinstrument" (Seufert, Jenert \& Kuhn-Senn 2012, S. 12) in den Banken einsetzen zu können. Der Einsatz von Tablets soll darüber hinaus Selbstlern- und Medienkompetenzen der Lernenden fördern, indem es Möglichkeiten bietet, Transferaufträge vor Ort medial aufzunehmen und in einem OnlineLernjournal zu dokumentieren. Ferner kann das Tablet dazu genutzt werden, Mindmaps, Wissenslandkarten und persönliche Notizen zu erstellen und durch Ad-hoc-Internetrecherchen zu ergänzen. In der Absicht des Ausbildungszentrums geht es im Besonderen ,[...] um die Befähigung, ein digitales Medium (im konkreten Fall Tablet und PC) als Lern- und Arbeitsinstrument einzusetzen“ (Böniger 2015, S. 14). In der Durchführung wird dieses Ziel durch den konsequenten Verzicht auf den Einsatz von Papier in den Kursen verfolgt (vgl. ebd.).

1 Als „Lernende“ werden im Schweizer Berufsbildungswesen Auszubildende bezeichnet, welche in einer nachobligatorischen (dualen) Ausbildung auf Sekundarstufe II einen Beruf erlernen, der in einer Bildungsverordnung geregelt ist. Die dreijährige Bankenausbildung gehört zum kaufmännischen Berufsbild und führt zum Abschluss „Kaufmann/Kauffrau mit eidgenössischem Fähigkeitszeugnis (EFZ)“. 
Im Zuge der Umstellung auf die papierlose Ausbildung entstand seitens des Ausbildungszentrums der Bedarf, den Einsatz und die Nutzung des Tablets an den überbetrieblichen Kursen evaluieren zu lassen. Der Lehrstuhl für Berufsbildung der Universität Zürich untersucht in diesem Zusammenhang in einem dreijährigen Projekt (August 2013 bis Juli 2016) den Einsatz und die Nutzung des Tablets als Lern- und Arbeitsinstrument. Ausgangspunkt für den folgenden Beitrag ist der Befund, dass das Tablet in dem beschriebenen Setting sowohl bei den Lernenden als auch bei den sich am Ausbildungszentrum beteiligenden Banken teilweise auf nur geringe Akzeptanz (oder gar Ablehnung) stößt. Das Tablet wird ferner nur von wenigen Lernenden regelmäßig zum Lernen genutzt. Dieser Befund überrascht insofern, als dass im Rahmen einer im November 2010 durchgeführten Pilotstudie ${ }^{2}$ mit insgesamt 49 Lernenden des Ausbildungszentrums letztere den Einsatz der Tablets mehrheitlich positiv bewerteten (vgl. Seufert et al. 2012, S. 11).

Der Beitrag widmet sich der Frage nach den Gründen für die geringe Nutzung des Tablets seitens der Lernenden. Individuelle Erfahrungen und Widerstände in Bezug auf die Nutzung von in der Ausbildung eingesetzten digitalen Medien sind im Bereich der beruflichen Bildung bisher wenig erforscht. Gerade in Bezug auf das Tablet, welches primär zur kulturellen Alltagspraxis der Lernenden gehört (vgl. Pachler, Bachmair \& Cook 2010, S. 78), ist der Blick auf die Adressaten im Sinne von „Teilnehmenden“ (Schäffter 2010, S. 283) einer beruflichen Bildung für ein Verständnis der Gründe, welche zu einer Ablehnung des Tablets als Lerninstrument führen, fruchtbar. Folgt man Euler, Seufert und Wilbers (2006), so lassen sich Probleme bei der Implementierung und Umsetzung von E-Learning-Lernumgebungen in der Berufsbildung häufig auf unklare Zielsetzungen im Hinblick auf den primären Einsatzzweck der verwendeten digitalen Medien oder einen nicht erkennbaren Mehrwert der neu geschaffenen gegenüber der bisherigen Lernumgebung zurückführen (vgl. S. 446). In diesem Falle ist also zunächst nach dem Mehrwert der Tablets in der überbetrieblichen Bankenausbildung zu fragen. Darüber hinaus sind digitale Lernumgebungen oftmals auch nicht an bestehende organisationale und individuelle (Lern-)Kulturen anschlussfähig (vgl. ebd., S. 447) und erfordern ein entsprechendes Umdenken und Umlernen. Neben der Bedeutung der didaktischen Gestaltung der Lernumgebung (vgl. Hudson 2008; Jahnke 2013; Hillen 2013) rücken demzufolge auch potenzielle Veränderungen und Anpassungsleistungen auf der kulturellen und organisatorischen Ebene in den Fokus der Betrachtung. Lern- und Organisationskulturen werden hierbei im weiteren Sinne als Gewohnheiten und Einstellungen der betroffenen Gruppen (Lernende, Bildungspersonal und Mitarbeitende im Betrieb) bezeichnet, die mit einer gegenwärtigen Ausbildungs-

2 Die Pilotstudie wurde von einer anderen Institution durchgeführt. Die Ergebnisse sind nur bedingt öffentlich zugänglich. Für einen Überblick siehe: Seufert, Jenert \& Kuhn-Senn (2012). 
praxis verbunden sind (vgl. Euler et al., S. 439). Akzeptanzprobleme lassen sich schließlich dort verstärkt vermuten, wo der Einsatz unter Zwang erfolgt (vgl. Wiest 2014, S. 92).

Nachfolgend werden zunächst das papierlose Ausbildungskonzept an den überbetrieblichen Kursen und das Evaluationsprojekt kurz umrissen (Kapitel 2 und 3). Im Anschluss wird nach dem branchenspezifischen Mehrwert von Tablets in den überbetrieblichen Kursen gefragt (Kapitel 4). Diese Frage war Bestandteil von Expertengesprächen mit Berufsbildnerinnen und Berufsbildnern des Ausbildungszentrums. Aktuelle Ergebnisse aus einer Längsschnittbefragung der Lernenden zeigen weiter, dass der Einsatz von Tablets in der überbetrieblichen Ausbildung zu einer Reihe von Herausforderungen sowohl im individuellen Umgang mit den Geräten als auch im betrieblichen Kontext führt (Kapitel 5). Es stellt sich die Frage, inwiefern der mit dem Einsatz von Tablets in der überbetrieblichen Ausbildung verbundene Mehrwertgedanke seitens der Branche mit den derzeitigen betrieblichen Arbeitsplatzstrukturen vereinbar ist. Die Befunde dieses Beitrags (Kapitel 6) lassen sich zum einen als Problem konfligierender Ziele im betrieblich-überbetrieblichen Lernortverbund interpretieren, zum anderen eröffnet der Rückschluss auf (lern-) kulturelle Deutungsmuster Perspektiven für eine differenziertere Auseinandersetzung mit dem Tablet als „Lern- und Arbeitsinstrument“.

\section{Einsatz von Tablets in der überbetrieblichen Ausbildung}

Die kaufmännische Grundbildung der Schweizer Banken stellt eine von derzeit 21 Ausbildungs- und Prüfungsbranchen des kaufmännischen Berufs dar und führt zum eidgenössisch anerkannten Abschluss „Kaufmann/Kauffrau mit eidgenössischem Fähigkeitszeugnis (EFZ)“. Das auf der Sekundarstufe II angesiedelte Berufsbild ist zwar als Allbranchenlehre konzipiert, im betrieblichen und überbetrieblichen Teil sind die Ausbildungsanteile teilweise jedoch stark segmentiert (vgl. Maurer \& Pieneck 2013, S. 94). Etwa 85\% der rund 1300 Lernenden pro Jahrgang der Branche besuchen die so genannten „überbetrieblichen Kurse ${ }^{\text {‘3 }}$ in einem schweizweit an zwölf Standorten und nach einheitlichen Standards operierenden Ausbildungs- und Kurszentrum.

3 „Überbetriebliche Kurse“ werden im Schweizer Berufsbildungswesen bestimmte Anteile der beruflichen Grundbildung genannt, welche in Ergänzung zur „,dualen Ausbildung“ im Betrieb und in der Berufsfachschule ,überbetrieblich“, d. h. an einem „dritten“ Lernort zu organisieren sind. Die Kurse haben meist einführenden Charakter oder decken einen bestimmten Anteil des Basiscurriculums im jeweiligen Berufsbild ab. Gemäß dem schweizerischen Berufsbildungsgesetz sind solche Kurse für sämtliche Berufsbilder auf der Sekundarstufe II im Sinne einer ergänzenden Ausbildung obligatorisch durchzuführen (Art. 16 BBG, SR 412.10). 
2003 gegründet, ist es das Ergebnis gemeinsamer Bestrebungen der größten Schweizer Banken, ein „Kompetenzzentrum für die Nachwuchsförderung der hiesigen Banken“" (Wienröder 2013, S. 72) zu schaffen, wo der überbetriebliche Teil der Ausbildung absolviert werden soll.

Seit dem Ausbildungsjahr 2012/2013 wird allen 1.100 Lernenden pro Jahrgang jeweils am ersten der insgesamt 30 Kurstage ein Tablet als Lerngerät für den ausbildungsbezogenen und persönlichen Einsatz ausgehändigt. Der Einsatz des Tablets wird von den Lernenden an den Kursen konsequent eingefordert. Sämtliche kursbezogenen Lerninhalte (Kursunterlagen, Aufgabenstellungen und Lösungen) sind über eine Online-Lernplattform via Tablet abrufbar. Eine eigene App sorgt für einen zentralisierten Zugriff auf die Inhalte und beinhaltet ein mit der Lernplattform gekoppeltes persönliches Lerndossier, auf welchem Lernnotizen, Aufgabenlösungen sowie weitere ausbildungsrelevante Erzeugnisse und Artefakte (z.B. das Foto des Ergebnisses einer Gruppenarbeit) gespeichert und organisiert werden können. Der Zugriff auf dieses Dossier ist auch via PC zu Hause oder am Arbeitsplatz möglich. Weitere Apps wie Endnote oder Adobe PDF sind in das papierlose Kurskonzept mit eingebunden. Für eine effizientere Handhabung des Tablets in den Kursen wird den Lernenden am Kurstag jeweils eine Tastatur ausgeteilt und am Tagesende wieder eingezogen. Die Berufsbildnerinnen und Berufsbildner übernehmen jeweils in einem Team zu drei Personen und bei 48 Lernenden pro Kurs gleichsam die Rolle der Vermittlung bankfachlichen Wissens und der Lernprozessbegleitung.

\section{Das Projekt „Future Learning“}

Das Projekt „Future Learning “4 untersucht mögliche Auswirkungen des Einsatzes von Tablets auf das Lernen der angehenden Bankkaufleute. Potenzielle Veränderungen sollen auf der Ebene des Lernverhaltens und des Einsatzes von Lernstrategien festgestellt werden.

Die Auswirkungen des Tablet-Gebrauchs auf berufliche Lernprozesse sind bislang noch weitgehend ungeklärt. Neben dem allgemein fraglichen Zusammenhang zwischen Medieneinsatz und Lernerfolg (vgl. Russell 2001) und der Feststellung, dass der Einsatz mobiler Computer keine radikale Veränderung des Unterrichtsgeschehens bewirkt (vgl. Schaumburg, Tschackert, Prasse \& Blömeke 2008, S. 197) gibt es wenig empirische Evidenz auf verändertes Lernen durch den Einsatz digitaler Medien in der schulischen und

4 Das Projekt „Future Learning“ (Laufzeit: August 2013 bis Juli 2016) wird am Lehrstuhl für Berufsbildung der Universität Zürich unter der Leitung von Prof. Dr. Philipp Gonon \& lic. phil. Stefan Kessler durchgeführt und mit Mitteln des Zürcher Bankenverbands finanziert. 
beruflichen Ausbildung. Dies trifft bislang auch auf den Aufbau von Medienkompetenzen zu, welche über bloße Verbesserungen im Bereich der Bedienung hinausgehen (vgl. Welling \& Stolpmann 2012, S. 200). Über motivationssteigernde Effekte und eine generelle Akzeptanz der eingesetzten Geräte wird in Modellversuchen zwar immer wieder berichtet (vgl. Döbeli Honegger 2016, S. 65), diese erweisen sich im Zeitverlauf jedoch nicht immer als stabil und könnten aus Sicht der Lernenden beispielsweise auch als kurzfristig willkommen wahrgenommene Methodenabwechslung gedeutet werden (vgl. Conrad 2012, S. 70). Wenige Erkenntnisse bestehen indes aus Längsschnittstudien, welche die Einwirkungen des Medieneinsatzes auf das Lernverhalten und den Einsatz von Lernstrategien über einen längeren Zeitraum hinweg untersuchen. Studien zur Nutzung mobiler Geräte im Hochschulbereich geben hierzu erste Anhaltspunkte, indem auf unterschiedliche und im Zeitverlauf relativ beständige Nutzertypen hingewiesen wird (vgl. Galley, Adler \& Mayrberger 2014; Periske \& Friedrich 2016).

Die Hauptstudie des Projekts umfasst eine dreimalige Online-Befragung aller bereits mit einem Tablet ausgerüsteten Lernenden über einen Zeitraum von drei Jahren (kombiniertes Quer- und Längsschnittdesign - Befragungswellen im Mai 2014, Mai 2015, Mai 2016). ${ }^{5}$ Im Kohorten-Vergleich sollen dadurch mögliche Veränderungen im Lernverhalten und Lernstrategieeinsatz im Ausbildungsverlauf und zwischen einzelnen Jahrgangsstufen ermittelt werden. Ein zweiter Fokus des Projekts liegt auf der Nutzung und den Potenzialen des Geräts als persönliches „Lern- und Arbeitsinstrument“. Hierfür wurden zunächst im Herbst 2013 ausgewählte Kurse aller drei Lehrjahre teilnehmend beobachtet. Im Rahmen der Beobachtungen konnte auch ein Kurs besucht werden, welcher noch nach dem bisherigen Ausbildungskonzept (demnach ohne Tablets) durchgeführt wurde. Es zeigte sich, dass sich die Unterrichtskultur in den Tablet- wie auch in den bisherigen Kursen sehr stark an den zu vermittelnden fachspezifischen Lernzielen orientierte und die Unterrichtseinheiten stark standardisierten Abläufen folgten. Die Tablets wurden vorwiegend als Werkzeuge zur Texteingabe bzw. Annotation eingesetzt und stellten in diesem Sinne eher eine „Anreicherung“ der bisherigen Lernumgebung dar (vgl. Jahnke 2013, S. 4). Potenziale ließen sich insbesondere in der Entwicklung geeigneter Lernaufgaben sowie im technischen Bereich (Systemstabilität und Laufgeschwindigkeit) feststellen.

Über die Ermittlung von (didaktischen) Potenzialen hinaus hatten die Kursbeobachtungen die Funktion, relevante Themenkomplexe für die Längsschnittbefragung zu identifizieren. Beispielsweise ging aus den Beobachtungen und aus einigen Gesprächen mit Lernenden hervor, dass die Tablets ausserhalb der Kurse teilweise nur selten zum Einsatz kamen, was dazu führte,

5 Die bisherigen Rückläufe (Anzahl beendeter Umfragen im Verhältnis zur Grundgesamtheit) der ersten beiden Befragungswellen belaufen sich auf 75.3\% $(n=2497)$ für die Befragung im Mai 2014 und 70.5\% (n=2283) für die Befragung im Mai 2015. 
dass bei der Entwicklung des Online-Fragebogens auch die Nutzungskontexte ausserhalb der überbetrieblichen Ausbildung berücksichtigt wurden. Die Konstrukte „Lernverhalten“ und „Lernstrategien“ wurden auf drei Ebenen modelliert und in einem allgemeinen Fragenteil erhoben: Auf einer kognitiven Ebene (vgl. Orthmann \& Issing 2000) enthält der Online-Fragebogen Fragen zur Rezeption in zwei spezifischen Kurssituationen (Lesen eines Lehrbuchtextes und Zuhören beim Plenumsreferat) und Fragen zu Strategien der Informationsverarbeitung. Auf der Handlungsebene (vgl. Bannert 2007) sind es Fragen zu bevorzugten Lernorten, -zeiten und Sozialformen sowie Fragen zur Metakognition (z.B. Lernen planen) und zum Ressourcenmanagement (Lernatmosphäre schaffen). Auf einer emotionalen Ebene werden schließlich Aspekte der Lernmotivation und Selbstregulation (Ablenkung beim Lernen oder im Unterricht) erfasst. Neben diesem allgemeinen Fragenteil enthält der Fragebogen auch Fragen zum Potenzial des Tablets als Lerninstrument. ${ }^{6}$ Zusätzlich wurden neben Fragen zum Nutzen und zur Nutzung der Geräte im Ausbildungs- und privaten Kontext auch Fragen zu den Erwartungen an eine Tablet-basierte Ausbildung sowie Fragen zum Unterrichtserleben an den überbetrieblichen Kursen in den Fragebogen integriert. Zusätzliche Fragen mit offenem Antwortformat bieten den Lernenden die Möglichkeit, sich zu den Vor- und Nachteilen des Tablets für den persönlichen Lerneinsatz und zur Nutzung der Geräte in der Ausbildung zu äußern.

Um die Sicht der Lernenden auf das Tablet als Lerninstrument zu ergänzen und in der Annahme, dass sich der Einsatz von Tablets auch auf die Unterrichtspraxis auswirkt (vgl. Welling \& Stolpmann 2012, S. 199) wurden im April 2015 die Berufsbildnerinnen und Berufsbildner des überbetrieblichen Ausbildungszentrums schriftlich zum Einsatz der Tablets an den Kursen befragt (Rücklauf 76,4\%, n=42). Der Fokus lag neben Fragen zur Einschätzung des Nutzens von Tablets für die Ausbildung insbesondere auf Fragen zur Nutzung des Tablets für die eigene Lehrtätigkeit und zu den Auswirkungen des Tablets auf die Gestaltung und Organisation der Kurse sowie das Lernverhalten der Lernenden. Im Nachgang an diese Befragung wurden mit einigen von ihnen vertiefende Experteninterviews durchgeführt $(n=9)$.

Nachfolgend wird entsprechend der Fragestellung dieses Beitrags der branchenspezifische Mehrwert des Einsatzes von Tablets in der überbetrieblichen Bankenausbildung anhand der Interviews mit den Berufsbildnerinnen und Berufsbildnern und den Daten aus der Längsschnittbefragung aus unterschiedlichen Perspektiven beleuchtet und mögliche Erklärungen für den eingangs erwähnten Befund der verhaltenen Akzeptanz und zurückhaltenden Nutzung der Tablets im Ausbildungssystem „Bank“ diskutiert.

6 Beispielfragen: „Ich habe alle Kursunterlagen (eigene Lösungen von Aufgaben, Notizen, Fotos etc.) digitalisiert und auf dem Tablet verfügbar“ oder „Ich nutze das Tablet auch zur Prüfungsvorbereitung (z.B. für den Kurs-Kompetenznachweis)“. 


\section{Branchenspezifische Interessen am Einsatz von Tablets}

Gemäß dem Dachverband der schweizerischen Banken soll am dritten Lernort , ,...] in die Grundlagen der kaufmännischen Kenntnisse und Fertigkeiten sowie die allgemeinen Branchenkenntnisse eingeführt [werden]" (Swissbanking 2012). Damit steht die Vermittlung des bankfachlichen Grundwissens im Zentrum der überbetrieblichen Ausbildung der Branche Bank. Welche Kompetenzen und Methoden im Detail durch das Tablet gefördert werden sollen, ist aus dem aktuellen brancheneigenen Leistungszielkatalog für den betrieblichen und überbetrieblichen Teil der Ausbildung nicht abzulesen (vgl. SKKAB 2011). Aus diesem Grund haben wir in den Experteninterviews mit den Berufsbildnerinnen und Berufsbildnern des Ausbildungszentrums unter anderem die Frage gestellt, welchen Mehrwert Tablets in der überbetrieblichen Bankenausbildung bieten. Aus den Aussagen der Berufsbildnerinnen und Berufsbildern lassen sich unterschiedliche Zielbezüge ableiten, die mit dem Einsatz von Tablets verfolgt werden sollen. Im Zusammenhang mit dem auch vor der Bankenbranche nicht halt machenden Prozess der Digitalisierung wurde auf die zunehmende Bedeutung von Informationen und des Auffindens solcher hingewiesen:

17w [85]: Das Lernen, wie komme ich zu Informationen (...), wenn ich irgendwie vor einer Problemstellung stehe. Und das denke ich, das ist schon (...). Und durch das, dass wir jetzt eben so vermehrt Recherche-Aufträge eingebaut haben mit dem Tablet, das finde ich etwas Wichtiges für (...) das Arbeiten, für die Berufswelt.

5m [11]: Gut, ich meine, generell einfach die Verfügbarkeit von Unterlagen. Ist natürlich super wertvoll. Ich meine, gerade in der Bankenwelt, wo sehr viel so online verfügbar ist, kannst du diese Sachen noch (...). Das finde ich etwas, was (...) für sie auch Mehrwert generiert.

Neben einer solchen Informations(recherche)kompetenz werden speziell im Kundengespräch Vorteile eines gewandten und „modernen“ Auftretens mit dem Tablet gesehen - dies zuweilen auch aus dem praktischen Grund, ,[...] relativ viel Material mit dabei zu haben und trotzdem nicht einen Berg Broschüren mitnehmen zu müssen“ (25w [25]). Informationskompetenz und Medienkompetenz werden als spezifische Methodenkompetenzen für die künftigen Bankangestellten zunehmend wichtiger erachtet. In diesem $\mathrm{Zu}$ sammenhang wird mit dem Einsatz von Tablets auch ein gewisser ,Innovativitätsgeist' in Verbindung gebracht:

35w [74]: Von aussen her, ich sage jetzt mal, eine Bank würde man eher als traditionell bezeichnen. Aber trotzdem sehr mit dem Zeitgeist umgehen, weil sie einfach sonst nicht mehr wettbewerbsfähig ist mit all den digitalen Möglichkei- 
ten. Und durch die Methoden (...) eignet sich natürlich jemand auch so ein bisschen die Möglichkeit, oder sieht so ein bisschen, was hat man für Möglichkeiten durch das (...) Dass sie das auch ein bisschen bei uns mitbekommen. (...) Und vielleicht auch etwas innovativ wird, so ein zukünftiger Lernender. Ich sage jetzt, das „Bünzli““-Banken-Denken verlässt und nicht nur einfach (...) den einen Weg sieht.

Gerade in dieser Aussage einer interviewten Berufsbildnerin zeigt sich, dass im Tablet-Einsatz noch weitere Qualitäten als bloß die Förderung von Informationskompetenz und die Schaffung von Kundennähe transportiert werden. Die Tablets sollen auf der individuellen Ebene die Aneignung zusätzlicher Methodenkompetenzen im Bereich des Umgangs und der Bewertung von Informationen fördern und neue Einstellungen zur digitalen Arbeitsorganisation herausbilden. Andererseits repräsentiert der Einsatz der Geräte in der Bankenausbildung gegen außen hin das Bild einer dynamischen und mit dem Zeitgeist gehenden Branche.

\section{Akzeptanzprobleme und Diskrepanzen zur betrieblichen Ausbildung}

Die Berufsbildnerinnen und Berufsbildner des Ausbildungszentrums sehen im Einsatz von Tablets in der überbetrieblichen Ausbildung also durchaus einen Mehrwert für die Lernenden der Branche - wenn auch diese Ansicht von den Lernenden nicht in gleichem Ausmaß geteilt wird. Unter den diesjährig befragten Lernenden sehen nur gerade 30\% einen „eher großen“ bis „sehr großen“ Nutzen des Tablets für den ausbildungs- und berufsbezogenen Einsatz, wohingegen etwa $40 \%$ der Lernenden den diesbezüglichen Nutzen eher bis sehr gering einschätzen. ${ }^{8}$ Diese Prozentzahlen beziehen sich auf jene 2.283 Lernenden aus der gesamten Schweiz, welche den diesjährigen OnlineFragebogen ganz beendet haben. Das Tablet zum Lernen einzusetzen fällt vielen Lernenden schwer: Für die Hälfte (50\%) gestaltet sich das Lernen mit dem Tablet an den Kurstagen ineffizient und für ebenso viele (54\%) trifft die Aussage „Ich kann das Tablet erfolgreich zum Lernen anwenden“ nicht bzw. überhaupt nicht zu. Mit dem Tablet geht aufgrund langer Ladezeiten, Systeminstabilität und Mängel in der Ergonomie vieles weniger schnell von der Hand. Über 70\% der Lernenden stimmen der Aussage „Mit dem Tablet brauche ich für dieselben Sachen länger“ eher bzw. völlig zu. Die Lernenden

7 „Bünzli“ ist im Schweizerdeutschen ein abwertender Ausdruck für einen Spießbürger.

8 Die hier berichteten Ergebnisse basieren auf 5-Stufen-Likert-skalierten Items. Die Prozentwerte beziehen sich jeweils zusammengenommen auf die beiden oberen bzw. unteren Ausprägungen. 
haben die während der Kurse erstellten Notizen, Aufgabenlösungen, Fotos und weitere Artefakte zwar mehrheitlich digitalisiert und auf dem Tablet verfügbar $(61 \%)$ - für einen Viertel $(28 \%)$ trifft dies jedoch nicht zu. Eine Gewöhnung an den Umgang mit dem Tablet realisiert sich für einige (40\%), scheint jedoch für viele Lernenden nicht einzutreten (33\%). So setzt denn auch fast jeder zweite Lernende (48\%) das Tablet im Rahmen der Ausbildung eher bzw. sehr ungern ein.

Die geringe Freude am ausbildungsbezogenen Umgang mit dem Tablet steht ganz im Gegensatz zur hohen Vorfreude der Lernenden auf das Tablet vor Beginn ihrer Ausbildung. Es zeigt sich eine durchwegs hohe Eingangserwartung an den Einsatz des Tablets in der Ausbildung: Über drei Viertel (83\%) der Lernenden des jüngsten Jahrgangs reagierten „eher positiv“ bis „,sehr positiv“, als sie zum ersten Mal hörten, dass sie für die überbetrieblichen Kurse ein Tablet erhalten werden. Im Gegensatz dazu gab nur ein knappes Drittel (33\%) an, zum aktuellen Zeitpunkt „eher gern“ bis „sehr gern" mit dem Tablet zu lernen oder zu arbeiten. Die anfänglich hohen Erwartungen an den Einsatz eines Tablets in der Ausbildung scheinen sich im weiteren Verlauf der Ausbildung also für viele Lernenden nicht zu erfüllen.

Gründe für die verhaltene Akzeptanz des Tablets bei den Lernenden lassen sich aus den Antworten auf eine offene Frage zu den Vor- und Nachteilen des Tablet-Einsatzes für das eigene Lernen ableiten, welche im OnlineFragebogen beantwortet werden konnte. Tabelle 1 auf der nächsten Seite zeigt eine Teilauswertung der im Mai 2015 erhobenen Antworten von Lernenden im ersten und dritten Lehrjahr. Die meistgenannten Nachteile ließen sich der induktiv aus den Freitextantworten abgeleiteten Kategorie „mangelnde Effizienz im Umgang mit dem Tablet" zuordnen, was hauptsächlich der langsamen und verzögerungsreichen Hard- und Software der abgegebenen Tablets angelastet wurde. Als nachteilig wurden ebenfalls viele kleinere technische Probleme genannt, die den Kursalltag stören. Darunter fallen die technische Instabilität der verwendeten Apps und die teilweise nicht funktionierende Anbindung an die Online-Cloud. Bei den fünf meistgenannten Vorteilen wurden allen voran die Handlichkeit des Geräts (es ist klein und „handlich") und die praktische Materialorganisation (sämtliche Materialien sind immer mit dabei, kein Bücherschleppen) hervorgehoben. Interessanterweise taucht das „Papier“ als analoge Gegenfigur zum Tablet sowohl unter den Vorteilen als auch Nachteilen auf. 
Tab. 1: Vor- und Nachteile des Tablets aus Sicht der Lernenden

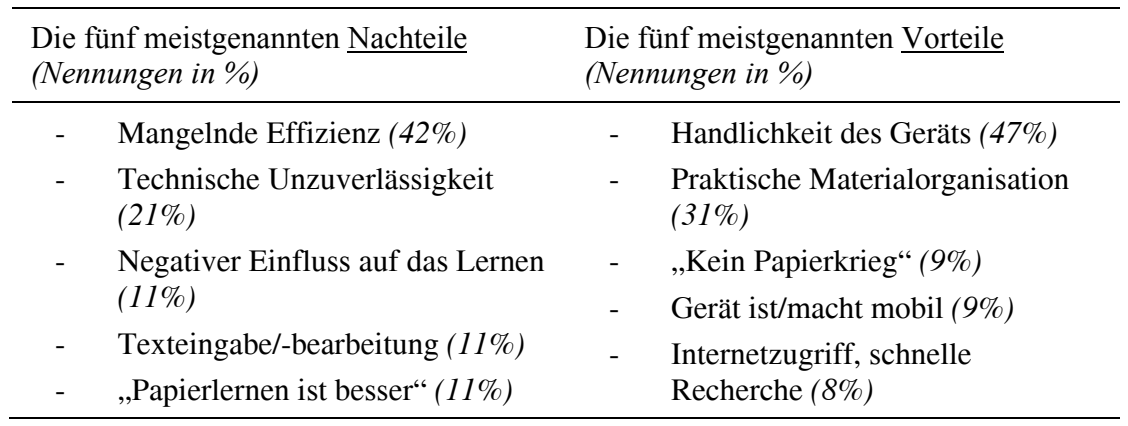

Quelle: eigene Darstellung; induktive Kategorienentwicklung (vgl. Mayring 2000) auf Basis der Lernenden-Befragung im Jahr 2015; kumulierte Teilauswertung der Antworten von Lernenden im ersten $(n=610)$ bzw. dritten $(n=454)$ Lehrjahr

Weitere Analysen machen neben der weiter oben erwähnten Diskrepanz zwischen den hohen Eingangserwartungen und der aktuellen Freude im Umgang mit dem Gerät (1) und neben den soeben aufgeführten technischen Begrenzungen des Tablets für einen effizienten Ausbildungsgebrauch (2) drittens auch auf ein mögliches Lernortproblem aufmerksam. Die zweite Online-Befragung der Lernenden bestätigt den Befund, der bereits aus der ersten Befragung hervorgegangen ist, nämlich dass das Tablet am Arbeitsplatz in den Banken selbst nur selten eingesetzt wird (s. Abb. 1) - ausgerechnet an jenem Lernort also, für dessen Einsatzfeld dem Tablet seitens der Branche hohes Potenzial zugesprochen wird. ${ }^{9}$

Ausgehend von der Annahme, dass der Lernort „Betrieb“ für die vorgefundenen Akzeptanzprobleme des Tablets eine nicht unwesentliche Bedeutung hat, konnten die Lernenden in der diesjährigen Befragung in einer offenen Frage dazu Stellung nehmen, ob und für welche Zwecke sie das Tablet auch am Arbeitsplatz in der Bank einsetzten. 1924 (84.3\%) der 2.283 ausgefüllten Fragebögen enthielten Äußerungen zu dieser Frage. Die große Mehrheit beantwortete die Frage mit einem „nein“. ${ }^{10}$ Tabelle 2 gibt einen Überblick über die Gründe, die diesbezüglich angeführt wurden. Lernende, welche das Tablet auch am Arbeitsplatz nutzen, tun dies dennoch überwiegend selten und ausschließlich für bestimmte Zwecke. In diesem Zusammenhang wurden etwa kursbezogene Lernaktivitäten wie das Bearbeiten von Kursaufträgen

9 Es zeigt sich jedoch, dass die Lernenden des jüngsten Jahrgangs (LLD14) das Tablet etwas öfter an den anderen beiden Lernorten einsetzen (s. Abb. 1). Im Hinblick auf die dritte Befragungswelle wird zu prüfen sein ob damit eine Veränderung im Nutzungsverhalten angezeigt ist oder ob die Nutzungshäufigkeit im Laufe der Ausbildung abnimmt.

10 Teilweise auch mit einem dezidierten „Nein!“ (mit Ausrufezeichen). 
oder die Lektüre des E-Lehrmittels „BankingToday 2.0“ genannt. Andererseits wird das Tablet im Betriebsalltag vereinzelt auch bei Vorträgen, Events, Produktpräsentationen sowie zum Herunterladen von Factsheets und dergleichen eingesetzt.

\section{Abb. 1: Einsatzhäufigkeit des Tablets an den Lernorten, zu Hause, unterwegs}

Frage 13.2 (t2): "Wie selten/oft verwenden Sie das Tablet ...

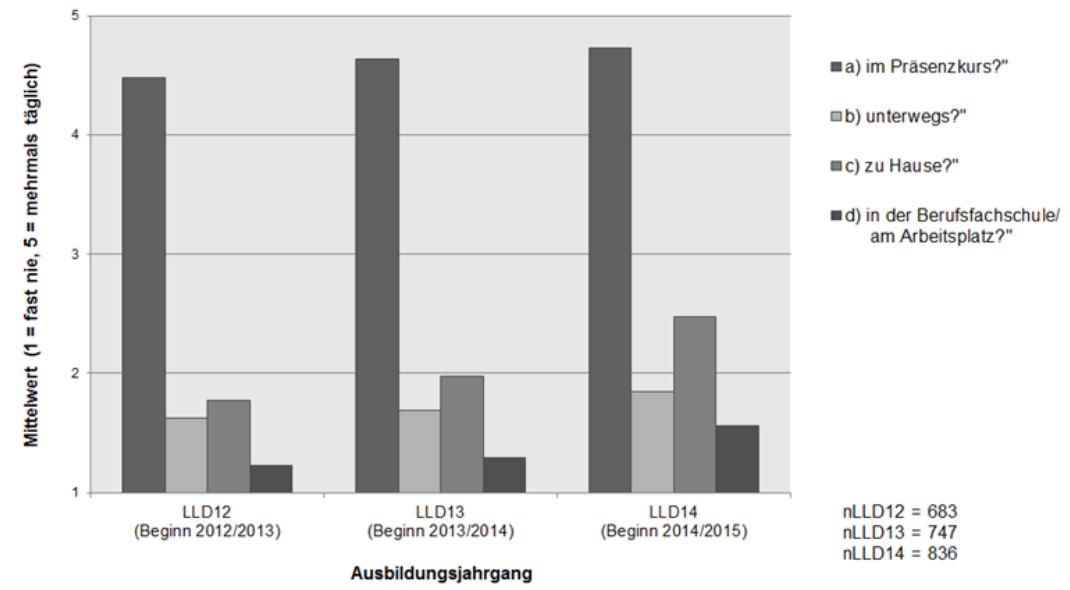

Quelle: eigene Darstellung; auf Basis der Lernenden-Befragung im Jahr 2015

Gründe für den Nicht-Einsatz lassen sich drei Ebenen zuordnen (s. Tab. 2 auf der nächsten Seite). So werden erstens wiederum technische Beschränkungen des Geräts angeführt: das Tablet sei zu langsam, eine Tastatur wäre hilfreich oder der Computer sei praktischer und besser geeignet als das Tablet. Zweitens sind die ausgehändigten Tablets teilweise mit spezifischen Arbeitsplatzstrukturen oder -kulturen nicht vereinbar. Bankeninterne Datenschutz- und Sicherheitsrichtlinien verbieten den Zugriff auf das firmeninterne Netzwerk und damit den Einsatz privater Geräte am Arbeitsort. Einige Banken verwenden in der Kundenberatung und im Empfangs-Bereich zuweilen bereits eigene Tablets, weswegen die Tablets des Ausbildungszentrums als nicht nötig erachtet werden. In den Antworten zeigt sich ferner, dass das Tablet in einigen Banken schlicht nicht erwünscht ist - auf den Kunden gebe ein am Schreibtisch sitzender, mit dem Tablet hantierender Angestellter ein unseriöses Bild ab. Darüber hinaus sei einigen (wenigen) Praxisausbildnerinnen und Praxisausbildnern im Betrieb offenbar nicht bekannt, dass am Ausbildungszentrum Tablets eingesetzt werden und dass das Tablet - Zitat eines Lernenden im dritten Lehrjahr - „das Lerninstrument der Lernenden ist“. Einer dritten Ebene lassen sich schließlich Gründe zuordnen, welche die Lernenden 
im Zusammenhang mit persönlichen Lern- und Arbeitsgewohnheiten anführen. So würden die Kursaufgaben beispielsweise lieber auf dem Computer erledigt oder das Tablet sei für den Einsatz am Arbeitsplatz ungeeignet und biete keinen Mehrwert zum Computerarbeitsplatz.

Tab. 2: Gründe für den Nicht-Einsatz des Tablets am Arbeitsplatz

\begin{tabular}{|c|c|c|}
\hline $\begin{array}{l}\text { 1. Technische } \\
\text { Beschränkungen auf } \\
\text { Geräteebene }\end{array}$ & $\begin{array}{l}\text { 2. Inkompatibilität mit } \\
\text { Strukturen und der Kultur } \\
\text { am Arbeitsplatz }\end{array}$ & $\begin{array}{l}\text { 3. Andere Lern- und } \\
\text { Arbeitsgewohnheiten }\end{array}$ \\
\hline \multirow{6}{*}{$\begin{array}{ll}\text { - } & \text { Tablet zu langsam } \\
\text { - } & \text { Tastatur wäre } \\
\text { hilfreich } \\
\text { - } & \text { Computer praktischer } \\
& \text { und besser geeignet }\end{array}$} & \multirow{4}{*}{$\begin{array}{l}\text { - } \quad \text { Tablet-Einsatz am } \\
\text { Arbeitsplatz verboten } \\
\text { (z.B. aufgrund von } \\
\text { Datenschutz oder } \\
\text { sensibler Daten) }\end{array}$} & \multirow{3}{*}{$\begin{array}{l}\text { Einsatz am } \\
\text { Arbeitsplatz }\end{array}$} \\
\hline & & \\
\hline & & \\
\hline & & \multirow{2}{*}{$\begin{array}{l}\text { Kursaufgaben } \\
\text { werden lieber auf } \\
\text { dem PC erledigt }\end{array}$} \\
\hline & $\begin{array}{l}\text { - } \quad \text { Einsatz eigener, } \\
\text { interner Tablets }\end{array}$ & \\
\hline & $\begin{array}{l}\text { Tablets nicht } \\
\text { erwünscht, akzeptiert } \\
\text { oder bekannt am } \\
\text { Arbeitsplatz }\end{array}$ & $\begin{array}{l}\text { Auf Kursunterlagen } \\
\text { kann auch via } \\
\text { Internet zugegriffen } \\
\text { werden }\end{array}$ \\
\hline
\end{tabular}

Quelle: eigene Darstellung; induktive Entwicklung der Oberkategorien (vgl. Mayring 2000) auf Basis der Lernenden-Befragung im Jahr 2015; Auswertung der Antworten von Lernenden auf die Frage ,,Setzen Sie das Tablet auch an Ihrem Arbeitsplatz ein? Wenn ja: Für welche Zwecke?"

Die Antworten der Lernenden machen deutlich, dass die Resonanz auf das Tablet auch bei den Banken (und somit am späteren Anstellungsort) selbst gemischt ist. Indizien dafür lassen sich nicht zuletzt auch im öffentlichen Auftritt der Banken festmachen, wie zwei aktuelle Internetauftritte für Ausbildungsbewerberinnen und -bewerber zur Kauffrau bzw. zum Kaufmann Bank zeigen: Im Anforderungsprofil für Lehrstellenbewerbende einer größeren Banken-Gruppe befindet sich bereits der Zusatz „Interesse am Umgang mit Online-Medien und Freude am Lernen mit dem Tablet“. In einem Lehrstellenportrait-Film einer Zürcher Großbank wird hingegen noch die „gute, alte Beratermappe“ (34m [50]) in Szene gesetzt. Dies entspricht letztlich nicht dem Bild des Tablets als „,künftiges Beratungsinstrument“ im Alltagsgeschäft der Bankenangestellten.

34m [50]: Ich bin überzeugt, ein Tablet wird die gute, alte Beratermappe verdrängen. (...) Das ist sicher, glaube ich, das wird aus meiner Sicht der Haupteinsatzort sein von so mobilen Devices. (...) Also, sprich, der Berater wird nicht mehr mit einer Riesen-Ledermappe kommen, wo er jenes an Prospekten und 
überhaupt und sowieso drin hat, sondern (...) das wird eher ein kleines, handliches Ding sein. Wo man direkt vielleicht auch animiert irgendwelche Sachen dem Kunden zeigen kann. (.) Also, ich glaube dort, das wird der Haupteinsatzort sein. Sonst im Arbeitsalltag auf der Bank, der ist ja eigentlich schon zu einem grossen Teil an Computern (...).

\section{Fazit und Ausblick}

Wie dieser Beitrag gezeigt hat, kommt dem Tablet aus den drei betrachteten Perspektiven (des brancheneigenen Ausbildungszentrums, der beteiligten Banken und der Lernenden) jeweils ein unterschiedlicher Stellenwert zu. Aus Sicht des brancheneigenen Ausbildungszentrums soll das Tablet dazu beitragen, die im Zuge der Digitalisierung und Informatisierung wichtiger werdenden Informations- und Medienkompetenzen der zukünftigen Bankenangestellten zu fördern. Der Einsatz von Tablets an den überbetrieblichen Kursen soll auf den späteren Einsatz von Tablets im betrieblichen Alltag vorbereiten - bei einigen Banken ist dies in der Kundenberatung und im Front OfficeBereich bereits heute Realität. Aus diesem Grund setzt die Branche in der überbetrieblichen Ausbildung neben dem Erwerb des bankspezifischen Grundlagenwissens mit dem Einsatz des Tablets als Lern- und (zukünftiges) Arbeitsgerät einen zusätzlichen Schwerpunkt zum bestehenden Lernzielkatalog. Dass der damit transportierte Effizienz-, Aktualitäts- und Arbeitsprozessgedanke in den Antworten der Lernenden nicht in jedweder Hinsicht abgebildet wird, zeigt die obige Darstellung. Aus Sicht der Lernenden sind technische Begrenzungen der Geräte sowie die Inkompatibilität der digitalen Lern- und Arbeitsorganisation mit den eigenen Lern- und Arbeitsgewohnheiten im Sinne unterschiedlicher Lernkulturen (vgl. Euler et al., S. 439) entscheidende Gründe für eine ablehnende Haltung gegenüber dem Tablet. Aufgrund der teilweise widersprüchlichen Botschaften, welche die Lernenden in Bezug auf die Bedeutung des Tablets an den verschiedenen Lernorten erfahren, fällt es vielen unter ihnen schwer, einen unmittelbaren Nutzen für den anwendungsbezogenen Einsatz des Tablets im betrieblichen Alltag auszumachen. Aus Sicht der Banken - deren Perspektive hier mittels Gesprächen mit den Berufsbildnerinnen und Berufsbildnern des Ausbildungszentrums sowie durch die Antworten von Lernenden erfasst werden konnte - stellt sich die Frage, wie sie sich in Zukunft gegenüber dem Tablet positionieren wollen und wie das Tablet das zukünftige Erscheinungsbild der Banken prägen wird.

Diese Befunde geben Anlass zur Diskussion. So stehen die mit der Einführung von Tablets in die überbetriebliche Ausbildung verbundenen Erwartungen der Branche in partiellem Konflikt mit den Arbeitsplatzstrukturen und -kulturen sowie dem aktuellen Erscheinungsbild der beteiligten Banken. Die 
technischen und situationsbezogenen Anwendungsgebiete der Geräte bleiben zumindest aus Sicht der Lernenden aktuell hinter deren Erwartungen und Möglichkeiten zurück. Diese Ergebnisse legen zunächst nahe, die Qualitäten des Tablets als „Lern- und Arbeitsinstrument“ in Abhängigkeit des jeweiligen Anwendungsgebiets zu bestimmen und im Vorfeld durch eine Bedarfsund Zielgruppenanalyse genau zu eruieren. Gleichwohl bedürfen die dargelegten Befunde aber auch nach mittelbareren Erklärungen.

Ins Licht rücken zweitens Probleme der Lernortkooperation aufseiten der Anbieter beruflicher Bildung und damit Probleme, welche in der Konzeption der überbetrieblichen Ausbildung selbst angelegt sind. Die Lancierung eines „dritten Lernorts“ in die duale Ausbildung macht die Koordination und den Transfer zwischen den Lernorten zu einer komplexen Aufgabe, welcher sich die an der Ausbildung und der Definition der Ausbildungsinhalte beteiligten Akteure stellen müssen. Durch eine zu nahe Angliederung an den einen oder anderen Lernort wird die „Erfüllung der Transferaufgabe“" (Landwehr 2002, S. 63) des dritten Lernorts zusätzlich erschwert (vgl. ebd.). Die Lernortkooperation könnte gestärkt werden, indem das Bildungspersonal in den Banken verstärkt aktiv in die Gestaltung der virtuellen Lerninfrastruktur einbezogen wird und diese nutzt (vgl. Zinke 2008, S. 24). Der Einsatz von Tablets führt drittens zum Aufeinandertreffen unterschiedlicher Lern- und Organisationskulturen und zeigt die Anwendungsmöglichkeiten, aber auch seine Grenzen im gegenwärtigen Ausbildungssystem der Schweizer Banken auf. Wenn die im Rahmen der überbetrieblichen Ausbildung angeeigneten Kompetenzen im Umgang mit dem Tablet sich im betrieblichen Kontext nicht verwerten lassen - das diesbezüglich erworbene Wissen demnach als nicht anwendbar erlebt wird (vgl. Masemann 2015, S. 2) -, verliert das Tablet in individueller Perspektive seine Verbindlichkeit als relevanter Ausbildungsgegenstand. Die Frage, inwiefern individuelle Lernstile (vgl. ebd.) und habituelles Handeln sich in der papierlosen Ausbildung entfalten können, bleibt offen. Die Verbindlichkeit des Tablet-Einsatzes sollte - so lassen sich die Ergebnisse abschließend interpretieren - hierbei nicht auf Kosten der Lernwegoffenheit erwirkt werden. Der Rückgriff auf Erklärungskonzepte, welche den Einsatz von Tablets sowohl in einer Transferperspektive betrachten (vgl. Eickelmann 2010) als auch das habituelle Handeln der Lernenden in ihren „Medienpraxiskulturen“ (Schäffer 2003) bzw. im Mediatisierungsprozess von Bildungsinstitutionen (Welling, Breiter \& Schulz 2015) berücksichtigen, erscheint für die Analyse der Dynamiken, welche durch das Tablet innerhalb des Lernortverbunds und auch im lernenden Subjekt selbst angestoßen werden, fruchtbar und im Hinblick auf weitere Untersuchungen wünschenswert.

Das Tablet ins Zentrum der Betrachtung gerückt eröffnet neue Perspektiven für ein besseres Verständnis gegenwärtiger Konflikte zwischen branchenspezifischen Interessen und ihrer Umsetzung in der beruflichen Ausbildungspraxis der Schweizer Banken. Ein vorläufiges Fazit könnte lauten, dass 
die Voraussetzungen für einen tatsächlichen Mehrwert letztlich dort gegeben sind, wo der Einsatz von Tablets anschlussfähig an die tradierte Lernkultur der Lernenden gemacht werden kann und die Geräte im betrieblichen Kontext nicht als Fremdkörper wahrgenommen werden.

\section{Literatur}

Bannert, M. (2007). Metakognition beim Lernen mit Hypermedia. Münster: Waxmann.

BBG. Bundesgesetz über die Berufsbildung vom 13. Dezember 2002, BGG, SR412.10.

Böniger A. (2015). Die Mühen der „Digital Natives“ mit dem digitalen Lernen. Panorama, 2015(1), 14-15.

Conrad, M. (2012). Untersuchung netzbasierten sozialen Lernens in der beruflichen Bildung. Unveröffentlichte Masterarbeit an der Universität Konstanz.

Döbeli Honegger, B. (2016). Mehr als 0 und 1. Schule in einer digitalisierten Welt. Bern: hep.

Eickelmann, B. (2010). Digitale Medien in Schule und Unterricht erfolgreich implementieren. Eine empirische Analyse aus Sicht der Schulentwicklungsforschung. Münster: Waxmann.

Euler, D., Seufert, S. \& Wilbers, K. (2006). eLearning in der Berufsbildung. In R. Arnold \& A. Lipsmeier (Hrsg.), Handbuch der Berufsbildung (S. 432-450). Wiesbaden: VS Verlag.

Galley, K., Adler, F. \& Mayrberger, K. (2014). Der längerfristige Einfluss von Tablets auf das Studium und die persönliche Lernumgebung Studierender. In K. Rummler (Hrsg.), Lernräume gestalten - Bildungskontexte vielfältig denken (S. 114-124). Münster: Waxmann.

Hillen, S. A. (2013). What Can Research on Technology for Learning in Vocational Educational Training Teach Media Didactics? In K. Beck \& O. ZlatkinTroitschanskaia (Hrsg.), From Diagnostics to Learning Success. Proceedings in Vocational Education and Training (S. 101-113). Rotterdam: Sense Publishers.

Hudson, B. (2008). Didaktik Design for Technology Supported Learning. In M. A. Meyer, M. Prenzel \& S. Hellekamps (Hrsg.), Perspektiven der Didaktik. ZfE Sonderheft 9/2008, (S. 139-157). Wiesbaden: VS-Verlag.

Jahnke, Isa (2013). Teaching Practices in iPad-Classrooms: Alignment of Didactical Designs, Mobile Devices and Creativity. International Journal of Mobile and Blended Learning (IJMBL), 5(3), 1-16.

Landwehr, N. (2002). Der dritte Lernort. In W. Goetze, P. Gonon, A. Gresele, S. Kübler, H. Landolt, N. Landwehr et al. (Hrsg.), Der dritte Lernort. Bildung für die Praxis, Praxis für die Bildung (S. 37-71). Bern: hep.

Masemann, M. (2015). Zur Bedeutung von Lern- und Denkstilen für die berufliche Lehr-Lern-Forschung. bwp@Berufs- und Wirtschaftspädagogik-online, Nr.28. Online: http://www.bwpat.de/ausgabe28/masemann_bwpat28.pdf (29.03.2016). 
Maurer, M. \& Pieneck, S. (2013). Die Reform von Berufsbildern in der beruflichen Grundbildung. In M. Maurer \& P. Gonon (Hrsg.), Herausforderungen für die Berufsbildung in der Schweiz (S. 81-100). Bern: hep.

Mayring, P. (2000). Qualitative Inhaltsanalyse. Forum: Qualitative Sozialforschung, 1(2), Art. 20. Online: http://www.qualitative-research.net/index.php/fqs/article/ view/1089/2384/ (28.08.2015).

Orthmann, C. \& Issing, L. J. (2000). Lernen im Internet - ein integrativer Ansatz. In W. Marotzki, D. M. Meister \& U. Sander (Hrsg.), Zum Bildungswert des Internet (S. 83-96). Opladen: Leske + Budrich.

Pachler, N., Bachmair, B. \& Cook, J. (2010). Mobile Learning. Structures, Agency, Practices. New York: Springer.

Periske, M. \& Friedrich, J. D. (2016). Lernen mit digitalen Medien aus Studierendenperspektive. Sonderauswertung aus dem CHE Hochschulranking für die deutschen Hochschulen. Berlin: Geschäftsstelle Hochschulforum Digitalisierung.

Russell, T. L. (2001). The No Significant Difference Phenomenon. Raleigh: IDEEC.

Schäffer, B. (2003). Generationen - Medien - Bildung. Medienpraxiskulturen im Generationenvergleich. Opladen: Leske + Budrich.

Schäffter, O. (2010). Teilnehmende. In R. Arnold, S. Nolda \& E. Nuissl (Hrsg.), Wörterbuch Erwachsenenbildung (S. 283-284). Bad Heilbrunn: Julius Klinkhardt.

Schaumburg, H., Tschackert, K., Prasse, D. \& Blömeke, S. (2008). Neuer Unterricht mit Neuen Medien? Ergebnisse einer Videostudie zum Einsatz mobiler Computer im Unterricht. In E.-M. Lankes (Hrsg.), Pädagogische Professionalität als Gegenstand empirischer Forschung, (S. 189-199). Münster: Waxmann.

Seufert, S., Jenert, T. \& Kuhn-Senn, A. (2012). Didaktische Potenziale des Mobile Learning für die Berufsbildung. BWP-Berufsbildung in Wissenschaft und Praxis, 41(3), 10-13.

SKKAB (2011). Leistungszielkatalog Branche Bank. Bern: Schweizerische Konferenz der kaufmännischen Ausbildungs- und Prüfungsbranchen (SKKAB).

Swissbanking (2012). Struktur der Lern- und Leistungsdokumentation Bank (LLD Bank). Baustein 6: Überbetriebliche Kurse und Kompetenznachweise. Basel: Schweizerische Bankiervereinigung. Online: http:/www.swissbanking.org/lldbank/ (03.09.2015).

Welling, S., Breiter, A. \& A. H. Schulz (2015). Mediatisierte Organisationswelten in Schulen. Wie der Medienwandel die Kommunikation in den Schulen verändert. Wiesbaden: Springer VS.

Welling, S. \& Stolpmann, B. E. (2012). Mobile Computing in der Schule - Zentrale Herausforderungen am Beispiel eines Schulversuchs zur Einführung von TabletPCs. In R. Schulz-Zahnder, B. Eickelmann, H. Moser, H. Niesyto \& P. Grell (Hrsg.), Jahrbuch Medienpädagogik 9 (S. 191-221). Wiesbaden: VS-Verlag.

Wienröder, H. (2013, 31. Januar). Papier war gestern. Handelszeitung, S. 72.

Wiest, S. (2014). Verwendung und Akzeptanz webbasierter Lernumgebungen in der beruflichen Bildung. Unveröffentlichte Masterarbeit an der Universität Konstanz.

Zinke, G. (2008). Lern- und Medienkonzepte in der (über-)betrieblichen Berufsbildung - Tradition und Wandel. In F. Howe, J. Jarosch \& G. Zinke (Hrsg.), Ausbildungskonzepte und Neue Medien in der überbetrieblichen Ausbildung (S. 13-28). Bielefeld: W. Bertelsmann. 


\title{
Struktur und Organisation berufsbegleitender MINT- Studiengänge
}

\author{
Julia K. Gronewold
}

\section{Berufsbegleitende Studiengänge im MINT-Bereich}

Mit der Öffnung der Hochschulen für beruflich Qualifizierte erfährt das berufsbegleitende Studium eine grundlegende Aufwertung. Dies zeigen steigende Zahlen von Beiträgen und Tagungen zu diesem Themengebiet (vgl. exempl. Pätzold 2011; Rein \& Kolter 2013; AG BFN 2014). Beruflich Qualifizierte kommen ohne klassische Hochschulzugangsberechtigung an die Hochschule, studieren in Teilzeit bzw. neben der Berufstätigkeit und bringen eine durch ihre Berufstätigkeit geprägte Handlungslogik und fundierte berufspraktische Erfahrungen mit. Ein Studium wird eher als Weiterqualifizierung und nicht als grundständige Ausbildung betrachtet. Vor diesem Hintergrund kann die Struktur und die Organisation eines Vollzeitstudiums nicht ohne weiteres auf ein berufsbegleitendes Studium übertragen werden: Es bedarf einer Veränderung der Struktur und Organisation des berufsbegleitendes Studiums. Entsprechend ist es das Ziel des Beitrags die Struktur und Organisation berufsbegleitender Studiengänge sowie Herausforderungen für Studiengangskoordinatoren und Lehrende zu beschreiben und zu analysieren.

Der Beitrag fokussiert berufsbegleitende Studiengänge der MINTBranche. Speziell in naturwissenschaftlichen und technischen Berufsfeldern werden partielle Fachkräfteengpässe sowie ein zukünftiger Mangel an Hochqualifizierten, vor allem Ingenieure und Ingenieurinnen, prognostiziert (vgl. exempl. Vogler-Ludwig \& Düll 2013; BMBF 2012). Zudem ist mit Blick auf den demographischen Veränderungsprozess zu konstatieren, dass sich die Sicherung der Qualifikationsbedarfe im MINT-Bereich zukünftig nicht mehr primär über die Rekrutierung von Absolventen grundständiger Studiengänge bewältigen lässt (vgl. Heublein, Richter, Schmelzer \& Sommer 2012, 16), ein berufsbegleitendes Studium somit zu einer der zentralen Weiterbildungsmöglichkeiten wird. Darüber hinaus kann festgestellt werden, dass es insbesondere im MINT-Bereich vergleichsweise wenige berufsbegleitend organisierte Studiengänge gibt (vgl. Minks, Netz \& Völk 2011).

Auf Basis empirischer Daten aus dem von der Hans-Böckler-Stiftung geförderten Projekt „Durchlässigkeit in naturwissenschaftlich-technischen (MINT-)Berufen“ wird folgenden Forschungsfragen nachgegangen: Welche Herausforderungen lassen sich für Studiengangskoordinatoren und Lehrende 
bei der Organisation und Koordination der berufsbegleitenden MINTStudiengänge feststellen (Mikroebene)? Welche Rolle spielen Kooperationen mit anderen Unternehmen und Bildungsanbietern (Mesoebene)? Inwiefern verändern sich Bildungsstruktur und -organisation (Makroebene) und welche Forderungen lassen sich daraus für die verschiedenen Ebenen ableiten?

Beruflich Qualifizierten im MINT-Bereich soll durch ein berufsbegleitendes Studium die Möglichkeit geboten werden, das branchenspezifischtechnische Erfahrungswissens, dessen Erwerb und Ausbau in der Arbeit stattfindet und im naturwissenschaftlich-technischen Bereich als besonders relevant eingeschätzt wird (vgl. Bauer, Böhle, Munz, Preiffer \& Woicke 2006, 28ff.), in Distanz zu dem direkten Arbeitsgeschehen in der Praxis zu betrachten und zu reflektieren. Dadurch können praxisbezogene Problemstellungen auf Basis wissenschaftlicher Theoriebestände reflexiv bearbeiten werden. Faktisch wird hiermit die Möglichkeit geschaffen die reflexive Handlungsfähigkeit (vgl. Dehnbostel 2007) aufrechtzuerhalten und weiterzuentwickeln. Der Einbezug des Erfahrungswissens sowie die reflexive theoriebasierte Bearbeitung von Praxisproblemen, sind daher für das berufsbegleitende Studium als zentrale Faktoren zu begreifen (vgl. Dittmann \& Gronewold 2015, 166).

In den folgenden Abschnitten werden zunächst das Untersuchungssample und das methodische Vorgehen des Forschungsprojektes dargestellt (2). Im Anschluss daran werden erste Ergebnisse zu der Struktur und Organisation berufsbegleitender MINT-Studiengänge (3) sowie Herausforderungen für Lehrende und Koordinierende Personen vorgestellt (4). Im Hinblick auf die kooperierenden Partner werden aus Sicht der Hochschulen Anforderungen und Entwicklungspotentiale diskutiert (5). Abschließend werden Thesen zur Veränderung und Weiterentwicklung der Bildungsstruktur und -organisation für berufsbegleitende MINT-Studiengänge formuliert (6). 


\section{Untersuchungssample und methodisches Vorgehen}

Die o. g. Untersuchung gliedert sich in einen quantitativen und einen qualitativen Teil. ${ }^{1}$ Ziel der quantitativen Erhebung ist eine Bestandsaufnahme berufsbegleitender Studiengänge im MINT-Bereich. Bei der qualitativen Analyse liegt der Fokus auf der vertiefenden Auseinanderstetzung mit der berufspraktischen Ausrichtung ausgewählter Studiengänge. Die Auswahl der Studiengänge erfolgt auf Basis der quantitativen Bestandsaufnahme.

Für die Bestandsaufnahme der berufsbegleitenden Studiengänge, wurden im Zeitraum Juni bis September 2014 folgende vier Aus- und Weiterbildungsdatenbanken quantitativ ausgewertet: Der „Hochschulkompass“ der HRK, die Datenbanken „studieren.de“ und „,berufsbegleitend-studieren.de“ und die ,Zentralstelle für Fernstudien an Fachhochschulen“ (ZFH). Berücksichtig wurden Studienangebote von Hochschulen und Universitäten mit dem Abschluss Bachelor of Science (B. Sc.) und Bachelor of Engineering (B. Eng.). Insgesamt konnten 77 Studiengänge gefunden werden, die berufsbegleitend studiert werden können.

Auf Basis der quantitativen Bestandsaufnahme konnten acht Studiengän$\mathrm{ge}^{2}$ für die qualitativ angelegte Erhebung der Herausforderungen für Lehrende und Studienengangskoordinatoren sowie für die Anforderungen der Kooperationspartner gewonnen werden. Dadurch ergibt sich für den qualitativen Part folgendes Untersuchungssample: Wirtschaftsinformatik $(2 \mathrm{x})$, Industriechemie (2x), Mechatronik (2x), Elektrotechnik und Energietechnik. Aus diesen Fächern wurden mit Lehrenden und Studiengangskoordinatoren leit-

1 Das Projekt „Durchlässigkeit in naturwissenschaftlich-technischen (MINT-)Berufen“ hat ein komplexes methodisches Forschungsdesgin, um dem geringen Forschungsstand in Bezug auf das berufsbegleitende Studium gerecht zu werden. Im vorliegenden Artikel wird auf erste Ergebnisse aus der bis zum Zeitpunkt der Veröffentlichung des Artikels durchgeführten und ausgewerteten Empirie zurückgegriffen. Daher wird hier nicht das gesamte empirische Vorgehen beschrieben, sondern nur die für die hier rezipierten Ergebnisse wichtigen empirischen Schritte. Es handelt sich somit um einen noch nicht vollständig durchgeführten Forschungsprozess, welcher eher der zeitlichen Bearbeitung als einem idealtypischen Forschungsverlauf folgt.

2 Die Auswahl der insgesamt acht Studiengänge orientierte sich an den für den MINT-Bereich typischen Fächern Maschinenbau, Informatik, Naturwissenschaften und Technik. Ziel war es zumindest jeweils einen Studiengang aus den entsprechenden Bereichen im Sample abzubilden. 
fadengestützte Interviews geführt. ${ }^{3}$ Die so zustande gekommenen sechzehn Interviews wurden mittels eines induktiven Analyseverfahrens (Strukturierung) inhaltsanalytisch ausgewertet (vgl. Mayring 2002). Durch induktive Kategorienbildung werden einzelne Textpassagen im analysierten Material zu thematischen Sequenzen geordnet (Kategorisierungsebene 1 - Strukturierung; z.B. Kooperation, Organisation). Im Anschluss daran werden innerhalb der thematischen Sequenzen verallgemeinerbare Muster expliziert (Kategorisierungsebene 2 - Explikation; z.B. berufspraktische Orientierung, Verzahnung von Theorie und Praxis) (vgl. ebd.).

\section{Struktur und Organisation berufsbegleitender MINT- Studiengänge}

Von den o. g. insgesamt 77 berufsbegleitenden MINT-Studiengängen sind je 37 an einer staatlichen bzw. privaten (Fach-)Hochschule angesiedelt. Auffällig ist, dass lediglich drei Studiengänge (4\%) an staatlichen Universitäten angeboten werden. In Orientierung an der Systematik des Statistischen Bundesamtes (vgl. Destatis 2013) wurden diese Studienangebote hinsichtlich ihrer fachlichen Ausrichtung unterschieden und einer der Studienfachgruppen zugeordnet: 49 Studiengänge lassen sich so in die Ingenieurswissenschaften und 28 in die Naturwissenschaften einordnen, wobei sich in den Naturwissenschaften vier Studiengänge der Chemie und 24 der Informatik zuweisen lassen.

Im Hinblick auf die branchenbezogenen Qualifizierungswege und Übergänge zwischen den Bildungssektoren ist eine hohe Komplexität und Intransparenz zu konstatieren. Beispielsweise ist ein hoher Rechercheaufwand zu betreiben, um Studiengänge zu identifizieren, die auf Basis einer Berufsausbildung zum Studium berechtigen. Diese formalrechtlichen Hindernisse, welche eine Beteiligung und den Erfolg eines berufsbegleitenden Studiums für die beruflich Qualifizierten erschweren, sind nicht zu Letzt Folge der unterschiedlichen Gesetzgebungen der einzelnen Bundesländer (vgl. exempl.

3 Die leitfadengestützte Gesprächsstrategie macht es möglich den Gegensatz zwischen formaler Interviewsituation und erwünschter Offenheit des Interviewten aufzuheben (vgl. u. a. Gläser \& Laudel 2009), indem sowohl auf die subjektive Problemsicht als auch auf die im Vorfeld erstellten Fragestellungen und Erkenntnisinteressen eingegangen wird (z.B. Herausforderungen/Anforderungen und Potentiale bei den Kooperationspartner). Die Zentrierung der Fragestellungen auf die Studiengangskonzeption bzw. die Methodik und Didaktik der Hochschullehre bedingen gleichzeitig die Reflexion der eigenen Rolle innerhalb der Hochschulverwaltung und der Lehre der Befragten. Forschung ist in diesem Fall gleichzeitig Reflexionsanlass, was bei der Analyse zu berücksichtigen ist (vgl. Meyer \& Müller 2014; Müller 2015). 
Dittmann, Kreutz \& Meyer 2014). Auch eine Differenzierung anhand der auf die zeitliche Organisation bezogenen Studiengangsbezeichnung des jeweiligen Anbieters (d. h. berufsbegleitend, berufsintegrierend oder in Teilzeit) ist problematisch (vgl. dazu ausführlich Dittmann \& Gronewold 2015). Es finden sich bei den räumlichen, zeitlichen und finanziellen Faktoren unterschiedlichste Definitionen in Bezug auf die als berufsbegleitend gekennzeichneten MINT-Studiengänge: Studienangebote mit einem hohen Anteil an Präsenzphasen (z.B. jedes zweite Wochenende) werden als Fernstudium ausgewiesen, Studiengänge mit einem geringen Anteil an Präsenzzeit (nur eine bestimmte Stundenanzahl) werden hingegen als Präsenzstudiengang deklariert.

Für den MINT-Bereich können empirisch somit zum einen eine marginale Präsenz von Universitäten im Feld berufsbegleitender Studiengänge festgestellt werden und folglich eine deutliche Überlegenheit von privaten und staatlichen Fachhochschulen. Zum anderen überwiegen die ingenieurswissenschaftlichen im Gegensatz zu den naturwissenschaftlichen Studiengängen. Darüber hinaus wird die oben genannte Intransparenz in Bezug auf die Organisationsform zusätzlich verschärft, da die Bezeichnung der Studiengänge keiner ausgewiesenen Systematik folgt.

\section{Herausforderungen für Lehrende und Koordinatoren}

Im Rahmen der Analyse o. g. sechszehn qualitativen Interviews konnten für Lehrende und Koordinatoren der berufsbegleitenden MINT-Studiengänge verschiedene Herausforderungen identifiziert werden. Diese resultieren einerseits aus den eben beschriebenen Strukturmerkmalen, andererseits aus einem nicht klar definierten Selbstverständnis innerhalb der Programmatiken der berufsbegleitenden Studiengänge.

Lehrende sind in mehrfacher Hinsicht vor Herausforderungen gestellt: Die Studierenden verfügen aufgrund einschlägiger Berufs- oder Aufstiegsfortbildungen über eine differenzierte Vorbildung sowie Erfahrungswissen, welches sie im Rahmen der Lehrveranstaltungen einbringen. Dieser enge Bezug der Studieninhalte zu der beruflichen Praxis, ist für diese Zielgruppe besonders wichtig, da für sie die direkte Anwendung des neuen Wissens im Vordergrund steht (vgl. dazu auch Wolter, Banscherus, Kamm, Otto \& Spexard 2014). Die Lehrenden sind gefordert dieses Erfahrungswissen in die Lehr-Lern-Praxis einzubinden, d. h. über die Verknüpfung von Theorie- und Erfahrungswissen die o. g. reflexive Handlungsfähigkeit zu fördern. Dabei gilt es den Praxisbezug im Denken der Studierende zu modulieren, denn das Erfahrungswissen und die Vorbildung liegen in unterschiedlichen Graden vor: 
Also natürlich können die Abiturienten besser Mathematik und teilweise Physik. Davon gibt es aber eben andere, die sagen `Ja, in der Praxis... ah, ja, da machen wir das so und so und so, weil... . (...). Und da kommt dann oft sehr gut ein Ergebnis heraus. Wir erleben das auch im Praktikum, gerade dann, wenn die irgendwas aufbauen müssen, natürlich die Praktiker, die schon länger im Beruf sind, da viel fixer sind und sowas dann durchführen, als wie diejenigen, die da natürlich Neuland betreten.(Auszug Interview Dozent)

Die Erfahrungen der beruflich qualifiziert Studierenden stellen sich also als Wissensquelle dar (vgl. dazu auch Dick 2010, 17), die von den Lehrenden genutzt werden kann, um den Wissenstransfer in die Praxis zu ermöglichen. Des Weiteren sind die Lehrenden gefordert den hochschulischen Lernmodus im Sinne eines systematischen Lernens zu vermitteln und bei den Studierenden das sich Einlassen auf das theoretische Wissen zu fördern. Es gilt eine Schärfung der Wissens- und Fähigkeitsorientierung z.B. in Bezug auf das wissenschaftliche Arbeiten zu forcieren:

Die haben halt gelernt Kabel zu ziehen und wenn da Programmierung steht, dann wollen die sofort etwas tippen. Die sind halt sehr fertigkeitsorientiert. Diese Fähigkeitsorientierung muss man dann ein bisschen nachschleifen. Das kommt im Laufe des Studiums, dass die überhaupt ein Buch in die Hand nehmen. Also das ist im Prinzip noch eine der größten Herausforderungen, die prompt zum Lesen zu bringen. (Auszug Interview Dozent)

Es wird deutlich, dass die Verzahnung theoretischen Wissens und beruflichen Erfahrungswissens in berufsbegleitenden Studienmodellen zu einer systematischen, theoriegeleiteten Weiterentwicklung des beruflichen Wissensbestandes der beruflich Qualifizierten führen kann. Lehrende begreifen dies sowohl als neuartige als auch als wesentliche Anforderung in ihrer beruflichen Tätigkeit: Sie sind gefordert das bestehende berufspraktische Wissen mit wissenschaftlich-theoretischen Kompetenzen zu vermitteln. Dieser Einbezug des Erfahrungswissens sowie die reflexive theoriebasierte Bearbeitung von Praxisproblemen, zeigen dass das berufsbegleitende Studium faktisch die Möglichkeit einer Erweiterung der beruflichen Handlungskompetenz bietet.

Eine weitere zentrale Herausforderung für Lehrende ist das Zeitmanagement. Die Bedürfnisse von beruflich qualifiziert Studierenden sind durch die parallele Berufstätigkeit anders gelagert. Beispielsweise besteht die Erwartung, dass auch am Wochenende Fragen beantwortet werden und Übungsaufgaben zur Verfügung gestellt werden:

Es ist so, dass Nachrichten und Anfragen von berufsbegleitenden Studierenden doch etwas häufiger zu unorthodoxen Zeiten reinkommen, (...), weil nine to five, Montag bis Freitag (...) sind die auf ihrem Job und kümmern sich nicht um das Studium (...) die schreiben dann Nachrichten abends um elf oder samstags um $18 \mathrm{Uhr}$, wenn sie zu Hause sind. (Auszug Interview Dozent)

Ähnlich zu der Entgrenzung von Lernort und -zeit (vgl. Arnold 1998) ist im Kontext des berufsbegleitenden Studierens also eine Intensivierung der Bera- 
tung zu verzeichnen, die sich als Entgrenzung der Lernbegleitung äußert. Als weitere Faktoren innerhalb der Herausforderung Zeitmanagement kristallisieren sich die Stofffülle und die methodische und didaktische Aufbereitung heraus. Den Lehrenden ist zwar bewusst, dass durch selbstorganisiertes und selbstgesteuertes Lernen anhand von Fallbeispielen ein tieferes Verständnis für die jeweiligen fachlichen Inhalte entsteht und dadurch auch personale und soziale Kompetenzen entwickelt werden können. Dies zeigt folgendes Zitat:

(...) das reicht bis zur der Art und Weise, wie biete ich den Stoff an. Natülich ist es so, dass es bei den Leuten zu dem tiefsten Verständnis führt und am meisten hängen bleibt, wenn sie sich möglichst viel selbst erschließen anhand von Fallbeispielen, Übungsaufgaben, fallbasiertem Lernen. (Auszug Interview Dozent)

Jedoch sehen sie sich gleichzeitig in dem Dilemma für fallbasiertes Lernen keine Zeit zu haben und zwar aufgrund des umfangreichen Lehrstoffs, der vermittelt werden soll. Stattdessen greifen sie auf Vortrag und Präsentation im Sinne des klassischen Frontalunterrichts zurück.

Bei den berufsbegleitenden Studierenden bin ich dann immer sofort in dem Dilemma, dass ich sehr genau weiß, wie stark eingeschränkt deren Zeitbudget für das Studium ist und ich mir einfach auch überlegen muss, ob ich die jetzt da mit drei Stunden Recherche und selber Knobeln belaste oder ob ich sage „Eine Stunde reicht eigentlich auch“. (Auszug Interview Dozent)

Hier deutet sich ein Wahrnehmungsproblem in Bezug auf das spezifische Angebot des berufsbegleitenden Studierens an: Offenbar wird dieses Bildungsformat als Pendant zu einem grundständigen Studium begriffen und nicht als wissenschaftliche Weiterbildung. Zudem wird durch den Rückgriff auf lehrerzentrierte Formen des Unterrichts die o. g. Möglichkeit der Förderung und Weiterentwicklung reflexiver Handlungsfähigkeit konterkariert. Ebenso fehlt bei dieser Unterrichtsform der interaktive Einbezug des Erfahrungswissens der Studierenden, so dass die Gefahr besteht, dass eine reflexive theoriebasierte Bearbeitung von Praxisproblemen (vgl. Dittmann \& Gronewold 2015, 166) ausbleibt.

Zusammenfassend lassen sich aus der Empirie für die Lehrenden in berufsbegleitenden MINT-Studiengängen der hochschulische Lernmodus, die Einbeziehung des Erfahrungswissen und der Vorbildung sowie das Zeitmanagement als erste wichtige Herausforderungen beschreiben.

Auch die Studiengangskoordinatoren sind mit Herausforderungen konfrontiert, die sich aus diesem spezifischen Bildungsformat ergeben. Beispielsweise ist die Organisation der Räumlichkeiten als Herausforderung zu nennen: Dies äußert sich insbesondere in dem Bereitstellen und dem Herrichten der Labore sowie in der Abstimmung mit externen Laborleitern.

Naja, da ist die Herausforderung eben, die Labore für die Samstage so zu präparieren, dass sie samstags zur Verfügung stehen. Weil montags bis freitags sind andere Veranstaltungen gewesen. Wir mussten dann eben mit den Laborinhabern uns abstimmen, 
weil an den Samstagen wenn Laborübungen sind, nicht eben unbedingt der Vertreter, der hier Laborinhaber ist, dann samstags die Sachen macht, sondern ein Fremder samstags dann in sein Labor geht. (Auszug Interview Koordinator)

Als weitere Herausforderung für Koordinatoren ist die betriebliche Freistellung zu nennen: Bei der Gewinnung potentiell Studierender kann nicht per se auf die Unterstützung in Bezug auf das berufsbegleitende Studium seitens der Betriebe gesetzt werden kann. Folgendes Zitat eines Studiengangskoordinators, der von einer Informationsveranstaltung berichtet, verdeutlicht diese Schwierigkeit:

Und, wir nehmen mal an, wenn da von der Größenordnung 15 Studieninteressierte sind und ich habe beim letzten Mal gefragt: Bei wem käme denn so ein Studiengang in Frage? Dann haben sich von den 15, vielleicht noch drei bis vier gemeldet und wenn sie dann fragen: Wer glaubt denn, dass er das mit seiner Firma hinkriegt? Da hat sich dann nur noch einer gemeldet. (Auszug Interview Koordinator)

Diese mangelnde Kooperationsbereitschaft der Betriebe ist vor dem Hintergrund des häufig beschworenen Arbeitskräftebedarfs - insbesondere in den MINT-Branchen - sowie des Qualifikationsstrukturwandels (vgl. exemplarisch Severing \& Teichler 2013) besonders irritierend: Seitens der Wirtschaft wird prognostiziert, dass sich die Arbeitskräfteengpässe weiter verschärfen könnten, weil die wirtschaftliche und technologische Leistungsfähigkeit von den innovationsstarken MINT-Branchen bestimmt wird (vgl. exempl. BMBF 2012, 26f.) und jene „,beruflichen Tätigkeitsbereiche wachsen, für die im Prinzip sowohl eine beruflich-betriebliche Ausbildung als auch ein Studium“ (vgl. Severing \& Teichler 2013, 11) notwendig werden. Dennoch zeigt die vorliegende Analyse, dass sich dies nicht unmittelbar auf die Zusammenarbeit von Hochschule und Unternehmen auswirkt.

Im Kontext der betrieblichen Freistellung kommen Studiengangskoordinatoren zudem immer wieder in Situationen, in denen sie damit konfrontiert sind Unterstützung bei der Verbindung von Arbeiten und Lernen zu leisten. Nicht selten sind sie gefordert berufliche Tätigkeiten, wie das Unternehmen von Dienstreisen, mit lernbezogenen Tätigkeiten, wie die Organisation von Klausuren und Prüfungen, zu koordinieren.

Es gibt auch immer wieder Situationen, wo mich dann die Studierenden anrufen und mir im Vorfeld schon sagen „Sie wissen doch, ich bin dann und dann auf Dienstreise zwei, drei Wochen, legen sie da bitte keine Klausur hin“. (Auszug Interview Koordinator)

In dieser Herausforderung drückt sich der von Bülow-Schramm (2014) formulierte Apell aus, dass die Hochschulen der veränderten studentischen Lebenswirklichkeit aufgrund der zunehmenden Heterogenität in der Zusammensetzung der Studierenden gerecht werden müssen. Studienangebote und -organisation gilt es entsprechend anzupassen (vgl. ebd.). Dies ist insbesondere mit Blick auf die Zielgruppe der beruflich Qualifizierten wesentlich. 
Darüber hinaus hat sich die Art und Weise der Studienbetreuung gewandelt. Die Studiengangskoordinatoren haben neben der Organisation und Vorbereitung der Prüfungen auch die Aufgabe Testklausuren zu organisieren und Unterstützung in kritischen Fächern zu vermitteln. Diese Art der persönlichen und unterstützenden Betreuung sehen sie als eine ihrer wichtigsten Aufgaben. Dabei sind sich die Koordinierenden den Bedürfnissen und der besonderen Stellung dieser neuen Zielgruppe bewusst.

Bei technischen Fächern gibt es immer ein paar kritische Fächer. (...). In diesem Studiengang (...) haben wir grade wegen des hohen Anteils beruflich Qualifizierten doch erhebliche Probleme, sie alle durch die Prüfung zu kriegen. Wir geben uns da sehr viel Mühe. Das geht über organisierte Testklausuren, persönliches Coaching, Prüfungsvorbereitung. Und die Prüfung ist nicht leichter als für andere auch. Aber die Vorbereitung sehen wir doch sehr persönlich und auch als Teil unserer Aufgabe der Studienbetreuung. (Auszug Interview Koordinator)

Insgesamt zeigt die Auswertung, dass die koordinierenden Tätigkeiten im Rahmen von berufsbegleitenden Studiengängen an Komplexität gewonnen haben: Die Planung und das Organisieren von Semester- und Prüfungszeiten, die Strukturierung und Verbindung von Arbeits- und Lernzeiten und Räumlichkeiten sowie die Betreuung der Studierenden und die Gewinnung potentiell Studierender, sind in Abstimmung mit externen Partnern (Betriebe, Unternehmen aber auch Labore, die für praktische Übungen benötigt werden) zu leisten. In diesen Abstimmungsprozessen sind gegenseitige Abhängigkeiten zu konstatieren, die die Kooperation mit den jeweiligen Partnern erschweren und neue Anforderungen an die Zusammenarbeit stellen.

\section{Anforderungen und Entwicklungspotentiale für Kooperationspartner}

Für die Hochschulen sind unterschiedliche Kooperationspartner von Bedeutung. Zum einen stellen Kammern (IHK und HWK) sowie Bildungsakademien Kooperationspartner dar. Sie arbeiten vor allem in Bezug auf das Thema Anrechnung von bereits erbrachten Leistungen zusammen. Konkret geht es darum, das berufsbegleitende Studium durch die systematischen Anrechnungen von Leistungen aus einschlägigen Aufstiegsfortbildungen, z.B. Meister oder Techniker, in finanzieller Hinsicht zu entlasten und in zeitlicher Weise zu verkürzen. Ein Koordinator berichtet dazu folgendes:

Weil es tatsächlich so ist, dass wir aus der Technikerausbildung Anrechnungen für unser Studium vornehmen können, in den Grundlagen. Das heißt, Studierende, die mit der Technikerausbildung zu uns kommen, denen können wir gewisse Leistungen schon anerkennen. (Auszug Interview Koordinator) 
Auch Loroff und Freitag (2011) kommen zu dem Ergebnis, dass diese pauschalen oder spezifischen Anrechnungen z. T. bereits in der Konzeptionsphase von beruflichen Fortbildungsangeboten berücksichtigt werden. Inhalte und Lernziele werden so konzipiert und ausgewählt, das sie fachlich an das Profil des jeweiligen Studiengangs anschlussfähig sind (vgl. ebd.). Damit leistet die Kooperation zwischen Kammern bzw. Bildungsakademien und Hochschulen einen Beitrag, um den Weg von der beruflichen in die hochschulische Bildung zu erleichtern und trägt insofern zu der Durchlässigkeit zwischen den Bildungsbereichen bei.

Zum anderen sind Personalabteilungen bzw. die Personalentwicklung von Unternehmen weitere Partner im Rahmen der Kooperationsprozesse. Allerdings wird die Kooperationsbereitschaft der Betriebe als mangelhaft und wenig befriedigend beschrieben. So erläutert ein Dozent in einem Interview wie folgt:

Nur in ganz wenigen Ausnahmefällen funktioniert das, was wir uns eigentlich vorgestellt hatten, nämlich eine Personalentwicklungsmaßnahme. Das eine Firma eine Mitarbeiterin oder einen Mitarbeiter irgendwie identifiziert, denen man einen beruflichen Aufstieg zutraut und den man begleiten möchte. (Auszug Interview Dozent)

Betriebe bzw. Personalentwicklungsabteilungen sehen es offenbar nicht als ihre Aufgabe an beruflich Qualifizierte für ein berufsbegleitendes Studium zu gewinnen und damit auch die Möglichkeiten zur Weiterentwicklung der Beschäftigten zu gestalten und zu optimieren.

Als weitere Kooperationspartner sind die Gewerkschaften zu nennen, die aus Sicht der Hochschulen bisher allerdings eine eher marginale Rolle spielen. Zwar haben Gewerkschaften die Weiterentwicklung ihrer Mitglieder auch in Bezug auf die wissenschaftliche Weiterbildung als zentrales Ziel formuliert (vgl. DBG 2012), jedoch ist ihr Interesse an dem Format berufsbegleitendes Studium und die Beteiligung im Sinne des Aufzeigens potentieller Entwicklungswege nur gering ausgeprägt.

Da hat ein Jugendvertreter angerufen. Die machen innerhalb der Ausbildungsabteilung eine Informationsrunde, um ihren eigenen Kolleginnen und Kollegen quasi zu sagen, also wenn ihr ausgelernt habt, was könnt ihr dann weiter machen. Das ist auch neu. Das hatte ich auch noch nicht. Da bin ich mal sehr neugierig. (Auszug Interview Dozent)

In Bezug auf das berufsbegleitende Studium stellen die Gewerkschaften für die Hochschulen ein neues und bisher unbekanntes Kooperationsfeld dar, dem sie mit Interesse begegnen. Hochschulen sind darauf angewiesen mit den Sozialpartnern zusammenzuarbeiten, um die Durchlässigkeitsthematik, die beispielswiese auch im hochschulpolitischen Programm des DGB (vgl. ebd.) vertreten ist, nachhaltig zu gestalten. Da Gewerkschaften als ein zentraler Akteur an der Gestaltung der Berufsbildungspolitik beteiligt sind, ist das 
berufsbegleitende Studium in dieser Hinsicht ein Thema, das es noch stärker $\mathrm{zu}$ fokussieren gilt.

\section{Fazit - Veränderung und Weiterentwicklung der Bildungsorganisation und -struktur}

Auf Basis der hier beschriebenen und analysierten empirischen Daten lässt sich zunächst festhalten, dass es im Bereich der berufsbegleitenden Studienformate häufig an Ressourcen (didaktisch und methodisch vgl. Kap. 4) und an adäquaten und verbindlich organisatorischen Strukturen (vgl. Kap. 3) zu mangeln scheint. Das lässt darauf schließen, dass diese Angebote von den Anbietern selbst oft noch als Randerscheinungen des Studienangebotes betrachtet werden (vgl. dazu auch Minks et al. 2011, 99). Aus den dargestellten Herausforderungen der Koordinatoren und Lehrenden sowie aus der Bedeutung der Kooperationspartner können mit Blick auf die oben beschriebenen Strukturmerkmale der berufsbegleitenden MINT-Studiengänge Thesen abgeleitet werden, die eine kontinuierliche Veränderung der Bildungsorganisation und -struktur auf den verschiedenen Ebenen (Mikro, Meso, Makro) erforderlich machen.

Für die (Mikro-)Ebene der Lehrenden ist die Entwicklung und Implementierung von didaktischen Modellen für berufliche Qualifizierte notwendig.

Für das nachhaltige Bestehen berufsbegleitender Studienformate stellt sich die Entwicklung und Implementierung von didaktischen Modellen für die beruflich qualifiziert Studierenden als zentraler Faktor dar: In diesen Modellen ist die explizite Verknüpfung von Theorie und Praxis anzustreben, um beispielsweise die benannte Stofffülle zu bewältigen. Vor allem gilt es das Erfahrungswissen der Studierenden als Gegenstand der LehrLerninteraktion zu betrachten (vgl. Meyer \& Kreutz 2015, 172) und dazu sinnvolle Lehr-Lernformen zu entwickeln. Ebenso könnte es dadurch gelingen die reflexive Handlungsfähigkeit der Studierenden weiterzuentwickeln und die oben geforderte reflexive theoriebasierte Bearbeitung von Praxisproblemen, könnte dadurch teifgreifender erfolgen.

Für die (Mikro-)Ebene der Koordinatoren ist eine Flexibilisierung des Studiums mit Bezug auf die spezifischen Bedürfnisse der Zielgruppe anzusteuern.

Dadurch könnte sowohl eine gezielte Verbindung von Arbeiten und Lernen als auch ein ausgewogenes Gleichgewicht zwischen diesen Bereichen erreicht werden. Beispielsweise könnten Studiengangskoordinatoren und Kooperationspartner im Rahmen der Akquise von potentiellen Studierenden eine flexiblere und individuellere Gestaltung des Verhältnisses von Arbeiten und Lernen befürworten. Eine Orientierung würden beispielsweise Organisa- 
tionsformen geben, die Arbeiten und Lernen gezielt miteinander verbinden (vgl. exempl. Dehnbostel 2007); wobei die Modelle arbeitsbezogenen Lernens in Bezug auf ihre „Tauglichkeit“ für ein berufsbegleitendes Studium geprüft werden müssten.

Für die (Meso-)Ebene der Kooperationspartner ist zu konstatieren, dass berufliche und hochschulische Bildung als ,Gesamtkonzept “ einer erweiterten bzw. beruflichen Handlungskompetenz zu begreifen ist.

Zurzeit erschwert allerdings die o. g. mangelnde Intransparenz bzgl. der Organisationsform eines jeweiligen Studiengangs den Personalentwicklern (Kooperationspartner) das berufsbegleitende Studium als Personalentwicklungsmaßnahme zu etablieren und geeignete Beschäftigte dafür zu finden. Die Hochschulrektorenkonferenz (HRK) sprach sich bereits 1993 explizit dafür aus, eine Differenzierung zwischen grundständigem Studium, wissenschaftlichem Aufbau- und Vertiefungsstudium sowie einem berufsorientiertem Weiterbildungsstudium vorzunehmen (vgl. HRK 1993). Die Aufgabe der berufsbezogenen wissenschaftlichen Weiterbildung an Hochschulen ist somit nicht neu. Draus folgt, dass auf der (Makro-)Ebene der Institution Hochschule eine Erweiterung des Selbstverständnisses Bedeutung erhält, um die angestrebte Durchlässigkeit im Bildungssystem tatsächlich gewährleisten zu können. Dazu gehört beispielsweise auch eine klare Definition hinsichtlich der Zielsetzung und der Ausdifferenzierung der Programmatik in Bezug auf den Bereich der wissenschaftlichen Weiterbildung.

\section{Literatur}

Arbeitsgemeinschaft Berufsbildungsforschungsnetz (AG BFN) (2014). Verzahnung beruflicher und akademischer Bildung - duale Studiengänge in Theorie und Praxis. Tagungsprogramm. Online unter: http://www.imovegermany.de /cps/rde/ xbcr/SID-1F9D14E2-016AD36F/kibb/AGBFN_Programm_Duales_Studium_ 141106.pdf (31.03.2016).

Arnold, R. (1998). Kompetenzentwicklung und Organisationslernen. In N. Vogel (Hrsg.), Organisation und Entwicklung der Weiterbildung (S. 86-110). Bad Heilbrunn: Klinkhardt.

Bauer, H. G., Böhle, F., Munz, C., Preiffer, S. \& Woicke P. (2006). Hightech-Gespür. Erfahrungsgeleitetes Arbeiten und Lernen in hochtechnisierten Arbeitsbereichen. Aktualisierte und ergänzte Fassung. Bielefeld: W. Bertelsmann Verlag.

Bundesministerium für Bildung und Forschung (BMBF) (Hrsg.). (2012). Perspektive MINT. Wegweiser für MINT-Förderung und Karrieren in Mathematik, Informatik, Naturwissenschaften und Technik. Online unter: http://www.bmbf.de/pub/ perspektive_mint.pdf (02.06.2015). 
Bülow-Schramm, M. (2014). Durchlässigkeit als Zielmarke für Übergänge im Hochschulsystem? Zur Ambivalenz von Heterogenität und Homogenität in der Hochschule. In U. Banscherus, M. Bülow-Schramm, K. Himpele, S. Stack \& S. Winter (Hrsg.), Übergänge im Spannungsfeld von Expansion und Exklusion. Eine Analyse der Schnittstellen im deutschen Hochschulsystem (S. 269-286). Bielefeld: W. Berteslmann Verlag.

Dehnbostel, P. (2007). Lernen im Prozess der Arbeit. Münster: Waxmann.

Deutscher Gewerkschaftsbund (2012). Für eine demokratische und soziale Hochschule. Das hochschulpolitische Programm des Deutschen Gewerkschaftsbundes. Berlin.

Dick, M. (2010). Ungenutzte Potenziale: Weiterbildung an Hochschulen als Transformation zwischen Wissenschaft und Praxis. Zeitschrift für Soziologie der Erziehung und Sozialisation, 1, 13-25.

Dittmann, C., Kreutz, M. \& Meyer, R. (2014). Gefilterte Fahrstuhleffekte?! Herausforderungen des berufsbegleitenden Studiums in der Perspektive berufserfahrener Lernender.bwp@Berufs-und Wirtschaftspädagogik-online, 26, 1-26. Online: http://www.bwpat.de/ausgabe26/dittmann_etal bwpat26.pdf (29.09.2015).

Dittmann, C. \& Gronewold, J. K. (2015). Berufsbegleitende Studienkonzepte im MINT-Bereich - Die Verbindung beruflichen und akademischen Wissens als zentrale Herausforderung der Studiengangskonzeption. In U. Elsholz (Hrsg.), Beruflich Qualifizierte im Studium. Analyse und Konzepte zum Dritten Bildungsweg (S. 165-177). Bielefeld: W. Berteslmann Verlag.

Freitag, W. K. \& Loroff, C. (2011). Anrechnung beruflicher Kompetenzen auf Hochschulstudiengänge (ANKOM) - Einführung und Überblick. In W. K. Freitag, E. A. Hartmann, C. Loroff, I. Stamm-Riemer, D. Völk \& R. Buhr (Hrsg.): Gestaltungsfeld Anrechnung. Hochschulische und berufliche Bildung im Wandel (S. 917). Münster: Waxmann.

Gläser, J. \& Laudel, G. (2006). Experteninterviews und qualitative Inhaltsanalyse. Wiesbaden: VS Springer.

Heublein, U., Richter, J., Schmelzer, R. \& Sommer, D. (2012). Die Entwicklung der Schwund und Studienabbruchquoten an den deutschen Hochschulen Statistische Berechnungen auf der Basis des Absolventenjahrgangs 2010. Online: http:// www.dzhw.eu/ pdf/pub_fh/fh-201203.pdf. (19.10.2015).

Hochschulrektorenkonferenz [HRK] (1993). Die wissenschaftliche Weiterbildung an den Hochschulen. Entschließung des 170. Plenums vom 12. Juli 1993. Online: http://www.hrk.de/positionen/beschluesse-nach-thema/convention/die-wissenschaftliche-weiterbildung-an-den-hochschulen/ (17.10.2015).

Mayring, P. (2002). Einführung in die qualitative Sozialforschung. Weinheim und Basel: Beltz.

Meyer, R. \& Kreutz, M. (2015). Die Verzahnung beruflichen und wissenschaftlichen Wissens - Perspektiven für Forschung und Praxis am Beispiel des Dritten Bildungsweges. In A. Dietzen, J. J. W. Powell, A. Bahl \& L. Lassnigg (Hrsg.), Soziale Inwertsetzung von Wissen, Erfahrung und Kompetenz in der Berufsbildung (S. 160-176). Weinheim und Basel: Beltz. 
Meyer, R. \& Müller, J. K. (2014). Forschung als Reflexionsanlass und Beitrag zur Organisationsentwicklung. In C.H. Antoni, P. Friedrich, A. Haunschild, M. Josten \& R. Meyer (Hrsg.), Work-Learn-Life-Balance in der Wissensarbeit. Herausforderungen, Erfolgsfaktoren und Gestaltungshilfen für die betriebliche Praxis (S. 179-195). Wiesbaden: VS Springer.

Minks, K.-H., Netz, N. \& Völk, D. (2011). Berufsbegleitende und duale Studienangebote in Deutschland: Status quo und Perspektiven. Online: http://www.dzhw.eu /pdf/pub_fh/fh-201111.pdf(31.03.2016).

Müller, J. K. (2015): Reflexion als Voraussetzung für Kompetenz- und Organisationsentwicklung in der wissensintensiven Arbeit. Detmold: Eusl-Verlagsgesellschaft.

Pätzold, G. (2011). Berufliche und hochschulische Bildung im Wandel - Statt institutioneller Differenzierung Kooperation, Vernetzung und Durchlässigkeit. Zeitschrift für Berufs- und Wirtschaftspädagogik, 107(4), 481-498.

Rein, V. \& Kolter, C. (2013). Durchlässigkeitsfördernde Aspekte der Gestaltung von Bildungsgängen an der Schnittstelle beruflicher und hochschulischer Bildung im Kontext der Kompetenz- und Lernergebnisorientierung. Projektbeschreibung. Online: https://www2.bibb.de/bibbtools/tools/fodb/data/documents/pdf/at_33304 .pdf (31.03.2016).

Severing, E. \& Teichler, U. (Hrsg.). (2013). Akademisierung der Berufswelt? Bielefeld: W. Berteslmann Verlag.

Statistisches Bundesamt (Destatis) (Hrsg.) (2013). Bildung und Kultur. Studierende an Hochschulen - Fächersystematik. Wiesbaden.

Vogler-Ludwig, K. \& Düll, N. (2013). Arbeitsmarkt 2030. Eine strategische Vorausschau auf Demografie, Beschäftigung und Bildung in Deutschland. Bielefeld: W. Bertelsmann Verlag.

Wolter, A., Banscherus, U., Kamm, C., Otto, A. \& Spexard, A. (2014). Durchlässigkeit zwischen beruflicher und akademischer Bildung als mehrstufiges Konzept: Bilanz und Perspektiven. Beiträge zur Hochschulforschung, 36(4), 8-39. 


\title{
Entwicklung eines Modells zur Beurteilung der Qualität von berufsbegleitenden Studiengängen
}

\author{
Lena Hillebrecht
}

\section{Einleitung}

Die Bedeutung berufsbegleitender Bildungsangebote auf akademischem Niveau hat in den letzten Jahren zugenommen (vgl. Wolter \& Geffers 2013), jedoch nicht in dem Ausmaß, das mittels bildungspolitischer Initiativen wie der Hochschulöffnung für beruflich Qualifizierte (vgl. KMK 2009) beabsichtigt wurde. Es sollte die Studierendenquote unter Berufstätigen gesteigert werden, indem die Zahl der Studienberechtigten deutlich erhöht wurde (vgl. Wolter, Banscherus, Kamm \& Otto 2014). Zudem ist festzustellen, dass die Inanspruchnahme von Angeboten des Lebenslangen Lernens an Hochschulen in Deutschland im internationalen Vergleich eher gering ausfällt (vgl. Wolter et al. 2014). Ein möglicher Erklärungsansatz für die geringe Inanspruchnahme bestehender Studienangebote ist die Vermutung, dass die derzeit vorhandenen Studienmodelle aus Sicht der Studierenden nicht über eine ausreichende Passgenauigkeit und Qualität verfügen und daher für die Zielgruppe nicht ausreichend attraktiv sind (vgl. Wolter et al. 2014).

Gegenwärtig ist jedoch kein Instrument vorzufinden, das sich zur Beurteilung der Qualität dieser Studienmodelle einsetzen ließe, weshalb dessen Entwicklung und empirische Überprüfung erforderlich ist (vgl. Minks, Netz \& Völk 2011; Wolter et al. 2014). Ziel des in diesem Beitrag beschriebenen Vorhabens ist es daher, Studiengangsmerkmale zu identifizieren, die aus der Sicht der Studierenden für die Beurteilung von Qualität in berufsbegleitenden Studiengängen relevant sind. Diese Merkmale werden in einem Modell abgebildet, das mit einer quantitativen Studie überprüft wird. Für die Überprüfung wurde zunächst ein Fragebogen entwickelt, der eine Operationalisierung der Modellparameter darstellt. Anschließend wurde das so entwickelte Messinstrument im Rahmen einer quantitativen Erhebung pilotiert, um es anschließend in einer größeren Stichprobe einzusetzen. Im vorliegenden Beitrag liegt der Schwerpunkt auf der Darstellung der Ergebnisse der Modellpilotierung. 


\section{Forschungsstand}

Ausgangspunkt der Untersuchung von berufsbegleitenden Studiengängen bildet bei dem hier beschriebenen Vorhaben das „Rahmenmodell zur Wirkungsanalyse des Hochschulunterrichts“ von Helmke und Schrader (2006). Hierbei wird davon ausgegangen, dass die Effekte des Studiums, wie der Aufbau von Fachwissen oder Expertise, dadurch zustande kommen, dass die Lernenden auf Grundlage ihrer individuellen Studienbedingungen und Lernvoraussetzungen das Lehrangebot in einer individuellen Weise nutzen. Die Lehr-Lernsituation ist zudem eingebettet in den fachlichen, sozialen und ökologischen Kontext sowie die soziokulturellen Rahmenbedingungen (vgl. Helmke \& Schrader 2006).

Im vorliegenden Beitrag werden berufsbegleitende Studiengänge in den Blick genommen. Darunter werden Studiengänge verstanden, die von Berufstätigen absolviert werden und die speziell für diese Zielgruppe konzipiert wurden (vgl. Bargel \& Bargel 2014). Daher spielt auch die Zielgruppe und deren Belange eine wichtige Rolle bei der Beschäftigung mit diesen Studiengängen. Für die Beschreibung der Zielgruppe sind vor allem die Begriffe der nicht-traditionell Studierenden und der beruflich qualifizierten Studierenden heranzuziehen. Mit der ersten Bezeichnung sind Studierende gemeint, ,die nicht auf dem geraden Weg bzw. in der vorherrschenden zeitlichen Sequenz und Dauer zur Hochschule gekommen sind; nicht die regulären schulischen Voraussetzungen für den Zugang erfüllen; und solche, die nicht in der üblichen Form des Vollzeit- und Präsenzstudiums studieren (sondern als Teilzeit-, Abend- und Fernstudierende)“ (vgl. Teichler \& Wolter 2004).

Unter dem Begriff der beruflich Qualifizierten werden Personen gefasst, die eine berufliche Ausbildung erworben haben und zusätzlich eine Hochschulzugangsberechtigung besitzen oder Personen, die über den dritten Bildungsweg zum Studium gelangt sind (vgl. Autorengruppe Bildungsberichterstattung 2014). Es kann also davon ausgegangen werden, dass in berufsbegleitenden Studiengängen anteilig mehr Studierende bereits eine berufliche Vorbildung haben als in Vollzeit-Studiengängen. Folglich lässt sich festhalten, dass sich die Studierenden in berufsbegleitenden Studiengängen vor allem hinsichtlich ihrer Eingebundenheit durch berufliche Verpflichtungen und durch das häufige Vorhandensein beruflicher Vorqualifikationen auszeichnen. Es ist also davon auszugehen, dass sowohl Studierende mit einer schulischen, als auch Studierende mit einer beruflichen Hochschulzugangsberechtigung ein solches Studium aufnehmen (vgl. Wolter $\&$ Geffers 2013). Für die berufsbegleitend Studierenden ist folglich anzunehmen, dass es sich hierbei um eine Studierendengruppe mit stark ausgeprägter Heterogenität in Bezug auf die individuellen Lernvoraussetzungen 
handelt. Es kann angenommen werden, dass einige Studierende bereits über einschlägiges Vorwissen verfügen, das sie in einer beruflichen Vorbildung erworben haben. Defizite bzgl. der studienrelevanten Vorkenntnisse sind im Bereich Mathematik und beim wissenschaftlichen Arbeiten zu vermuten, was v. a. für Studierende gilt, die nicht über eine schulische Hochschulzugangsberechtigung verfügen (vgl. Wolter \& Geffers 2013).

Bezüglich der soziodemografischen Merkmale ist zu erwarten, dass die berufsbegleitend Studierenden im Mittel älter sind als diejenigen in VollzeitStudiengängen und dass sie häufiger bereits eine eigene Familie gegründet haben, was z.B. mit eingeschränkter örtlicher Mobilität und geringeren zeitlichen Ressourcen einhergehen kann, die zusätzlich durch die Berufstätigkeit eingeschränkt werden (vgl. Wolter \& Geffers 2013; Zinn 2012).

Bei berufsbegleitenden Studiengängen ist die Studieneingangsphase von besonderer Bedeutung. Hier sollten sowohl fachliche als auch strukturelle Beratungsangebote vorhanden sein (vgl. Minks et al. 2011), da vielfach von einer großen Unsicherheit bei der Entscheidung für ein Studium auszugehen ist (vgl. Arnold, Wetzel \& Dobmann 2014). Für die Entscheidung über die Aufnahme des Studiums ist es außerdem wichtig, dass die Durchlässigkeit zum Studium gewährleistet wird (vgl. Minks et al. 2011). Dies kann über die Schaffung flexibler Zugangswege zum Studium ermöglicht werden, was neben der Anerkennung von Abschlüssen auch die Gewährung von Anrechnungsoptionen für bereits erbrachte Leistungen (vgl. Wolter \& Geffers 2013) beinhalten kann. Außerdem kann die Durchlässigkeit durch Möglichkeiten zum Probestudium (vgl. Wolter 2000) oder Brückenkurse (vgl. Nickel \& Duong 2012) erhöht werden.

Es ist weiterhin davon auszugehen, dass die berufsbegleitend Studierenden nicht nur zu Beginn, sondern auch während des Studiums einen hohen Beratungsbedarf besitzen. Daher sollten für die Qualitätsbeurteilung auch die Beratungsleistungen der Hochschule während des Studiums berücksichtigt werden (vgl. Arnold et al. 2014).

Bei der Organisation des Studiums ist vermutlich eine flexible zeitliche Organisation bedeutsam, die Raum lässt für freie Zeiteinteilung durch die Lernenden (vgl. Schuetze \& Slowey 2000). Die Organisation in Teilzeit sollte so gestaltet sein, dass die Vereinbarkeit von Studium, Beruf und Privatleben gewährleistet wird (vgl. Schuetze \& Slowey 2000), was auch durch den Einbezug von geeigneten E-Learning-Sequenzen unterstützt werden kann (vgl. Schuetze \& Slowey 2000; Zawacki-Richter, von Prümmer \& Stöter 2015).

Der Zusammenhang zwischen Berufstätigkeit und Studienerfolg bzw. Studienzufriedenheit wurde insbesondere im angloamerikanischen Sprachraum untersucht. Riggert, Boyle, Petrosko, Ash und Parkins (2006) geben in ihrem Literature-Review einen Überblick über den Forschungsstand zur Thematik und schlussfolgern, dass die vorliegenden Studien zu uneinheitlichen 
Ergebnissen kommen: Die wöchentliche Arbeitszeit als Indikator für die berufliche Eingebundenheit hat in manchen Studien einen negativen, in anderen einen positiven Einfluss auf den Studienerfolg. Zudem stellen die Autoren heraus, dass kein theoretisches Modell zur Erklärung des Zusammenhangs zwischen Berufstätigkeit und Studienerfolg vorliegt. Anhaltspunkte hierfür lassen sich lediglich punktuell bei Modellen zur Erklärung des Studienabbruchs finden, in denen Art und Umfang der Berufstätigkeit als Einflussfaktor einbezogen werden (vgl. Riggert et al. 2006).

Weitere Erkenntnisse aus dem angloamerikanischen Raum liegen zu den Unterschieden zwischen nicht-traditionell Studierenden und den traditionell Studierenden vor. Donohue und Wong (1997) zeigten, dass bei nicht-traditionell Studierenden eine höhere Zielstrebigkeit im Studium zu beobachten ist. Es bleibt jedoch fraglich, ob Erkenntnisse aus dem angloamerikanischen Raum aufgrund der großen Unterschiede zwischen dem US-amerikanischen und dem deutschen Bildungssystem ohne Weiteres auf deutsche Studiengänge übertragen werden können (vgl. Zinn 2012).

Anknüpfungspunkte für die Untersuchung von berufsbegleitenden Studiengängen können bei Studien gewonnen werden, die Vollzeit-Studiengänge in den Blick nehmen, wie das SMILE-Projekt (vgl. Schiefele, Streblow, Ermgassen \& Moschner 2003) oder die Befragung der BA-Studierenden der FU Berlin (vgl. Thiel, Veit, Blüthmann, Lepa \& Ficzko 2008). Diese Studien liefern wichtige Erkenntnisse zu den Wirkungszusammenhängen zwischen Studienangebot, Studierendenmerkmalen und Studienerfolg im Allgemeinen. Es kann jedoch davon ausgegangen werden, dass bei berufsbegleitenden Studiengängen deren spezifische Besonderheiten zu berücksichtigen sind. Ähnlich gestaltete Studien wie die beiden hier exemplarisch angeführten wurden bisher noch nicht für berufsbegleitende Studiengänge durchgeführt.

Es lässt sich zusammenfassend festhalten, dass es einige Aspekte gibt, die für die Beurteilung von Qualität in berufsbegleitenden Studiengängen relevant erscheinen. Ein Modell, dass die empirische Untersuchung von Qualität ermöglichen könnte, liegt derzeit jedoch nicht vor (vgl. Wolter et al. 2014). 


\section{Fragestellung}

Das Ziel des hier beschriebenen Vorhabens ist es, ein Modell zu entwickeln und emprisch zu überprüfen, dass die Messung von Qualität in berufsbegleitenden Studiengängen ermöglichen soll. Wie bereits erläutert, liegt das Angebots-Nutzungs-Modell von Helmke und Schrader (2006) zugrunde. Das Modell bezieht sich in seiner Ausgestaltung durch die Autoren vordergründig auf einzelne Lehrveranstaltungen. In der hier beschriebenen Studie werden allerdings Studiengänge als Ganzes in den Blick genommen. Daher wird das Modell erweitert und auf der Angebotsseite wird nicht nur das Lehrangebot, sondern auch die strukturellen Studienbedingungen betrachtet. Dementsprechend wird bei der Betrachtung der Nutzung des Studienangebots sowohl die individuelle Nutzung des Lehrangebots, als auch die der organisatorischen Rahmenbedingungen berücksichtigt.

Zur Identifikation von relevanten Modellparametern bei berufsbegleitenden Studiengängen im Unterschied zu Vollzeit-Studiengängen wurde zunächst eine qualitative Studie durchgeführt, mittels derer Daten anhand von leitfadengestützten Experteninterviews mit drei verschiedenen Zielgruppen (Studierende: $n=7$, Hochschulmitarbeitende: $n=4$ und Arbeitgeber: $n=4$ ) erhoben wurden. Die Interviewtranskripte wurden im Zuge der zusammenfassenden Inhaltsanalyse nach Mayring (2010) ausgewertet, die Ergebnisse dieser Auswertung sind in dem nachfolgend beschriebenen Modell zusammengefasst.

Bei dem entwickelten Modell (Abb. 1) ist als abhängige Variable die Zufriedenheit der Studierenden mit den Gegebenheiten ihres Studiengangs gewählt. Dies soll die Beurteilung der Studienqualität durch die fokussierte Zielgruppe repräsentieren. 
Abb. 1: Modell zur Beurteilung von Qualität in berufsbegleitenden Studiengängen

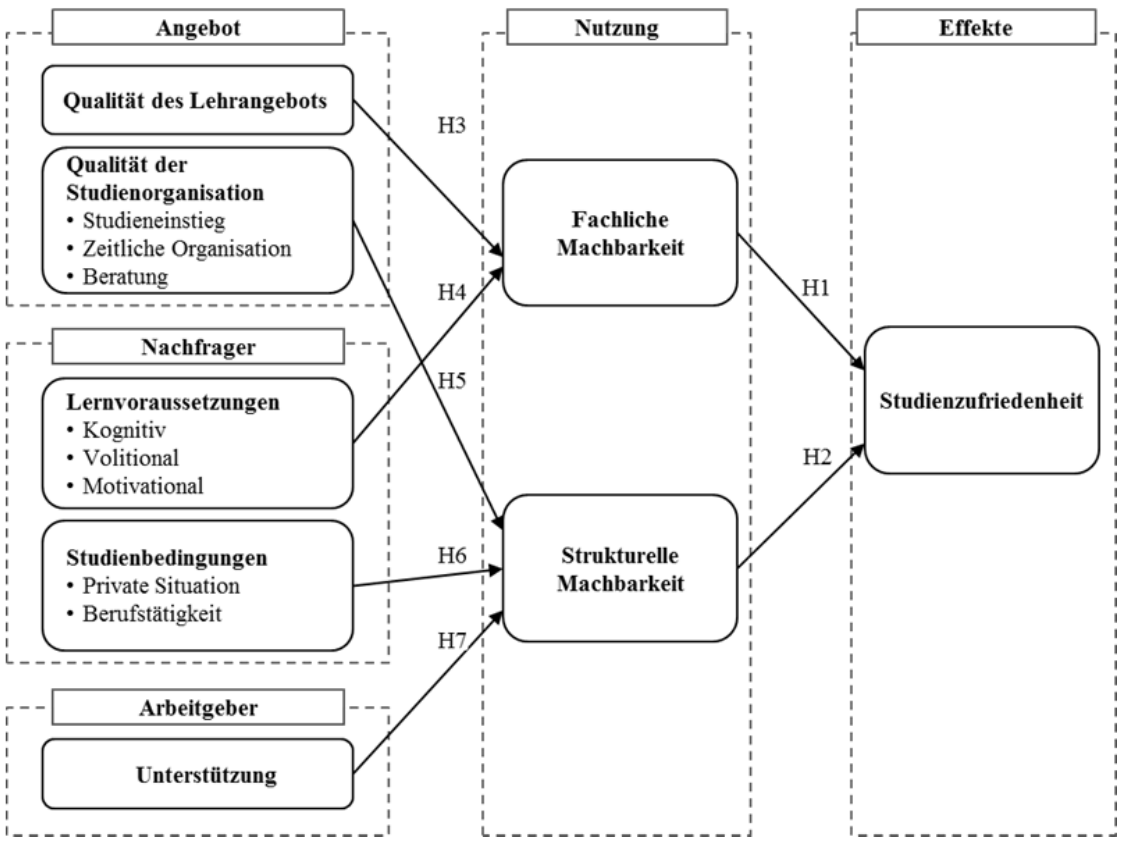

Quelle: eigene Abbildung

Dieses Qualitätsurteil wird im Modell auf zwei Aspekte zurückgeführt: die fachliche und die strukturelle Machbarkeit des Studiums. Gemeint ist, dass in diesen Machbarkeitsdimensionen die Studiengangsmerkmale auf die Studierendenmerkmale treffen und es $\mathrm{zu}$ einem bestimmten Grad an Passung zwischen den Merkmalen kommt, der zu einer fachlichen bzw. strukturellen Machbarkeit des Studiums aus Sicht des Studierenden führt. Demnach sind die Machbarkeitsdimensionen ein Ergebnis aus der Nutzung des Studienangebots auf Grundlage der Studierendencharakteristika. Aus diesem Modell ergeben sich die folgenden Forschungshypothesen.

H1: Ein hohes Maß an fachlicher Machbarkeit führt zu einer hohen Studienzufriedenheit.

H2: Ein hohes Maß an struktureller Machbarkeit führt zu einer hohen Studienzufriedenheit.

Durch diese Hypothesen kommt die Annahme zum Ausdruck, dass im berufsbegleitenden Studium sowohl die fachlichen Aspekte, die die eigentlichen Lehrveranstaltungen betreffen, als auch die organisationalen Rahmen- 
bedingungen einen Einfluss auf die Studienqualität haben. Das Zustandekommen der fachlichen Machbarkeit ist zum einen auf die Qualität des Lehrangebots zurückzuführen. Dieses Angebot wird von den Studierenden auf Grundlage ihrer kognitiven, volitionalen und motivationalen Lernvoraussetzungen individuell in Anspruch genommen. Es werden daher die folgenden Zusammenhänge angenommen:

H3: Je höher die Qualität des Lehrangebots, desto höher ist das Maß an fachlicher Machbarkeit.

H4: Je besser die individuellen Lernvoraussetzungen ausgeprägt sind, desto höher ist das Maß an fachlicher Machbarkeit.

Folglich lässt sich das Zustandekommen der fachlichen Machbarkeit so erläutern, dass die Nutzung des Lehrangebots durch die Studierenden unter Einfluss der individuellen Lernvoraussetzungen $\mathrm{zu}$ einem bestimmten Maß an fachlicher Passung führt.

Für die strukturelle Machbarkeit wird angenommen, dass diese insbesondere in berufsbegleitenden Studiengängen bedeutsam ist. Dies ist darauf zurückzuführen, dass die Studierenden durch ihre Berufstätigkeit und ihre private Situation stärker eingespannt sind und sich die Vereinbarkeit von Studium, Beruf und Privatem vermutlich schwieriger gestalten lässt, als es bei Vollzeitstudierenden üblicherweise der Fall ist. Daher wird davon ausgegangen, dass die strukturelle Machbarkeit einerseits von den Rahmenbedingungen des Studiengangs abhängt und andererseits die private Situation der Studierenden und die Unterstützung des Arbeitgebers in finanzieller, sozialer und zeitlicher Hinsicht eine Rolle spielen. Daher werden für die Erklärung der strukturellen Machbarkeit die folgenden Hypothesen zugrunde gelegt.

H5: Je besser die Qualität der organisationalen Rahmenbedingungen des Studiums, desto höher ist das Maß an struktureller Machbarkeit.

H6: Je stärker die Einbindung des Studierenden durch private und berufliche Verpflichtungen, desto geringer ist das Maß an struktureller Machbarkeit.

H7: Je höher das Maß an Unterstützung seitens des Arbeitgebers, desto höher ist das Maß an struktureller Machbarkeit.

Durch die Kombination von Aspekten der Studienorganisation, der individuellen Studienbedingungen sowie Aspekten der Berufstätigkeit bringt die strukturelle Machbarkeit als Unterfacette zum Ausdruck, inwiefern die Vereinbarkeit von Studium, Beruf und Privatem gelingt.

Es werden hier berufsbegleitende Studiengänge in den Blick genommen, die zu einem akademischen Abschluss führen und im wirtschaftswissen- 
schaftlichen Bereich angesiedelt sind. Die Betrachtungsebene ist der Studiengang als Ganzes. Es werden daher nicht einzelne Lehrveranstaltungen oder Phasen des Studiums betrachtet.

\section{Methodische Vorgehensweise}

Die methodische Vorgehensweise ist in zwei empirische Teilstudien gegliedert. Wie bereits in Abschnitt 3 angesprochen wurde, ist die qualitative Vorstudie, die erste Teilstudie, bereits abgeschlossen. Basierend auf den Ergebnissen der Vorstudie wurde die quantitativ angelegte Hauptstudie konzipiert.

Die in diesem Beitrag fokussierte Pilotierung der Hauptstudie soll dazu dienen, das zuvor ausgearbeitete Modell empirisch zu überprüfen. Dies geschieht im Rahmen einer quantitativen Fragebogenstudie bei berufsbegleitend Studierenden. Die Umfrage erfolgt als Online-Erhebung, mit einer ungefähren Testzeit von 30 Minuten. Im Fragebogen sind Skalen zu der Wahrnehmung der Studierenden der Lehrqualität und der Qualität organisatorischen Rahmenbedingungen enthalten. Darüber hinaus werden die individuellen Lernvoraussetzungen und die Intensität der ArbeitgeberUnterstützung erfasst. Soweit vorhanden, wurden bereits empirisch erprobte Skalen verwendet (Tab. 2). Zusätzlich zu den unten aufgeführten Skalen wurden Informationen zum besuchten Studiengang, $\mathrm{zu}$ den Vorqualifikationen, zur privaten Situation sowie zum sozioökonomischen Status und zur Berufstätigkeit erhoben.

Bei der Konstruktion der Skalen, die das Urteil der Studierenden über den besuchten Studiengang erfassen sollen, wurde darauf geachtet, dass subjektiven Erwartungen der Befragten die Antwort nicht beeinflussen. Die Pilotierung erfolgte im Oktober und November $2015(n=111)$. Die verwendeten Skalen werden gemäß den Ergebnissen der Analysen der Pilotierungsstichprobe für die Haupterhebung (erwartetes $n=400$ ) modifiziert. Mit der quantitativen Studie soll gezeigt werden, inwiefern sich das Forschungsmodell mithilfe des Fragebogeninstruments überprüfen lässt. Bei der Pilotierungsstudie ging es vordergründig darum, die Skalengüte zu überprüfen und zu untersuchen, ob die Modellannahmen grundsätzlich zu bestätigen sind. 
Tab. 1: Übersicht über die eingesetzten Skalen

\begin{tabular}{ll}
\hline Skala (mit Quelle) & Beispielitem \\
\hline $\begin{array}{l}\text { Qualität des Studieneinstiegs } \\
\text { Qualität der zeitlichen }\end{array}$ & $\begin{array}{l}\text { Es gab im Vorfeld Informationsveranstaltungen } \\
\text { über das Studium. }\end{array}$ \\
$\begin{array}{l}\text { Organisation } \\
\text { (Eigenentwicklung) }\end{array}$ & $\begin{array}{l}\text { Die Lehrveranstaltungen sind mit den } \\
\text { Selbststudienphasen zeitlich abgestimmt }\end{array}$ \\
$\begin{array}{l}\text { Beratungsqualität } \\
\text { (Eigenentwicklung) }\end{array}$ & $\begin{array}{l}\text { An meiner Hochschule stehen kompetente } \\
\text { Ansprechpartner für die Beratung zur }\end{array}$ \\
$\begin{array}{l}\text { Studienzufriedenheit } \\
\text { (Eigenentwicklung) }\end{array}$ & $\begin{array}{l}\text { Verfügung. } \\
\text { Wenn ich noch einmal vor der Wahl stehen }\end{array}$ \\
$\begin{array}{l}\text { Lehrqualität } \\
\text { (Adaption Schiefele, Moschner }\end{array}$ & $\begin{array}{l}\text { In den meisten Lehrveranstaltungen gelingt es } \\
\text { den Lehrenden, den Lernstoff gut zu erklären. }\end{array}$ \\
$\begin{array}{l}\text { \& Husstegge, 2002) } \\
\text { Studienmotivation } \\
\text { (Wild, Krapp, Schiefele, }\end{array}$ & $\begin{array}{l}\text { Ich lerne im Studium, weil ich mein Studium } \\
\text { erfolgreich abschließen möchte. }\end{array}$ \\
$\begin{array}{l}\text { Lewalter \& Schreyer, 1995) } \\
\text { Akademisches Selbstkonzept } \\
\text { (Marsh \& O’Neill, 1984, in der } \\
\text { dt. Fassung nach Hußtegge, }\end{array}$ & $\begin{array}{l}\text { In den meisten Lehrveranstaltungen kann ich } \\
\text { mich auf meine Begabung verlassen }\end{array}$ \\
$\begin{array}{l}\text { 2001) } \\
\text { Intensität der Arbeitgeber- }\end{array}$ & $\begin{array}{l}\text { Die Vereinbarkeit von Studium und Beruf wird } \\
\text { pnterstützung }\end{array}$ \\
$\begin{array}{l}\text { pigenentwitiv durch die Unterstützung meines } \\
\text { Arbeitgebers beeinflusst. }\end{array}$ \\
\hline
\end{tabular}

Quelle: Eigene Abbildung

\section{Ergebnisse der Pilotierungsstudie}

Im Folgenden wird auf die Ergebnisse der Pilotierung der Modellüberprüfungsphase eingegangen. Zunächst wird die Zusammensetzung der Stichprobe mit deskriptiven Statistiken beschrieben, die mithilfe von SPSS statistics 23 berechnet wurden. Das Forschungsmodell wurde mithilfe von Strukturgleichungsmodellierung überprüft, wofür das PLS-Verfahren eingesetzt wurde, da es die Möglichkeit bietet, komplexe Zusammenhänge auch bei kleinen Stichprobengrößen zu untersuchen (vgl. Ringle, Sarstedt \& Straub 2012). Die Ergebnisdarstellung nimmt als erstes auf das Messmodell und dann auf das Strukturmodell Bezug. Die zugrundeliegenden Analysen erfolgten mit der Software SmartPLS 2.0. 


\subsection{Beschreibung der Stichprobe}

Die Pilotierung der Messinstrumente erfolgte mit einer Online-Erhebung bei berufsbegleitend Studierenden ( $\mathrm{n}=111$ ). Die Stichprobe setzt sich zu 59\% aus männlichen und zu 38\% aus weiblichen Studierenden zusammen. Studierende, die an Universitäten studieren, machen etwa ein Viertel der Stichprobe aus (26\%), die restlichen $74 \%$ besuchten einen Studiengang an einer (Fach-) Hochschule. Insgesamt sind Beobachtungen von zehn Hochschulen und Universitäten im Datensatz enthalten. Von den $68 \%$ der Studierenden, die einen Bachelorabschluss anstreben, befinden sich die meisten im dritten bis fünften Fachsemester. Bei den 24\% Masterstudierenden ist die Fachsemesterzahl sehr heterogen. Keinen Bachelor- oder Masterabschluss, sondern einen MBA-Abschluss, strebten 8\% der Probanden an. Alle untersuchten Studiengänge sind in den Wirtschaftswissenschaften angesiedelt.

\subsection{Messmodell}

Um zu zeigen, inwiefern die Daten das Modell abbilden können, soll nun die Validität des Messmodells untersucht werden. Dafür werden konvergente und die diskriminante Validität in den Blick genommen (siehe Tabelle 2).

Alle standardisierten Faktorladungen liegen nahe bei oder über dem Grenzwert von .70, was für eine hohe Indikatorreliabilität spricht (vgl. Hulland 1999). Die verwendeten Skalen weisen ein Cronbach's Alpha und eine Composite Reliability von größer .70 auf, so dass die Reliabilität als gut bewertet werden kann (vgl. Bagozzi \& Yi 1988). Anhand der Tatsache, dass bei allen Skalen die durchschnittlich erfasste Varianz über .5 liegt (vgl. Tab. 3), lässt sich eine gute Konvergenzvalidität feststellen (vgl. Bagozzi \& Yi 1988).

Die Ladung der Items zu ihrem zugehörigen Faktor ist jeweils höher als die $\mathrm{zu}$ allen anderen Faktoren, so dass auf ein ausreichendes Maß an diskriminante Validität zu schließen ist (vgl. Chin 1998). Das Messmodell erfüllt somit die Qualitätskriterien und kann für weitere Analysen verwendet werden.

Tab. 2: Kennwerte zu den eigesetzten Skalen

\begin{tabular}{lccccc}
\hline & AVE & CR & CA & MW & SD \\
\hline Lehrqualität & 0.694 & 0.872 & 0.779 & 3.033 & 0.430 \\
Einstiegs-Qualität & 0.656 & 0.884 & 0.825 & 3.049 & 0.618 \\
Zeitmodell-Qualität & 0.656 & 0.850 & 0.750 & 3.131 & 0.583 \\
Beratungs-Qualität & 0.621 & 0.867 & 0.796 & 3.069 & 0.589 \\
\hline
\end{tabular}


Tab. 2: Kennwerte zu den eigesetzten Skalen (Foirtsetzung)

\begin{tabular}{lccccc}
\hline & AVE & CR & CA & MW & SD \\
\hline Extrinsische Motivation & 0.597 & 0.880 & 0.847 & 2.753 & 0.632 \\
Intrinsische Motivation & 0.756 & 0.903 & 0.840 & 2.935 & 0.669 \\
Akadem. Selbstkonzept & 0.614 & 0.888 & 0.843 & 2.784 & 0.530 \\
Intensität AG-Unterstützung & 0.740 & 0.895 & 0.827 & 2.517 & 0.868 \\
Fachliche Machbarkeit & 0.647 & 0.846 & 0.726 & 3.037 & 0.607 \\
Strukturelle Machbarkeit & 0.627 & 0.834 & 0.706 & 3.113 & 0.565 \\
Zufriedenheit & 0.706 & 0.906 & 0.862 & 3.241 & 0.654 \\
\hline
\end{tabular}

Bei allen Konstrukten wurden verbale Ratingskalen mit vier Kategorien verwendet (,,trifft gar nicht $z u$ “ bis ,,trifft voll zu“). AVE=average explained variance, durchschnittlich erklärte Varianz, $C R=$ Composite Reliability, $C A=$ Cronbach's Alpha, $M W=$ Mittelwert, $S D=$ Standardabweichung.

Quelle: eigene Abbildung

\subsection{Strukturmodell}

Um die Signifikanzniveaus des Strukturmodells ermitteln zu können, wurde die Bootstrapping Re-Sampling Methode verwendet, mit der 5000 Stichproben erzeugt wurden (vgl. Hair, Ringle \& Sarstedt 2011). Den Analysen wird ein Signifikanzniveau von 10\% zugrunde gelegt, da im Rahmen der Pilotierung lediglich eine kleine Stichprobe erhoben wurde. Abb. 2 zeigt die mithilfe des PLS-Verfahrens geschätzten Pfadkoeffizienten sowie die mit dem Bootstrapping ermittelten Signifikanzniveaus.

Bezogen auf die formulierten Hypothesen können die folgenden Ergebnisse festgehalten werden: Sowohl $\mathrm{H} 1$ als auch $\mathrm{H} 2$ können bestätigt werden, denn sowohl die fachliche als auch die strukturelle Machbarkeit haben einen signifikant positiven Einfluss auf die Gesamtzufriedenheit mit dem Studium. Zudem zeigt es sich, dass die Zufriedenheit zu einem großen Anteil $\left(\mathrm{R}^{2}=.485\right)$ mithilfe der beiden Machbarkeitsdimensionen erklärt werden kann.

H3 beinhaltete die Annahme, dass die fachliche Machbarkeit auf die Qualität des Lehrangebots zurückzuführen sei. Dieser positiv angenommene Zusammenhang kann bestätigt werden, da die Lehrqualität einen signifikanten positiven Einfluss auf die fachliche Machbarkeit hat.

Der in H4 formulierte Zusammenhang der fachlichen Machbarkeit mit den individuellen Lernvorrausetzungen lässt sich mit dem empirischen Modell nur zum Teil bestätigen. Das akademische Selbstkonzept hat einen positiven, signifikanten Einfluss, die intrinsische sowie die extrinsische 
Motivation haben jedoch keinen signifikanten Einfluss. Auch für die letzte, schulisch erworbene Mathematiknote als Indikator für die kognitiven Lernvoraussetzungen zeigt sich kein signifikanter Einfluss auf die fachliche Machbarkeit. Folglich kann H4 nur für das akademische Selbstkonzept bestätigt werden, da lediglich hier ein signifikanter Zusammenhang vorliegt.

Abb. 2: Empirisches Modell

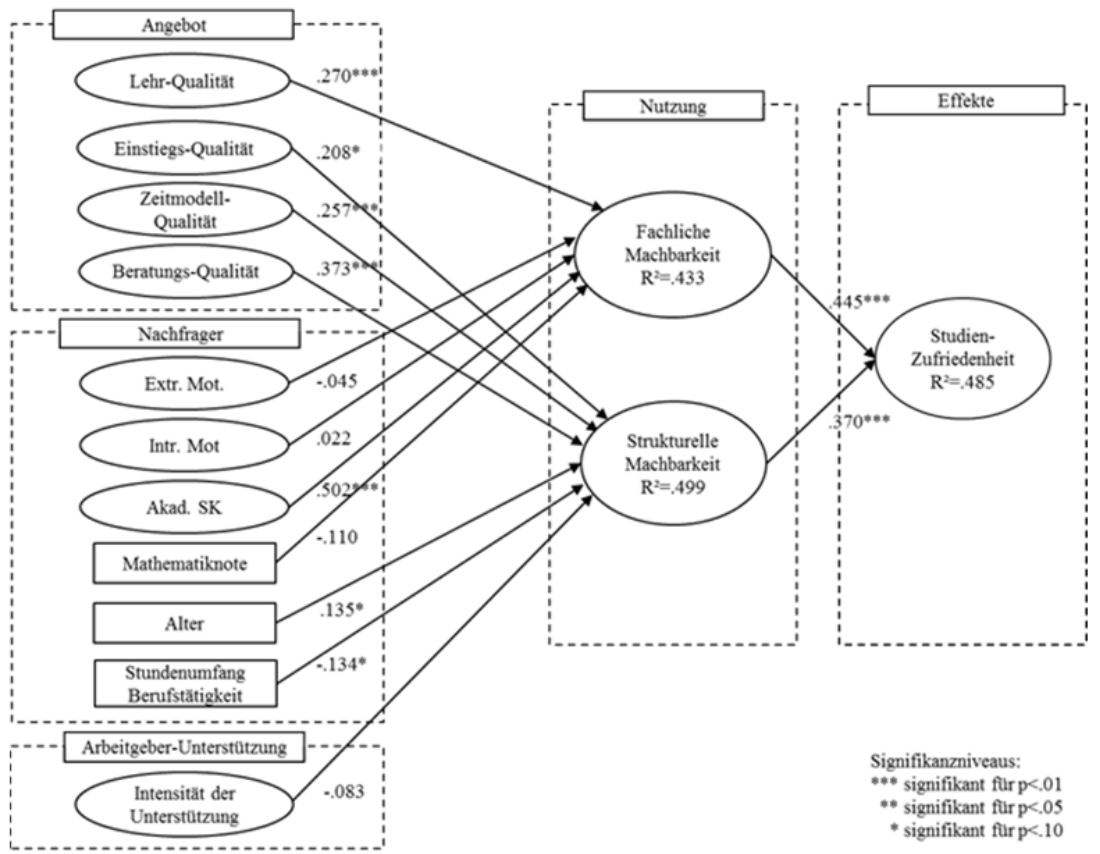

Quelle: eigene Abbildung

Der in H5 angenommene positive Zusammenhang zwischen der wahrgenommenen Qualität der Studienbedingungen und der strukturellen Machbarkeit kann für die Qualität des Studieneinstiegs, des Zeitmodells und der Beratung bestätigt werden. Es kann also davon ausgegangen werden, dass sich die strukturelle Machbarkeit umso besser gestaltet, je besser die Qualität der Studienorganisation wahrgenommen wird.

Zu H6 lässt sich sagen, dass das Alter der Studierenden einen signifikant positiven Einfluss und der wöchentliche Stundenumfang der Berufstätigkeit einen signifikant negativen Einfluss auf die strukturelle Machbarkeit hat. Für den Stundenumfang lässt sich H6 bestätigen, da dieser als Indikator für die Intensität der beruflichen Eingebundenheit gesehen wird. Je stärker die 
Studierenden beruflich eingespannt sind, desto schwieriger gestaltet sich die strukturelle Machbarkeit des Studiums. Für das Lebensalter wurde angenommen, dass ein höheres Lebensalter mit einer stärkeren familiären Eingebundenheit und diese mit einer schlechteren strukturellen Machbarkeit einhergeht. Die Ergebnisse zeigen jedoch den umgekehrten Zusammenhang, so dass H6 nur für die berufliche Eingebundenheit bestätigt werden kann. H7 kann nicht bestätigt werden, da kein signifikanter Einfluss der Intensität der Arbeitgeber-Unterstützung auf die strukturelle Machbarkeit vorliegt.

\section{Diskussion und Ausblick}

Obwohl die Bedeutung berufsbegleitender Studiengänge in den vergangenen Jahren gestiegen ist, bleiben die Studierendenzahlen hinter den Erwartungen zurück. Ein Ansatz, der Erklärungen für die verhältnismäßig geringen Studierendenzahlen liefern kann, ist es, zu untersuchen, wie die Qualität der derzeitigen Studienangebote im berufsbegleitenden Bereich durch die Studierenden beurteilt wird.

Der vorliegende Beitrag liefert daher einen Modellentwurf zur Überprüfung von Qualität in berufsbegleitenden Studiengängen. Dabei finden die durch die Studierenden beurteilte Qualität der Studienorganisation und der Lehrveranstaltungen Berücksichtigung. Deren Einfluss auf die Qualität wird im Zusammenwirken mit individuellen Studierendenmerkmalen und Aspekten der Berufstätigkeit untersucht.

Die Ergebnisse der Pilotierung zeigen, dass die eingesetzten Skalen die Anforderungen hinsichtlich der Gütekriterien erfüllen und für eine weitere Verwendung geeignet erscheinen. Die Ergebnisse bezüglich des Strukturmodells legen nahe, dass die getroffenen Modellannahmen grundsätzlich bestätigt werden können. Die Studienzufriedenheit lässt sich mit den gewählten Teildimensionen fachliche und strukturelle Machbarkeit erklären und die Teildimensionen selbst können mit den Merkmalen des Studiums, der Studierenden und der Berufstätigkeit zu einem hohen Anteil erklärt werden.

Es ist jedoch erkennbar, dass der Einfluss der Studiengangsmerkmale auf die Machbarkeitsdimensionen stärker ist als jener der Studierendenmerkmale, da hier häufiger signifikante Zusammenhänge vorliegen. Eine mögliche Erklärung hierfür ist, dass in den Machbarkeitsdimensionen bereits die Passung zwischen Angebot und Nachfrager enthalten ist und daher die Studiengangskonzeption insoweit auf die Charakteristika der Zielgruppe ausgerichtet ist, dass eine Passung vor allem von ihrer Ausgestaltung bestimmt wird. Weitere Analysen könnten zeigen, welche Studienmodelle zu einer besonders guten Passung führen, so dass Spielräume der Studiengangsentwicklung in Hinblick auf die Machbarkeit des Studiums identifiziert 
werden können. Auf Basis der geringen Beobachtungszahl in der Pilotierungsstichprobe ist dies noch nicht möglich. Es konnten bisher auch nicht alle theoretisch identifizierten Zusammenhänge bestätigt werden. Eine wesentliche Erkenntnis war die Annahme, dass bei berufsbegleitenden Studiengängen neben fachlichen Aspekten auch strukturelle Aspekte sehr bedeutsam für die Qualitätsbeurteilung sind. Dies lässt sich anhand der Ergebnisse bestätigen.

Im dargestellten Modell werden einige mögliche Zusammenhänge zwischen Modellparametern nicht berücksichtigt. Dies gilt z.B. für den Zusammenhang zwischen den kognitiven Lernvoraussetzungen und der strukturellen Machbarkeit des Studiums. Bei den hier beschriebenen Untersuchungen wurde eine Konzentration auf die Haupteffekte versucht, auch wenn Zusammenhänge zwischen fachlichen und strukturellen Aspekte auch denkbar sind.

Bei der Untersuchung der Qualität des Studieneinstiegs wird lediglich die Perspektive der Studierenden berücksichtigt, die tatsächlich ein Studium aufgenommen haben. Es haben jedoch vermutlich weitere Personen Angebote zum Studieneinstieg in Anspruch genommen, sich dann aber doch gegen ein Studium entscheiden. Eine umfassende Untersuchung des Studieneinstiegs müsste das Urteil aller Studieninteressierten berücksichtigen. Dies ist jedoch aus forschungsmethodischer Sicht problematisch, insbesondere aufgrund der schwierigen Kontaktaufnahme, weshalb von einem Einbezug dieser Personen abgesehen wurde. Lohnenswert wäre es auch, die Studierenden über mehrere Semester hinweg mit einer längsschnittlichen Studie zu begleiten. So könnten die Wirkungszusammenhänge zwischen den Studiengangs- und Studierendenmerkmalen detaillierter untersucht werden.

In dem hier beschriebenen Vorhaben werden lediglich wirtschaftswissenschaftliche Studiengänge in den Blick genommen, so dass offen bleibt, ob die Ergebnisse auch für andere Fachrichtungen gelten. Es zeigte sich aber, dass dies bei den angebotenen berufsbegleitenden Studiengängen die am häufigsten vertretene Fachrichtung ist (vgl. Minks et al. 2011). Folglich können die hier gewonnenen Erkenntnisse zwar nicht für alle in Deutschland angebotenen berufsbegleitenden Studiengänge gelten, wohl aber für eine große Gruppe.

Es wird sich zeigen, inwiefern die gefundenen Ergebnisse auch im Zuge der Haupterhebung bei einer größeren Stichprobe (erwartetes $n=400$ ) Gültigkeit aufweisen und inwiefern noch weitere Erkenntnisse zur Erklärung der Studienqualität gewonnen werden können. Anhand dieser Daten sollen die Modellzusammenhänge mit weiterführenden Analysen untersucht werden. Ein Ansatzpunkt hierfür ist der Einbezug weiterer Studiengangsmerkmale, um z.B. Unterschiede zwischen Bachelor- und Masterstudiengängen $\mathrm{zu}$ untersuchen. Es könnten auch Mehrebenenanalysen durchgeführt werden, durch die die gruppierte Struktur der Daten 
berücksichtigt werden kann, sofern die Datenstruktur dies zulassen wird. Darüber hinaus sind weiterführende Analysen zur Wichtigkeit der Faktoren untereinander sowie eine Potenzialanalyse geplant.

\section{Literatur}

Arnold, M., Wetzel, K. \& Dobmann, B. (2014). Erwartungen an die Qualität berufsbegleitender Studiengänge aus Hochschul-und Unternehmensperspektive - eine vergleichende Untersuchung. Beiträge zur Hochschulforschung, 36(4), 64-91.

Autorengruppe Bildungsberichterstattung (Hrsg.) (2014). Bildung in Deutschland 2014. Ein indikatorengestützter Bericht mit einer Analyse zur Bildung von Menschen mit Behinderungen. Bielefeld: Bertelsmann.

Bagozzi, R. P., \& Yi, Y. (1988). On the evaluation of structural equation models. Journal of the Academy of Marketing Science, 16(1), 74-94.

Bargel, T. \& Bargel, H. (2014). Studieren in Teilzeit und Teilzeitstudium. Bielefeld: Universitätsverlag Webler.

Chin, W. W. (1998). The partial least squares approach to structural equation modeling. In G. A. Marcoulides (Ed.), Modern methods for business research (p. 295336). Mahwah, New Jersey: Lawrence Erlbaum Associates.

Hair, J. F., Ringle, C. M., \& Sarstedt, M. (2011). PLS-SEM: indeed a silver bullet. Journal of Marketing Theory and Practice, 19(2), 139-151.

Hulland, J. (1999). Use of partial least squares (PLS) in strategic management research: a review of four recent studies. Strategic Management Journal, 20(2), 195-204.

Hußtegge, R. (2011). Selbstreguliertes Wollen als Bedingung für Studienerfolg an der Universität. Dissertation, Carl von Ossietzky Universität Oldenburg. http://oops. uni-oldenburg.de/1197/2/hussel112.pdf (12.11.2015).

KMK, Kultusministerkonferenz (2009). Hochschulzugang für beruflich qualifizierte Bewerber ohne schulische Hochschulzugangsberechtigung. Beschluss der Kultusministerkonferenz vom 06.03.2009.

Mayring, P. (2010). Qualitative Inhaltsanalyse. Grundlagen und Techniken. Weinheim: Beltz.

Marsh, H.W. \& O'Neill, R. (1984). Self-Description Questionnaire III (SDQ III): The construct validity of multidimensional self-concept ratings by late-adolescents. Journal of Educational Measurement, 21, 153-174.

Minks, K.-H., Netz, N. \& Völk, D. (HIS Hochschul-Informations-System GmbH, Hrsg.). (2011). Berufsbegleitende und duale Studienangebote in Deutschland. Status Quo und Perspektiven. http://www.his.de/pdf/pubfh/fh-201111.pdf. (12. 11.2015).

Nickel, S. \& Duong, S. (2012). Studieren ohne Abitur: Monitoring der Entwicklungen in Bund, Ländern und Hochschulen. CHE Centrum für Hochschulentwicklung: Gütersloh. 
Riggert S. C., Boyle M., Petrosko J. M., Ash D. \& Rude-Parkins C. (2006). Student employment and higher education: Empiricism and contradiction. Review of Educational Research, 76, 63-92

Ringle C. M., Sarstedt M. \& Straub D. W. (2012). A critical look at the use of PLSSEM in MIS Quarterly. MIS Quarterly, 36(1), iii-xiv.

Schiefele, U., Moschner, B. \& Husstegge, R. (2002). Skalenhandbuch SMILE-Projekt. Bielefeld: Universität Bielefeld, Abteilung für Psychologie.

Schiefele, U. , Streblow, L.; Ermgassen, U. \& Moschner, B. (2003). Lernmotivation und Lernstrategien als Bedingungen der Studienleistung. Ergebnisse einer Längsschnittstudie. Zeitschrift für pädagogische Psychologie, 17(3/4), 185-198.

Schuetze, H. G. \& Slowey, M. (2000). Higher Education and Lifelong Learners. International Perspectives on Change. London: Routledge.

Teichler, U. \& Wolter, A. (2004). Zugangswege und Studienangebote für nichttraditionell Studierende. Die Hochschule, 2004 (2), 64-80. http://www.hof.unihalle.de/journal/texte/04_2/Teichler_Zugangswege_und_Studienangebote.pdf (10.4.2016).

Thiel, F., Veit, S., Blüthmann, I., Lepa, S. \& Ficzko, M. (2008). Ergebnisse der Befragung der Studierenden in den Bachelorstudiengängen an der Freien Universität Berlin - Sommersemester 2008. http://www.fu-berlin.de/universitaet/entwicklung/qualitaetsmanagement/bachelorbefragung/bachelorbefragung2008.pdf?1304061426 (10.4.2016).

Wild, K.-P., Krapp, A., Schiefele, U., Lewalter, D. \& Schreyer, I. (1995). Dokumentation und Analyse der Fragebogenverfahren und Tests (Berichte aus dem DFGProjekt „Bedingungen und Auswirkungen berufsspezifischer Lernmotivation“, Nr. 2). München: Universität der Bundeswehr, Institut für Erziehungswissenschaft und Pädagogische Psychologie.

Wolter, A. (2000). Non-traditional students in German higher education - Situation, profiles, policies and perspectives. In H. G. Schuetze \& M. Slowey (Eds), Higher Education and Lifelong Learners. International Perspectives on Change (p. 4866). London: Routledge.

Wolter, A., Banscherus, U., Kamm, C. \& Otto, A. (2014). Durchlässigkeit zwischen beruflicher und akademischer Bildung als mehrstufiges Konzept: Bilanz und Perspektiven. Beiträge zur Hochschulforschung, 36(4), 8-39.

Wolter, A. \& Geffers, J. (2013). Zielgruppen lebenslangen Lernens an Hochschulen Ausgewählte empirische Befunde. https://de.offene-hochschulen.de/public_libraries/1 (12.11.2015).

Zawacki-Richter, O., von Prümmer, C. \& Stöter, J. (2015). Open Universities: Offener Zugang zur Hochschule in nationaler und internationaler Perspektive. Beiträge zur Hochschulforschung, 37(1), 8-25.

Zinn, B. (2012). Ein ingenieurwissenschaftliches Studium von beruflich qualifizierten Studierenden - Chancen und Risiken. Zeitschrift für Berufs- und Wirtschaftspädagogik, 108(2), 273-290. 


\title{
Fachdidaktisches Wissen im Rechnungswesen - Befunde einer Onlinebefragung aus Österreich
}

\author{
Christoph Helm, Stefanie Antonia Mayer
}

\section{Einleitung}

Das Wissen darüber, welche Fähigkeiten von Lehrpersonen besonders relevant für den Lernerfolg von Schüler/inne/n sind, ist notwendig, um die Lehreraus- und -fortbildung weiterentwickeln zu können. Dass das Professionswissen von Lehrkräften eine wichtige Voraussetzung für effektiven Unterricht ist, ist unumstritten. So konnte die COACTIV-Studie (Professionswissen von Lehrkräften, kognitiv aktivierender Mathematikunterricht und die Entwicklung mathematischer Kompetenz) indirekte Effekte des fachdidaktischen Wissens von Mathematiklehrkräften (mediiert über die kognitive Aktivierung im Unterricht) auf die Schülerleistungen nachweisen (vgl. Baumert et al. 2010). Während auch für die Naturwissenschaften (bspw. Cauet, Liepertz, Kirschner, Borowski \& Fischer 2015) und dem Bereich Lesen (bspw. Kelcey 2011) bereits Befunde vorliegen, steht die Forschung im berufsbildenden Bereich erst am Anfang. So wurden für die Domäne Rechnungswesen (RW) erste Arbeiten zur Erfassung des fachdidaktischen Wissens von Wirtschaftspädagogikstudent/inn/en (z.B. Schnick-Vollmer et al. 2015; Mindnich, Berger \& Fritsch 2013) vorgelegt. Untersuchungen zu Effekten dieses Professionswissens auf die Schülerleistungen in RW sind dagegen erst in Planung.

Der vorliegende Beitrag knüpft an diese Arbeiten an, fokussiert dabei aber auf österreichische RW-Lehrpersonen. Wir wollen mit der Erfassung des fachdidaktischen Wissens von RW-Lehrkräften in Österreich einen ersten Schritt setzen, der für das deutsche Berufsbildungssystem bereits sehr aufwendig (u.a. auch mit Videostudien, Berger et al. 2013) gegangen wurde. Der Einsatz des bereits existierenden Testinstrumentes der Mannheimer, Frankfurter und Darmstädter Forschergruppe (z.B. Berger et al. 2013) war aus mehreren Gründen nicht möglich. Einerseits war das Testinstrument nicht zugänglich, da die Projektphase noch nicht abgeschlossen war. Andererseits wäre der Einsatz aufgrund organisatorischer und ressourcenbedingter Hürden nur schwer möglich gewesen. Lehrpersonen hätten sich freiwillig einem Papier-Bleistift-Assessment unterziehen müssen, das vor Ort durchgeführt werden hätte müssen. Aus diesen Gründen versucht die vorliegende Studie das fachdidaktische Wissen (im Folgenden kurz: PCK; Pedagogical Content Knowledge) mit einem Onlineinstrument zu erfassen, das in Teilen auf dem 
Prinzip der „Übereinstimmung mit Expertenurteilen“ (vgl. Maag Merki, Ramseier \& Karlen 2013) basiert. Die vorliegende Studie geht daher folgender Frage nach:

Inwiefern ist es möglich, das fachdidaktische Wissen von österreichischen $R W$-Lehrkräften mit einem Onlineinstrument zu erfassen?

Die Relevanz der Erforschung dieser Frage kann in folgenden Punkten gesehen werden:

(1) Die Auseinandersetzung mit dem Thema ist sowohl für Hochschullehrende, Wirtschaftspädagog/inn/en und für Schüler/innen relevant, da diese Forschung weitere Mosaiksteinchen zum komplexen Bild über das Lehren und Lernen im RW-Unterricht liefert.

(2) Die Erkenntnisse allgemeinbildender Domänen müssen nicht auch für die Berufsbildung gelten. Für das Fach Rechnungswesen gilt die Mathematik zwar als zentrale Hilfsdomäne, jedoch ist der RW-Unterricht - wie jeder Fachunterricht - durch domänenspezifische Charakteristika geprägt (z.B. Übungsintensität: Götzl, Jahn \& Held 2013; Dominanz von Lernaufgaben: Seifried 2004).

(3) Die Fokussierung auf österreichische Lehrkräfte erscheint notwendig, da sich die österreichische Ausbildung von Wirtschaftspädagog/inn/en sowie ihr Arbeitsfeld bedeutend von jenen in Deutschland unterscheidet: (a) einphasige vs. zweiphasige Lehrerausbildung (vgl. Fritsch et al. 2015), (b) sekundäre vs. post-sekundäre Berufs(aus)bildung (vgl. Lassnig 2016) und (c) kaufmännische Vollzeitschule vs. duale Ausbildung.

Obwohl das Pedagogical Knowledge und das Content Knowledge mit dem PCK korreliert sind, sind sie nicht Teil der vorliegenden Studie. Wir blenden die beiden anderen Facetten aus, weil sie zwar wichtige, aber nicht hinreichende Voraussetzungen für erfolgreichen Unterricht sind (vgl. Neuweg 2011). Ebenso können Effekte des RW-PCKs auf die Unterrichtsgestaltung und die kognitiven und non-kognitiven Schüleroutputs mangels Analysen noch nicht berichtet werden.

\section{Facetten und Befunde zum fachdidaktischen Professionswissen im Rechnungswesen}

Shulman $(1987,8)$ definiert das fachdidaktische Wissen (PCK) allgemein als „that special amalgam of content and pedagogy that is uniquely the province of teachers, their own special form of professional understanding". Wirft man einen Blick auf die Operationalisierung des PCKs in unterschiedlichen Fach- 
didaktiken, so ist festzustellen, dass sich die Definitionen sehr ähneln. Im Rahmen der COACTIV-Studie zählen Baumert und Kunter (2006, 495ff.) das Wissen über (a) das Potenzial von mathematischen Aufgaben, (b) mathematische Schülervorstellungen (z.B. typische Fehler) und (c) verschiedene Repräsentations- und Erklärungsmöglichkeiten zum fachdidaktischen Wissen in Mathematik. In den Naturwissenschaften wurde das fachdidaktische Wissen bspw. im Rahmen der ProwiN-Studie (Professionswissen in Naturwissenschaften) als das fachdidaktische Wissen über die Vermittlung fachspezifischer Konzepte im Bereich Mechanik, über Schülerkognitionen sowie über die Durchführung physikalischer Experimente definiert und empirisch erfasst (vgl. Caut et al. 2015). In der QuiP-Studie (Quality of Instruction in Physics) dagegen wurden die Dimensionen Schülerfehlvorstellungen (z.B. Anführen von Gründen für eine bestimmte Schülerantwort), Curriculum (z.B. Zuordnung bestimmter Curriculuminhalte zu bestimmten Schulstufen) und schwierige Inhaltsbereiche (z.B. Identifikation von Lernschwierigkeiten bei vorgelegten Schulbuchausschnitten) untersucht (vgl. Ergönenç, Neumann \& Fischer 2014). Auch in weniger strukturierten Fächern wie den Sprachen wurden bereits Versuche zur Erfassung des PCKs unternommen. In der TEDSLT-Studie (Teacher Education and Development Study: Learning to Teach) wurde das fachdidaktische Wissen in Englisch über folgende Inhaltsbereiche erfasst: Wissen über (1) fremdsprachliche Lehr- und Lernprozesse (z.B. Vermittlungsstrategien), (2) Curriculares Wissen (z.B. über Lehrmaterialien) und (3) Verstehensprozesse (z.B. Schülerfehlvorstellungen) (vgl. Roters, König, Tachtsoglou \& Nold 2013). Vergleicht man die Definitionen sowie die empirische Erfassung des fachdidaktischen Wissens in den unterschiedlichen Domänen, sieht man deutlich, dass sich die Modelle sehr ähneln. Dieser Konzeption folgend wurde auch in der Domäne Rechnungswesen (z.B. Berger et al. 2013) eine Unterteilung in drei Facetten vorgenommen (siehe die diagonale Achse in Abbildung 1):

1. Wissen über Schülerkognitionen: Das Bennen und Erklären von (typischen) Schülerfehlvorstellungen, -fehlern und Fehlerursachen sowie Verständnisproblemen oder Bearbeitungsschwierigkeiten sind zentrale Merkmale dieser Facette. Beispiel: Die Lehrkraft kann die Ursache für einen Fehler in einer Schülerlösung analysieren.

2. Wissen über das Zugänglichmachen von Inhalten: Das Erklären und Veranschaulichen eines Inhaltsbereiches / eines RW-spezifischen Konzepts anhand verschiedener Zugangsmöglichkeiten (hier z.B. grafisch, in Prosa, verbal, plastisch, aktionistisch, ...) gehören zum Lehrerwissen dieser Facette. Beispiel: Die Lehrkraft kann verschiedene Erklärungen zu einem Sachverhalt generieren.

3. Wissen über das Potenzial von Aufgaben: Das Erkennen und Beurteilen des kognitiven Aktivierungspotenzials einer Aufgabe bilden Fähigkeiten 
dieser Facette ab. Beispiel: Die Lehrkraft kann beurteilen, ob das Vorwissen der Schüler/innen für die Bearbeitung einer Rechnungswesenaufgabe ausreichend ist.

Die letzte Facette wird als besonders zentral für den RW-Unterricht erachtet, da in Hinblick auf seine besondere Struktur der Einsatz von Aufgaben eine zentrale Dimension der Unterrichtsqualität sowie ein Mittel zur Steuerung kognitiv anspruchsvoller Lernprozesse darstellt (vgl. Berger et al. 2013, 96).

Im vorgestellten Modell spielen über diese drei PCK-Facetten hinaus die Inhaltsbereiche und das Anforderungsniveau (Aufgabenschwierigkeit) eine wesentliche Rolle für die Testkonstruktion. Abbildung 1 zeigt auf der horizontalen Achse die Lerninhaltsbereiche des Rechnungswesenunterrichts, welche von Mindnich et al. (2013) durch eine Analyse der Lehrpläne eruiert wurden. Auf der vertikalen Achse wird die Schwierigkeit der jeweiligen Items repräsentiert. Diese unterteilt sich in das kognitive Anspruchsniveau (z.B. Reproduktion) sowie die quantitative (z.B. Verknüpfung von Wissensbereichen) und qualitative Komplexität (z.B. buchhalterische Operationen).

\section{Forschungsbefunde}

Die COACTIV-Studie (vgl. Baumert et al. 2010) hat eindrucksvoll die Bedeutung des Professionswissens von Lehrpersonen im Fach Mathematik belegt. Die Ergebnisse zeigen, dass das fachdidaktische Wissen von Lehrpersonen zu einem erheblichen Teil die kognitive Struktur der Lerngelegenheiten bestimmt (vgl. Baumert \& Kunter 2011, 185). Darüber hinaus ist das fachdidaktische Wissen eindeutig leistungsrelevant. Dies zeigt der Regressionskoeffizient von $\beta=.62$ zur Vorhersage der Mathematikleistung am Ende der 10. Klasse durch das PCK von Lehrkräften (vgl. Baumert \& Kunter 2011, 181). So können 39\% der Leistungsvarianz zwischen den Klassen alleine durch das fachdidaktische Lehrerwissen erklärt werden.

Im allgemeinbildenden Bereich setzen sich auch die TEDS-M- sowie TEDS-LT-Studien in den Domänen Mathematik, Deutsch und Englisch (vgl. Blömeke 2013) sowie die ProwiN-Studie in der naturwissenschaftlichen Domäne Physik (vgl. Cauet et al. 2015) mit der Modellierung und Messung des Professionswissens auseinander. In Letzterer gelang es bisher allerdings noch nicht die Wirkungskette Professionswissen $>$ kognitive Aktivierungsqualität des Unterrichts $>$ Schülerleistungen zu belegen (vgl. Cauet et al. 2015). Dies relativiert die durch die Ergebnisse der COACTIV-Studie aufgebauten Erwartungen an die Effekte des Lehrerwissens in anderen Domänen.

Im berufsbildenden Bereich ist die ILLEV-Studie zu nennen, die das wirtschaftswissenschaftliche Wissen von BWL- und WirtschaftspädagogikStudierenden erfasst, um die Effekte der unterschiedlichen Studienmodelle (Bachelor, Master und Diplom) auf die Professionalität zu untersuchen (vgl. Förster \& Zlatkin-Troitschanskaia 2010, 106f.). Die Ergebnisse dieser Studie 
zeigen einerseits, dass es gelang das ökonomische Wissen von Wirtschaftspädagogikstudierenden valide zu erfassen, und andererseits, dass sich Bachelorstudierende hinsichtlich ihrer Fachkompetenz kaum von Diplomstudierenden unterscheiden (vgl. Förster \& Zlatkin-Troitschanskaia, 2010, 119).

\section{Abb. 1: Modell zum fachdidaktischen Wissen in Rechnungswesen}

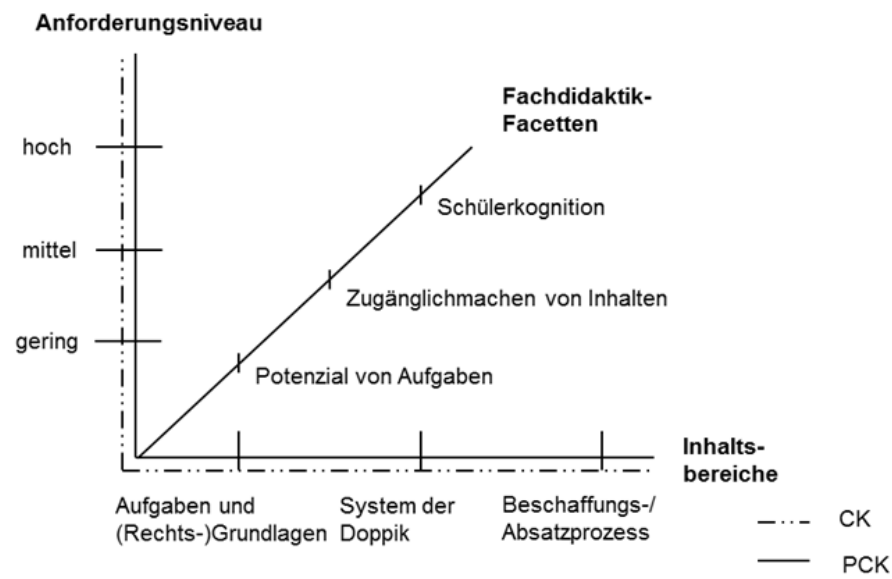

Quelle: Berger et al. 2013, 95

Für die vorliegende Studie ist das KoMeWP-Projekt (Kompetenzmessung im wirtschaftspädagogischen Studium) besonders interessant, da es das Ziel verfolgt die fachlichen und fachdidaktischen Fähigkeiten von Studierenden zu modellieren (vgl. Mindnich et al. 2013). Dazu wurde ein Paper-PencilTest entwickelt, der das CK und das PCK im externen Rechnungswesen erfasst. Schnick-Vollmer et al. $(2015,28)$ untersuchten mit diesem Testinstrument u.a. den Zusammenhang zwischen Fachwissen und fachdidaktischem Wissen. Die latente Korrelation zwischen diesen beiden Variablen fällt mit $.92 \mathrm{sehr}$ hoch aus. Dies bedeutet, dass 85\% der Varianz in den CKund PCK-Leistungen der Studierenden deckungsgleich sind und sich somit diese Kompetenzen empirisch kaum voneinander trennen lassen.

Trotz des Vorliegens dieses Instruments musste in der hier präsentierten Studie ein anderes Verfahren eingesetzt werden, da der KoMeWP-Test sich einerseits noch in der Entwicklungsphase befand und andererseits die aufwendige Durchführung (eine österreichweite Face-to-Face-Prüfung der RWLehrpersonen mittels eines 45minütigen Papier-Bleistift-Testung) vom Autor bzw. der Autorin nicht geleistet hätte werden können. Aus diesen Gründen wird in den folgenden Abschnitten ein Onlineinstrument zur Erfassung des RW-PCKs sowie die Ergebnisse einer Dimensions- und Validierungsstudie 
vorgestellt. Der Einsatz des oben beschriebenen Instrumentes bei österreichischen Lehrpersonen wäre dennoch hoch spannend, da ein Onlineinstrument mit vielen Nachteilen verbunden ist (siehe die Diskussion).

\section{Methode}

\subsection{Design der Studie und Beschreibung der Stichprobe}

Im Mai 2015 wurden österreichweit Lehrkräfte aus berufsbildenden mittleren und höheren Schulen eingeladen einen Onlinetest bzw. -fragebogen auszufüllen. Von den 524 eingeladenen Kommerzialist/inn/en antworteten 91 (32,9\% männlich, Alter: $M=47,6, \mathrm{SD}=7,9)$. Dies entspricht einer Rücklaufquote von $17,4 \%$, was aufgrund der stetigen beruflichen Belastung von Lehrkräften deutlich über unseren Erwartungen liegt und für die Teilnahmemotivation der Lehrpersonen spricht. 20 der 91 Lehrpersonen unterrichten zudem Schulklassen, die im Rahmen der LOTUS-Studie (vgl. Helm 2015) zur Wahrnehmung der Instruktionsqualität des Unterrichts befragt wurden, sodass für diese Lehrpersonen eine Gegenüberstellung der Schülersicht $(\mathrm{N}=519,29,3 \%$ männlich, Alter: $\mathrm{M}=14,45, \mathrm{SD}=0,74)$ möglich ist.

\subsection{Operationalisierung des $R W$-PCKs sowie der von Schüler/inne/n wahrgenommenen Instruktionsqualität}

In Anlehnung an das Theoriemodell von Berger et al. (2013) wurden Fragebogenitems entwickelt, die den drei Facetten des RW-PCKs zuordenbar sind. In Summe wurden sieben Fragenblöcke mit Performance- und Selbsteinschätzungsitems erstellt. Drei Fragenblöcke wurden mit offenem Antwortformat vorgelegt. Die vollständigen Itemtexte und die Itemzuordnung zu den PCK-Facetten können im Netz nachgesehen werden: www.edumetrics.delforschungsprojekte. Die mittlere Bearbeitungszeit betrug rund 28 Minuten. Der Tabelle 2 sind Angaben zur Güte der Skalen (Schwierigkeiten, Trennschärfen und Reliabilitäten) zu entnehmen.

\section{Wissen über Schülerkognitionen}

(Block 1) Wissen über Schülerfehlvorstellungen: Den Lehrpersonen wurden drei Verständnisfragen von Schüler/inne/n beim Lernen im Fach Rechnungswesen vorgelegt, die typische Fehlvorstellungen (Skonto, Rückstellung, Zahlung auf Ziel) repräsentieren, bspw.: ,Sie stellen den Schüler/inne/n im Unterricht folgende Frage: ,Warum bildet ein Unternehmen Rückstellungen?' Daraufhin gibt Ihnen Susanne folgende Antwort: „Rückstellungen 
werden gebildet, um für drohende Verluste genug Geld zu haben, d.h. wir legen uns den entsprechenden Geldbetrag zur Seite. "Die Lehrpersonen wurden aufgefordert, so ausführlich wie möglich anzugeben, worin der Denkfehler liegen könnte. Die Antworten wurden entsprechend ihrer Zeichenlänge (I) und ihres Informationsgehaltes sowie ihrer Korrektheit (II) von zwei Ratern kodiert $\left(\mathrm{ICC}_{\text {just, fixed }}=.86\right)$. Allerdings waren die beiden weiteren Fehlvorstellung-Items zum Skonto und zur Zahlung auf Ziel zu leicht gestaltet, sodass nahezu alle Lehrpersonen den Denkfehler identifizieren und Erklärungen geben konnten. Die beiden Items wurden ausgeschieden.

(Block 2) Kenntnisse über mögliche Fehlerarten: Das Wissen über häufige Fehlerquellen kann als eine zentrale Facette der professionellen Fehlerkompetenz angesehen werden (vgl. Wuttke \& Seifried 2012). Um diese zu erfassen, mussten die Lehrpersonen für zwölf Themenbereiche der Buchführung - deren Schwierigkeitsgrad von Seifried $(2004,49)$ bei kaufmännischen Berufsschüler/inne/n empirisch festgestellt wurde - einschätzen, wie häufig sie Schüler/inne/n Lernschwierigkeiten bereiten. Die sich daraus ergebende Rangordnung wurde mit jener von Seifried in Beziehung gesetzt. Die so ermittelten Korrelationen bilden im Sinne der Fehlerkompetenz von Lehrpersonen einen weiteren Indikator für das Wissen über Schülerkognitionen.

(Block 3) Kenntnisse über Lösungswege/-strategien (Skills): Das Wissen über Skills (Lösungsschritte), die beim Bearbeiten von Aufgaben durch Schüler/innen angewandt werden müssen, ist ebenfalls Ausdruck des PCKs und spiegelt insbesondere das Wissen über Schülerkognitionen wider. Diese Skills wurden von Helm, Bley, George und Pocrnja (2015) für das Verbuchen eines Geschäftsfalles analysiert. Es konnten zehn Skills für die Verbuchung des Skontos identifiziert werden (z.B. Erfassen der Situation und Übersetzung ins RW; Korrektur der Steuer; ...). Die Lehrpersonen der vorliegenden Stichprobe wurden gebeten, die zentralen Skills von Schüler/inne/n beim Verbuchen des Skontos anzuführen. Zwei Rater kodierten und verglichen die

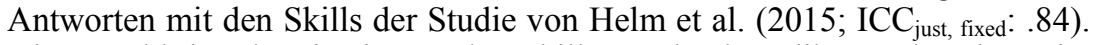
Die Anzahl der übereinstimmenden Skills wurde als Indikator über das Wissen von Schülerkognitionen herangezogen.

\section{Wissen über das Potential von Aufgaben}

(Block 4) Wissen über das kognitive Aktivierungspotential von Aufgaben: Die Lehrpersonen wurden gebeten anzugeben, nach welchen Kriterien sie Aufgaben für den Rechnungswesenunterricht auswählen bzw. erstellen. Die offenen Antworten wurden mit wissenschaftlichen Kriterienkatalogen zur Beurteilung der fachdidaktischen Qualität von Aufgaben (vgl. Fortmüller, Kreilinger, Rendl, Mohr \& Steinbach 2013; Maier, Kleinknecht, Metz \& Bohl 2010; Mayer 2015) abgeglichen. Diese Kataloge basieren auf allgemeindidaktischen Lernzieltaxonomien und Befunden der Kognitionspsychologie sowie fachdidaktischen Überlegungen zum RW; in Summe geben sie 
13 Kriterien vor (bspw. Anzahl der Wissenseinheiten, Lebensweltbezug, fachliche Korrektheit, ...). Die Übereinstimmungshäufigkeiten wurden als Wissensindikatoren herangezogen (I). Darüber hinaus wurde die Anzahl der genannten Kriterien - unabhängig davon, ob sie in den Kriterienkatalogen genannt werden - als zweiter Indikator für diese Wissensfacette über Lernaufgaben herangezogen (II).

(Block 5) Diagnose von Aufgabenschwierigkeiten: Diese Fähigkeit, die gleichzeitig auch Ausdruck des Wissens über Schülerkognitionen ist und daher auch weiter oben angeführt werden könnte, lässt sich als Bestandteil des Lehrerwissens über das Potential von Aufgaben ansehen, da es darum geht festzustellen, wie gut es Lehrpersonen gelingt, die Schwierigkeit (und daher das Anforderungsniveau für Schüler/innen) von Lernaufgaben akkurat einzuschätzen (vgl. van Ophuysen 2010). Zur Erfassung der Diagnosefähigkeit wurden die Lehrpersonen gebeten, auf einer Skala von -5 (sehr leicht) bis +5 (sehr schwer) zehn typische Schulbuchaufgaben (Geschäftsvorfälle, die verbucht werden müssen) einzuschätzen. Für dieselben zehn Items liegen aus der LOTUS-Studie (vgl. Helm in Druck) Itemschwierigkeiten (basierend auf dem Rasch-Modell) vor. Durch Gegenüberstellung der Lehrerurteile und den empirischen Itemschwierigkeiten war es möglich die Niveau-, Differenzierungs- und Rangkomponente der pädagogisch-diagnostischen Fähigkeit (vgl. PDK, van Ophuysen 2010) zu berechnen. Diese drei Indikatoren bilden weitere Analysevariablen, die über das PCK-Modell hinausgehen.

\section{Wissen über das Zugänglichmachen von Inhalten}

Das Wissen über das Zugänglichmachen von Inhalten wurde über drei Frageblöcke mit 5-stufigem Antwortformat erfasst. Block 6 fragte die Lehrpersonen nach alternativen Erklärungs- bzw. Darstellungsformen, die von ihnen im Unterricht eingesetzt werden. Die Antworten ließen sich grob in drei Gruppen einteilen: traditionelle (z.B. Einsatz von T-Konten, Belegen, Tabellen und Zeitlinien), praxisorientierte (z.B. Einsatz von Geschäftsfällen aus der Praxis und plastischem Material) sowie visuelle und aktionistische (z.B. Visualisierung von Zusammenhängen mittels Farben, Einsatz von Bildern und Rollenspielen) Darstellungsformen. Indikator (I) enthält die Anzahl dieser Kategorien zu denen sich die Nennungen zuordnen lassen. Indikator (II) enthält die Anzahl der Nennungen insgesamt.

Der Block 7 beinhaltet Indikatoren über das Wissen über (fach-)didaktische Modelle. Auch wenn dieses Wissen nicht Bestandteil des oben angeführten PCK-Modells nach Berger et al. (2013) ist, so stellt das Wissen über didaktische Modelle eine Grundvoraussetzung für das Zugänglichmachen von Inhalten dar. Gerade Planungs- und Analysekonzepte wie die didaktische Analyse nach Klafki (1958) oder das Phasenmodell problemorientierten Unterrichts nach Seel (1974) zwingen Lehrpersonen sich Gedanken über die Zugänglichkeit der Unterrichtsinhalte für die zu unterrichtende Schülergrup- 
pe zu machen. Block 7 setzt sich aus acht bzw. sechs vorgelegten allgemeinbzw. fachdidaktischen Modellen (z.B. didaktische Analyse nach Klafki bzw. Wirtschaftsinstrumentelles RW) zusammen. Die Lehrpersonen wurden aufgefordert anzugeben, ob sie die Modelle kennen bzw. anwenden $(1=$,Nicht bekannt" ... $3=$,Gut darüber informiert“ .. 5 = „Wende ich an“). Auch hier wurden die aufsummierten Antworten als Indikatoren für diese Wissensform herangezogen. Die internen Konsistenzen dieser Skalen sind zufriedenstellend: $\alpha .75$ allgemeindidaktisch / $\alpha .80$ fachdidaktisch.

\section{Außenkriterien}

Um die Validität der oben angeführten Items näherungsweise zu prüfen, wurden mehrere Außenkriterien erfasst. Es ist darauf hinzuweisen, dass wir mit „Außenkriterium“ nicht die Erfassung des PCKs mittels weiterer Testinstrumente meinen, sondern mit Bühner (2011, 63) Kriterien, „mit denen der Test aufgrund seines Messanspruchs korrelieren sollte“. So gehen wir davon aus, dass die mit dem Onlinefragebogen erfassten Lehrerwissensindikatoren mit der Anzahl der jährlich besuchten fachdidaktischen Fortbildungen, der Berufserfahrung in der Wirtschaft sowie dem didaktischen Handeln (Selbstund Fremdeinschätzung) im Zusammenhang stehen. Dies vermutlich nur schwach, da jeweils das Lehrerwissen und -handeln noch von vielen weiteren Faktoren (z.B. Lehrersichtweisen, Seifried 2009) beeinflusst ist. Auch wird im Folgenden mit der Schülerperspektive nicht das PCK der Lehrpersonen beurteilt, vielmehr dient die Schülerperspektive als ergänzendes Validitätskriterium: Nach Cauet, Borowski \& Fischer (2016) werden Testinstrumente oft ohne Bezug zu Kriterien guten und erfolgreichen Unterrichts versucht zu validieren. Aus ihrer Sicht ist damit aber eine valide Interpretation der Testwerte nicht sichergestellt. Daher stellt die Schülereinschätzung zur Qualität des Unterrichts (hier die Merkmale kognitive Aktivierung und Verstehenspotential) ein wichtiges Außenkriterium dar.

In Block 9 wurde einerseits nach der Anzahl der jährlich besuchten fachdidaktischen Fortbildungen gefragt, da sich nach Lipowsky (o.J., 52) ,erfolgreiche und wirksame Fortbildungen durch einen eher engen fachdidaktischen Fokus" auszeichnen. Fachdidaktische Fortbildungen stellen für Lehrpersonen eine der wenigen Opportunities to Learn (OTL) dar, die nachweislich im signifikanten Zusammenhang mit dem fachdidaktischen Wissen von Lehrkräften stehen (z.B. für Mathematik: Blömeke 2010). Andererseits wurde die Berufserfahrung in der Wirtschaft in Jahren erfasst, da die kaufmännische Praxis zentrale Grundlage dafür ist, Praxiserfahrungen positiv in die Unterrichtsgestaltung einfließen zu lassen (vgl. Weinfurtner 2009).

Als weiteres Außenkriterium wurde auch die Selbsteinschätzung der Lehrpersonen in Hinblick auf ihr didaktisches Handeln erfasst (Block 10). Die Lehrpersonen wurden aufgefordert auf einer fünfstufigen Skala (,trifft nicht zu“ bis „trifft voll zu“) Items zu ihrer (1) Kompetenzorientierung im 
Unterricht (5 Items, Beispielitem: „Ich arbeite im RW-Unterricht mit Kompetenzrastern.“, $\alpha$.65), (2) Individualisierungskompetenz (4, ,Bei der Unterrichtsplanung versuche ich die Interessen meiner Schüler/innen zu berücksichtigen.“, $\alpha$.71) und (3) Diagnosefähigkeit (4, „Bei den meisten Schulbuchaufgaben weiß ich sofort, ob diese Aufgabe den Schüler/inne/n schwer bzw. leicht fällt.“, $\alpha$.59) einzuschätzen.

Als letztes Validierungskriterium wird die Fremdeinschätzung des Lehrerhandelns durch die Schüler/innen mit Fokus auf die kognitive Aktivierung im Unterricht (21 „Im RW-Unterricht vergleichen wir unterschiedliche Lösungswege."; $\alpha$.88, ICC(1): .31, ICC(2): .90; zur Berechnung siehe Lüdtke, Trautwein, Kunter \& Baumert 2006) und auf das Verstehen im Unterricht (9, „Unsere RW-Lehrperson kann gut erklären.“; $\alpha$.90, $\operatorname{ICC}(1)$ : .34, ICC(2): .92) (Block 11) erhoben. Diese Fremdeinschätzung liegt nur für die oben beschriebene Teilstichprobe der LOTUS-Studie vor. Es ist wichtig anzumerken, dass es hierbei nicht um die Einschätzung des fachdidaktischen Wissens der Lehrperson durch die Schüler/innen geht, sondern diese Schülereinschätzung einen Indikator für die Erklärungskompetenz der Lehrperson darstellt. Wer, wenn nicht die Adressat/inn/en der Lehrerklärungen könnten besser einschätzen, wie verständlich Lehrer/innen erklären können?

\subsection{Analyseverfahren und Prüfung der Normalverteilung}

Zur Durchführung der Dimensions- und Validitätsanalysen werden die gesammelten Daten Korrelationsanalysen unterzogen. Die Voraussetzungsprüfung ergab wie erwartet, dass die Indikatoren Wissen über Fehlvorstellung (I), kognitives Aktivierungspotential von Lernaufgaben, (fach)didaktische Modelle sowie fachdidaktische Fortbildung signifikant rechtsschief verteilt sind. D.h., hier liegen vor allem Ausprägungen im unteren Skalen- bzw. Wissensbereich vor. Dagegen erweist sich die Verteilung der Diagnosefähigkeit (Rangkomponente) als linksschief, was bedeutet, dass die Mehrzahl der Lehrpersonen die Lernaufgaben relativ gut in Hinblick auf ihre Schwierigkeit reihen konnten. Bei den Indikatoren Skills und Darstellungsformen liegt eine bipolare Verteilung vor, d.h. die mittleren Ausprägungen sind relativ gering besetzt. Diese Hinweise sind bei der Interpretation der nachfolgend dargestellten Korrelationen zu berücksichtigen. Der Anteil fehlender Werte lag im höchsten Fall bei 5 fehlenden Angaben (5,5\%), sodass eine Imputation nicht notwendig erschien. Die Prüfung der Daten auf Ausreißer zeigte, dass keine vorliegen. 


\section{Ergebnisse}

\subsection{Dimensionsanalyse}

Der vorliegende Beitrag geht der Frage nach, inwiefern mit einem Onlineinstrument zentrale Facetten des RW-PCKs erfasst werden können. Tabelle 1 gibt dazu eine Antwort, indem die Korrelationen (inkl. Mittelwert und Standardabweichung) der Wissensindikatoren untereinander dargestellt sind. Aus Gründen der Übersichtlichkeit werden nur die statistisch signifikanten Korrelationen gezeigt. Es zeigt sich, dass in Summe wenige und durchwegs niedrige Korrelationen zu beobachten sind, was dazu führt, dass auch im Rahmen einer Faktorenanalyse (Hauptachsenanalyse) die Blöcke jeweils auf eigenen Faktoren laden aber miteinander moderat korreliert sind. Diese Ergebnisse verweisen darauf, dass die einzelnen Facetten des RW-PCK nicht auf ein gemeinsames, latentes Konstrukt zurückgeführt werden können, sondern es sich hierbei eher um voneinander unabhängige Aspekte des Lehrerwissens handelt.

1. Die Quantität und Qualität der Reaktion auf die Schülerfehlvorstellungen (hier im Bereich der Rückstellung) hängt signifikant positiv mit dem Wissen über typische Skills von Schüler/inne/n beim Lösen von Lernaufgaben (hier die Skontoverbuchung) sowie dem Wissen über Kriterien kognitiv aktivierender Lernaufgaben zusammen. Gleichzeitig ist diese Kompetenzfacette negativ mit dem Einsatz unterschiedlicher Darstellungsformen korreliert. Letztere geht allerdings tendenziell positiv mit dem Wissen über Themengebiete des Rechnungswesens, die Schüler/inne/n häufig Lernschwierigkeiten bereiten, einher.

2. Das Lehrerwissen über Skills korreliert positiv mit dem Wissen über Kriterien kognitiv aktivierender Lernaufgaben und tendenziell negativ mit dem Wissen und Anwenden allgemeindidaktischer Modelle.

3. Das Wissen über Kriterien kognitiv aktivierender Lernaufgaben hängt zudem positiv mit der PDK (Rangkomponente und Niveaukomponente) sowie der Anwendung unterschiedlicher Kategorien von Darstellungsformen (traditionell, praxisorientiert, visuell-aktionistisch) im RWUnterricht zusammen.

4. Die PDK (Rang) ist tendenziell positiv mit dem Einsatz von Darstellungsformen und dem Wissen über fachdidaktische Modelle korreliert.

5. Der Einsatz unterschiedlicher Darstellungsformen im RW-Unterricht hängt signifikant positiv mit dem Wissen über und der Anwendung von allgemein- und fachdidaktische(n) Modelle(n) zusammen, welche auch untereinander signifikant korreliert sind. 
Wenn auch nicht ganz konsistent und teils nur schwach bis moderat korreliert, so zeigt sich doch, dass die Indikatoren des fachdidaktischen Lehrerwissens über Schülerkognitionen (Block 1-4), die PDK-Komponenten (Block 5) und die Darstellungsformen sowie Didaktikmodelle (Block 6-8) jeweils wie erwartet untereinander korreliert sind. Die von Berger et al. (2013) angeführte RW-PCK-Unterteilung lässt sich damit mit Bezug auf die Facetten Wissen über Schülerkognitionen und über das Zugänglichmachen von Inhalten auch empirisch nachweisen. Einzig die Indikatoren zum Wissen über das Potential von Lernaufgaben hängen nicht wie erwartet zusammen: Das Wissen über kognitiv aktivierende Lernaufgaben scheint stärker mit dem Wissen über Schülerkognitionen zusammenzuhängen als - wie von uns angenommen mit der PDK bezüglich der Lernaufgaben.

Tab. 1: Signifikante Zusammenhänge zwischen den Facetten des RW-PCKs

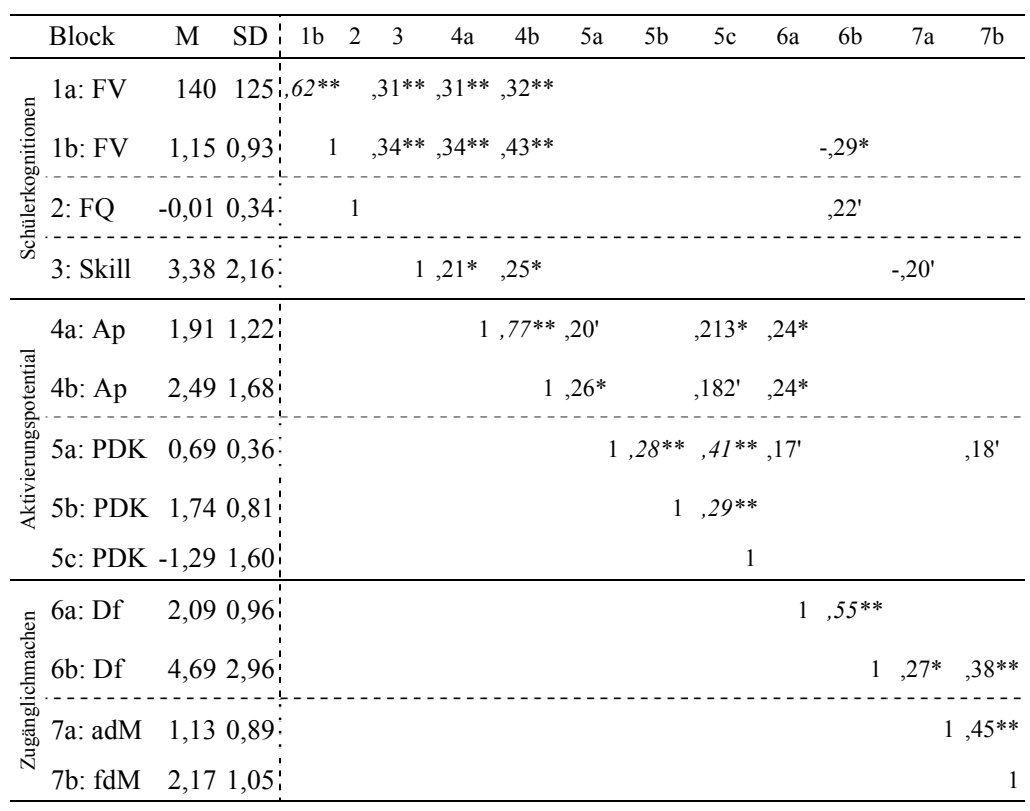

Anmerkungen: M: Mittelwert, SD: Standardabweichung; N = 91; 1 a Fehlvorstellungen I, $1 \mathrm{~b}$ Fehlvorstellungen II, 2 Fehlerquellen, 3 Skills, 4a Aktivierungspotential Lernaufgaben I, $4 b$ Aktivierungspotential Lernaufgaben II, 5 a pädagogischdiagnostische Kompetenz (PDK, Rangkomponente), $5 b$ PDK (Differenzierungskomponente), 5c (PDK Niveaukomponente), 6a Darstellungsformen I, $6 \mathrm{~b}$ Darstellungsformen II, 7 a allgemein-didaktische Modelle, $7 \mathrm{~b}$ fachdidaktische Modelle

Quelle: Eigene Abbildung 


\subsection{Validierungsstudie}

Tabelle 2 enthält Ergebnisse einer Validierungsstudie, im Rahmen derer die Frage gestellt wurde, inwiefern die Wissensindikatoren mit Außenkriterien wie der Selbst- und Fremdeinschätzung zusammenhängen. Interessant ist, dass das Wissen über und die Anwendung von allgemein- und fachdidaktischen Modellen mit beinahe allen Außenkriterien positiv korreliert.

Tab. 2: Validierungsstudie zu den Facetten des $R W$-PCKs

\begin{tabular}{|c|c|c|c|c|c|c|c|c|c|c|}
\hline & $\begin{array}{c}\text { Schwierig- } \\
\text { keit }\end{array}$ & $\begin{array}{l}\text { Trenn- } \\
\text { schärfe }\end{array}$ & $\alpha$ & A & B & C & $\mathrm{D}$ & E & $\mathrm{F}$ & G \\
\hline $\mathrm{M}$ & ! & & & 1,36 & 3,45 & 3,37 & 3,09 & 3,85 & 64,51 & 31,18 \\
\hline SD & & & & 0,66 & 1,33 & 0,70 & 0,69 & 0,56 & 7,72 & 4,49 \\
\hline 1a: FV & .28 & .52 & - & & & & &,$- 213 *$ & &, 302 \\
\hline 1b: FV & .43 & .38 & $.86^{*}$ & & & &,$- 187^{\prime}$ &,$- 258 *$ & & \\
\hline 2: $\mathrm{FQ}$ & .50 & .16 & - & & & & & & & \\
\hline 3: Skill & .48 & .39 & $.84 *$ & & &,$- 223^{*}$ & &,- 204 & & \\
\hline 4a: Ap & .38 & .48 & - & & & & & & ,356 &, 374 \\
\hline 4b: Ap & .36 & .60 & - & & & & & & & \\
\hline 5a: PDK & .85 & .45 & .82 & & & & & & & \\
\hline 5b: PDK & .67 & .23 & .82 & & & & & & & \\
\hline 5c: PDK & .46 & -.16 & .82 & & & & & & &,- 262 \\
\hline 6a: Df & .54 & .43 & .64 & & & & & & & \\
\hline 6b: Df & .34 & .46 & .64 & & ,264* & & & & ,332 & ,279 \\
\hline 7a: adM & .23 & .30 & .75 &, $329 * *$ &, $279 *$ &, $361 * *$ &, $287 * *$ & & ,374 &, 370 \\
\hline $7 \mathrm{~b}: \mathrm{fdM}$ & .29 & .43 & .80 &, $325^{* *}$ &, $323 * *$ &, $281 * *$ & & & ,271 & ,226 \\
\hline
\end{tabular}

Anmerkungen: M: Mittelwert, SD: Standardabweichung; $N=91$ für A-F; $N=20$ für $G$ und $H$; A fachdid. Fortbildungen, B Berufsjahre (Wirtschaft), C Kompetenzorientierung, D Individualisierung, E Diagnosefähigkeit, F kog. Aktivierung (Schülerwahrnehmung), G Verstehen (Schülerwahrnehmung); a Cronbachs Alpha, * Interraterreliabilität

\section{Quelle: Eigene Abbildung}

Lehrerperspektive: Jene Lehrpersonen, die angeben über eine höhere Diagnosefähigkeit zu verfügen, tun dies gemessen an der PDK nicht und schneiden auch bei den Wissensindikatoren Fehlvorstellungen und Skills schlechter $\mathrm{ab}$ als ihre Kolleg/inne/n. Darüber hinaus gilt für Lehrpersonen, die angeben verstärkt kompetenzorientiert bzw. individualisiert zu unterrichten, dass sie eher schlechter bei den Indikatoren Skills bzw. Fehlvorstellungen abschnei- 
den. Des Weiteren ist die Anzahl der Berufsjahre in der Wirtschaft positiv assoziiert mit dem Einsatz unterschiedlicher Darstellungsformen.

Schülerperspektive: Besonders interessant in Hinblick auf die Validität des Onlineinstruments scheint die Gegenüberstellung mit der Schülerperspektive. Tabelle 2 zeigt, dass die Wissensindikatoren Wissen über Fehlvorstellungen, Kriterien kognitiv aktivierender Lernformen, Anwendung unterschiedlicher Darstellungsformen sowie allgemein- und fachdidaktische Modelle sowohl mit dem von den Schüler/inne/n im Unterricht erlebten kognitiven Aktivierungspotential sowie dem Verstehen positiv zusammenhängen. Einzig die PDK sowie das Wissen über Fehlerquellen und Skills sind nicht mit den Schülerurteilen über die Unterrichtsqualität korreliert. Es ist aufgrund der niedrigen Stichprobengröße von 20 Klassen anzumerken, dass die statistischen Signifikanzprüfungen kaum Aussagekraft besitzen. Im Rahmen der Interpretation der Ergebnisse stützen wir uns daher auf die Effektstärken, die allerdings nur gering bis moderat ausfallen.

\section{Diskussion}

Die hier berichtete Studie widmete sich der Frage, inwiefern mittels Onlinebefragung Facetten der RW-PCK erfasst werden können. Die Ergebnisse einer Korrelationsstudie verweisen darauf, dass die Wissensindikatoren untereinander nur schwach bis moderat korreliert sind. Es konnte gezeigt werden, dass die Wissensindikatoren über Schülerkognitionen (1-6), die PDKKomponenten (7-9) und die Darstellungsformen sowie Didaktikmodelle (1013) jeweils untereinander korreliert sind und daher je einen Faktor bilden, was sich auch durch eine hier nicht berichtete Faktorenanalyse tendenziell bestätigen lässt. Zudem hängen die meisten Facetten mit zentralen Außenkriterien wie die Schülereinschätzung zur Unterrichtsqualität zusammen.

Aufgrund dieser eher ernüchternden Ergebnisse muss kritisch gefragt werden, inwiefern das hier vorgestellte Onlineinstrument geeignet ist, um Indikatoren über das RW-PCK von Lehrpersonen zu erfassen.

1. Viele Items, auch wenn sie im Sinne von Performance-Items gestellt wurden, bilden Lehrerurteile ab, die von der tatsächlichen Kompetenz abweichen können.

2. Die PCK-Dimensionen wurden meist nur in Bezug auf ein paar wenige konkrete RW-Inhalte erfasst. So erwies sich lediglich eines von drei Items zur Erfassung des Lehrerwissens über Schülerfehlvorstellungen als angemessen schwierig, um damit weitere Analysen durchzuführen. Damit ist allerdings die inhaltliche Validität nicht länger gegeben, da die inhaltliche Bandbreite des RWs nicht abgedeckt wird. 
3. Die offen formulierten Items verlangen von den Lehrpersonen zudem eine gewisse Anstrengungsbereitschaft beim Beantworten, die mit der eigentlichen Fähigkeit der Lehrperson nur bedingt zusammenhängen muss.

4. Es ist - zumindest für manche Items - fraglich, inwiefern es gelang prognostisch relevante Informationen zu erfassen. So erfasst die Frage nach den angewandten Darstellungsformen möglicherweise eher die Einstellung der Lehrperson gegenüber unterschiedlichen Darstellungsformen und Unterrichtsmethoden, als die fachdidaktische Fähigkeit ein Konzept auf unterschiedlichen Wegen darzustellen.

Diesen Punkten ist allerdings entgegenzuhalten, dass auf theoretischer Basis noch wenig argumentiert ist, weshalb diese Facetten stark miteinander zusammenhängen sollten. So wurde bspw. für die PDK-Komponenten gezeigt, dass diese nicht miteinander korreliert sind (vgl. Spinath 2005). Auch spricht das Wissen über Schülerkognitionen wohl eine andere Kompetenz an (bspw. sich in die Gedankengänge von Schüler/inne/n hineinzuversetzen), als die Fähigkeit RW-Konzepte auf unterschiedliche Weisen darzustellen bzw. zu erläutern (hier ist eine hoch kreative Leistung erforderlich, wenn bspw. Gesetzmäßigkeiten grafisch oder plastisch veranschaulicht werden sollen). Die vorliegende Studie zeigt damit Aspekte auf, die Gegenstand zukünftiger Untersuchungen sein können, nämlich bspw. die Frage, welche Merkmale konkret spezifische Facetten der PCK ausmachen.

Während das Potential der präsentierten Studie in der Breite der erfassten PCK-Facetten sowie in der relativ ökonomischen Erhebung eben dieser liegt (es konnten aus Ressourcengründen keine aufwendigen Assessments mit Lehrpersonen durchgeführt werden), liegen die Grenzen der Studie einerseits in der angesprochenen Validität sowie der Verallgemeinerbarkeit der Ergebnisse. Zur Validität ist allerdings anzumerken, dass das hier angewandte Verfahren des Abgleiches von (Lehrer/innen-)Einschätzungen bzw. Urteilen mit Expertenurteilen bzw. theoretisch fundierten Kriterienkatalogen eine wissenschaftlich bewährte Vorgehensweise darstellt, die sich auch in anderen Kompetenzmessungen, bspw. bei der Erfassung metakognitiver Lernkompetenzen (vgl. Maag Merki, Ramseier \& Karlen 2013), durchgesetzt hat. Zur Repräsentativität ist anzuführen, dass insbesondere jene Analysen, die auf der Teilstichprobe von 20 Lehrer/inne/n bzw. Klassen beruhen, vorsichtig zu interpretieren sind.

Trotz dieser Einschränkungen sind Studien wie die vorliegende von hoher praktischer Relevanz, da sie erste Einblicke in die Struktur und Ausprägung des RW-PCKs von Praktiker/inne/n liefern. Diese Informationen können bei weiteren Forschungen (z.B. Studien zu Effekten des Professionswissens) sowie in fachdidaktischen Lehreraus- und -fortbildungen genutzt werden. 


\section{Literatur}

Baumert, J. \& Kunter, M. (2011). Das mathematikspezifische Wissen von Lehrkräften, kognitive Aktivierung im Unterricht und Lernfortschritte von Schülerinnen und Schülern. In M. Kunter, J. Baumert, W. Blum, U. Klusmann, S. Krauss \& M. Neubrand (Hrsg.), Professionelle Kompetenz von Lehrkräften. Ergebnisse des Forschungsprogramms COACTIV (S. 163-192). Münster: Waxmann.

Baumert, J., Kunter, M., Blum, W., Brunner, M., Voss, T., Jordan, A., Klusmann, U., Krauss, S., Neubrand, M. \& Tsai, Y. (2010). Teachers' Mathematical Knowledge, Cognitive Activation in the Classroom, and Student Progress. American Educational Research Journal, 47(1), 133-80.

Berger, S., Fritsch, S., Seifried, J., Bouley, F., Mindnich, A., Wuttke, E., SchnickVollmer, K. \& Schmitz, B. (2013). Entwicklung eines Testinstruments zur Erfassung des fachlichen und fachdidaktischen Wissens von Studierenden der Wirtschaftspädagogik - Erste Erfahrungen und Befunde. In O. Zlatkin-Troitschanskaia, R. Nickolaus \& K. Beck (Hrsg.), Lehrerbildung auf dem Prüfstand - 2013 Sonderheft. Kompetenzmodellierung und Kompetenzmessung bei Studierenden der Wirtschaftswissenschaften und der Ingenieurwissenschaften (S. 93-107). Landau: Empirische Pädagogik e.V.

Blömeke, S., Bremerich-Vos, A., Kaiser, G., Nold, G., Haudeck, H., Keßler, J.-U. \& Schwippert, K. (Hrsg.). (2013). Professionelle Kompetenzen im Studienverlauf. Weitere Ergebnisse zur Deutsch-, Englisch- und Mathematiklehrerausbildung aus TEDS-LT. Münster: Waxmann.

Blömeke, S., Suhl, U., Kaiser, G., Felbrich, A. \& Schmotz, C. (2010). Lerngelegenheiten und Kompetenzerwerb angehender Mathematiklehrkräfte im internationalen Vergleich. Unterrichtswissenschaft, 38(1), 29-50.

Bühner, M. (2011). Einführung in die Test- und Fragebogenkonstruktion. München: Pearson.

Cauet, E., Borowski, A. \& Fischer, H. E. (2016). Schwierigkeiten bei der Untersuchung von Zusammenhängen zwischen Professionswissen, Unterrichtsqualität und Unterrichtserfolg. Vortrag an der GEBF 2016, Berlin, Online unter: http:// www.gebf2016.de/aktuelles/Symposien_Abstracts_240216.pdf (01.06.2016).

Cauet, E., Liepertz, S., Kirschner, S., Borowski, A. \& Fischer, H. E. (2015). Does it Matter What We Measure? Domain-specific Professional Knowledge of Physics Teachers. Schweizerische Zeitschrift für Bildungswissenschaften, 37(3), 463-480.

Ergönenç, J., Neumann, K. \& Fischer, H. E. (2014). The Impact of Pedagogical Content Knowledge on Cognitive Activation and Student Learning. In H. E. Fischer, P. Labudde, K. Neumann \& J. Viiri (Hrsg.), Quality of Instruction in Physics (S. 145-159). Münster: Waxmann.

Förster, M. \& Zlatkin-Troitschanskaia, O. (2010). Wirtschaftliche Fachkompetenz bei Studierenden mit und ohne Lehramtsperspektive in den Diplom- und Bachelorstudiengängen - Messverfahren und erste Befunde. In K. Beck \& O. ZlatkinTroitschanskaia (Hrsg.), Lehrerbildung auf dem Prüfstand - 2010 - Sonderheft. Lehrerprofessionalität: Was wir wissen und was wir wissen müssen (S. 106-125). Landau: Empirische Pädagogik e.V. 
Fortmüller, R., Kreilinger, L., Rendl, E., Mohr, C. \& Steinbach, L. (2013). Entwicklung der fachdidaktischen Fähigkeit zur Formulierung von Problemstellungen im Fach Rechnungswesen. wissenplus, 5-13/14, 27-32.

Fritsch, S., Berger, S., Seifried, J., Bouley, F., Wuttke, E., Schnick-Vollmer, K. \& Schmitz, B. (2015). The impact of university teacher training on prospective teachers CK and PCK - a comparison between Austria and Germany. Empirical Research in Vocational Education and Training, 7(4), 1-20.

Götzl, M., Jahn, R. W. \& Held, G. (2013). Bleibt alles anders!? Sozialformen, Unterrichtsphasen und echte Lernzeit im kaufmännischen Unterricht. bwp@Berufsund Wirtschaftspädagogik - online, 24, 1-22. Online: http://www.bwpat.de/ausgabe24 /goetzl_etal_bwpat24.pdf (01.06.2016).

Helm, C. (2015). Determinants of competence development in accounting in upper secondary education. Empirical Research in Vocational Education and Training, $7(10), 1-36$.

Helm, C. (in Druck). Berufsbildungsstandards und Kompetenzmodellierung im Fach Rechnungswesen. In Bundesinstitut für Berufsbildung (Hrsg.), Bildungsstandards und Kompetenzorientierung. Herausforderungen und Perspektiven der Bildungs- und Berufsbildungsforschung. Bonn.

Helm, C., Bley, S., George, A. C. \& Pocrnja, M. (2015). Potentiale kognitiver Diagnosemodelle für den berufsbildenden Unterricht. In M. Stock, P. Schlögl, K. Schmid \& D. Moser (Hrsg.), Kompetent wofür? Life-Skills - Beruflichkeit Persönlichkeitsbildung (S. 206-224). Wien: StudienVerlag.

Kelcey, B. (2011). Assessing the Effects of Teachers' Reading Knowledge on Students'Achievement using Multilevel Propensity Score Stratification. Educational Evaluation and Policy Analysis, 33, 458-482.

Klafki, W. (1958). Didaktische Analyse als Kern der Unterrichtsvorbereitung. Die Deutsche Schule, 50(10), 450-471.

Lassnig, F. (2016). 'Duale' oder 'dualistische' Berufsbildung: Gemeinsamkeiten und Unterschiede Österreich-Schweiz-Deutschland, in J. Seifried, S. Seeber \& B. Ziegler (Hrsg.), Jahrbuch der berufs- und wirtschaftspädagogischen Forschung 2016 (S. 23-42), Schriftenreihe der Sektion Berufs- und Wirtschaftspädagogik. Opladen: Barbara Budrich.

Lipowsky, F. (o.J.). Lernen im Beruf - Empirische Befunde zur Wirksamkeit von Lehrerfortbildung. Online: https://www.unikassel.de/einrichtungen/fileadmin/datas/ einrichtungen/zlb/J2010___Lipowsky_Lernen.pdf (21.10.2015).

Lüdtke, O., Trautwein, U., Kunter, M. \& Baumert, J. (2006). Analyse von Lernumwelten: Ansätze zur Bestimmung der Reliabilität und Übereinstimmung von Schülerwahrnehmungen. Zeitschrift für Pädagogische Psychologie, 20, 85-96.

Maag Merki, K., Ramseier, E. \& Karlen, Y. (2013). Reliability and validity analyses of a newly developed test to assess learning strategy knowledge. Journal of cognitive education and psychology, 12(3), 391-408.

Maier, U., Kleinknecht, M., Metz, K. \& Bohl, T. (2010). Ein allgemeindidaktisches Kategoriensystem zur Analyse des kognitiven Potenzials von Aufgaben. Beiträge zur Lehrerbildung, 28(1), 84-96.

Mayer, S. A. (2015). Zusammenhang zwischen ausgewählten Aspekten der Lehrerexpertise und den Schülerleistungen im Rechnungswesenunterricht. Diplomarbeit, Johannes Kepler Universität. Linz. 
Mindnich, A., Berger, S. \& Fritsch, S. (2013). Modellierung des fachlichen und fachdidaktischen Wissens von Lehrkräften im Rechnungswesenunterricht - Überlegungen zur Konstruktion eines Testinstruments. In U. Faßhauer, B. Fürstenau \& E. Wuttke (Hrsg.), Jahrbuch der berufs- und wirtschaftspädagogischen Forschung 2013, (S. 61-72). Opladen: Barbara Budrich.

Neuweg, G. H. (2011). Das Wissen der Wissensvermittler. Problemstellungen, Befunde und Perspektiven der Forschung zum Lehrerwissen. In E. Terhart, H. Bennewitz \& M. Rothland (Hrsg.), Handbuch der Forschung zum Lehrerberuf (S. 451-477). Münster: Waxmann.

Roters, B., König, J., Tachtsoglou, S. \& Nold, G. (2013). Fachdidaktisches Wissen angehender Englischlehrkräfte. Lehrerbildung auf dem Prüfstand, 6(2), 155-177.

Schnick-Vollmer, K., Berger, S., Bouley, F., Fritsch, S., Schmitz, B., Seifried, J. \& Wuttke, E. (2015). Modeling the competencies of prospective business and economics teachers. Professional knowledge in accounting. Zeitschrift für Psychologie, 223(1), 24-30.

Seel, H. (1974). Allgemeine Unterrichtslehre. Wien: ÖBV.

Seifried, J. (Hrsg.). (2004). Fachdidaktische Variationen in einer selbstorganisationsoffenen Lernumgebung. Wiesbaden: Deutscher Universitäts-Verlag.

Seifried, J. (2009): Unterricht aus der Sicht von Handelslehrern. Frankfurt/Main: Peter Lang.

Shulman, L. S. (1987). Knowledge and teaching: Foundations of the new reform. Harvard Educational Review, 57, 1-22.

Spinath, B. (2005). Akkuratheit der Einschätzung von Schülermerkmalen durch Lehrer und das Konstrukt der diagnostischen Kompetenz. Zeitschrift für Pädagogische Psychologie, 19(1/2), 85-95.

van Ophuysen, S. (2010). Professionelle pädagogisch-diagnostische Kompetenz eine theoretische und empirische Annäherung. In N. Berkemeyer, W. Bos, H. G. Holtappels, N. McElvany \& R. Schulz-Zander (Hrsg.), Jahrbuch der Schulentwicklung, 16 (S. 203-234). Weinheim und München: Juventa Verlag.

Weinfurtner, M. R. (2009). Die Bedeutung kaufmännischer Berufspraxis für die Unterrichtsgestaltung von Lehrenden an wirtschaftsberuflichen Schulen in Österreich. Diplomarbeit, Leopold-Franzens-Universität. Innsbruck.

Wuttke, E. \& Seifried, J. (2012). Ansätze der Identifikation typischer Schülerfehler Ergebnisse aus Studien in kaufmännischen Schulen. Unterrichtswissenschaft, 40(2), 174-192. 


\title{
Stellenwert beruflicher Weiterbildung für Betriebe und Beschäftigte im Handwerk
}

\author{
Philipp Struck, Christian Dittmann
}

\section{Ausgangssituation und Problemstellung}

Weiterbildung gewinnt in Handwerksbetrieben zunehmend an Bedeutung. Vor dem Hintergrund eines steigenden Altersdurchschnittes der Beschäftigten wird das Thema vor allem im Kontext langfristiger und demografiesensibler Personalentwicklung immer wichtiger (vgl. Leber, Stegmaier \& Tisch 2013, 1ff.). Trotz der offenkundigen Relevanz von Weiterbildung für eine zukunftsorientierte Personalplanung fällt auf, dass die Weiterbildungsbeteiligung gerade im verarbeitenden Gewerbe des Handwerks unter dem branchenübergreifenden Durchschnitt liegt (vgl. Hartung 2012, 53). Eine Studie des Bundesinstituts für Berufsbildung (BIBB) belegt zudem, dass sich handwerkstypische kleinere und mittlere Betriebe (KMU) seltener für die berufliche Weiterbildung ihrer Beschäftigten engagieren als große Betriebe mit mehreren hundert Beschäftigten (vgl. Behringer \& Schönfeld 2014).

Das Problem der geringen Weiterbildungsbeteiligung von und in KMU wurde durch das Institut für Berufspädagogik und Erwachsenenbildung (IfBE) der Leibniz Universität Hannover 2013 und 2014 im Rahmen von zwei durch die Sozialpartner beauftragten Studien für die Branchen des Metallhandwerks (2013) und der Land- und Baumaschinentechnik (2014) in Niedersachsen erhoben und analysiert (vgl. Dittmann, Meyer, Struck \& Kreutz 2013; Struck, Dittmann \& Meyer 2014).

Die Forschungsfragen beider Studien zielten u.a. auf die Exploration unterschiedlicher Wahrnehmungen der betrieblichen Weiterbildungsaktivität von Geschäftsführungen und Beschäftigten sowie auf deren Einstellungen dazu, die Freizeit der Beschäftigten in Weiterbildungsmaßnahmen einzubeziehen.

In diesem Beitrag werden zentrale Befunde zu diesen Themenfeldern für beide Branchen vergleichend in den Abschnitten 3.1 und 3.2 analysiert. Auf Grundlage der Analyseergebnisse wird eine Skala zur Messung des Stellenwertes (S) der innerbetrieblichen, beruflichen (B) Weiterbildung (W) in KMU hergeleitet: Die SBW-Skala. 


\section{Theoretischer Hintergrund und Stand der Forschung ${ }^{1}$}

In der Untersuchung werden unter „Weiterbildung“ alle Lernprozesse von Beschäftigten verstanden, die ihre berufliche Handlungskompetenz erweitern (siehe u.a. Gillen, Elsholz \& Meyer 2010). Dazu zählen nach Arnold und Müller (2000) Anpassungsfortbildungen, Aufstiegsfortbildungen, Umschulungen, Reaktivierung, Rehabilitation und die Umschulung von Beschäftigten. Unterschiedliche Fachwissenschaften befassen sich mit Weiterbildung im weiteren Sinne, neben der Berufs- und Wirtschaftspädagogik u.a. die Arbeits- und Organisationssoziologie, die Wirtschaftswissenschaften und die (Arbeits-) Psychologie. Gleiches gilt für die betriebliche Weiterbildung, die dominanteste Form der beruflichen Weiterbildung (vgl. Dobischat 1999, 5). Eine wissenschaftlich eindeutige Definition des Weiterbildungsbegriffs ist aufgrund der fachlichen Überschneidungen schwierig (vgl. Gillen et al. 2010, 13).

Verschiedene Studien belegen bundesweit eine unterschiedliche Weiterbildungsaktivität von KMU und Großbetrieben (vgl. u.a. Faulstich 2008; Backes-Gellner 2005; Mesaros, Vanselow \& Weinkopf 2009). Beschäftigte in KMU nehmen demnach deutlich weniger an Weiterbildung teil (vgl. u.a. auch Reinemann 2002, Martin \& Behrends 1999). Dies liegt u.a. daran, dass KMU-Betriebe ihren Beschäftigten deutlich seltener Weiterbildung anbieten (vgl. auch Bellmann \& Leber 2008, 43ff.). Der Anteil von Beschäftigten, die noch nie an einer Weiterbildung teilgenommen haben, ist in KMU überproportional höher als in Großbetrieben (vgl. auch Bellmann, Krekel \& Stegmaier 2010, 47ff.). Entsprechend liegt die Weiterbildungsquote in dem durch KMU-Strukturen geprägten produzierenden Gewerbe bundesweit bei durchschnittlich $29 \%$ und befindet sich damit deutlich unter dem branchenübergreifenden Durchschnitt von 40\% (vgl. Hartung 2012, 53).

Die zeitliche Organisation von Weiterbildung vollzieht sich zudem in einem Spannungsfeld: Weiterbildungen können von wenigen Stunden oder Tagen bis zu mehreren Monaten und Jahren dauern. Mehrheitlich haben Betriebe jedoch keine speziellen Regelungen zur zeitlichen Organisation von Weiterbildung (vgl. Zickert 2007, 22). Häufig setzen sie auf die Bereitschaft der Beschäftigten, an Maßnahmen in ihrer Freizeit teilzunehmen (ebd., 22f.). Dieses Thema findet jedoch nur in Ausnahmefällen Eingang in Tarifverträge und Betriebsvereinbarungen (vgl. Meyer 2007, 251ff.). Insgesamt findet Wieterbildung eher über den Kontext anderer Themen, wie z.B. Qualifizierungsmaßnahmen zur Beschäftigungssicherung, Eingang in Betriebsvereinbarungen (vgl. Busse \& Heidemann 2012).

1 In diesem Kapitel wird der Forschungsstand zusammenfassend dargestellt. Für eine ausführlichere Rezeption empiriegestützter Erkenntnisse über die betriebliche Weiterbildungsaktivität vgl. Dittmann et al. (2013). 
Branchenübergreifende Erhebungen in Niedersachsen bestätigen die bundesweiten Erkenntnisse über Weiterbildung. Betrieblich geförderte Weiterbildung nimmt analog mit der Betriebsgröße zu (vgl. Brandt, Cordes \& Diez 2008, 170f.). Die Mehrzahl der im Rahmen des WepaN („Weiterbildungspanel Niedersachsen") befragten Betriebe in Niedersachsen (2007-2011) gibt zudem an, dass die Qualifizierungsanforderungen an ihre Beschäftigten steigen. 44\% erwarten in diesem Zusammenhang Probleme, ihren Fachkräftebedarf in Zukunft decken zu können. Der geschätzte Qualifizierungsbedarf führt mehrheitlich allerdings nicht zur Steigerung der Weiterbildungsaktivität. Weiterbildung scheint nur in Einzelfällen als eine Strategie gegen den Fachkräftemangel anerkannt zu sein (vgl. Müskens 2011, 3ff.).

Das Niedersächsische Institut für Wirtschaftsforschung stellte 2008 bei $82 \%$ der befragten Betriebe Weiterbildungsbedarf fest. Demgegenüber fördern aber nur $62 \%$ der Betriebe aktiv Weiterbildung. Die Freistellung von Beschäftigten wird von $34 \%$ der nicht weiterbildenden Betriebe als zentrales Argument gegen erhöhte Weiterbildungsanstrengungen angegeben. 24\% der befragten Betriebe geben zudem fehlende finanzielle Mittel für Weiterbildung an (vgl. Brandt et al. 2008, 210ff.).

Aus Arbeitsmarkt- und berufswissenschaftlichen Studien lassen sich teils unterschiedliche Deutungs- und Erklärungsmuster für die unterschiedliche Weiterbildungsaktivität in unterschiedlich großen Betrieben entnehmen. KMU werden u.a. als in der Regel funktional weniger ausdifferenziert als Großbetriebe beschrieben. Da in KMU mehrheitlich keine eigene Bildungsund Personalentwicklungsabteilung existiert, müssen diese bei der Planung und Implementierung von Weiterbildung mit externen Bildungsanbietern und anderen Unternehmen kooperieren. Damit sind Kosten, oft außerhalb der Budgets vieler KMU, verbunden (vgl. u.a. Jütte 2001; Weil 2006). KMU haben oft keinen Betriebsrat, der im Rahmen der Mitbestimmung positive Effekte auf betriebliche Bildung ausüben kann (vgl. Reinemann 2002, 48). Eine Mehrzahl der KMU wird durch die Eigentümerin oder den Eigentümer bzw. durch eine eingestellte Geschäftsführung geführt. Daher hängt die Weiterbildungsaktivität in KMU wesentlich von der Einstellung der Besitzer oder der Geschäftsführung zu Bildungs- und Weiterbildungsfragen ab (vgl. Delbrouck 2000, 19).

\section{Weiterbildung in Handwerksbetrieben. Innerbetriebliche Wahrnehmungen im Branchenvergleich}

Das Problem der geringen Weiterbildungsbeteiligung von KMU wurde zunächst für die Branche des Metallhandwerks in Niedersachsen erhoben (vgl. Dittmann et al. 2013). Dazu wurden eine Fragebogenerhebung und vertiefen- 
de Experteninterviews mit Geschäftsführungen, Beschäftigten und Betriebsratsmitgliedern durchgeführt.

Auf Basis der dabei generierten Befunde wurde 2014 eine Vergleichsstudie in der Branche der Land- und Baumaschinentechnik durchgeführt (vgl. Struck et al. 2014). Das ursprüngliche Forschungsdesign sah ebenfalls eine Befragung mittels Fragebögen sowie leitfadengestützte Experteninterviews vor. Aufgrund der geringen Anzahl an Betrieben in der Branche konnte jedoch die Anonymität der Interviewten nicht gewährleistet werden. Daher wurden die Experteninterviews nicht realisiert.

Die Fragebogenerhebungen ${ }^{2}$ wurde für beide Branchen getrennt für die Gruppe der Mitglieder der Unternehmensleitung und Beschäftigten, sowie für Kleinstbetriebe mit weniger als 30 Beschäftigten und Kleinbetrieben mit 31 bis 250 Beschäftigten ausgewertet. ${ }^{3}$

Die Untersuchungsergebnisse im Metallhandwerk zeigen bereits erhebliche Unterschiede in der inhaltlichen Definition des Weiterbildungsbegriffes zwischen Geschäftsführungen und Beschäftigten. Die unterschiedlichen Interpretationen von Weiterbildung resultieren aus verschiedenen inhaltlichen und organisatorischen Erwartungen. Beschäftigte nehmen z.B. eine über nur wenige Stunden andauernde Schulung oder Unterweisung an einer neuen Maschine im eigenen Betrieb nicht (zwangsläufig) als Weiterbildung wahr. Die Geschäftsführer und Eigentümer verstehen dies durchaus als anpassende Qualifizierungsmaßnahme und damit zugleich als Weiterbildung. Diese unterschiedliche Auslegung des Weiterbildungsbegriffes wurde im Kontext verschiedener Untersuchungsfragen deutlich.

Als Konsequenz aus den unterschiedlichen inhaltlichen Definitionen des Weiterbildungsbegriffes zwischen Unternehmensleitung und Beschäftigten im Metallhandwerk wurde im Fragebogen der Studie in der Land- und Baumaschinentechnik der Begriff der ,beruflichen Weiterbildung“ ausdifferenziert. Auf der ersten Seite des Fragebogens wurde berufliche Weiterbildung als Bildungsangebot, ,das der Aktualisierung, Vertiefung oder Ergänzung der beruflichen Kenntnisse dient“, definiert. Dazu wurden explizit auch „Umschulungen, Aufstiegsfortbildungen, Anpassungsfortbildungen und Unterweisungen (z.B. Herstellerschulungen)“ gezählt.

2 Um Erkenntnisse zu den auf der Basis des Forschungsstandes formulierten Forschungsfragen zu generieren, wurde die „Einstellung“ der Zielgruppen als übergeordneter Begriff festgelegt. Die geschlossenen Fragen wurden fünfstufig gemessen, die Antwortvorgaben der Items reichten von hoher Ablehnung (--), Ablehnung (-), Neutral (0), Zustimmung (+) bis zu hoher Zustimmung (++).

3 Es ist davon auszugehen, dass die beiden Untersuchungsgruppen aus verschiedenen Unternehmen sind. Eine eindeutige Rückführung und Zuordnung der Einzelergebnisse zu den jeweiligen Unternehmen (und Arbeitsgebern) wurde aus Anonymisierungsgründen nicht gewollt. 


\subsection{Wahrnehmungen der betrieblichen Weiterbildungsaktivität}

Im Metallhandwerk schätzen Geschäftsführungen und Beschäftigte die betriebliche Weiterbildungsaktivität sehr unterschiedlich ein. Geschäftsführungen konstatieren eine hohe, Beschäftigte und Betriebsratsmitglieder eine geringe betriebliche Weiterbildungsaktivität. Die betriebliche Weiterbildungsaktivität ist offenbar auch von der Wahrnehmung abhängig, ob und inwiefern eine Weiterbildungsmaßnahme betrieblichen Anforderungen oder individuellen Weiterbildungswünschen der Beschäftigten geschuldet ist. Geschäftsführer/Eigentümer und Betriebsratsmitglieder/Beschäftigte wurden befragt, ob im Betrieb auch individuellen Weiterbildungswünschen der Beschäftigten entsprochen wird. Das Antwortverhalten der Betriebsratsmitglieder/Beschäftigten weicht gegenüber dem der Geschäftsführungen stark ab: Nur 6\% von ihnen stimmen der Aussage zu, wohingegen 28\% die Aussage ablehnen. $42 \%$ geben sogar eine hohe Ablehnung an.

Abb. 1: Einstellungen der Befragten im Metallhandwerk zum Item „Der Betrieb versucht allen individuellen Weiterbildungswünschen der Beschäftigten nachzukommen. "

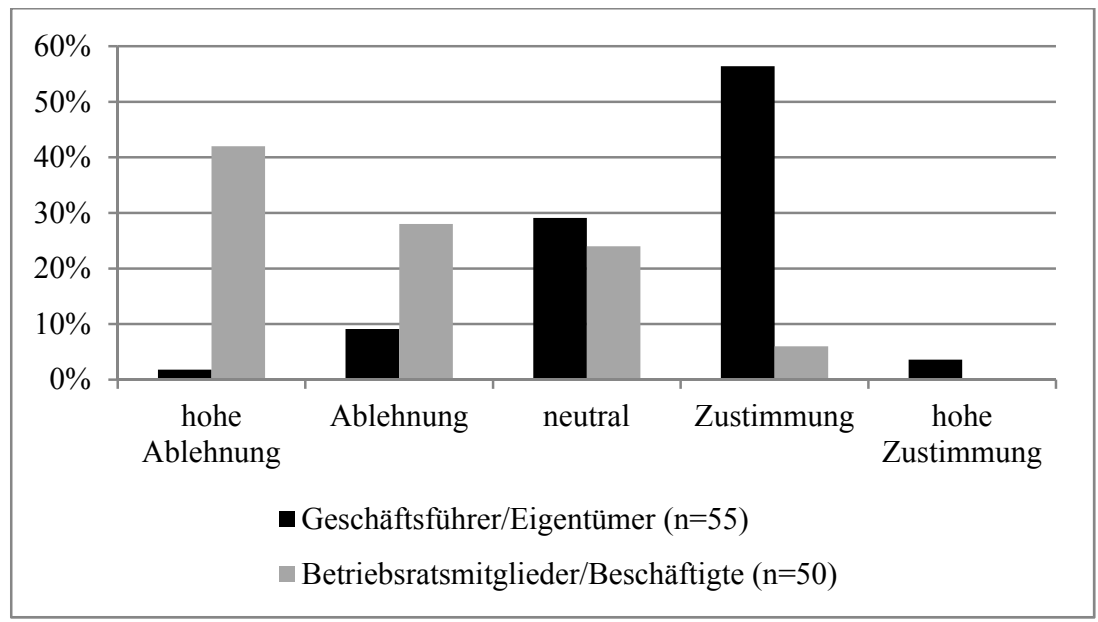

Quelle: Eigene Darstellung.

Demgegenüber weisen die Antworten der Befragten der Land- und Baumaschinentechnik einvernehmlich auf eine hohe Weiterbildungsaktivität der Betriebe hin. Bei getrennter Auswertung nach Betriebsgröße, überrascht die hohe Aktivität der Kleinstbetriebe. 75,1\% der befragten Betriebe mit 31 bis 250 Beschäftigten geben an, dass ihre Beschäftigten mindestens einmal im 
Jahr an Weiterbildungsmaßnahmen teilnehmen. Bei den Betrieben mit 30 und weniger Beschäftigten sind es 93,8\%. Erkenntnisse, die darauf schließen lassen, dass die Weiterbildungsaktivität branchenübergreifend sinkt, desto kleiner der Betrieb ist, bestätigen sich für die Land- und Baumaschinentechnik in Niedersachsen also nicht.

Abb. 2: Einstellungen der Befragten in der Land- und Baumaschinentechnik zum Item ,In unserem Betrieb nehmen mindestens einmal im Jahr Beschäftigte an einer beruflichen Weiterbildung teil. "

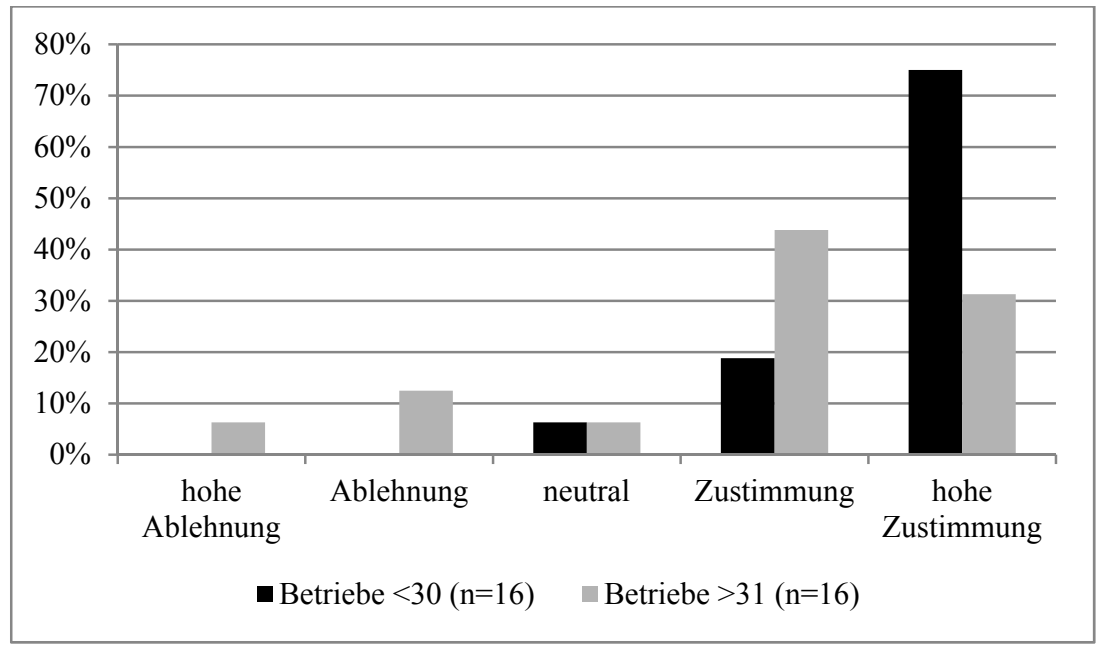

Quelle: Eigene Darstellung.

Einschränkend wurde durch Vertreter der Sozialpartner der Branche darauf hingewiesen, dass die Land- und Baumaschinentechnik sich durch das vergleichsweise hohe Ausmaß betrieblicher Investitionen in Maschinen und Anlagen von anderen Branchen abhebt: Die häufige Anschaffung neuer Maschinen hat einen hohen Schulungsbedarf der Beschäftigten zur Folge. Diese (teilweise für Beschäftigte verpflichtenden) im Zusammenhang mit Neuanschaffungen stehenden Schulungen werden oftmals durch den Hersteller bzw. das vertreibende Unternehmen vorgenommen und sind bereits im Anschaffungspreis der Maschine enthalten. Insofern erklärt sich die hohe Weiterbildungsaktivität der Branche möglicherweise durch die vergleichsweise hohen Investitionen in neue Maschinen und Anlagen. 


\subsection{Weiterbildung in der Freizeit}

Weiterbildung in der Freizeit der Beschäftigten wird weder von den Geschäftsführungen noch den Beschäftigten im Metallhandwerk grundsätzlich abgelehnt. Die befragten Gruppen weichen in ihren diesbezüglichen Antworten jedoch weniger stark ab, als ursprünglich angenommen. $80,7 \%$ der $\mathrm{Ge}-$ schäftsführungen finden es zumutbar, Weiterbildung teilweise in die Freizeit stattfinden $\mathrm{zu}$ lassen. Innerhalb der Gruppe der Betriebsratsmitglieder/Beschäftigten fällt das Ergebnis weniger eindeutig aus. 28,6\% teilen die Auffassung der überwiegenden Mehrheit der Geschäftsführer/Eigentümer, dass Weiterbildung auch in der Freizeit zumutbar ist. Allerdings lehnen dies auch insgesamt 38,8\% ab.

Abb. 3: Einstellungen der Befragten im Metallhandwerk zum Item „Es ist zumutbar, dass Weiterbildung teilweise in der Freizeit stattfindet. "

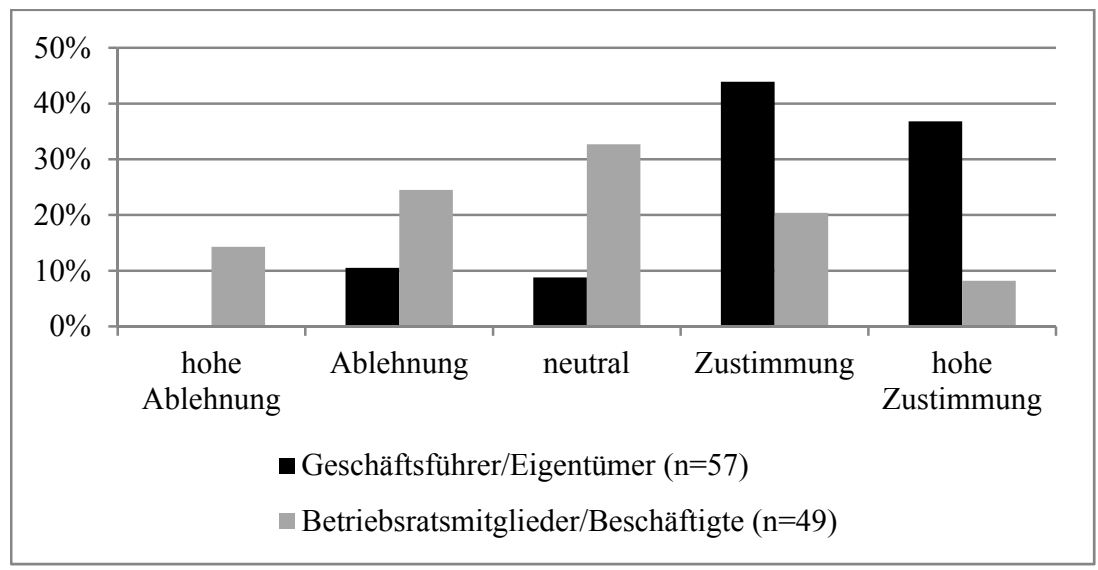

Quelle: Eigene Darstellung.

Die anschließenden Experteninterviews im Metallhandwerk weisen in diesem Zusammenhang darauf hin, dass die Weiterbildungsbereitschaft in der Freizeit der Beschäftigten stark vom persönlichen Nutzen der Maßnahme und dem Betriebsklima abhängig ist.

Bei der getrennten Auswertung des Items in der Land- und Baumaschinentechnik zeigt sich, dass die Antworten der Geschäftsführer die Ergebnisse der Ausgangsuntersuchung für das Metallhandwerk bestätigen. Die Zustimmung der Beschäftigten fällt jedoch mit $43,4 \%$ deutlich höher aus als in der Ausgangsuntersuchung im Metallhandwerk. Nur insgesamt 26\% der befragten Beschäftigten lehnen die Aussage ab. Auffällig ist die hohe Anzahl der 
Beschäftigten, die der Aussage weder zustimmen noch ablehnen (30,4\%). Mit „neutral“ antworten $20 \%$ der Unternehmensleitungen, eine Ablehnung gegenüber der Aussage zeigt aus dieser Gruppe niemand.

Abb. 4: Einstellungen der Befragten in der Land- und Baumaschinentechnik zum Item ,Es ist zumutbar, dass berufliche Weiterbildung teilweise in der Freizeit stattfindet. “

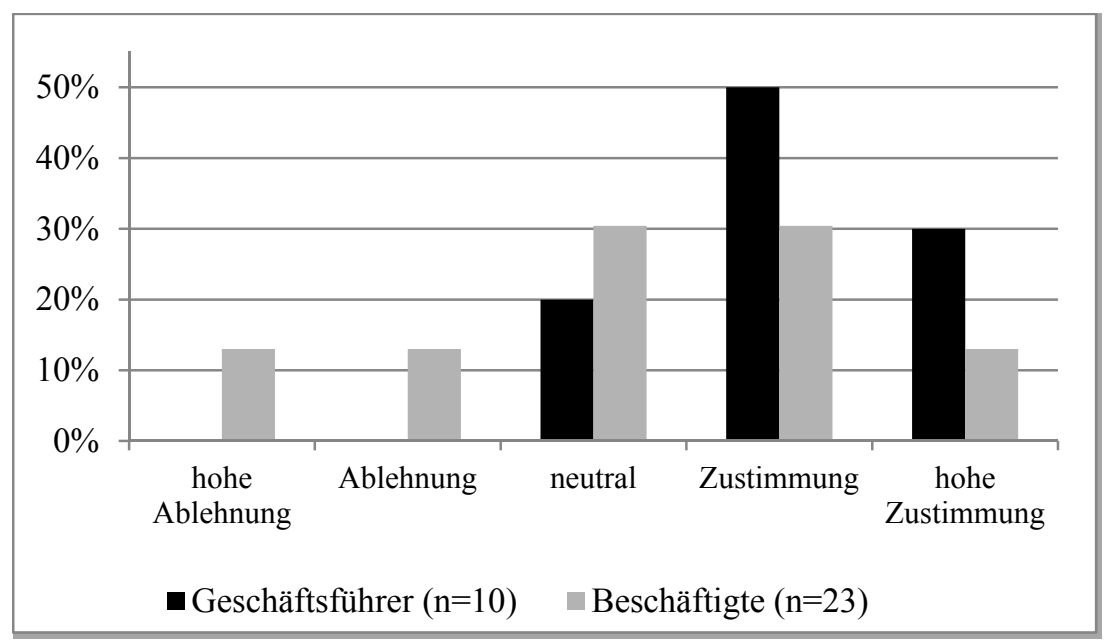

Quelle: Eigene Darstellung.

Zusätzlich wurde das Item in der Branche der Land- und Baumaschinentechnik getrennt für Befragte der Altersgruppen über und unter 41 Jahren ausgewertet. Bei der Auswertung wird sehr deutlich, dass die Gruppe der über 41 Jährigen weitaus eher bereit scheint, an Weiterbildung in der Freizeit teilzunehmen, 88,2\% stimmen der Aussage zu. In der Gruppe der unter 40Jährigen äußert sich ein Großteil (42,9\%) neutral und allein 21,4\% stimmen der Aussage zu. 
Abb. 5: Einstellungen der Befragten in der Land- und Baumaschinentechnik zum Item ,Es ist zumutbar, dass berufliche Weiterbildung teilweise in der Freizeit stattfindet. '

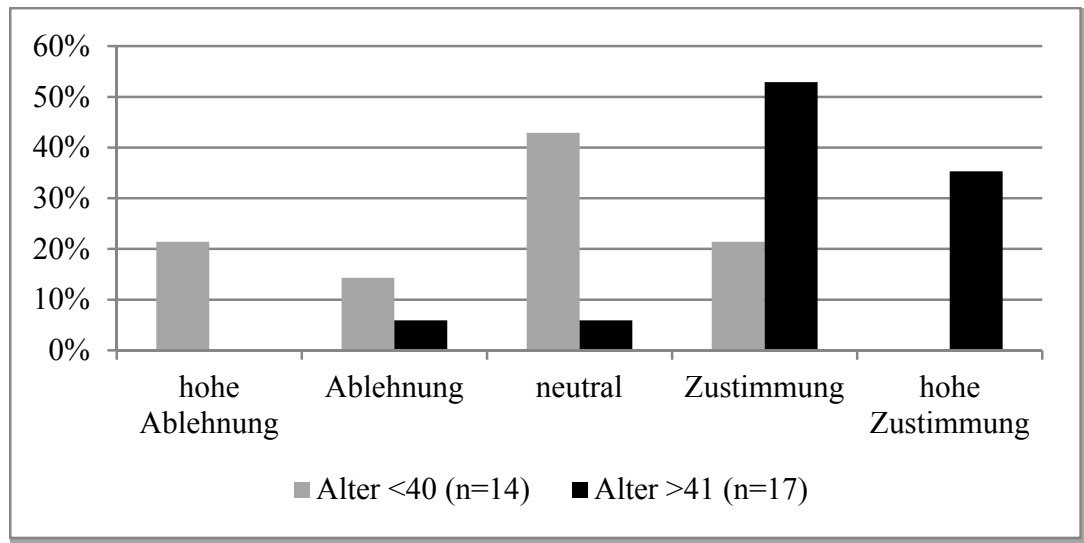

Quelle: Eigene Darstellung.

Möglicherweise sehen die Jüngeren ihre persönliche Work-Life-Balance stärker gefährdet bzw. eine zusätzliche Belastung durch berufliche Weiterbildung in der Freizeit und sind daher weniger bereit diese zur Verfügung zu stellen.

\section{Zusammenführung der Studienergebnisse und Herleitung der SBW-Skala (Stellenwert beruflicher Weiterbildung)}

Um die betriebliche und individuelle Weiterbildungsaktivität betriebs- und branchenspezifisch zu erfassen, bedarf es in der Konsequenz weiterer, vergleichender Forschung: Einerseits sollten die Weiterbildungsbedürfnisse der Betriebe und der Beschäftigten weiterhin getrennt untersucht werden, um Gemeinsamkeiten und Unterschiede gegenüberstellend zu erfassen. Auf Basis dieser Erkenntnisse können dann andererseits Befunde für Betriebe und Segmente bestimmter Beschäftigtengruppen geschaffen werden. Dazu wird auf Basis der Fragebogenerhebung eine Skala entwickelt, welche inhaltlich die Bedeutung bzw. die Relevanz und den Umgang sowie die Kenntnis regionaler Angebote und Möglichkeiten und somit insgesamt den Stellenwert der (beruflichen) Weiterbildung im Betrieb messen soll. Die Items umfassen sowohl Informations- wie Einstellungsfragen. Sie behandeln die betriebliche 
Beteiligung sowie Voraussetzungen, Rahmenbedingungen und Umsetzung von Weiterbildung bzw. die Wahrnehmung des Weiterbildungsstellenwerts im Betrieb und dessen Auseinandersetzung sowie den Grad der eigenen Informiertheit. Die Skala erfragt eine betriebsspezifische Sicht auf das Thema durch Einschätzung der betrieblichen Weiterbildungssituation und damit eine Einschätzung der Bedeutung bzw. des Stellenwerts von Weiterbildung im eigenen Betrieb.

Die Itemformulierungen im Fragebogen (sowie in der Skala) orientieren sich am bestehenden Theorie- und Forschungsstand (siehe Kapitel 2). Konstrukte zur Messung des Stellenwerts beruflicher Weiterbildung liegen in der Literatur nicht vor, weshalb die Items eigens formuliert und entwickelt wurden. Die Skala berücksichtigt nur ausgewählte Items. Diese wurden im Fragebogen nicht im Block getestet, sondern mit anderen Fragen vermischt aufbereitet. Die Fallzahl der ersten Studie erlaubt weiterführende Testungen. Zur Prüfung der Normalverteilung der sechs Items, wurde zunächst die Übereinstimmung von Mittelwert, Modus und Median betrachtet. In den ersten beiden Items weicht der Modalwert mit einem Wert von 4 gegenüber dem Mittelwert und Median von 3 leicht ab. Starke Abweichungen von der Normalverteilung sind insgesamt nicht festzustellen. Die sechs Items werden entsprechend für die Skalenbildung als geeignet betrachtet und herangezogen. Tabelle 1 zeigt die Mittelwerte, Standardabweichung und die ItemTrennschärfen der Items sowie den Konsistenzwert der Skala $\alpha$, ihren Mittelwert und ihre Standardabweichung.

\section{Tab. 1: Skalentest ( $n=101$ Geschäftsführer und Betriebsratsmitglieder)}

\begin{tabular}{lccc}
\hline Item & $\mathrm{M}$ & $\mathrm{SD}$ & $\mathrm{r}_{\mathrm{it}-\mathrm{i}}$ \\
\hline $\begin{array}{l}\text { In unserem Betrieb nehmen mindestens einmal im Jahr } \\
\text { Beschäftigte an einer Weiterbildung teil. }\end{array}$ & 3,08 & 1,32 & 0,59 \\
$\begin{array}{l}\text { Weiterbildungsplanung ist ein fester Bestandteil der betrieb- } \\
\text { lichen Personalplanung. }\end{array}$ & 2,79 & 1,37 & 0,68 \\
\hline $\begin{array}{l}\text { Mir sind die fachlichen Weiterbildungsmöglichkeiten für das } \\
\text { Metallhandwerk bekannt. }\end{array}$ & 3,23 & 1,17 & 0,76 \\
\hline $\begin{array}{l}\text { Mir sind die Weiterbildungsangebote für das Metallhand- } \\
\text { werk in meiner Region bekannt. }\end{array}$ & 3,24 & 1,15 & 0,80 \\
\hline $\begin{array}{l}\text { Die regionalen Weiterbildungsangebote passen zu den An- } \\
\text { forderungen des Betriebs. }\end{array}$ & 3,08 & 0,82 & 0,54 \\
\hline $\begin{array}{l}\text { Der Betrieb nutzt auftragsschwache Zeiten für Weiterbil- } \\
\text { dung. }\end{array}$ & 2,85 & 1,20 & 0,61 \\
\hline$\alpha_{(101)}=0,863$ & 3,04 & 0,91 & \\
\hline
\end{tabular}

M-Mittelwert (Wertebereich: 1-5), SD-Standardabweichung, $r_{i t-i}$-Item-Trennschärfe

Quelle: Eigene Darstellung. 
Die Bildung der Skala erfolgt durch Aufsummieren der einzelnen Items und anschließende Division ihrer Anzahl. Mittelwert, Modus und Median der Skala sind annähernd gleich und deuten ebenso wie das Histogramm, auf eine Normalverteilung der Skala hin. Für den Wertebereich von 1-5 liegt der Skalenmittelwert mit 3,04 nahe dem mathematischen (ideal) Mittelwert von 3,0. Neben der Reliabilitätsanalyse bestätigt auch die Faktorenanalyse (VarimaxMethode) den Zusammenhang der Items. Alle sechs Items laden auf nur einem Faktor bzw. es wurde nur eine Komponente der Ladung ermittelt, bei $60 \%$ aufgeklärter Varianz. Zur weiteren Testung wurde u.a. ein t-Test genutzt, welcher die beiden Untersuchungsgruppen der Studie Geschäftsführer/Eigentümer und Betriebsratsmitglieder/Beschäftigte miteinander vergleicht. Abbildung 6 zeigt, dass erwartungsgemäß die Geschäftsführer bzw. die Eigentümer einen (höchst signifikant) höheren Mittelwert angeben als die Betriebsratsmitglieder bzw. die Beschäftigten.

Abb. 6: Mittelwertvergleich. Wertebereich: 1-5; ***p<.001

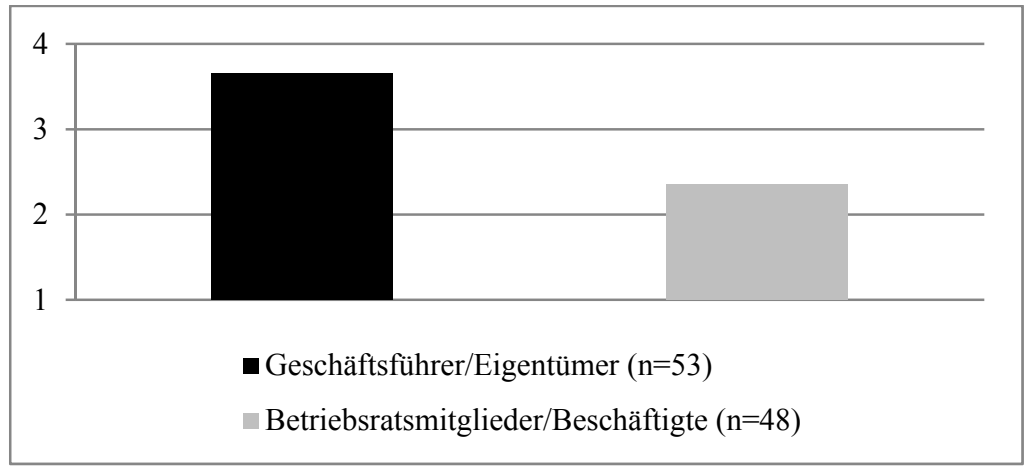

Quelle: Eigene Darstellung.

Dieses Ergebnis lässt sich interpretativ mit den Inhalten der Skala erklären. Die Geschäftsführer und Eigentümer im Metallhandwerk schätzen den Stellenwert bzw. die Bedeutung der Weiterbildung in ihrem eigenen Betrieb höher ein und verfügen gleichzeitig über größere Kenntnisse der regionalen Weiterbildungsangebote als die Betriebsratsmitglieder und Beschäftigten. Möglicherweise ist den Betriebsratsmitgliedern und Beschäftigten die betriebliche Situation teilweise nicht bekannt bzw. sie schätzen sie entsprechend abweichend ein. Geschäftsführer und Eigentümer haben wahrscheinlich einen besseren Überblick über die Aktivitäten und den Umgang mit Weiterbildung im Betrieb. Für die Betriebsratsmitglieder und Beschäftigten bedeutet die Beantwortung der Fragen potenziell eine stärkere Abstrahierung 
und Reflektion. Dies könnte in Verbindung mit geringeren Kenntnissen ein zurückhaltendes Antwortverhalten erklären. Ebenso besteht die Möglichkeit, dass Geschäftsführer und Eigentümer insgesamt die Situation (zu) positiv bzw. positiver als sie tatsächlich ist, einschätzen, während Betriebsratsmitglieder und Beschäftigte die Angebote und Abläufe kritischer beurteilen. Insgesamt entspricht der signifikante Mittelwertunterschied in der Skala plausibel den Erwartungen und Erkenntnissen aus Kapitel 3.

Zur weiterführenden Evaluierung wurde die Skala ebenso in der (vergleichbaren) Studie in der Land- und Baumaschinentechnik eingesetzt. Dabei wurden die Itemformulierungen einerseits an die Branche angepasst sowie zusätzlich die Begrifflichkeit Weiterbildung durch den Zusatz des Wortes „berufliche“ konkretisiert (siehe Tabelle 2). Der Fragenbogenrücklauf entsprach jedoch nicht den Erwartungen, sodass eine ausführliche Skalentestung nicht durchgeführt werden konnte. Für die sehr geringe Stichprobengröße von $\mathrm{n}=31$ konnte die Konsistenz der Skala bestätigt werden $\left(\alpha_{(31)}=.88\right)$. Daraus kann ansatzweise geschlossen werden, dass zum einen die Skala in mehreren Branchen potenziell einsetzbar wäre sowie die Konkretisierung der „,beruflichen Weiterbildung“ Missverständnissen präventiv vorbeugen könnte.

\section{Tab. 2: Die SBW-Skala}

- In unserem Betrieb nehmen mindestens einmal im Jahr Beschäftigte an einer beruflichen Weiterbildung teil.

- Berufliche Weiterbildungsplanung ist ein fester Bestandteil der betrieblichen Personalplanung.

- Mir sind die Möglichkeiten beruflicher Weiterbildung in der Land- und Baumaschinentechnik bekannt.

- Mir sind die Möglichkeiten beruflicher Weiterbildung der Land- und Baumaschinentechnik in meiner Region bekannt.

- Die regionalen beruflichen Weiterbildungsangebote passen zu den Anforderungen des Betriebs.

- Der Betrieb nutzt auftragsschwache Zeiten für berufliche Weiterbildung.

Quelle: Eigene Darstellung.

4 Aufgrund der geringen Stichprobengröße ist eine Reliabilitätsanalyse (eigentlich) unzulässig. Sie dient hier allein dem Versuch der Bestätigung der zuvor ermittelten Erkenntnisse. Dies ist bei der Interpretation dieser Ergebnisse zu berücksichtigen. 
Zusammenfassend lässt sich festhalten, dass die Skala eine hohe Konsistenz aufweist, eine erste Validierung positiv verlief sowie der Einsatz in einer zweiten Branche bzw. Studie (ansatzweise) ebenso erfolgreich durchgeführt werden konnte. Die Skala kann für verschiedene Bereiche und Branchen eingesetzt werden, wenn in den Items 3 und 4 die Formulierung an die jeweilige Branche angepasst wird. Zur weiteren Nutzung werden die Itemformulierungen aus Tabelle 2 empfohlen. Der Zusatz der „beruflichen“ Weiterbildung mindert potenzielle Irritationen und Missverständnisse, konkretisiert die Ergebnisse und erleichtert die Interpretation. Weitere vergleichbare Untersuchungen und weiterführende Skalentestungen (z.B. in weiteren Branchen) sind nötig und wünschenswert.

\section{Relevanz der Ergebnisse für die berufspädagogische Forschung und Praxis}

Die Rahmenbedingungen für Weiterbildung in KMU sind branchenübergreifend in einer Vielzahl von Studien untersucht worden (vgl. u.a. Bellmann \& Leber 2008; Backes-Gellner 2005; Meyer 2004). Diesbezügliche Erkenntnisse bestätigen sich mehrheitlich auch für das von KMU dominierte Metallhandwerk sowie die Land- und Baumaschinentechnik in Niedersachsen. Die in der Untersuchung getrennte Befragung von Geschäftsführungen und Betriebsratsmitgliedern/Beschäftigten stellt jedoch bislang ein Novum dar. Im Ergebnis schätzen die befragten Gruppen beider Branchen die betriebliche Weiterbildungsaktivität teilweise sehr unterschiedlich ein. Auch die Befunde über das potenzielle Konfliktfeld der Weiterbildungspartizipation in der Freizeit, sind nicht eindeutig. Weiterbildung in der Freizeit der Beschäftigten wird in beiden Branchen weder von Beschäftigten noch von Arbeitgebern grundsätzlich abgelehnt.

Insgesamt bedarf es weiterer Forschung, um die in der Untersuchung generierten Erkenntnisse über die betriebliche und individuelle Weiterbildungsaktivität betriebs- und branchenspezifisch zu vertiefen. Dabei ist zu beachten, dass die Untersuchungsergebnisse erhebliche Unterschiede in der inhaltlichen Definition des Weiterbildungsbegriffes zwischen Geschäftsführungen und Beschäftigten zeigen. Die unterschiedlichen Interpretationen von Weiterbildung resultieren aus verschiedenen inhaltlichen und organisatorischen Erwartungen. Betriebsratsmitglieder/Beschäftigte nehmen eine bspw. über nur wenige Stunden dauernde Schulung oder Unterweisung an einer neuen Maschine im eigenen Betrieb nicht zwangsläufig als Weiterbildung wahr, während die Geschäftsführer/Eigentümer dies durchaus als Weiterbildung verstehen und entsprechend angeben. Diese unterschiedliche Auslegung des Weiterbildungsbegriffes wurde im Kontext verschiedener Untersuchungsfragen 
deutlich. Aus diesem Grund empfiehlt es sich, im Vorfeld vertiefender Studien zu untersuchen, was genau die jeweiligen Zielgruppen unter Weiterbildung verstehen und welche Erwartungen mit Weiterbildung verbunden werden. Die praktische Gestaltung der Weiterbildung und die Erhöhung der Weiterbildungsaktivität in Handwerks-KMU können durch geeignete Maßnahmen auf Basis vertiefender Untersuchungen erfolgen. Mittels der im Beitrag vorgestellten $S B W$-Skala kann die betriebliche und individuelle Weiterbildungsaktivität betriebs- und branchenspezifisch erhoben werden. Auch um die betriebliche und individuelle Weiterbildungsaktivität zielgerichtet zu erhöhen, bedarf es weiterer Forschung: Dazu sollten die Weiterbildungsbedürfnisse der Betriebe und der Beschäftigten genauer untersucht werden, um Gemeinsamkeiten und Unterschiede festzustellen. Auf Basis dieser Erkenntnisse könnten anschließend kompatible Angebote geschaffen werden.

\section{Literatur}

Arnold, R. \& Müller, H.-J. (2000). Berufsbildung: Betriebliche Berufsausbildung, berufliche Schulen, Weiterbildung. In H.-H. Krüger \& T. Rauschenbach (Hrsg.), Einführung in die Arbeitsfelder des Bildungs- und Sozialwesens (S.63-91). Opladen: Budrich.

Backes-Gellner, U. (2005). Betriebliche Weiterbildung in kleinen und mittleren Unternehmen. Kosten, Nutzen, Finanzierung beruflicher Weiterbildung. In Bundesinstitut für Berufsbildung (Hrsg.), Ergebnisse der BIBB-Fachtagung vom 2. und 3. Juni 2005 (S.132-153). Bonn.

Behringer, F. \& Schönfeld, G. (2014). Lernen Erwachsener in Deutschland im europäischen Vergleich. In BIBB (Hrsg.): Datenreport zum Berufsbildungsbericht 2014. Informationen und Analysen zur Entwicklung der beruflichen Bildung (S. 381-413). Bielefeld: W. Bertelsmann Verlag.

Bellmann, L., Krekel, E. M. \& Stegmaier, J. (2010). Aus- und Weiterbildung - Komplemente oder Substitute? Zur Bildungsbeteiligung kleinerer und mittlerer Betriebe in Deutschland. Report. Zeitschrift für Weiterbildungsforschung, 33(1), 4154.

Bellmann, L. \& Leber, U. (2008). Weiterbildung für Ältere in KMU. Sozialer Fortschritt, 57(2), 43-48.

Brandt, O., Cordes, A. \& Diez, J. R. (2008). Regionale Beschäftigungsentwicklung der gering und mittel Qualifizierten in Niedersachsen. Bestimmungsfaktoren und Handlungsempfehlungen. Forschungsberichte des Niedersächsischen Instituts für Wirtschaftsforschung, 33, 1-288.

Busse, G. \& Heidemann, W. (2012). Betriebliche Weiterbildung. Reihe Betriebs- und Dienstvereinbarungen. Frankfurt am Main: Bund-Verlag.

Delbrouck, I. (2000). Besonderheiten von KMU. In I. Delbrouck, K. Jutzi \& H. Müller (Hrsg.), Lernen kleine Unternehmen anders? (S.17-19). Rainer Hampp Verlag: München und Mering. 
Dittmann, C., Meyer, R., Struck, P. \& Kreutz, M. (2013). Weiterbildung im Metallhandwerk in Niedersachsen. Rahmenbedingungen und Handlungsfelder. Forschungsbericht im Rahmen des Projektes „Mehr Weiterbildung im Metallhandwerk". Hannover.

Dobischat, R. (1999). Reichweiten und Grenzen des Beitrags beruflicher Weiterbildung zum regionalen Strukturwandel. Netzwerke zwischen Betrieben und überbetrieblichen Weiterbildungsträgern - eine Allianz mit Zukunft? In W. Hendrich \& K. Büchter (Hrsg.), Politikfeld betriebliche Weiterbildung (S. 89-116). München, Mering: Hampp.

Faulstich, P. (2008). Weiterbildung. In: K. S. Cortina, J. Baumert, A. Leschinsky, K. U. Mayer, \& L. Tromer (Hrsg.), Das Bildungswesen der Bundesrepublik Deutschland (S.647-682). Reinbek: Rowohlt.

Gillen, J., Elsholz, U. \& Meyer, R. (2010). Soziale Ungleichheit in der beruflichen und betrieblichen Weiterbildung. Stand der Forschung und Forschungsbedarf. Arbeitspapier 191 der Hans-Böckler-Stiftung. Düsseldorf.

Hartung, S. (2012). Betriebliche Berufsausbildung und Weiterbildung in Deutschland. Nürnberg. Online: https://datenreport.bibb.de/media2013/IAB-Expertise_fuer den Datenreport zum Berufsbildungsbericht 2013.pdf (29.03.2016).

Jütte, W. (2001). Beziehungsgeflechte in lokalen Institutionenlandschaften. Ein theoretischer und empirischer Beitrag zu Netzwerken in der Weiterbildung. In P. Faulstich, G. Wiesner \& J. Wittpoth (Hrsg.), Wissen und Lernen, didaktisches Handeln und Institutionalisierung. Befunde und Perspektiven der Erwachsenenbildungsforschung. Dokumentation der Jahrestagung 2000 der Sektion Erwachsenenbildung der Deutschen Gesellschaft für Erziehungswissenschaft (S.214224). Beiheft zum Report. Bielefeld: Bertelsmann.

Leber, U., Stegmaier, J. \& Tisch, A. (2013). Altersspezifische Personalpolitik. Wie Betriebe auf die Alterung ihrer Belegschaften reagieren. IAB-Kurzbericht 13, 1-8.

Martin, A. \& Behrends, T. (1999). Die Empirische Erforschung des Weiterbildungsverhaltens von Unternehmen. Schriften aus dem Institut für Mittelstandsforschung. Lüneburg: Inst. für Mittelstandsforschung.

Mesaros, L., Vanselow, A. \& Weinkopf, C. (2009). Fachkräftemangel in KMU Ausmaß, Ursachen und Gegenstrategien. Eine Literaturanalyse. Duisburg.

Meyer, R. (2004). Arbeitsprozessorientierte Weiterbildung in Klein- und Mittelbetrieben. Möglichkeiten und Grenzen der Umsetzung am Beispiel des ITWeiterbildungssystems. In P. Dehnbostel \& G. Pätzold (Hrsg.), Innovationen und Tendenzen der betrieblichen Berufsbildung. Beiheft 18 der Zeitschrift für Berufsund Wirtschaftspädagogik (S. 212-221). Stuttgart: Steiner.

Meyer, R. (2007). Primat der Ökonomie? - Arbeitnehmerinteressen in der betrieblichen Weiterbildung. In P. Dehnbostel,U. Elsholz \& J. Gillen (Hrsg.), Kompetenzerwerb in der Arbeit. Perspektiven arbeitnehmerorientierter Weiterbildung (S. 249-262). Berlin: Edition Sigma.

Müskens, W. (2011). Weiterbildung im demographischen Wandel. Ergebnisse des Weiterbildungspanels Niedersachsen (2007-2011). Bericht 9/2011. Online: http:// www.wepan.de/export/sites/default/Dokumente/Bericht-WepaN-2011.pdf, 03.2016).

Reineman, H. (2002). Betriebliche Weiterbildung in mittelständischen Unternehmen. Trierer Schriften zur Mittelstandsökonomie. Münster: Lit Verlag. 
Struck, P., Dittmann, C. \& Meyer, R. (2014). Weiterbildung in der Land- und Baumaschinentechnik in Niedersachen. Forschungsbericht im Rahmen des Projektes „Mehr Weiterbildung im Metallhandwerk“. Hannover.

Weil, M. (2006). Weiterbildungskooperation in KMU. Eine Perspektive für berufsund wirtschaftspädagogische Konzepte. Bern: h.e.p. Verlag.

Zickert, K. (2007). Förderung der beruflichen Qualifizierung durch Weiterbildungsund Arbeitszeitmanagement. Ergebnisse einer Betriebsbefragung. IAB Forschungsbericht 11/2007. Nürnberg. 


\section{Die Beurteilung kaufmännischer Auszubildender in der betrieblichen Praxis - Eine explorative Studie bei Industrie- und Bankkaufleuten}

Andreas Rausch, Julia Warwas, Heike Jost

\section{Ausgangslage und Fragestellung}

In der betrieblichen Berufsausbildung liefern Leistungsbeurteilungen vielfältige Informationen für Übernahme- und Einsatzentscheidungen (z.B. Wenzelmann, Schönfeld, Pfeifer \& Dionisus 2009). Sie bieten aber auch Gelegenheiten, gezielte Rückmeldung zu individuellen Entwicklungsfortschritten und -bedarfen zu geben (vgl. AEVO $\S 3$ Abs 2 Nr. 1, 8). In der einschlägigen Ratgeberliteratur wird die Beurteilung von Auszubildenden daher als ebenso wichtige wie anspruchsvolle Aufgabe des Ausbildungspersonals charakterisiert (z.B. Kiefer \& Knebel 2011; Ruschel 2015). Empirische Beiträge der berufs- und wirtschaftspädagogischen Forschung haben diese Gestaltungsbereiche betrieblicher Lernorte bislang aber weitgehend ausgeklammert und sich stattdessen stark auf schulische Lehr-Lern-Kontexte konzentriert (vgl. Diettrich \& Vonken 2009).

Die betriebsinterne Beurteilung von Auszubildenden stützt sich im Regelfall auf den wiederholten Einsatz schriftlicher Beurteilungsbogen sowie (im Idealfall) auf anschließende, individuelle Feedbackgespräche. Sie orientiert sich damit grundsätzlich an Verfahren, die auch zur Beurteilung der regulär Beschäftigten angewendet werden. Detaillierte Analysen der Verfahrensmerkmale und Instrumente sind jedoch äußerst rar (vgl. Neumann 1987; Klingner, Schuler, Diemand \& Becker 2003) und angesichts aktueller Diskussionen zur Kompetenzdiagnostik in der beruflichen Bildung von großem Interesse.

Die hier berichtete Studie zielt darauf ab, einen vertieften Einblick in die Beurteilungspraxis kaufmännischer Auszubildender zu erhalten. Zum einen soll im Zuge einer Befragung von Personalverantwortlichen in ausbildenden Betrieben näher aufgeschlüsselt werden, wer, wie oft und wann beurteilt. Ferner sollen im Zuge einer Dokumentenanalyse sowohl formale Merkmale der Beurteilungsinstrumente als auch inhaltliche Beurteilungsgegenstände charakterisiert werden. Ergänzend gehen wir der Frage nach, ob eine vergleichende Gegenüberstellung des Banken- und Industriesektors hinsichtlich der genannten Analysekategorien eher generelle oder eher branchenspezifische 
Beurteilungsverfahren für kaufmännische Auszubildende nahe legt. Die zu Vergleichszwecken herangezogenen Ausbildungsberufe Industriekaufmann/frau und Bankkaufmann/-frau gehören beide zu den 'Top Ten' der am stärksten besetzten Ausbildungsberufe (vgl. BIBB 2014), weshalb Beurteilungen dieser Auszubildendengruppen in vielen Ausbildungsstätten routinemäßig und in größerer Anzahl anfallen.

Die Datengewinnung erfolgte zwischen März und Mai 2015 auf dem Wege der schriftlichen Befragung von Industrie- und Finanzunternehmen mithilfe eines von uns entwickelten Fragebogens. Dabei baten wir auch um Zusendung des im jeweiligen Unternehmen eingesetzten Beurteilungsbogens. An der Befragung beteiligten sich 136 Ausbildungsbetriebe; in 84 Fällen wurde der Rückantwort auch ein Beurteilungsbogen beigefügt.

Im Folgenden werden zunächst allgemeine Kennzeichen und Verfahren der Personalbeurteilung, spezifische Funktionen und Anlässe der Beurteilung von Auszubildenden sowie Anforderungsprofile für angehende Bank- und Industriekaufleute erörtert. Nach einer Präzisierung der Forschungsfragen folgen die Eckdaten der empirischen Studie sowie die Ergebnisse quantitativer und qualitativer Auswertungen. Eine Diskussion der Befunde und die Benennung verbleibender Forschungsdesiderate runden den Beitrag ab.

\section{Grundlagen der Leistungsbeurteilung in der betrieblichen Ausbildung}

\subsection{Kennzeichen und gängige Verfahren der Personalbeurteilung}

Die Personalbeurteilung umfasst alle geplanten, formalisierten und kriteriengeleiteten Bewertungen von Organisationsmitgliedern durch hierzu organisationsintern beauftragte Personen auf der Basis sozialer Wahrnehmungsprozesse im Arbeitsalltag (vgl. Domsch \& Gerpott 1992; Jung 2008; Wunderer 2011). Damit weist sie zwar höhere Grade an Systematik und methodischem Anspruch auf als spontane, ereignisgesteuerte Rückmeldungen im Tagesgeschäft (Day-to-day Feedback; vgl. Schmidt 2010), verwendet aber selten aufwändige Verfahren, wie sie in der Management-Diagnostik üblich sind (vgl. Erpenbeck \& von Rosenstiel 2003).

Als etablierte Elemente der Regelbeurteilung identifizieren u. a. Becker (2009) und Dulisch (2011) den Einsatz gebundener, oft standardisierter Einstufungsverfahren, die Durchführung durch eine ranghöhere Führungskraft sowie die Archivierung und Rückmeldung der Ergebnisse an die Beurteilten. Die Einstufung erfolgt meist in vordefinierten, eine Rangfolge indizierenden 
Ziffern- oder Zahlenformaten, um unmittelbare Vergleiche von Mitarbeitenden zu ermöglichen (vgl. Oechsler 2006).

Hinsichtlich der Beurteilungsgegenstände lassen sich idealtypisch (1) eigenschafts- bzw. merkmalsorientierte, (2) verhaltensorientierte und (3) ergebnisorientierte Verfahren abgrenzen, die je spezifische Vorzüge und Nachteile besitzen. So mag die einfache und ökonomische Handhabung eigenschafts- bzw. merkmalsorientierter Verfahren ein wesentlicher Grund für ihre weite Verbreitung sein. Schwierig gestalten sich allerdings die Auswahl aussagekräftiger Eigenschaften, ihre Beschreibung und Skalierung sowie die Spezifikation einer geeigneten Bezugsbasis, um eine urteilsverzerrende Subjektivität (z.B. Strenge- und Mildetendenzen) verschiedener Beurteilender einzudämmen. In der betrieblichen Praxis bedient man sich deshalb gerne aus Standardkatalogen tendenziell domänenübergreifender Beurteilungsskalen (vgl. Becker 2009).

Werden verhaltensorientierte Einstufungsverfahren verwendet, fällt die Wahl meist auf sog. Verhaltensbeobachtungsskalen. Sie erfordern eine Einschätzung der Häufigkeit beobachteter Verhaltensweisen anhand einer typischerweise fünfstufigen Likertskala $(1=$ nie $/ 5=$ immer) (Latham \& Wexley 1977) und erlauben, je nach Verwendungszweck, eine Summierung der dimensionalen Einzelwerte (vgl. Schuler 2004). Die Entwicklung der Beurteilungsdimensionen erfolgt idealerweise durch ein Team von Arbeitsplatzexperten und soll infolge der Fokussierung auf manifestes Verhalten vorschnelle Generalisierungen vermeiden. Wahrnehmungsfehler und Urteilstendenzen der Beurteilenden können aber auch hier nicht ganz ausgeschlossen werden. Zudem ist eine enge Koppelung zwischen Verhalten und Leistung bzw. Erfolg nicht zwingend gewährleistet (vgl. Becker 2009).

Ergebnisorientierte Einstufungsverfahren setzen an diesem Defizit an, indem sie die Erfüllung qualitativer und quantifizierbarer Leistungsparameter bewerten. Mit der strikten Beschränkung auf erzielte Arbeitsergebnisse geht jedoch wiederum eine Vernachlässigung des Arbeitsverhaltens einher (vgl. Oechsler 2006). Darüber hinaus ist es in vielen Arbeitsbereichen nur bedingt möglich, derartige Leistungsparameter vollständig und eindeutig zu definieren.

Realiter kommen zahlreiche Mischformen der hier erläuterten Einstufungsverfahren zum Einsatz. Der unternehmensinternen Entscheidung für oder gegen eine bestimmte Variante können dabei bewusste Abwägungsprozesse seitens der Personalverantwortlichen ebenso wie unhinterfragte Routinen zugrunde liegen (z.B. Schuler 2004). 


\subsection{Funktionen und Anlässe der Beurteilung von Angestellten und Auszubildenden}

Als Instrument des Personalmanagements dient die Beurteilung betrieblicher Mitarbeitender vielfältigen Zwecken. In Anlehnung an Cleveland, Murphy und Williams (1989) bildet sie die Grundlage für interpersonale Entscheidungen (z.B. über Entgeltdifferenzierung, Beförderungen oder Versetzungen innerhalb der Belegschaft) sowie für intrapersonale Entscheidungen (z.B. über individuelle Weiterbildungsbedarfe). Weiterhin trägt sie zur betrieblichen Systemerhaltung durch vorausschauende Personalplanung bei, erfüllt Dokumentationsfunktionen (z.B. zur Rechtfertigung getroffener Personalentscheidungen) wie auch Informations- und Feedbackfunktionen (z.B. in Mitarbeitergesprächen) (vgl. Muck \& Schuler 2004; Schuler 2004).

Dagegen zielt die Beurteilung von Auszubildenden vorrangig auf eine kontinuierliche Bewertung ihres jeweiligen Ausbildungsstandes ab (vgl. Arnold \& Krämer-Stürzl 1999; Ruschel 2015). Damit erfüllt sie in erster Linie intrapersonale Funktionen der Verhaltensanalyse und -steuerung. Konkreter gefasst dient sie der regelmäßigen Überprüfung und gezielten Unterstützung des allgemeinen und fachlichen Arbeits- und Lernverhaltens, aber auch der Motivation des/der einzelnen Auszubildenden. Dies unterstreicht eine Befragung im Bankensektor, in der 143 Ausbildende und 157 Auszubildende übereinstimmend die Förderung von Motivation, Fähigkeiten und Lernbereitschaft, die Früherkennung von Defiziten sowie die Erstellung eines individuellen Stärken-Schwächen-Profils als primäre Funktionen der Auszubildendenbeurteilung benennen (vgl. Klingner et al. 2003). In den Antworten der Probanden tauchen ergänzend und nachrangig Feedback-, Selektions- und Allokationsfunktionen auf. So soll die Auszubildendenbeurteilung bspw. individuelle Beratungen erleichtern sowie betriebliche Übernahme- und Einsatzentscheidungen unterstützen.

Personalbeurteilungen für reguläre Arbeitnehmerinnen und Arbeitnehmer erfolgen sowohl regelmäßig (vor allem zum Zwecke der Entgeltdifferenzierung) als auch anlassbezogen, bspw. bei Ablauf der Probezeit, anstehenden Versetzungen, individuellen Anfragen bzgl. Gehaltserhöhungen, Projektabschlüssen oder Beendigung des Arbeitsverhältnisses (vgl. Jung 2008). Bei Auszubildenden markiert das Ende der Probezeit ebenfalls einen ersten, wichtigen Zeitpunkt der Beurteilung. Weitere Anlässe bieten die Beendigung einzelner Ausbildungsabschnitte und hiermit verknüpfte Abteilungswechsel, Zwischenprüfungen, Entscheidungen über eine Verlängerung oder Verkürzung der Ausbildungszeit oder die Zeugniserstellung zum Abschluss der Ausbildung. Daneben können markante Leistungseinbrüche oder kritische Ereignisse, welche die Erreichung von Ausbildungszielen gefährden, sowie 
ein vorzeitiger Ausbildungsabbruch die Durchführung einer Beurteilung anstoßen (vgl. Arnold \& Krämer-Stürzl 1999; Ruschel 2008).

\subsection{Branchenspezifische Anforderungen und Beurteilungskriterien für Auszubildende}

Um Beurteilungen transparent, gerecht und aussagekräftig zu gestalten, sollten die anzulegenden Kriterien anforderungsanalytisch fundiert sein (vgl. Schuler 2004). Betriebe müssten sich bei der Auszubildendenbeurteilung daher insbesondere an ausbildungsrelevanten Ordnungsmitteln orientieren (vgl. Kiefer \& Knebel 2011). Berufsfeldübergreifend stellt dabei das Bildungsziel der Handlungskompetenz einen zentralen, wenn auch schwer operationalisierbaren, Bezugspunkt dar (vgl. Dehnbostel 2010; Reetz 2006; Seeber \& Nickolaus 2010). Sowohl die Ratgeberliteratur zur Beurteilung von Auszubildenden (z.B. Leischner 2008; Ruschel 2015) als auch eine kultusministerielle Handreichung für die Erarbeitung von Rahmenlehrplänen (vgl. KMK 2011) schlüsselt diesen Kompetenzbegriff in weitere Subdimensionen auf, wobei zumeist Fach-, Selbst-, Sozial- und/oder Methodenkompetenzen unterschieden werden.

Empirische Analysen dokumentieren einen hohen Stellenwert fachlicher Kenntnisse und Fertigkeiten sowohl in den aktuellen Ausbildungsordnungen für kaufmännische Berufe (vgl. Lorig \& Schreiber 2010) als auch in der betrieblichen Beurteilungspraxis der 1980er Jahre (vgl. Neumann 1987). Tabelle 1 listet die von Industrie- und Bankkaufleuten während der Ausbildung zu erwerbenden Kompetenzen entsprechend einer vom Bundesinstitut für Berufsbildung vorgenommenen Sichtung einschlägiger Ordnungsmittel auf. Auch sie unterstreicht die Dominanz fachspezifischer Fähigkeiten und Fertigkeiten, lässt aber zugleich erkennen, dass sozial-kommunikative und vor allem beratende Fähigkeiten im direkten und persönlichen Kundenkontakt bei angehenden Bankkaufleuten einen größeren Raum einnehmen als bei angehenden Industriekaufleuten, die ihrerseits vorrangig in die Planung, Bewertung und Optimierung von diversen innerbetrieblichen Geschäftsprozessen eingebunden sind.

Aufschluss über domänenspezifische Anforderungen geben weiterhin Stellenanzeigen, die zum einen Erwartungen potenzieller Arbeitgeber widerspiegeln (vgl. Frank, Hackel, Helmrich \& Krekel 2014) und zum anderen zur Prüfung und Legitimation getroffener Auswahlentscheidungen dienen (vgl. Jung 2008). Detaillierte Anzeigenanalysen liefern beispielsweise Trost und Weber (2012) für die Zielgruppe der Berufseinsteiger mit abgeschlossener kaufmännischer Ausbildung. 
Tabelle 1. Kompetenzprofile von Bank- und Industriekaufleuten in der Ausbildung im Vergleich

\section{Von Bankkaufleuten zu erwerbende Fähigkeiten und Fertigkeiten}

Beraten von Kunden über Nutzungsmöglichkeiten von Konten, Wahl der Kontoart, verschiedene Zahlungsverkehrsarten

Bearbeiten von Kundenaufträgen im Rahmen der Kontoführung; Abwicklung des nationalen und internationalen Zahlungsverkehrs für Kunden; Beschreiben der Strukturen des internationalen Bankgeschäftes

Beratung über und Vertrieb von Geldanlageprodukten, Versicherungsprodukten und Bausparprodukten

Bearbeiten von Wertpapierorders; Einordnen besonderer Finanzinstrumente; Informieren über steuerliche Auswirkungen bei Anlagen

Mitwirken bei der Bearbeitung von Nachlässen

Beraten von Kunden über Finanzierungsmöglichkeiten

Mitwirken bei der Bearbeitung von Krediten, Einschätzen von Kreditrisiken, Beurteilen von Sicherheiten und Mitwirken bei der Bearbeitung von Sicherungsvereinbarungen

Anwenden von Instrumenten des betrieblichen Rechnungswesens und Controlling; Beurteilen der Kosten und Erlöse einer Kundenbeziehung

Anwenden von Informations- und Kommunikationssystemen; Anwenden von Techniken der Problemlösung sowie der Entscheidungsfindung

Kommunikations- und Kooperationsfähigkeit im Verhältnis zu Kunden, Vorgesetzten und Mitarbeitern

\section{Von Industriekaufleuten zu erwerbende Fähigkeiten und Fertigkeiten}

Verkaufen der Produkte und Dienstleistungen des Unternehmens

Betreiben von Marketingaktivitäten von der Analyse der Marktpotenziale bis zum Kundenservice; Beraten und Betreuen der Kunden

Ermitteln des Bedarfs an Produkten und Dienstleistungen

Einkaufen von Materialien, Produktionsmitteln und Dienstleistungen und Disponieren für die Leistungserstellung oder den Vertrieb

Unterstützen des Prozesses der Auftragserledigung, z.B. in der Leistungserstellung und der Logistik

Bearbeiten von betriebswirtschaftlichen Themen in allen Funktionen des Betriebes (z.B. Finanzierung, Investitionen, Rentabilität, Kostenplanung, -analyse, -verfolgung)

Bearbeiten der Geschäftsvorgänge des Rechnungswesens; Auswerten von Kennzahlen und Statistiken für die Erfolgskontrolle und zur Steuerung betrieblicher Prozesse

Anwenden von Methoden und Instrumenten zur Personalbeschaffung und -auswahl; Planen des Personaleinsatzes und Bearbeiten von Aufgaben der Personalverwaltung

Planen und Organisieren der Arbeitsprozesse inkl. Nutzung fremdsprachlicher Unterlagen

Korrespondieren und Kommunizieren in typischen Situationen in einer Fremdsprache

Arbeiten in Teams und Projekten unter Verwendung aktueller Informations-, Kommunikations- und Medientechniken, Fähigkeiten zur Kommunikation, Kooperation, Moderation, Präsentation, Problemlösung und Entscheidung

Quelle: (BIBB o.J.) 
Das gewünschte Profil von Bankkaufleuten definieren Trost und Weber (2012) - passend zu den in Tabelle 1 umschriebenen Kompetenzfacetten pointiert als „kreative problemorientierte Verkäufer/-in“. Von qualifizierten Bewerberinnen und Bewerbern im Bankensektor wird also in erster Linie hohe Kunden- und Vertriebsorientierung gefordert, dicht gefolgt von Kommunikationsfähigkeiten und -freude (vgl. Frank et al. 2014). Neben einem offenen, freundlichen, sicheren und gepflegten Auftreten wünschen sich die Personalverantwortlichen ausgeprägte „Kooperationsfähigkeit, Begeisterungsfähigkeit, Belastbarkeit, Einfühlungsvermögen, Entscheidungsfreude, Überzeugungskraft, Verhandlungsgeschick, absolute Vertrauenswürdigkeit, Zuverlässigkeit und Akquisitionstärke" (ebd., 46).

Der Stellenanzeigenanalyse von Trost und Weber (2012) zufolge werden hingegen unter Industriekaufleuten im Wesentlichen ,sprachlich und technisch versierte Projektmanager/-innen" gesucht. Profundes betriebswirtschaftliches Wissen sowie eine gewissenhafte Arbeitseinstellung werden hier durchgängig nachgefragt. In nahezu jeder Stellenanzeige werden zudem sprachliche und technische Fähigkeiten genannt. Soziale Fähigkeiten gehören hingegen, verglichen mit dem Bankensektor, zu den seltener eingeforderten Kompetenzfacetten von Industriekaufleuten.

\subsection{Fragestellungen der empirischen Studie}

Unserer explorativen Studie liegen folgende Forschungsfragen zugrunde:

1. Wie lassen sich Beurteilungsverfahren von kaufmännischen Auszubildenden im Bank- und Industriesektor hinsichtlich der beurteilenden Personen, der Häufigkeit und vordringlichen Anlässe der Beurteilung sowie der primären Zwecke der Beurteilung charakterisieren?

2. Welche formalen und inhaltlichen Merkmale weisen die verwendeten Beurteilungsbogen auf?

3. Welche Unterschiede lassen sich zwischen den Beurteilungsverfahren und -instrumenten für angehende Bankkaufleute und angehende Industriekaufleute erkennen? 


\section{Untersuchungsdesign der empirischen Studie}

\subsection{Datengewinnung und Stichprobe}

Zur Beantwortung der aufgeworfenen Fragen wurde eine Querschnittserhebung in Form einer E-Mail-Umfrage im Frühsommer 2015 durchgeführt. Teilnehmende Unternehmen wurden gebeten, einen Fragebogen zur Auszubildendenbeurteilung auszufüllen und, sofern vorhanden, den hierzu verwendeten Beurteilungsbogen einzureichen. Eine vertrauliche Behandlung aller Unterlagen wurde ebenso wie eine anonymisierte Datenauswertung und Veröffentlichung zugesichert.

Durch personalisierte Schreiben an zufällig ausgewählte Ausbildungsbetriebe der interessierenden Branchen, einen werbenden Beitrag im „Forum für AusbilderInnen“ des BIBB (foraus.de) sowie die Unterstützung kooperierender Industrie- und Handelskammern beteiligten sich insgesamt 136 Ausbildungsbetriebe an der Studie. Dabei handelt es sich um 64 Kreditinstitute (im Folgenden „KI“) und 72 Industrieunternehmen (im Folgenden „IU“). Wir erhielten Antworten aus 14 Bundesländern, aber 70 Ausbildungsbetriebe haben ihren Sitz in Bayern, was für den Akquiseerfolg der Industrie- und Handelskammern spricht.

Beide Teilstichproben bestehen überwiegend aus großen Unternehmen mit mehr als 250 Beschäftigten (KI $58 \%$; IU $64 \%$ ) und mittelgroßen Unternehmen zwischen 50 und 249 Beschäftigten (KI $41 \%$; IU $28 \%$ ). In 109 Fällen füllten Personen aus der Ausbildungs- und/oder Personalabteilung den Fragebogen aus. Weitere Rückläufe stammen von Fachkräften, Assistenten/ -innen der Geschäftsführung, Abteilungsleitenden oder der Geschäftsleitung selbst. Insgesamt sendeten 84 Betriebe - darunter $41 \mathrm{KI}$ und $43 \mathrm{IU}$ - neben dem ausgefüllten Fragebogen auch den eingesetzten Beurteilungsbogen ein. Interessierte Unternehmen erhielten im September 2015 eine Ergebnisrückmeldung in Form eines sechsseitigen Flyers.

\subsection{Erhebungsinstrumente und Datenauswertung}

Der fünfseitige Fragebogen beinhaltete geschlossene Angaben zum Unternehmen (z.B. Größe), zu Merkmalen der Beurteilungspraxis (z.B. zuständige Personen) und des verwendeten Beurteilungsbogens (z.B. Herkunft) sowie zur Position des Antwortenden. Ein offenes Antwortformat wurde zur Erfassung von Beurteilungszwecken verwendet. Für die inhaltsanalytische Auswertung wurde ein Kategorienschema deduktiv aus den in Abschnitt 2.2 erläuterten Beurteilungsfunktionen entwickelt. Die Informations- und Feedbackfunktion wurde hierbei in die Kategorie der intrapersonalen Funktionen 
integriert, da eine Trennung kaum möglich war. Tabelle 2 zeigt einen Ausschnitt aus dem entwickelten Kodierleitfaden.

Tab. 2: Ausschnitt aus dem Kodierleitfaden zur Analyse des Zwecks der Auszubildendenbeurteilung

\begin{tabular}{|c|c|c|c|}
\hline Kategorie & Ausprägung & Definition & Ankerbeispiel \\
\hline \multirow[t]{2}{*}{$\begin{array}{l}\text { Inter- } \\
\text { personal }\end{array}$} & $\begin{array}{l}\text { Hilfe zur Übernah- } \\
\text { meentscheidung }\end{array}$ & $\begin{array}{l}\text { Beurteilung dient der Über- } \\
\text { nahme- bzw. Weiterbeschäf- } \\
\text { tigungsentscheidung }\end{array}$ & $\begin{array}{l}\text { „Probezeitübernahme“, } \\
\text { „Übernahme nach } \\
\text { Ausbildung“" }\end{array}$ \\
\hline & $\ldots$ & $\ldots$ & $\ldots$ \\
\hline \multirow[t]{3}{*}{$\begin{array}{l}\text { Intra- } \\
\text { personal }\end{array}$} & $\begin{array}{l}\text { Stärken/Schwächen- } \\
\text { Ermittlung }\end{array}$ & $\begin{array}{l}\text { Beurteilung dient der Ermitt- } \\
\text { lung von Stärken und Schwä- } \\
\text { chen der Auszubildenden }\end{array}$ & $\begin{array}{l}\text { „Stärken-Schwächen- } \\
\text { Analyse“ }\end{array}$ \\
\hline & Förderfunktion & $\begin{array}{l}\text { Beurteilung dient der Förde- } \\
\text { rung des/der Auszubildenden }\end{array}$ & $\begin{array}{l}\text { „Zur Förderung der } \\
\text { fachlichen und persön- } \\
\text { lichen Entwicklung““ }\end{array}$ \\
\hline & $\ldots$ & $\ldots$ & $\ldots$ \\
\hline $\begin{array}{l}\text { System- } \\
\text { erhaltung }\end{array}$ & $\begin{array}{l}\text { Ausbildungsqualität } \\
\ldots\end{array}$ & $\begin{array}{l}\text { Beurteilung dient der Ausbil- } \\
\text { dungsqualität }\end{array}$ & $\begin{array}{l}\text { „zur Verbesserung der } \\
\text { Ausbildung“ }\end{array}$ \\
\hline $\begin{array}{l}\text { Dokumen- } \\
\text { tation }\end{array}$ & $\begin{array}{l}\text { Hinweis für Zeugnis } \\
\ldots\end{array}$ & $\begin{array}{l}\text { Beurteilung ist Grundlage des } \\
\text { Ausbildungszeugnisses }\end{array}$ & $\begin{array}{l}\text { „zur Niederschrift eines } \\
\text { Ausbildungszeugnisses “ }\end{array}$ \\
\hline
\end{tabular}

Quelle: eigene Abbildung

In den Beurteilungsbogen wurden zuerst schlichte formale Merkmale wie etwa Seitenumfang, Itemanzahl, Gesamturteil oder Übernahmeempfehlung ausgezählt. Weiterhin wurden Art, Skalierungen und Operationalisierungshilfen der verwendeten Beurteilungsverfahren klassifiziert (siehe Abschnitt 2.1). Inhaltsbezogene Kategorisierungen erfolgten für die in den Einzelitems erkennbaren Beurteilungskriterien. Die Kategorien wurden in einem gemischt deduktiv-induktiven Vorgehen entwickelt, wobei die in Praxisratgebern und Handreichungen etablierte Unterscheidung von Teildimensionen der beruflichen Handlungskompetenz (Abschnitt 2.3) als erste, einfache Systematisierung diente. Sie wurde einerseits um solche Zusatzkategorien ergänzt, die in den Items der gesichteten Beurteilungsbogen prominent vertreten waren; andererseits wurden die Teildimensionen Fach- und Methodenkompetenz verschmolzen, da die zugehörigen Items selten trennscharf formuliert waren. Tabelle 3 zeigt einen Ausschnitt aus dem zugehörigen Kodierleitfaden.

Die Kodierungen folgten dem Modell der strukturierenden Inhaltsanalyse nach Mayring (2010) und flossen wie auch die geschlossenen Antwortformate des Fragebogens in anschließende deskriptive und inferenzstatistische Auswertungen ein. Für Branchenvergleiche und ausgewählte Merkmalszusammenhänge griffen wir dabei durchgängig auf non-parametrischen Verfah- 
ren zurück. Als Effektstärken werden dementsprechend Cramers V oder Spearmans $r$ für Zusammenhänge und Phi für Differenzen berichtet. Bei mehreren Analysen zu einer Fragestellung wurde eine Alpha-Fehlerkorrektur vorgenommen.

Tabelle 3. Ausschnitt aus dem Kodierleitfaden zur Analyse der Beurteilungskriterien der in den Beurteilungsbogen enthaltenen Items.

\begin{tabular}{|c|c|c|}
\hline Kategorie & Definition & Ankerbeispiel \\
\hline $\begin{array}{l}\text { Fachkompetenz } \\
\text { (+ fachbezogene } \\
\text { Methoden- } \\
\text { kompetenz) }\end{array}$ & $\begin{array}{l}\text { Items, die in Bezug auf fachbe- } \\
\text { zogene Anforderungen im } \\
\text { Arbeitsablauf definiert sind. }\end{array}$ & $\begin{array}{l}\text { Fachwissen, Produktwissen, fachliche } \\
\text { Problem lösen können, mit Arbeitsmit- } \\
\text { teln umgehen können etc. }\end{array}$ \\
\hline $\begin{array}{l}\text { Kognitive } \\
\text { Grund- } \\
\text { fähigkeiten }\end{array}$ & $\begin{array}{l}\text { Items, die auf allgemeine kog- } \\
\text { nitive Fähigkeiten bezogen sind } \\
\text { und keinen unmittelbaren } \\
\text { Fachbezug erkennen lassen. }\end{array}$ & $\begin{array}{l}\text { Auffassungsgabe, Intelligenz, Kon- } \\
\text { zentration, schnell lernen können, } \\
\text { Denkvermögen etc. }\end{array}$ \\
\hline Sozialkompetenz & $\begin{array}{l}\text { Items, die auf gelingende sozia- } \\
\text { le Interaktionen mit internen } \\
\text { oder externen Personen bezo- } \\
\text { gen sind. }\end{array}$ & $\begin{array}{l}\text { Offenheit, Teamfähigkeit, sich in } \\
\text { andere einfühlen können, selbstsicher } \\
\text { auftreten, Bedürfnisse erkennen etc. }\end{array}$ \\
\hline $\begin{array}{l}\text { Arbeitstugenden } \\
\text { \& Arbeitsweisen }\end{array}$ & $\begin{array}{l}\text { Items, die allgemeines Arbeits- } \\
\text { verhalten beschreiben (ohne } \\
\text { Fach- und Interaktionsbezug) }\end{array}$ & $\begin{array}{l}\text { Gewissenhaftigkeit, Pünktlichkeit, } \\
\text { Selbständigkeit, ausdauernd und } \\
\text { beharrlich an einer Sache arbeiten } \\
\text { etc. }\end{array}$ \\
\hline$\ldots$ & $\ldots$ & $\ldots$ \\
\hline
\end{tabular}

Quelle: eigene Abbildung

\section{Befunde der empirischen Studie}

\subsection{Allgemeine Verfahrensmerkmale der Beurteilungspraxis}

83.1\% der Unternehmen gaben an, feste Beurteilungszeitpunkte einzuhalten. Im Laufe einer Ausbildung wird im Durchschnitt neunmal beurteilt; allerdings streuen die Angaben hierzu erheblich $(\mathrm{M}=9.16$; $\mathrm{SD}=5.24$; $\mathrm{Min}=0$; Max =36). Anlassbezogene Beurteilungen (Mehrfachantwort möglich) erfolgen zumeist nach jedem Ausbildungsabschnitt (86.0\%), kurz vor Ende der Probezeit (55.9\%), kurz vor Ausbildungsende (39.0 \%) sowie nach der Zwischenprüfung (18.4\%). Sonstige Anlässe (20.6\%) betreffen bspw. Geschäftsstellen- und Abteilungswechsel oder Projekte der Auszubildenden. Die Beurteilungsergebnisse werden in $99 \%$ der Betriebe den Auszubildenden 
immer zurückgemeldet; in 89.7 \% dienen sie als Grundlage für ein persönliches Gespräch.

$\mathrm{Zu}$ den beurteilenden Personen (Mehrfachantwort möglich) gehören vor allem ausbildende Fachkräfte (84.3\%), Ausbildungsleitende (46.3\%) und Abteilungsleitende (33.6\%). Nur in $57.5 \%$ der Betriebe werden alle beurteilenden Personen für diese Aufgabe geschult; in $21.6 \%$ sind es zumindest einige der zuständigen Personen. In $20.1 \%$ der Betriebe übernehmen durchweg ungeschulte Personen die Auszubildendenbeurteilung.

Die Freitextantworten zu den Beurteilungszwecken erlaubten eine Kodierung von 276 Sinneinheiten. Durchschnittlich wurden in jedem Fragebogen zwei Angaben gemacht. Am häufigsten wurden intrapersonale Funktionen umschrieben (51.6\%), darunter die Identifikation von Stärken und Schwächen, der retrospektive Nachvollzug der bisherigen Entwicklung, die prospektive Identifikation von Entwicklungspotenzialen oder der fördernde Charakter des Feedbacks. Es folgen interpersonale Funktionen (34.5\%) wie etwa Leistungsvergleiche unter den Auszubildenden, Unterstützung von Übernahmeentscheidungen sowie Hinweise auf spätere Einsatzbereiche. Deutlich seltener wurde mit $10.5 \%$ auf die Dokumentationsfunktion verwiesen. Hierunter fallen etwa die Sammlung von Informationen für das Abschlusszeugnis, Transparenz oder die Befolgung von Dokumentationspflichten. Kaum eine Angabe zielt dagegen auf die Funktion der Systemerhaltung (2.9 \%), etwa mit Blick auf eine allgemeine Qualitätssicherung der Ausbildung.

Erwähnenswert ist, dass in größeren Unternehmen eher feste Beurteilungszeitpunkte eingehalten $\left(\chi^{2}=14.3 ; \mathrm{df}=2 ; \mathrm{p}=.001 ; \mathrm{V}=.324\right)$, häufigere Beurteilungen durchgeführt $\left(\mathrm{r}_{\mathrm{S}}=.219 ; \mathrm{p}<.05\right)$ und beurteilende Personen stärker geschult $\left(\mathrm{r}_{\mathrm{S}}=.234 ; \mathrm{p}<.01\right)$ werden als in kleineren Unternehmen. Zudem finden häufiger individuelle Beurteilungsgespräche statt $\left(\mathrm{r}_{\mathrm{S}}=.206 ; \mathrm{p}\right.$ $<.05$ ). Diese branchenübergreifenden Zusammenhänge fallen zwar schwach, aber signifikant positiv aus. Sie können daher vorsichtig als eine mit zunehmender Organisationsgröße einhergehende Professionalisierung der Beurteilungspraxis interpretiert werden, die angesichts stärkerer Personalressourcen und einer höheren Anzahl von Auszubildenden durchaus plausibel scheint.

\subsection{Merkmale der eingesetzten Beurteilungsbogen}

Fast alle Unternehmen (91.9\%) setzen standardisierte Beurteilungsbogen ein. Wo dies der Fall ist, handelt es sich meist um unternehmensweit einheitlich gestaltete Bogen (75.0\%); deutlich seltener gibt es abteilungsspezifische Bogen, die sich in $12.9 \%$ der Betriebe nur geringfügig, in $12.1 \%$ substanziell voneinander unterscheiden. Die Unterschiede liegen nach Angaben der Befragten meist in der Berücksichtigung des Kundenkontakts, in Formalisie- 
rungsvorgaben sowie besonderen fachlichen Anforderungen in den einzelnen Abteilungen. In der überwältigenden Mehrheit der Fälle wurden die Beurteilungsbogen organisationsintern entwickelt, häufig in Kooperation verschiedener Abteilungen sowie in Abstimmung mit dem Betriebsrat und/oder der Jugend- und Auszubildendenvertretung.

Formal betrachtet umfasst ein Bogen durchschnittlich 3.5 Seiten (SD = 2.17; $\operatorname{Min}=1 ; \operatorname{Max}=13)$ und enthält 15 geschlossene Items ( $\mathrm{SD}=9.77$; Min $=3$; $\mathrm{Max}=64)$ sowie 8 offene Items $(\mathrm{SD}=7.44 ; \mathrm{Min}=0$; $\mathrm{Max}=39)$. Die hohen Streuungswerte belegen jedoch eine große Heterogenität der Instrumente. Die Antwortskalen der geschlossenen Items fallen sogar innerhalb einzelner Bogen vielfältig aus, was Mehrfachkodierungen erforderlich machte. In 31 von 84 Beurteilungsbogen sind fünfach gestufte Bewertungen möglich (z.B. von sehr niedrig bis sehr hoch; von 1 bis 5 oder von $A$ bis $E$ ). Dreistufige Antwortskalen sind in 20 Bogen, vierstufige Skalen in 18 Bogen vorhanden. Seltener finden sich Ausprägungen von 1 bis 10 oder Prozentangaben als Bewertungsoptionen.

Des Weiteren wird ersichtlich, dass ausnahmslos Einstufungsverfahren genutzt werden. Dabei dominieren rein eigenschaftsorientierte Einstufungsverfahren (48.8\%), gefolgt von einer Mischung aus eigenschafts- und verhaltensorientiertenVerfahren (32.1\%). Nur selten werden rein verhaltensorientierte Beurteilungen vorgenommen (19\%). Ergebnisorientierte Verfahren waren in der untersuchten Stichprobe nicht zu finden. In $50 \%$ der Items fanden sich Operationalisierungshilfen auf Itemebene. Damit werden den Beurteilenden kurze Texte, Stichworte oder Synonyme geliefert, welche die zu beurteilende Eigenschaft bzw. Verhaltensweise konkret beschreiben. Bei $10.7 \%$ der Items wurden Operationalisierungshilfen auf Ausprägungsebene bereitgestellt. Dabei handelt es sich um Kurztexte oder Stichworte, welche die verfügbaren Ausprägungen der Antwortskala erläutern. Für knapp $40 \%$ der Items wurden keinerlei Operationalisierungshilfen gegeben.

Den Schwerpunkt der Inhaltsanalyse bildete die Kategorisierung der in den Items enthaltenen Beurteilungskriterien. Insgesamt wurden 1.099 Items kodiert. Abbildung 1 zeigt die Beurteilungskriterien in absteigender Reihenfolge ihrer prozentualen Häufigkeit über alle analysierten Items hinweg. Sie verdeutlicht, dass die meisten Items auf die Beurteilung grundlegender Arbeitstugenden und Arbeitsweisen abzielen. Große Beachtung finden außerdem Sozialkompetenzen in zwischenmenschlichen Interaktionen, gefolgt von Fachkompetenzen (inklusive der in den Itemformulierungen kaum separierbaren fachbezogenen Methodenkompetenzen) sowie Interesse bzw. Motivation.

Im Zuge der Kodierung fiel auf, dass $15.5 \%$ der eingesandten Beurteilungsbogen mindestens ein multikriteriales Beurteilungsitem enthielten. Multikriterialität entsteht, wenn zwei oder mehr inhaltlich wenig überlappende 
Kriterien in einer einzigen Aussage beurteilt werden sollen. Das Beurteilungsergebnis ist dann nicht mehr eindeutig interpretierbar. Abbildung 2 zeigt ein solches Beispiel: In der Operationalisierungshilfe auf Itemebene werden sowohl die äußere Erscheinung als auch die sprachliche Ausdrucksfähigkeit zur näheren Beschreibung von „Auftreten” herangezogen, obwohl diese Merkmale im Einzelfall unterschiedlich ausgeprägt sein können.

Abb. 1: Prozentuale Häufigkeiten der vorgefundenen Beurteilungskriterien (100\% entsprechen 1.099 Kodierungen)

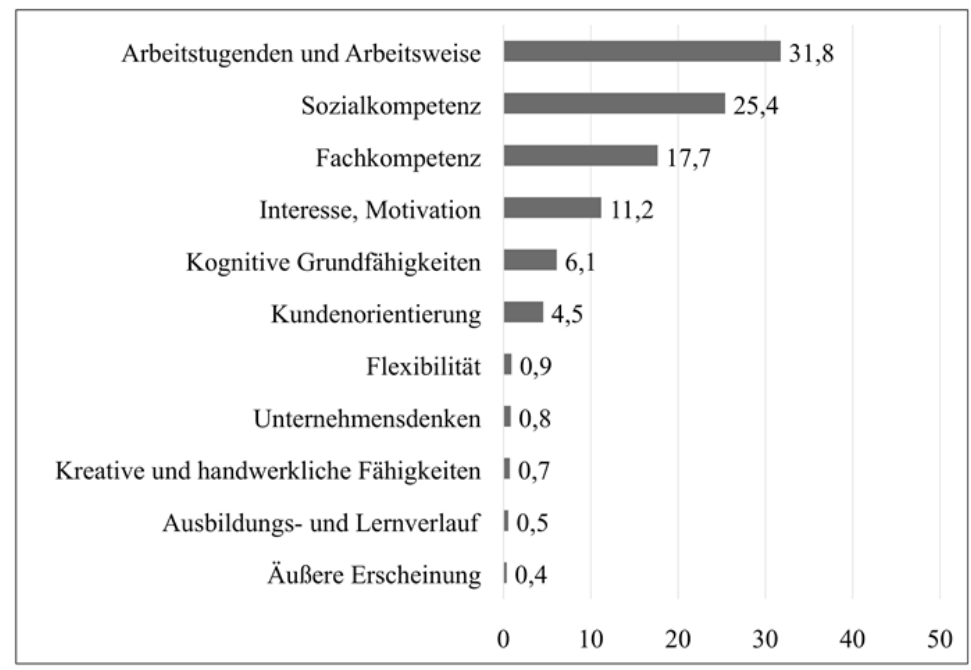

Quelle: eigene Abbildung

Abb. 2: Beispiel eines multikriterial definierten Items

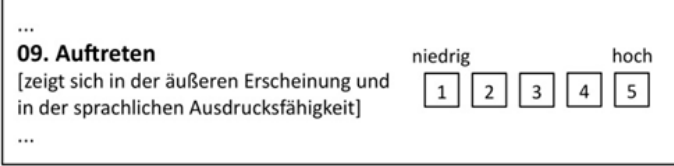

Quelle: eigene Abbildung

Über die Beurteilung konkreter Eigenschaften und Verhaltensweisen hinaus ist in $42.9 \%$ der Beurteilungsbogen ein Einzelitem zur Übernahme- oder Weiterbeschäftigungsempfehlung der Auszubildenden nach Abschluss der Ausbildung enthalten. 


\subsection{Unterschiede zwischen Industrie und Banken}

Alle bisher berichteten Aspekte wurden auf etwaige Branchenunterschiede getestet. Insgesamt ergaben sich hierbei erstaunlich wenige überzufällige Abweichungen zwischen den betrieblichen Beurteilungspraktiken.

Die ausbildenden Finanzdienstleister setzen durchschnittlich häufiger standardisierte Beurteilungsbogen ein als die ausbildenden Industrieunternehmen $\left(\mathrm{KI}=98.4 \%\right.$; IU $=85.9 \% ; \chi^{2}=7.052$, p $_{\text {Fisher }}=.01$, Cramers $\mathrm{V}=$ .229). Zudem sind die Beurteilungsbogen für Bankkaufleute im Durchschnitt etwas länger (Mann-Whitney $\mathrm{U}=577.0, \mathrm{p}=.005$, Phi $=.304$ ) und umfassen mehr dichotome Items als die Bogen der Industrieunternehmen $(\mathrm{U}=644.5, \mathrm{p}$ $=.023$, Phi $=.248)$. Ein bemerkenswerter, wenngleich nicht signifikanter Unterschied zeigt sich in der Schulung der Beurteilenden, die in $31 \%$ der KI, aber nur in $7.9 \%$ der IU nicht erfolgt.

Mit Blick auf die Inhalte der gesichteten Bogen scheint vor allem die „Kundenorientierung“ branchenabhängig unterschiedlich gewichtet zu werden. Dieses Beurteilungskriterium besitzt bei Bankkaufleuten einen deutlich höheren Stellenwert als bei Industriekaufleuten $(\mathrm{U}=302.5, \mathrm{p}=.000$, Phi $=$ .649). Kleinere Differenzen finden sich zudem beim Kriterium „Äußere Erscheinung", das ebenfalls häufiger zur Beurteilung angehender Bankkaufleute herangezogen wird $(\mathrm{U}=795.5, \mathrm{p}=.037, \mathrm{Phi}=.228)$.

\section{Fazit und Ausblick}

Mit der hier berichteten Studie haben wir Gestaltungselemente der betrieblichen Beurteilungspraxis für kaufmännische Auszubildende im Industrie- und Bankensektor analysiert und miteinander verglichen. Insgesamt beteiligten sich 136 Ausbildungsbetriebe an der Studie, von denen 84 auch ihren Beurteilungsbogen zur Verfügung stellten. Angesichts des defizitären Forschungsstands gewährt diese Datenbasis aktuelle und vertiefte Einblicke in betriebliche Beurteilungsverfahren, kann aber keinen Anspruch auf Repräsentativität erheben.

Im Einklang mit den wenigen vorgängigen Studien (z.B. Klingner et al. 2003) betonen die Befragten die intrapersonalen Funktionen der Beurteilung deutlich stärker als die interpersonalen Funktionen. Beurteilungen von Auszubildenden dienen demnach vorrangig der Identifikation individueller Stärken und Schwächen, der Bilanzierung des bisherigen Ausbildungsverlaufs und der Ermittlung von Entwicklungspotenzialen, während z.B. Leistungsvergleiche unter den Auszubildenden nachrangig bewertet werden. Ange- 
sichts der Priorisierung von Diagnose- und Förderzwecken wie auch angesichts der einschlägigen Ausbildungsordnungen und -rahmenpläne muss allerdings verwundern, dass die gesichteten Beurteilungsbogen primär auf eine Bewertung allgemeiner Arbeitstugenden und sozialer Kompetenzen abstellen. Fachkompetenzen der Auszubildenden rangieren hingegen als Beurteilungskriterien deutlich abgeschlagen auf dem dritten Platz und sind zudem in den Itemformulierungen stark mit fachbezogenen Methodenkompetenzen konfundiert. Hierfür bieten sich gleich mehrere Erklärungen an: Möglicherweise vertrauen die Ausbildungsbetriebe darauf, dass positive Ausprägungen genereller, teils persönlichkeitsnaher Merkmale und Verhaltenstendenzen wie Gewissenhaftigkeit den Erwerb fachlicher Kompetenzen stark begünstigen. So betrachtet könnten die Beurteilungsbogen von einer Renaissance domänenübergreifender Schlüsselkompentenzen zeugen, die in der aktuellen wissenschaftlichen Literatur oftmals unter dem Schlagwort der „21st Century Skills” firmieren und berufsfeldunabhängige Facetten der Sozial- und Selbstkompetenz, darunter etwa Lernbereitschaft, Team-, Kommunikations- und Konfliktfähigkeit, zu kritischen Faktoren des beruflichen Erfolges erheben. Denkbar wäre aber auch, dass die Personalverantwortlichen zur Beurteilung der fachlichen Kompetenzen ihrer Auszubildenden die Ergebnisse der Zwischen- und Abschlussprüfungen bei den zuständigen Kammern heranziehen. Da außerdem fachliche Anforderungen teilweise zwischen den durchlaufenen Abteilungen differieren, könnten manche Ausbildungsbetriebe auch den Zusatzaufwand scheuen, der mit dem Entwurf und der Pflege abteilungsspezifisch zugeschnittener Beurteilungsbogen verbunden ist. Zumindest gibt nur knapp ein Siebtel der Befragten an, derart inhaltlich ausdifferenzierte Bogen einzusetzen.

Auf jeden Fall stimmt die Randstellung fachlicher und fachmethodischer Kompetenzen in den von uns gesichteten Beurteilungsbogen (knapp $18 \%$ der kodierten Items) skeptisch, ob pädagogisch wertvolle Lernpotenziale regelmäßiger Beurteilungen im Ausbildungsverlauf mithilfe der hierzu eingesetzten Instrumente voll auszuschöpft werden können. $\mathrm{Zu}$ dieser skeptischen Einschätzung trägt auch der Befund bei, dass die Items in den Beurteilungsbogen häufiger eigenschafts- als verhaltensorientiert formuliert sind, infolge fehlender Operationalisierungshilfen teilweise große Interpretationsspielräume eröffnen und damit Wahrnehmungsfehler wie den Halo-Effekt verstärken können. Das Beurteilungskriterium ,Motivation', verbunden mit einer unkommentierten Einstufungsskala von $1=$ sehr gering bis $5=$ sehr hoch, mag hierfür ein extremes Beispiel abgeben.

Offen bleibt in der vorgenommenen Dokumentenanalyse jedoch, ob in den nach Unternehmensangaben konsequent realisierten FeedbackGesprächen schriftliche Beurteilungskriterien detailliert erläutert und zum 
Anlass genommen werden, einem/einer Auszubildenden konkrete Schritte zur Verbesserung seines/ihres allgemeinen Lern- und Arbeitsverhaltens wie auch seiner/ihrer fachspezifischen Fähigkeiten und Fertigkeiten aufzuzeigen. In Folgestudien scheint es daher lohnend, die Motivations- und Lernpotenziale schriftlicher Beurteilungsergebnisse und mündlicher Besprechungen auch aus der Sicht von Auszubildenden zu beleuchten. Deren Einschätzungen könnten nicht nur motivierende und lernförderliche Gestaltungselemente der betrieblichen Beurteilungspraxis schärfer konturieren, sondern auch gewinnbringend in mehrebenenanalytischen Auswertungen mit organisationalen Merkmalen der Ausbildungsbetriebe verknüpft werden.

Als organisationale Determinanten der Beurteilungspraxis könnten in weiterführenden Studien neben der von uns betrachteten Unternehmensgröße bspw. auch Markt- und Kundensegmente berücksichtigt werden. Die vorliegenden Analysen im Banken- und Industriesektor legen jedenfalls nahe, dass Beurteilungen kaufmännischer Auszubildender in großen Betrieben regelmäBiger stattfinden, häufiger von geschulten Personen durchgeführt werden und öfter um individualisierte Rückmeldungen ergänzt werden als in kleinen Betrieben. Bedeutsame branchenabhängige Differenzen beschränken sich hingegen auf die Verwendung der beiden Beurteilungskriterien Kundenorientierung und Äußere Erscheinung, die für angehende Bankkaufleute häufiger als für angehende Industriekaufleute herangezogen werden. Sie spiegeln damit nur ansatzweise die unterschiedlichen Kompetenzfacetten wider, die aus den gesichteten Stellenanzeigen- und Anforderungsanalysen beider Ausbildungsberufe hervorgehen.

Abschließend ist festzuhalten, dass Beurteilungen von Auszubildenden trotz der von den befragten Personalverantwortlichen betonten Diagnose- und Förderfunktionen mit Blick auf eine mögliche Weiterbeschäftigung oftmals auch „high stakes tests“ darstellen: In knapp der Hälfte der ausgewerteten Beurteilungsbogen wurde eine diesbezügliche Empfehlung explizit erbeten. Berücksichtigt man ferner die Tatsache, dass in den untersuchten Unternehmen überwiegend ausbildende Fachkräfte Beurteilungen vornehmen, die nicht zwangsläufig für diese Aufgabe geschult sind, verdient die Qualität der verwendeten Beurteilungsinstrumente auch in künftigen Untersuchungen besondere Aufmerksamkeit. Verhaltensorientierte Itemformulierungen mit Operationalisierungshilfen sowie eine konsequente Anpassung der Beurteilungskriterien an berufsfeld- wie auch abteilungsspezifische Anforderungen können dazu beitragen, Beurteilungen von Auszubildenden verlässlich und vergleichbar sowie inhaltlich aussagekräftig und entwicklungsförderlich zu gestalten. 


\section{Literatur}

Arnold, R. \& Krämer-Stürzl, A. (1999). Berufs- und Arbeitspädagogik. Leitfaden der Ausbildungspraxis in Produktions- und Dienstleistungsberufen (2. Auflage). Berlin: Cornelsen.

Ausbilder-Eignungsverordnung (AEVO) vom 21. Januar 2009, Bundesgesetzblatt 2009 Teil I Nr. 5, Bonn, 88-92. Online: https://www.bibb.de/dokumente/pdf/ ausbilder_eignungsverordnung.pdf(12.04.2016).

Becker, F. G. (2009). Grundlagen betrieblicher Leistungsbeurteilung. Leistungsverständnis und -prinzip, Beurteilungsproblematik und Verfahrensprobleme (5. Auflage). Stuttgart: Schäffer-Poeschel.

Bundesinstitut für Berufsbildung (BIBB) (2014). Rangliste 2014 der Ausbildungsberufe nach Neuabschlüssen in Deutschland. Online: https://www.bibb.de/dokumente/pdf/naa309_2014_tab67_0bund.pdf(12.04.2016).

Bundesinstitut für Berufsbildung (BIBB) (o.J.). Informationen zu Aus- und Fortbildungsberufen. Online: https://www.bibb.de/de/40.php (09.04.2016)

Cleveland, J. N., Murphy, K. R. \& Williams, R. E. (1989). Multiple Uses of Performance Appraisal: Prevalence and Correlates. Journal of Applied Psychology, 74(1), 130-135.

Dehnbostel, P. (2010). Betriebliche Bildungsarbeit. Kompetenzbasierte Aus- und Weiterbildung im Betrieb. Baltmannsweiler: Schneider Verlag Hohengehren.

Diettrich, A. \& Vonken, M. (2009). Zum Stellenwert der betrieblichen Aus- und Weiterbildung in der Berufs- und Wirtschaftspädagogik. bwp@Berufs- und Wirtschaftspädagogik - online, 16, 1-20. Online: www.bwpat.de/ausgabe16/ diettrich_vonken_bwpat16.pdf (07.03.2016).

Domsch, M. \& Gerpott, T. J. (1992). Personalbeurteilung. In E. Gaugler \& W. Weber (Hrsg.), Handwörterbuch des Personalwesens. Enzyklopädie der Betriebswirtschaftslehre, Band V (2. Auflage), (S. 1631-1641). Stuttgart: Poeschel.

Dulisch, F. (2011). Personalbeurteilung. In T. Fischer (Hrsg.), Personalmanagement. Schriftenreihe: Moderne Verwaltung in der Lehre (S. 185-203). Frankfurt: Verlag für Verwaltungswissenschaft.

Erpenbeck, J. \& von Rosenstiel, L. (2003). Einführung. In J. Erpenbeck \& L. von Rosenstiel (Hrsg.), Handbuch Kompetenzmessung. Erkennen, verstehen und bewerten von Kompetenzen in der betrieblichen, pädagogischen und psychologischen Praxis (S. IX-XL). Stuttgart: Schäffer-Poeschel.

Frank, I., Hackel, M., Helmrich, R. \& Krekel, E. M. (2014). Entwicklungen und Perspektiven von Qualifikation und Beschäftigung im Bankensektor. Online: https://www.bibb.de/veroeffentlichungen/de/publication/show/id/7363 (12.04.20 16).

Jung, H. (2008). Personalwirtschaft (8. Auflage). München: Oldenbourg Wissenschaftsverlag.

Kiefer, B.-U. \& Knebel, H. (2011). Taschenbuch Personalbeurteilung. Feedback in Organisationen. Arbeitshefte Personalpraxis. Band 1 (12., überarbeitete und erweiterte Auflage). Hamburg: Windmühle.

Klingner, Y., Schuler, H., Diemand, A. \& Becker, K. (2003). Konzeption und Prüfung eines multimodalen Systems der Leistungsbeurteilung für Auszubildende. Zeitschrift für Personalpsychologie, 2(3), 133-144. 
KMK (2011): Handreichung für die Erarbeitung von Rahmenlehrplänen der Kultusministerkonferenz für den berufsbezogenen Unterricht in der Berufsschule und ihre Abstimmung mit Ausbildungsordnungen des Bundes für anerkannte Ausbildungsberufe. Online: www.kmk.org/fileadmin/Dateien/veroeffentlichungen_beschluesse/2011/2011_09_23_GEP-Handreichung.pdf (12.04.2016).

Latham, G. P. \& Wexley, K. N. (1977). Behavioral Observation Scales for Performance Appraisal Purposes. Personnel Psychology, 30(2), 255-268.

Leischner, D. (2008). Berufs- und Arbeitspädagogik. Fachwissen und Prüfungsvorbereitung zur AEVO für Ausbilder. München: VLB.

Lorig, B. \& Schreiber, D. (2010). Kompetenzorientierung in Ausbildungsordnungen. In D. Münk \& A. Schelten (Hrsg.), Kompetenzermittlung für die Berufsbildung. Verfahren, Probleme und Perspektiven im nationalen, europäischen und internationalen Raum (S. 119-134). Bielefeld: Bertelsmann.

Mayring, P. (2010). Qualitative Inhaltsanalyse. Grundlagen und Techniken (11. Auflage). Weinheim: Beltz.

Muck, P. M. \& Schuler, H. (2004). Beurteilungsgespräch, Zielsetzung und Feedback. In H. Schuler (Hrsg.), Beurteilung und Förderung beruflicher Leistung. Schriftenreihe Wirtschaftspsychologie (2. Auflage) (S. 255-289). Göttingen: Hogrefe.

Neumann, R. (1987). Art und Umfang der betrieblichen Beurteilung von Auszubildenden. Eine empirische Untersuchung im Bezirk der Industrie- und Handelskammer zu Bochum. Universität Bochum.

Oechsler, W. A. (2006). Personal und Arbeit. Grundlagen des Human Resource Management und der Arbeitgeber-Arbeitnehmer-Beziehungen (8. Auflage). München: Oldenbourg.

Reetz, L. (2006). Kompetenz. In F.-J. Kaiser \& G. Pätzold (Hrsg.), Wörterbuch Berufs- und Wirtschaftspädagogik (2. Auflage) (S. 305-307). Bad Heilbrunn: Klinkhardt.

Ruschel, A. (2008). Arbeits- und Berufspädagogik für Ausbilder in Handlungsfeldern (2., völlig neue Auflage). Ludwigshafen (Rhein): Kiehl.

Ruschel, A. (2015). Beurteilung von Auszubildenden. Online: http://www.adalbertruschel.de/downloades/beurteilung\%20von\%20auszubildenden.pdf (09.11.2015).

Schmidt, K.-H. (2010). Leistungsbeurteilung, Leistungsfeedback und Feedbackwirkungen. In U. Kleinbeck \& K.-H. Schmidt (Hrsg.), Arbeitspsychologie (S. 139176). Göttingen: Hogrefe.

Schuler, H. (2004). Leistungsbeurteilung - Gegenstand, Funktionen, Formen. In H. Schuler (Hrsg.), Beurteilung und Förderung beruflicher Leistung. Schriftenreihe Wirtschaftspsychologie (2. Auflage) (S. 1-23). Göttingen: Hogrefe.

Seeber, S. \& Nickolaus, R. (2010). Kompetenzmessung in der beruflichen Bildung. Berufsbildung in Wissenschaft und Praxis, 2010(2), 10-13.

Trost, S. \& Weber, S. (2012). Fähigkeitsanforderungen an kaufmännische Fachkräfte - Eine kompetenzbasierte Analyse von Stellenanzeigen mittels O*NET. Zeitschrift für Berufs- und Wirtschaftspädagogik, 108(2), 217-242.

Wenzelmann, F., Schönfeld, G., Pfeifer, H. \& Dionisius, R. (2009). Betriebliche Berufsausbildung: Eine lohnende Investition für die Betriebe. Ergebnisse der BIBB-Kosten- und Nutzenerhebung 2007. BIBB Report, 3(8), 1-11.

Wunderer, R. (2011). Führung und Zusammenarbeit. Eine unternehmerische Führungslehre (9. Auflage). Köln: Luchterhand. 


\section{Lesbarkeit der Branchen - Notizen zur Ordnungssemantik des Gewerbes in Realienbüchern des 19. Jahrhunderts ${ }^{1}$}

Frank Ragutt

\section{Einleitung}

Dem Schulbuch kam unter den Schulmännern des 19. Jahrhunderts im Prozess der Institutionalisierung der Bildung eine große Aufmerksamkeit zu. Galt es doch als Garant für die bewahrende Weiterleitung des erarbeiteten Wissens an die nächste Generation. Spätestens seit Johann Amos Comenius' Orbis sensualium pictus (zu Deutsch: Die lesbare Welt) kam Realienbüchern die Aufgabe zu, unter der zu bildenden Generation ein Ordnungsbewusstsein über die natürlichen und künstlichen Dinge der Welt zu erzeugen. Die Umsetzung der Aufgabe erfolgte nicht widerspruchsfrei. Das, was dabei jeweils als ein ,gutes“ bzw. ein ,schlechtes“ Weltbewusstsein definiert wurde, unterlag dem allgemein-epochalen Einfluss ebenso wie den pädagogischen Weltanschauungen (z.B. Heinze 2013; Siegfried 1979). In der Bildungshistoriographie ist seit zwei Jahrzehnten ein wachsendes Interesse an der Schulbuchanalyse zu beobachten (vgl. Wiater 2003; Tröhler \& Oelkers 2005). Historische Schulbuchanalyse - zumeist eingebunden in die pädagogische Schulbuchforschung insgesamt - nähert sich den Quellen im Rahmen thematischer Perspektiven wie Familie (vgl. Matthes \& Heinze 2006), Freund- und Feindbilder (vgl. Durovic \& Matthes 2010) oder dem Bild (vgl. Heinze 2010) an. Die Analysen beziehen sich allerdings in der Regel auf die Materialien der allgemeinbildenden Schulen. In der Berufsbildungsforschung ist die historische Schulbuchanalyse eine Unbekannte.

Der Beitrag wird unter dem Titel „Lesbarkeit der Branchen“ eine erste thesenhafte, keinesfalls Vollständigkeit deklarierende, thematische Bearbeitung historischer Schulbuchforschung für die berufliche Bildung geben. Im Fokus stehen Realienbücher des 19. Jahrhunderts. Das Thema ist der Ordnungsdiskurs des Gewerbes, des Vorläufers des heute geläufigeren Begriffs der Branche. Prominenter Ausgangspunkt in der Geschichte der Realienbücher ist die erwähnte Lesefibel von Comenius, die Anfang der zweiten Hälfte

1 Für die Bearbeitung des vorliegenden Beitrags habe ich vor allem Dr. Robert Maier vom Braunschweiger Georg-Eckert-Institut für internationale Schulbuchforschung zu danken, der mir mehrmals längere Forschungsaufenthalte und damit auch intensive Recherchen im umfangreichen, noch nicht digitalisierten historischen Bestand des Instituts ermöglichte. 
des 17. Jahrhunderts erstmals erschien. In deren Tradition einer umfassenden Vermittlung der Realordnung der Dinge, die auch Arbeitssektoren und Arbeitsteilung miteinschließt, stehen die im 18. und frühen 19. Jahrhundert entstandenen Lehr- und Lesebücher eines Johann Heinrich Pestalozzi, Friedrich Fröbel, Joachim Heinrich Campe oder Johann Bernhard Basedow (vgl. Dietrichs 2010/1978, 48f.) - wenn auch mitunter einige der genannten Autoren im Vergleich zu Comenius durch die säkulare Idee der Aufklärung geprägt wurden und ihre Bemühungen stärker mit anderen weltanschaulichen Zielsetzungen verbanden als jenen eschatologischen, die für Comenius noch von Bedeutung waren. Die Spannung zwischen den an einer göttlichen Offenbarung orientierten Realienbüchern und jenen, die eine säkulareenzyklopädische Perspektive unter den Idealen der Aufklärung aufgriffen wie es insbesondere bei Basedow der Fall ist (vgl. Overhoff 2002, 166) prägte die Produktion der Realienbücher im 19. Jahrhundert.

Vor dem Hintergrund einer kurzen Skizze des Expansions- und Differenzierungsprozesses von Schulsystem und Schulbuchproduktion im 19. Jahrhundert (2.) und einer begriffsgeschichtlichen Erläuterung des Gewerbebegriffs (3.) werden im Beitrag folgende drei Perspektiven diskutiert: Zunächst wird dargelegt (4.), wie der Begriff des Standes mit dem Gewerbe verhandelt wird und wie damit bestimmte Kontinuitäten vorrevolutionärer, feudalstruktureller Gesellschaft verbunden blieben. Darauf hin (5.) wird der Blick auf das Verhältnis von Industrie und Handwerk gewendet. Vor dem Fazit (7.) wird der Blick auf die historische Metaphernanalyse und dessen Relevanz für die historische Berufsbildungsforschung im Zusammenhang der Schulbuchanalyse (6.) gelenkt.

\section{Schul- und Schulbuchentwicklung im 19. Jahrhundert}

Mit Übergang ins 19. Jahrhundert zeichnete sich die im 18. Jahrhundert bereits debattierte und teils legislativ initiierte Institutionalisierung eines Schulwesens in systemischer Hinsicht immer deutlicher ab. Müller beschreibt die Entwicklungsphase der systemischen Schulbildung in der ersten Hälfte des 19. Jahrhunderts als Phase der Systemfindung. Diese zeichnete sich vor allem durch Kontroversen über das richtige Schulsystem aus. Gegen Mitte des 19. Jahrhunderts hatte diese Phase der Kontroversen sich zugunsten eines bestimmten Systems konsolidiert. Müller bezeichnet diese Phase als Systemkonstituierung. Grob skizziert zeichnete sich dieses System durch höhere und niedere Schulen aus, die berechtigend aufeinander und im Bezug zur gesellschaftlichen Karrierewegen abgestimmt waren (vgl. Müller \& Zymek 1987, 35f.). Auch für die berufliche Bildung lässt sich ein Institutionalisierungsprozess fürs 19. Jahrhundert in systemischer Hinsicht beschreiben. Dies führte 
dazu, dass die Gewerbe- bzw. die Berufsschule sich zunehmend etablierte und das autoritäre Hausrecht des Meisters in Fragen der Ausbildung seiner Lehrlinge sukzessive eine Begrenzung erfuhr und unter staatliche Aufsicht gestellt wurde (vgl. Pätzold \& Wahle 2009, 31f.). Die theoretische Ausbildung gewann für die berufliche Bildung immer mehr an Bedeutung. Auch das berufsbildende Schulwesen expandierte und differenzierte sich im Verlauf des 19. Jahrhunderts (z.B. Harney \& Tenorth 1986).

Parallel zur Entstehung, Expansion und Differenzierung der Schulen und Schultypen des Bildungssystems setzte im ausgehenden 18. Jahrhundert die Produktion von Schulbüchern ein. Entsprechend der verzögerten Ausbaudynamik der beruflichen Schulen erfolgte auch die Produktion von Schulbüchern, die eigens für die beruflichen Schulen verfasst und im Titel auch so ausgewiesen wurden, erst zum letzten Drittel des 19. Jahrhunderts. Da es berufliche Fachlehrer wie Fachliteratur in der ersten Hälfte des 19. Jahrhunderts kaum gab, kamen bis auf wenige Ausnahmen zu Beginn der Gewerbeschulentwicklung Volksschullehrer zum Einsatz, die den beruflichen Unterricht mit den Lehrmitteln der Volksschule vollzogen (vgl. Zimmermann 1996). Insofern müssen im Rahmen einer historischen Schulbuchanalyse für die beruflichen Schulen die Materialen der Volksschulen auch als Materialien von Gewerbeschulen gewertet werden.

Das für diesen Beitrag gesichtete Quellenmaterial des Georg-EckartInstituts spiegelt diese Schulbuchentwicklung wider. Die Sammlung des Instituts weist Realienbücher, die eigens für die gewerblichen Schulen verfasst sind, überwiegend - aber dann in zunehmender Anzahl - ab Mitte des 19. Jahrhunderts aus. Aufgrund des Umfangs des historischen Bestandes des Georg-Eckart-Instituts kann das Quellenmaterial als repräsentativ aufgefasst werden.

\section{Gewerbebegriff und sozial-politische Semantik}

Der Begriff der Branchen war - anders als das heute der Fall ist - im 19. Jahrhundert zwar nicht unbekannt, wie beispielsweise Tagebucheintragungen von Unternehmern des frühen 19. Jahrhunderts belegen (z.B. Eberhart II zit. n. Mönnich 1971, 65), aber in der allgemein verbreiteten und verwendeten Sozial- und Wirtschaftssprache fand er keine Verwendung. Anders dagegen verhält es sich mit dem Begriff des Gewerbes, der dem heute geläufigeren Begriff der Branchen verwandt ist. Dieser Begriff prägte das sozial-politische Sprechen in Deutschland des 19. Jahrhunderts deutlich mit. Aus begriffsgeschichtlicher Sicht wird dieser aber nicht zu den kraftvollen historischen Grundbegriffen des sozial-politischen Sprechens im Deutschland des 19. Jahrhunderts gerechnet und mit dem Begriff Industrie diskutiert (vgl. Höl- 
scher 1982). Der Begriff des Gewerbes erfüllt für sich - um zu einem Grundbegriff des sozialpolitischen Sprechens zu werden - nicht das Grundbegriffe auszeichnende Kriterium der Unaustauschbarkeit, was ihn zum permanent strittigen, aber eben nicht aufgebaren Begriff erhebt (vgl. Koselleck 2010, 68), wie das beispielsweise für den Begriff der Arbeit gilt (vgl. Conze 1972). Die begriffsgeschichtliche Einordnung als Sekundarbegriff belegt auch die heute dem Gewerbebegriff fehlende gesellschaftspolitische Strahlkraft.

Der begriffsgeschichtliche Stellenwert des Gewerbebegriff, der durch die Industrialisierung beflügelt wird und phasenweise synonym mit Industrie war (vgl. Hölscher 1982, 278), erstreckt sich vor allem darin, dass der Begriff des Gewerbes ein wichtiger Rahmengebungsbegriff ist, aus dem sich in Kombination mit geschichtlichen Grund- oder anderen Rahmenbegriffen die Ordnungs-, Erfahrungs- und Erwartungshorizonte ergeben, die in der Semantik des Begriffs enthalten sind und die die soziale Wahrnehmung einer Epoche prägen. Insbesondere in Verbindung mit dem Begriff des Zweigs war im 19. Jahrhundert der Begriff des Gewerbes im Sinne des heutigen Branchenbegriffs virulent. Vorerst noch ungeachtet der im Begriff des „Gewerbezweigs“ enthaltenden Metaphorik wurde damit ein bestimmter Handels- oder Handwerksbereich definiert, ohne dass er an dessen Rändern besonders klar definiert wurde. Dem Begriff des Gewerbes bzw. dem der Branchen ist damit Bestimmt-Unbestimmtes angehängt, was dessen Anpassung an den Wandel der Arbeitswelt ermöglicht. Auch der Doppelbegriff , Gewerbefreiheit' steht in diesem Sinn und war gegen den Zunftzwang gerichtet (vgl. Conze 1972, 173). Derartige begriffliche Unbestimmtheit ermöglicht die Wiederholung von Strukturen im zeitlichen Verlauf, die zur Kontinuität von historischen Strukturmustern beitragen.

\section{Gewerbe zwischen Stand und Klasse, Bewahren und Erneuern}

Der epochemachende Übergang in die Moderne zeichnete sich vor allem durch die Entstehung einer von der Ständeordnung gelösten Gesellschaft aus, deren Positionen in der staatlichen Verwaltung fortan durch Leistung und nicht durch Erbe zu erreichen waren. Den Bildungsinstitutionen kam vor diesem Hintergrund eine wichtige Funktion zu, nämlich die der auf Leistung bezogenen Auslese. ,Klasse' wurde zu Beginn des 19. Jahrhunderts der sozial-politische Gegenbegriff zu Stand. Ihre Gegensätzlichkeit prägte die sozialpolitischen Auseinandersetzungen und Debatten des 19. Jahrhunderts (vgl. Koselleck 1989, 109). Gewerbe wurde im 18. Jahrhundert vor allem standespolitisch im Bezug zum Handwerk definiert. Gewerbefreiheit trat erst als 
gesellschaftliche Erneuerung der bürgerlichen Gesellschaft auf und wurde mit dem Klassenbegriff in Verbindung gebracht.

Nach Koselleck sind geschichtliche Zeitverläufe, auch wenn sie durch Ordnungsfundamente in hohem Maße scheinbar zerstörende Ereignisse wie zum Beispiel Revolutionen geprägt sind, nicht eben über alle Maße historische Strukturen verstörtend. Neben an Ereignissen erzählbaren Diskontinuitäten der Geschichte lassen sich in einem strukturgeschichtlichen Sinne auch historische Kontinuitäten in Form von sich wiederholende Strukturen beschreiben, die unberührt vom (Groß-)Ereignis auch nach dessen historischem Erscheinen weiterhin existent sind (vgl. Koselleck 2010b, 489). Insofern wurde die gesellschaftliche Entfaltung des neuen, sozial- und verwaltungspolitischen Ordnungsbegriff ,Klasse' am Anfang des 19. Jahrhunderts begleitet von gesellschaftlichen Strukturen, die dem alten Standesbegriff entstammten und die der neuen Ausrichtung des Gesellschaftsbewusstseins hemmend entgegenstanden. Es ist die These des nachstehenden analytischen $\mathrm{Ab}-$ schnitts, dass dieser Antagonismus auch in den Schulbüchern ablesbar ist und insbesondere im thematischen Zusammenhang des Verhältnisses von Arbeit, Gewerbe und Gesellschaft steht:

Jeder Stand hat besondere Rechte, damit ein Jeder desto leichter die Beschäftigungen verrichten kann, die seinem Stande zukommen, und kein Stand dem anderen hinderlich werde [GEI-Sig.: RCH-I 7(203,1853), S. 221].

Im Zitat ist der Standesbegriff rechtspolitisch verfasst. Der Klassenbegriff als der neue gegenfeudale Kampfbegriff hatte sich in der in Schwung kommenden Produktion der Realienbücher in der ersten Hälfte des 19. Jahrhunderts noch nicht vollends durchgesetzt. Dafür erfreute sich der Begriff des Standes bis Mitte des 19. Jahrhunderts weiterhin großer Beliebtheit unter den Verfassern der Lesebücher: „Bei uns Deutschen gibt es vier Stände, nämlich Fürsten, Edelleute, Bürger und Bauern“, heißt es beispielsweise in dem bereits zitierten Realienbuch, das kurz nach der gescheiterten bürgerlichen Revolution in Deutschland von 1848 bereits in der 203. Auflage erschien. Von Meritokratie und Gewerbefreiheit keine Rede: Die Stände werden gar noch als „erblich“ vorstellig, „d.h., die Kinder erben den Stand des Vaters“ [GEI-Sig.: Ebd., 220]. Das Beispiel zeigt exemplarisch wie präsent die vorrevolutionäre Gesellschaftsordnung im Bewusstsein der Verfasser von Realienbüchern scheinbar noch vorhanden war.

Auch wenn diese Vier-Stände-Einung der Gesellschaft in Realienbüchern, insbesondere der ersten Hälfte des 19. Jahrhunderts, weit verbreitet gewesen ist, so wurde nicht immer der Erbcharakter so dermaßen offen betont, wie in dem zitiert Beispiel. Gemein ist allen, dass die Stände-Einung vor allem in Bezug zur zu leistenden gesellschaftlichen Gesamtarbeit und der gesellschaftlichen Machtverteilung und -ausübung gebraucht wurde. So ist beispielsweise zu lesen: 
da die Obrigkeit mehr Gewalt haben müssen, als die übrigen Menschen, so müssen verschiedene Stände unter den Menschen entstehen; es müssen einige vornehm, andere gering, andere weder vornehm noch gering sein, oder zum Mittelstande gehören. Ein Mensch ist vornehmer, als der andere, das heißt so viel, als: ein Mensch hat mehr Ansehen und Macht, als ein anderer (...) doch sind die Bürger von den Bauern nur durch ihr Gewerbe und ihre Lebensart unterschieden, und nicht durch den Stand [GEI-Sig.: RCH-I 7(203,1853), 220].

Diese prestigedifferenzierte Vier-Stände-Einung erfuhr je nach Umfang der Zuwendung zum Thema eine Erweiterung, indem beispielsweise der „Gelehrte" und der "Soldat" als eigenständig zu betonender Stand hinzugenommen wurde [GEI-Sig.: RDH-I 12(1,1812), S. 562]. Dass letztere als eigene Stände bezeichnet und nicht einem, nämlich entsprechend der ständepolitischen Ordnung der vorrevolutionären Zeit dem Dritten Stand, zugeschlagen werden, zeigt, dass sich der in den Realienbüchern verwendete Begriff des Standes partiell jenem Begriffsverständnis geöffnet hatte, das mit dem Begriff der Funktionsklasse in Verbindung steht. Ferner ist auch auffällig, dass der Klerus in der Ständeaufzählung kaum noch Eingang fand. Die nachrevolutionäre Zersplitterung des in Revolutionszeit geeinigten Bürgertums wurde damit noch zementiert - ebenso wie das Faktum der Arbeitsteilung.

Dann noch Gott: Die Vier-Stände-Einung wurde in den Realienbüchern noch wie in der vorrevolutionären Zeit, insbesondere der ersten Hälfte des 19. Jahrhunderts, als eine von Gott gewollte Ordnung vorgestellt, die die herrschenden Klassen notfalls auch mit Gewalt zu verteidigen hätten:

Obrigkeit, welche die gute Ordnung mit Gewalt festhalten muß, weil viele Menschen ihr eigenes Wohl nicht beobachten wollen, erfüllt Gottes Wille. Ihr Zweck ist der Zweck Gottes, also ist von Gott gesetzt [GEI-Sig.: RDH-I 12(1,1812), 456].

Man geht nicht fehl, wenn man darin den Willen zu einer Untertanenpädagogik erkennt, die die Schul- und Erziehungspolitik des 19. Jahrhunderts prägte - auch und vor allem in der beruflichen Bildung (vgl. Leschinsky \& Roeder 1983, 344f.).

In diesem Zusammenhang stehen in den Realienbüchern immer wieder, den Leser mahnende Passagen, die daran erinnern, was der neue Mensch dieser ständischen Grundordnung zu verdanken und zu danken habe. Denn diese Ordnung riss den Menschen aus seinem elenden Naturzustand und brachte im Frieden und Zivilisation in einem damit ,wohleingerichteten Staate" [GEI-Sig.: RCH-I 7(203,1853), 210] - und das durch den Menschen selbst: des "elenden Lebens wurden die Menschen endlich überdrüssig, und die Vernünftigen unter ihnen besonders sannen darauf, wie diesem Unfug abzuhelfen sein möchte" [GEI-Sig.: Ebd.]. Als Garant für diese Ordnung wurden die bürgerlichen Gesetze vorstellig gemacht, oftmals in schlicht mit „Gesetze und Verordnungen“ überschriebenen Abschnitten. 
Die Gesellschaft ordnende Argumentation in Bezug auf Arbeitsteilung findet sich auch noch gegen Ende des 19. Jahrhunderts in nunmehr eigens für die beruflichen Schulen verfassten Realienbüchern. Zu bemerken ist, dass dies mit deutlich geringerem Gottbezug geschah, und mitunter, wie im folgenden Zitat, zwischen den Ständen im arbeitsfunktionalen Sinne eine metaphorisch begründete Enthierarchisierung einging, so wie es den innerständischen Bünden als Friedenssicherungsstrategie seit dem Mittelalter inne war (vgl. Koselleck 1994, 585):

Ein Staat ist ein strenggeordnetes, gutgeliedertes Ganzes, gleichsam ein Körper, bei dem jedes Glied seine Rechte und seine Pflichten hat. (...) Zwischen allen Gliedern muß ein unzerreißbarer Zusammenhang bestehen, und kein Glied soll sich über das andere erheben, den jedes ist notwendig, also der Bauer so notwendig als der Handwerker, oder der Gelehrte und Künstler, oder der Arzt und Richter, oder Kaufmann und Fabrikant, oder Pfarrer und Lehrer [GEI-Sig.: RF-II 12(2,1897), 317]

Die Unterschiede in Besitz und sozialem Ansehen zwischen den Berufsgruppen und Gewerben wurden nicht mehr intensiv thematisiert, wie das noch für die erste Hälfte des 19. Jahrhunderts zu konstatieren ist. Das ist kein Hinweis auf eine Nivellierung der Prestigegegensätze, sondern resultiert daraus, dass die Distinktion der sozialen Distanzen nach einem Jahrhundert Bürgergesellschaft Alltag und damit Habitus war. Eine besondere Erläuterung der bürgerlichen Sozialordnung in legitimierender Absicht bedurfte es nicht mehr auch Gott musste nicht mehr als schützende Hand zitiert werden. Legitimation erfuhr die Ordnung über die Arbeitsteilung, die aus dem Zusammenspiel der Klassen entstand, bei Störung gefährdet ist und damit zugleich das Staatsganze mit sich in den Untergang riss. Wo einst Gott für den Schrecken sorgen musste, sorgte nun mehr die Disfunktionalität der Arbeitsteilung dafür.

\section{Wandel der Arbeit: Verhältnis von Handwerk und Industrie}

Wenn zunächst seit Mitte des 19. Jahrhunderts nur langsam, so aber doch unaufhaltsam entfaltete sich zum Übergang ins 20. Jahrhundert der durch die zunehmende Industrialisierung evozierte Strukturwandel der Sozialschichten. Immer weniger Menschen wurden in der Landwirtschaft beschäftigt und der alten Mittelstand von Kleingewerbetreibende, Handwerkern und Bauern reduzierten sich zugunsten eines neuen, sich vor allem durch die Industriearbeit definierenden Mittelstandes (vgl. Kap. 2 in Kocka 1981). Mit diesem gesellschaftlichen Wandel ging ein Wandel des Bewusstseins von Arbeit, Bildung und Konsum einher. Auch die Schulbücher reagierten auf diese 
Entwicklung und halfen mit, den neuen Mittelstand und den damit verbundenen Funktionswandel des alten Mittelstandes gesellschaftlich zu etablieren. Im sozialpolitischen Sprechen schlug sich diese Spaltung in den Begriffen „Kleingewerbe“ und „Großgewerbe“ nieder, also Handwerkstatt versus Fabrik. Mit diesem Antagonismus steht nicht gleich der völlige Abgesang des Handwerks oder das vollmundige Lob der Fabrik in Verbindung. Nein, auch das Handwerk bekam in den Schulbüchern noch Wert zugesprochen, wenngleich nicht ohne despektierliche Rüge: Betonung fand zum Bespiel dessen Rückständigkeit in beruflichen Arbeitsfeldern wie zum Beispiel dem der Buchhaltung:

Die Menge solcher Geschäftsvorgänge läßt sich nicht im Kopfe behalten, und auch die Schiefertafel, welche bislang vielfach die verschiedenen Geschäftsbücher vertrat, genügt da nicht mehr [GEI-Sig.: RF-II 1(22,01)-1, 219].

Das Handwerk wurde also trotz dessen Rückständigkeit und der scheinbaren Vormachtstellung der Industrie aber weiterhin als funktional vorgestellt. Stolz und Ehre werde es nicht gleich verlieren. „Allerdings“, so heißt es unter diesen Leitbegriffen in einem Realienbuch aus dem Jahr 1887,

ist es richtig, daß z.B. der Flaschner die Lampen besser und billiger in der Fabrik kauft, als er sie selbst herzustellen vermag. Daran thut er klug, sich bei diesem und manchen anderen Artikel auf den Handel und die Vornahme von Ausbesserungen zu beschränken [GEI-Sig.: RF-II 12(2,1897), 74].

Der Funktionswandel tat dem Gedanken der Arbeitsteilung in der Vorstellung des großen Kreislaufs der Produktion aber keinen Abbruch:

Jeder muss seine besondere Arbeit in die große allgemeine Arbeit des Volkes einreihen können [GEI-Sig.: RF-II 1(22,01)-1, 218].

Gibt sich hier die konnotierte Verbindung von Arbeit, Gewerbe und Volk zu erkennen, auf die später auch die Nationalsozialisten bauen konnten, so finden sich in anderen Passagen zudem noch die rügende Betonung der jedem Beruf eigenen Begrenzung:

Jeder Beruf hat seinen angestammten Scharfblick und seine angestammte Borniertheit [GEI-Sig.: DF-I 1(1,1862), 615].

Diese Passage ist Lob und Tadel zugleich: Gelobt wird das Fachliche, was einen Beruf ausmacht, getadelt wird eine habituelle Begrenztheit der Berufe. Letzteres wohl vor allem auch, da Borniertheit der Anpassung von Beru-fen im Rahmen von Wandel im Wege steht. Anpassen sollte sich aber nicht nur das Handwerk an die Rollenzuweisung im Zuge der zunehmenden industriellen Produktion von Waren, sondern vor allem auch die Industrie, die sich ja stets den modernen Produktionsbedingungen anpassen musste und diese in die Ausbildung des Arbeiternachwuchses investieren sollten. Schülern wurde damit eine Vorstellung von industrieller Flexibilität vermittelt. 


\section{Exkurs: Metaphorologische Schulbuch- und Berufsbildungsforschung}

Glied und Körper, Zweig und Ganzes sowie Bilden und Bauen - um einige Begriffe zu nennen, die in der vorstehenden Diskussion in Erscheinung getreten sind - werden in der metaphorologischen Forschung als Titelmetaphern bezeichnet (vgl. Konersmann 2007). Anders als im Diskurs der allgemeinen Erziehungs- und Sozialwissenschaft (vgl. Ragutt \& Zumhof 2016) existiert keine metaphorologische Schulbuchforschung (vgl. Fuchs, Niehaus \& Stoletzki 2014, 21f.). Die historische Schulbuchforschung - unabhängig ob für die allgemeine oder die berufliche Bildung - betritt mit der Metaphorologie Neuland.

Der Unterschied zwischen der sprachwissenschaftlichen und philosophischen Metaphernanalyse besteht seit Hans Blumenberg darin, dass das Denken in Metaphern eine Form der Beherrschung der nicht zu überblickenden Wirklichkeit darstellt. Metaphern sind der primäre Wirklichkeitsbezug (vgl. Blumenberg 1976, 123). So verstandene Daseinsmetaphern entfalten eine epochenprägende und Verhalten induzierende Pragmatik (vgl. Blumenberg 1999, 25), die es hermeneutisch zu beschreiben gilt. Insbesondere in historischen Phasen mit enormen Umbrüchen, wie etwa dem der Aufklärung, ist der Bezug auf Metaphern im Sprechen über Welt nicht konsolidiert. Daseinsmetaphorisch herrscht eine hohe Instabilität und allerhand metaphorische Bezüglichkeiten werden als Angebot der Daseinsorientierung in den Diskurs gestellt (vgl. Blumenberg 1962). Diese wenig konsolidierte Situation des Geistes prägte vor allem das frühe 19. Jahrhundert entschieden mit. Vor dem Hintergrund der Metaphorologie im Allgemeinen wie der geistigen Situation des 19. Jahrhunderts im Besonderen steht die These, dass das Schulbuch keine Metaphernfreiheit für sich behaupten kann und im 19. Jahrhundert (wie wohl auch davor und danach) an der metaphorischen Arbeit der Beherrschung der Wirklichkeit maßgeblich miteinbezogen gewesen ist, um der „Welt und den in ihr waltenden Mächten die reine Willkür nicht überlassen“ (vgl. Blumenberg 2006, 50) zu müssen. Erst jüngst hatte Egbert Witte herausgearbeitet, wie Labyrinth und Fluidum als Metaphern in der (Bildungs-) Philosophie Comenius' eingegangen sind (vgl. Witte 2012) und stabilisierend auf die damalige Geistestätigkeit wirkten. Eine unbeantwortete Frage in diesem Zusammenhang ist nicht nur, wie die erwähnte Lesefibel Comenius' in dessen eigene Metaphernverwendung eingegangen ist, sondern wie die Schulbuchentwicklung des 19. Jahrhunderts davon berüht war.

Im Sinne der von Blumenberg analysierten „Lesbarkeit der Welt“ (1986) als Metapher für die Garantie auf weltliche Verfügbarkeit kann das schulische Lesebuch der Realien - also das Buch der realen Welt - par excellence selbst bereits als eine Metapher aufgefasst werden. Es bietet in seiner ganzen 
Form tröstende Gewissheit auf humane Selbstbehauptung im nicht zu übersehenden Ganzen, da es exemplarisch über alle Belange des irdischen wie himmlischen Lebens aufzuklären scheint. Eine umfänglich metaphorologische Forschung hätte hierbei allerdings zunächst einmal den Einzug sowie die Neuproduktion von Metaphorik im Epochenwandel zur Moderne im Schulbuch zu analysieren. Hierzu ein Beispiel: Unter dem Sichtwort der „Berufswahl“ führen die Verfasser eines Realienbuchs für berufliche Schulen im Jahre 1902 aus:

Jeder Beruf birgt große Tiefen und unbekannte Minen. Jeder Schlag der Hacke, jeder Tag der Arbeit öffnet uns verborgene Schätze. In jedem Berufe steigt man aus dem Nichts der vollkommenen Unfähigkeit durch die unendlichen Stufen der Mittelmäßigkeit zur Vollkommenheit auf (...). Ein verfehlter Beruf! Ein verfehltes Leben! [GEI-Sig.: RF - II 1(22,01)-1].

Das Zitat gibt in mehrfacher Hinsicht Daseinsmetaphorik zu erkennen, die im Anschluss an Blumenberg den Gang der europäischen Geistesgeschichte entschieden mitbestimmten. Zum ersten wäre zu nennen, die Metaphorik der terra incognita, die im Zitat mit Verweis auf die „großen Tiefen“" und die „unbekannten Minen“ mit dem Beruf und einer erwartungsvollen Zukünftigkeit (,verborgener Schatz') verbunden ist. Erst diese froh stimmende Formel der terra incognita macht die Mühen des ungewissen Lebens ertragbar. Die Höhlenmetaphorik, der Blumenberg sich ausgiebig in Höhlenausgänge zugewendet hatte und die für die paideia nicht unwesentlich ist (z.B. Brumlik 2016), wird im Zitat im Moment des Progresses vom „Nichts der vollkommenden Unfähigkeit“" zur „Vollkommenheit“ deutlich, die hier ohne Zweifel als Arbeit an Erkenntnis und Menschwerdung zu verstehen ist. Das Schulbuch (beziehungsweise dessen Verfasser) ist in die Rolle desjenigen versetzt, dem die Aufgabe zukommt, die Menschen in der platonischen Höhle von den Schattenbildner zu lösen und zu Erkenntnis zu bringen. Das Fazit der zitierten Passage, dass ein verfehlter Beruf ein verfehltes Leben erwirke, steht im Sinne einer Lebensmetaphorik, wie sie Blumenberg beispielsweise in der Philosophie Georg Simmels nachgegangen ist (vgl. Blumenberg 1976).

\section{Ausblick}

Eine systematische Historiographie der Realienbücher für die institutionalisierte Berufsbildung des 19. und auch 20. Jahrhunderts steht noch aus. Der vorliegende Beitrag konnte dieses Desiderat nur skizzen- und thesenhaft unter begriffsgeschichtlichen und metaphorologischen Gesichtspunkten erhellen. Angesichts der Skizze lässt sich folgender Ausblick auf die historische Schulbuchforschung für die berufliche Bildung werfen: 
(1) Eine wichtige, im vorliegenden Beitrag außer Acht gelassene Perspektive ist eine stärkere regionale und institutionelle Kontextualisierung der Realienbücher. Wer - gemeint sind Verlag sowie Verfasser - begann mit der Produktion von Unterrichtsbüchern für die sich sukzessive entwickelnden beruflichen Schulen? Welche Bücher erschienen wie oft, mit welcher Auflage und waren Vorbilder für andere Schulbuchproduktionen? Wie gehen die Weltanschauungskämpfe in die Schulbücher für Gewerbeschulen ein? Wann und wo entwickelte sich ein Korpus von Berufsbildungsbüchern?

(2) Neben dem Ausbau der metaphorologischen wie begriffsgeschichtlichen Forschung ist eine weitere, sicherlich fruchtbare Forschungsperspektive die, die aus den Theorien zur Neueren Politik- und Sozialgeschichte resultieren und die auch in der bildungshistorischen Forschung diskutiert wird. In der Neuren Politikgeschichte wird das Rituelle, das offen und verdeckte Zeremonielle, eben das sich in Traditionen eigentümlich wiederholende Strukturelle in den Blick genommen. Kontinuitäten und Diskontinuitäten politischen Handelns und politischen Bewusstseins sollen so erhellt werden wie auch dem hier zugrundeliegenden habituellen Denken (z.B. Fervert 2005; Zymek 2006). Für die Einordnung des Rituellen, des Zeremoniellen, des Mythischen usw. sind nicht nur die Semantiken begriffsgeschichtlich zu klären, sondern auch deren metaphorologische Hintergründe.

(3) Lange diachrone, quellennahe Betrachtung der historischen Schulbuchforschung sind zeiträumlich kurz gefassten vorzuziehen. Epochale Perspektiven sind damit nicht ausgeschlossen. Denn die Veränderungsprozesse in Schulbüchern sind, wenn auch kontinuierlich, so aber doch zäher und vor allem doch durch gesellschaftliche Epochenbrüche charakterisiert (vgl. Jacobmeyer 2011, 9f.). Diachrone Studien gegeben den Strukturwandlungsprozess in Medien wie dem Schulbuch besser wieder und erlauben eine schärfere Wirkungsbeurteilung historischer Ereignisse und chronologischer Verläufe.

(4) Und schließlich: Es empfehlt sich die Analyse im Bezug auf Themenfelder vorzunehmen. Diese könnten z.B. das Meister-Lehrling-Verhältnis, also Machtbeziehungen, die Beziehung zwischen Staat und Lehre, Lebensund Arbeitsverhältnisse des Proletariats usw. sein.

\section{Literatur}

Blumenberg, H. (2006). Arbeit am Mythos. Frankfurt am Main: Suhrkamp.

Blumenberg, H. (1999). Paradigmen zu einer Metaphorologie. Frankfurt am Main: Suhrkamp.

Blumenberg, H. (1986). Die Lesbarkeit der Welt. Frankfurt am Main: Suhrkamp. 
Blumenberg, H. (1976). Geld oder Leben. Eine metaphorische Studie zur Konsistenz der Philosphie Georg Simmels. In H. Böhringer \& K. Gründer (Hrsg.), Ästhetik und Soziologie um die Jahrhundertwende: Georg Simmel (S. 121-134). Frankfurt am Main: Klostermann.

Blumenberg, H. (1962). Ordnungsschwund und Selbstbehauptung. Über Weltverstehen und Weltverhalten im Werden der technischen Epoche. In H. Kuhn \& F. Wiedmann (Hrsg.), Das Problem der Ordnung. VI. Deutscher Kongress für Philosophie. München 1960 (S. 37-57). Meisenhein am Glan: Hain.

Brumlik, M. (2016). Ausgänge aus dem Höhlengleichnis? Hans Blumenberg gegenüber einem, nein: dem klassischen pädagogischen Bild. In F. Ragutt \& T. Zumhof (Hrsg.), Hans Blumenberg: Pädagogische Lektüren (S. 21-34). Weinheim: Springer.

Conze, W. (1972). Arbeit. In O. Brunner, W. Conze \& R. Koselleck (Hrsg.), Geschichtliche Grundbegriffe. Historisches Lexikon zur politisch-sozialen Sprache in Deutschland, Bd. 1 (S. 154-215). Stuttgart: Klett.

Dietrichs, U. (2010/1978). Annäherung an das Schulbuch. Geschichte und Definition eines umstrittenen Begriffs. Berlin, Hildesheim: o.V. Online: http://www2.huberlin.de/sachbuchforschung/MEDIA/abfdsbf/Arbeitsblaetter_Sachbuchforschung_18.pdf(24.11.2015).

Durovic, A. \& Matthes, E. (Hrsg.). (2010). Freund-und Feindbilder in Schulbüchern. Bad Heilbrunn: Klinkhardt.

Fervert, U. (2005). Neure Politikgeschichte: Konzepte und Herausforderungen. In U. Frevert \& H.-G. Haupt (Hrsg.), Neue Politikgeschichte. Perspektiven einer historischen Politikforschung, (S. 7-26). Frankfurt am Main: Campus.

Fuchs, E., Nienhaus, I. \& Stoletzki, A. (2014). Das Schulbuch in der Forschung. Analysen und Empfehlungen für die Bildungspraxis. Göttingen: V\&R Unipress

Harney, K. \& Tenorth, H.-E. (1986). Berufsbildung und industrielles Ausbildungsverhältnis. Zur Genese, Formalisierung und Pädagogisierung beruflicher Ausbildung in Preußen bis 1914. Zeitschrift für Pädagogik, 32(1), 9-14.

Heinze, C. (2013). Nützliches Wissen, Untertanengeist und Gottesfurcht. Zur Konstruktion von Kindheit in preußischen Volksschullesebüchern. In: M. Winzen (Hrsg.), Kindheit. Eine Erfindung des 19. Jahrhunderts (S. 173-194). Oberhausen: Athena.

Heinze, C. (2010) (Hrsg.). Das Bild im Schulbuch. Bad Heilbrunn: Klinkardt.

Hölscher, L. (1982). Industrie, Gewerbe. In O. Brunner, W. Conze \& R. Koselleck (Hrsg.), Geschichtliche Grundbegriffe. Historisches Lexikon zur politischsozialen Sprache in Deutschland. Band 3 (S. 237-304). Stuttgart: Klett.

Jacobmeyer, W. (2011). Das deutsche Schulgeschichtsbuch, 1700-1945. Die erste Epoche seiner Gattungsgeschichte im Spiegel der Vorworte. Berlin: LIT.

Kocka, J. (1981). Die Angestellten in der deutschen Geschichte. Göttingen: Vandenhoeck\&Ruprecht.

Konersmann, R. (Hrsg.). (2007). Wörterbuch der philosophischen Metaphern. Darmstadt: Wiss. Buchges.

Koselleck, R. (2010). Die Geschichte der Begriffe und die Begriffe der Geschichte. In R. Koselleck (Hrsg.), Begriffsgeschichten (S. 56-76). Frankfurt am Main: Suhrkamp. 
Koselleck, R. (2010b). Diesseits des Nationalstaates. Föderale Strukturen der deutschen Geschichte. In: R. Koselleck (Hrsg.), Begriffsgeschichten (S. 486-503). Frankfurt am Main: Suhrkamp.

Koselleck, R. (1994). Bundnis, Föderalismus, Bundestaat. In: O. Brunner, W. Conze \& R. Koselleck (Hrsg.), Geschichtliche Grundbegriffe. Historisches Lexikon zur politisch-sozialen Sprache in Deutschland. Bd. 1 (S. 582-671). Stuttgart: Klett.

Koselleck, R. (1989). Begriffsgeschichte und Sozialgeschichte. In: R. Koselleck (Hrsg.), Vergangene Zukunft. Zur Semantik geschichtlicher Zeiten (S. 107-129). Frankfurt am Main: Suhrkamp.

Leschinsky, A. \& Roeder, P. M. (1983). Schule im historischen Prozeß. Zum Wechselverhältnis institutioneller Erziehung und gesellschaftlicher Entwicklung. Stuttgart: Klett-Cotta.

Matthes, E. \& Heinze, C. (Hrsg.). (2006). Die Familie im Schulbuch. Bad Heilbrunn: Klinkardt.

Mönnich, H. (1971). Aufbruch ins Revier - Aufbruch nach Europa 1871-1971. München: Bruckmann.

Müller, D. K. \& Zymek, B. (1987). Sozialgeschichte und Statistik des Schulsystems in den Staaten des Deutschen Reiches 1800-1945. Datenhandbuch zur deutschen Bildungsgeschichte. Band II. Höhere und mittlere Schulen. Teil 1. Göttingen: Vandenhoeck\&Ruprecht.

Overhoff, J. (2002). Johann Bernhard Basedow als Hauslehrer auf Borghorst. Sein Erziehungsprogramm von 1752 im Lichte neuer Quellenfunde. In Sektion Historische Bildungsforschung in der DGfE (Hrsg.), Jahrbuch Historische Bildungsforschung (Bd. 8, S. 159-180). Bad Heilbrunn: Klinkardt.

Pätzold, G. \& Wahle, M. (2009). Ideen- und Sozialgeschichte der beruflichen Bildung. Entwicklungslinien der Berufsbildung von der Ständegesellschaft bis zur Gegenwart. Baltmannsweiler: Schneider.

Ragutt, F. \& Zumhof, T. (2016). Die Lesbarkeit des Werks. Einleitende Bemerkungen zur pädagogischen Lektüre von Hans Blumenberg. In F. Ragutt \& T. Zumhof (Hrsg.), Hans Blumenberg: Pädagogische Lektüren (S. 21-34). Weinheim: Springer.

Siegfried, K.-J. (1979). Zur politischen Funktion des Schulbuchs im Faschismus. Demokratische Erziehung, 5(1), 44-51.

Tröhler, D. \& Oelkers, J. (2005). Historische Schulbuchforschung und Steuerung des Schulsystems. In E. Matthes \& C. Heinze (Hrsg.), Das Schulbuch zwischen Lehrplan und Unterrichtspraxis (S. 95-107). Bad Heilbrunn: Klinkardt.

Waiter, W. (Hrsg.). (2003). Schulbuchforschung in Europa - Bestandsaufnahme und Zukunftsperspektive. Bad Heilbrunn: Klinkhardt.

Witte, E. (2012). Labyrinth und Fluidum. Metaphorologische Vorbemerkungen zum Bildungsgehalt der Dinge bei Comenius und Bacon. In A. Dörpinghaus \& A. Nießeler (Hrsg.), Dinge in der Welt der Bildung - Bildung in der Welt der Dinge (S. 29-56). Würzburg: Königshausen \& Neumann.

Zimmermann, C. (1996). „Blinde Anschauung soll sich zum klaren Begriff und zu fertiger Übung erheben“. Die Wertheimer Gewerbeschule 1836-1862. Wertheimer Jahrbuch, 195-226.

Zymek, B. (2006). Neuere Politikgeschichte und Sozialgeschichte des Bildungswesens. In G. Miller-Kipp \& B. Zymek (Hrsg.), Politik in der Bildungsgeschichte. Befunde, Prozesse, Diskurse (S. 35-52). Bad Heilbrunn: Klinkhardt. 


\section{Quellen:}

GEI-Sig.: DF-I 1 $(1,1862)$

Dresdner Lesebuch für Handelsschulen sowie für Real- und höhere Bürgerschulen. Hrsg. v. Dr A. Benser, S. Kluge. Erschienen bei August Schulz-Verlag in Leipzig im Jahre 1862

GEI-Sig.: RF-II 1(22,01)-1

Lehr- und Lesebuch für Fortbildungs-, Gewerbe- und Handelsschulen. Hrsg. v. Franz Schürmann, Friedrich Windmöller. 22. Auflage. Erschienen bei Bädeker in Essen, 1901

GEI-Sig.: RF-II12(2, 1897)

Lesebuch für Fortbildungsschulen. Hrsg. v. Württembergischen Evangelischen Schullehrerunterstüztungsverein. 2. Auflage. Erschienen bei Bonz in Stuttgart, 1897

GEI-Sig.: RCH-I 7(203, 1853)

Der deutsche Kinderfreund, ein Lesebuch für die Volksschule. Hrsg. v. F. D. Wilmsen. Als 203. Auflage erschienen bei G. Reimer in Berlin, 1853

GEI-Sig.: RDH-I 12(1, 1812)

Allgemeines Lesebuch, für Bürger und Landmann, vornehmlich zum Gebrauch in Stadt- und Landschulen. Erste Auflage. Erschienen bei Goethardt'sche Buchhandlungen in Bamberg und Würzburg, 1812 [Online-Ausg.: urn:nbn:de:0220gd-11209165]

Lesebuch für Preußische Schulen. Teil 1. Erschienen im Verlag Ferdinand Riegel in Potsdam, 1943 


\section{Herausgeberschaft}

Prof. Dr. Susan Seeber

Georg-August-Universität Göttingen

Professur für Wirtschaftspädagogik und Personalentwicklung

Susan.seeber@wiwi.uni-goettingen.de

Prof. Dr. Jürgen Seifried

Universität Mannheim

Lehrstuhl für Wirtschaftspädagogik - Berufliches Lehren und Lernen

seifried@bwl.uni-mannheim.de

Prof. Dr. Birgit Ziegler

Technische Universität Darmstadt

Institut für Allgemeine Pädagogik und Berufspädagogik

ziegler@bpaed.tu-darmstadt.de

\section{Autorinnen und Autoren}

Prof. Dr. Alexandra Bach (geb. Eder)

Universität Kassel

Institut für Berufsbildung

alexandra.eder@uni-kassel.de

Christian Dittmann, M.A.

Leibniz Universität Hannover

Institut für Berufspädagogik und Erwachsenenbildung

christian.dittmann@ifbe.uni-hannover.de

Prof. Dr. Rolf Dobischat

Universität Duisburg Essen

Fachgebiet Wirtschaftspädagogik / Berufliche Aus- und Weiterbildung rolf.dobischat@uni-due.de

Prof. Alison Fuller, PhD

UCL Institute of Education

University College London

alison.fuller@ucl.ac.uk 
Prof. Dr. Karl-Heinz Gerholz

Universität Bamberg

Professur für Wirtschaftspädagogik

Karl-Heinz.Gerholz@uni-bamberg.de

Prof. Dr. Philipp Gonon

Universität Zürich

Institut für Erziehungswissenschaft - Lehrstuhl für Berufsbildung

gonon@ife.uzh.ch

Dr. Julia K. Gronewold

Leibniz Universität Hannover

Institut für Berufspädagogik und Erwachsenenbildung

julia.gronewold@ifbe.uni-hannover.de

Ass.-Prof. Mag. Dr. Christoph Helm

Johannes Kepler Universität Linz

Abteilung für Pädagogik und Pädagogische Psychologie

christoph.helm@jku.at

Lena Hillebrecht, M.Ed.

Georg-August-Universität Göttingen

Professur für Wirtschaftspädagogik und Personalentwicklung

lena.hillebrecht@wiwi.uni-goettingen.de

Heike Jost, M.Sc.

heike.jost09@gmail.com

lic. phil. Stefan Kessler

Universität Zürich

Institut für Erziehungswissenschaft - Lehrstuhl für Berufsbildung

stefan.kessler@ife.uzh.ch

Lorenz Lassnigg

Institut für Höhere Studien (IHS) Wien

lassnigg@ihs.ac.at

Mag. Stefanie Antonia Mayer

Johannes Kepler Universität Linz

Absolventin der Wirtschaftspädagogik

mayer_steffi_91@yahoo.de 
Dr. Andreas Rausch

Otto-Friedrich-Universität Bamberg

Lehrstuhl für Wirtschaftspädagogik

andreas.rausch@uni-bamberg.de

Dr. Frank Ragutt

Europa-Universität Flensburg

Institut für schleswig-holsteinische Zeit- und Regionalgeschichte (IZRG)

ragutt@irzg.de

Andy Schäfer, Master of Arts

Universität Duisburg Essen

Fachgebiet Wirtschaftspädagogik / Berufliche Aus- und Weiterbildung andy.schäfer@uni-due.de

Dr. Juliana Schlicht

Universität Leipzig

Institut für Wirtschaftspädagogik

schlicht@uni-leipzig.de

Dr. Christian Schmidt, Professor i. V.

Universität Kassel

Fachgebiet Berufs- und Wirtschaftspädagogik

christian.schmidt@uni-kassel.de

Philipp Struck, M.A.

Leibniz Universität Hannover

Institut für Berufspädagogik und Erwachsenenbildung

philipp.struck@ifbe.uni-hannover.de

Prof. Dr. Manfred Wahle

Universität Duisburg Essen

Fachgebiet Wirtschaftspädagogik / Berufliche Aus- und Weiterbildung manfred.wahle@uni-due.de

Prof. Dr. Ursula Walkenhorst

Universität Osnabrück

Lehrstuhl für Didaktik der Gesundheits- / Humandienstleistungsberufe

ursula.walkenhorst@uni-osnabrueck.de 
Dr. Marcel Walter

Universität Duisburg Essen

Fachgebiet Berufspädagogik / Berufsbildungsforschung

marcel.walter@uni-due.de

Dr. Julia Warwas

Otto-Friedrich-Universität Bamberg

Lehrstuhl für Wirtschaftspädagogik

julia.warwas@uni-bamberg.de

lic. phil. Lea Zehnder

Universität Zürich

Institut für Erziehungswissenschaft - Lehrstuhl für Berufsbildung

lea.zehnder@ife.uzh.ch 